\title{
Physiological and behavioral responses, and their variability, in squid, Doryteuthis pealeii, embryos and paralarvae reared under chronic ocean acidification
}

\author{
by \\ Casey James Zakroff \\ M.Sc., Marine Science, King Abdullah University of Science and Technology, 2013 \\ B.S., Biology, Florida Gulf Coast University, 2012 \\ B.A., Anthropology, Florida Gulf Coast University, 2012 \\ Submitted in partial fulfillment of the requirements for the degree of \\ Doctor of Philosophy \\ at the \\ MASSACHUSETTS INSTITUTE OF TECHNOLOGY \\ and the \\ WOODS HOLE OCEANOGRAPHIC INSTITUTION
}

September 2019

(C) 2019 Casey James Zakroff. All rights reserved.

The author hereby grants to MIT and WHOI permission to reproduce and to distribute publicly paper and electronic copies of this thesis document in whole or in part in any medium now known or hereafter created.

Signature of Author

Joint Program in Oceanography/Applied Ocean Science and Engineering Massachusetts Institute of Technology and Woods Hole Oceanographic Institution July 30, 2019

Certified by

Dr. T. Aran Mooney Associate Scientist

Woods Hole Oceanographic Institution Thesis Supervisor

Accepted by

Dr. Mick Follows

Chair, Joint Committee for Biological Oceanography Massachusetts Institute of Technology 


\title{
Physiological and behavioral responses, and their variability, in squid, Doryteuthis pealeii, embryos and paralarvae reared under chronic ocean acidification
}

\author{
by \\ Casey James Zakroff \\ Submitted to the MIT-WHOI Joint Program in Oceanography/Applied Ocean Science and \\ Engineering on July 30, 2019 in partial fulfillment of the requirements for the degree of Doctor \\ of Philosophy in Biological Oceanography.
}

\begin{abstract}
Ocean acidification (OA) and related stressors, like warming, are occurring rapidly in coastal systems. There is concern about the impacts these stressors may have on the early development of species that use the nearshore as nursery habitat. The inshore longfin squid, Doryteuthis pealeii, plays an important role in the northwest Atlantic food web, and annually lays its eggs in the nearshore benthos during summer. This thesis sought to characterize morphological, physiological, and behavioral responses of $D$. pealeii embryos and paralarvae to OA. Experiments began in 2013, where I exposed squid eggs to a range of acidification levels (400 $2200 \mathrm{ppm} \mathrm{CO}_{2}$ ) to uncover when the dosage impacts first appear (around $1300 \mathrm{ppm}$ ). To do this, I developed multiple methods to better characterize the morphological changes and surface degradation of statoliths due to acidification. This initial work demonstrated small-scale variability in response intensity, across hatching days and the breeding season. I ran swimming behavior experiments with subsampled paralarvae from 2013 - 2015 and developed a novel 3D recording and analysis tracking system in the process. The 2D data from 2013 showed significant decreases in time spent near surface, while 3D data in subsequent years showed slight impacts to activity and swimming velocity with increasing acidification. Overall, I ran experiments from 2013-2016, and compiled and compared these data using response ratios. I show that seasonal temperatures impact the baseline state of the paralarvae through parental condition, while acidification sensitivity appears driven by parental year class. Finally, I examined the interaction of acidification stress with warming, demonstrating an antagonistic relationship between these stressors for this life stage of this squid. These data indicate that acidification builds as a stressor, impacting late stages of embryonic development, while warming impacts embryos early in development, and likely reduces acidification impacts by decreasing development time. This dissertation demonstrates that while the embryonic and paralarval stages can be sensitive to acidification, being so highly fecund and varying in resistance at multiple temporal scales allows for a substantial potential for resilience to a changing ocean in this population of squid.
\end{abstract}

Thesis Supervisor: Dr. T. Aran Mooney

Title: Associate Scientist with Tenure, Woods Hole Oceanographic Institution 
GRAPHICAL ABSTRACT

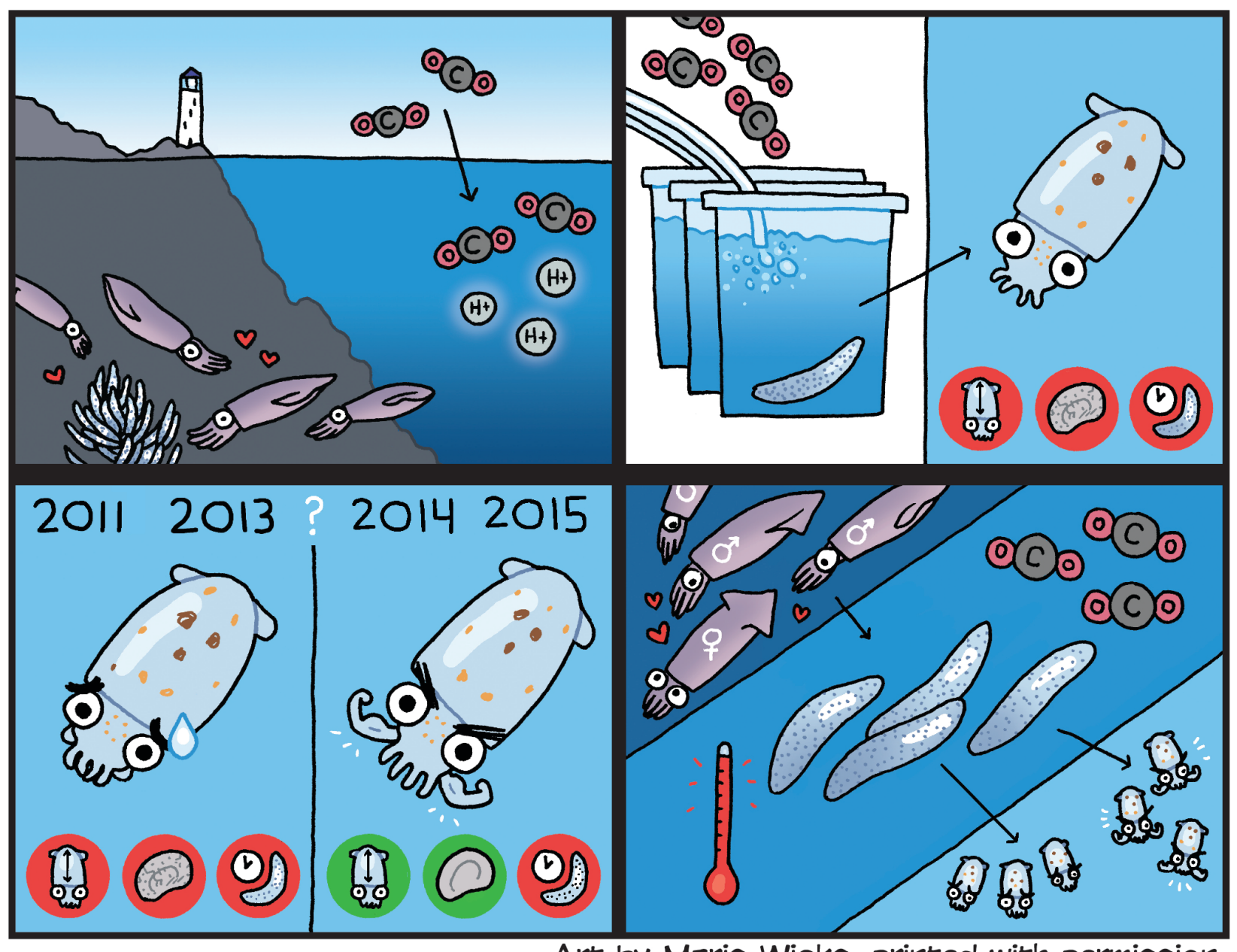

Art by Maris Wicks, printed with permission. 


\section{ACKNOWLEDGMENTS}

The National Science Foundation provided funding for this project under Grant No. 1220034 to Aran Mooney. The Woods Hole Oceanographic Institution also funded research presented in this dissertation through the Coastal Ocean Institute and Grassle Family Foundation awards.

The National Science Foundation Graduate Research Fellowship provided tuition and stipend support under Grant No. 1122374. The Hugh Hampton Young Memorial Fund Fellowship provided through the MIT Office of Graduate Education also provided tuition and stipend support, as did the Academic Programs Office of the Woods Hole Oceanographic Institution.

The Mindlin Foundation, through their OneTweetOnePercent award, and the Academic Programs Office of the Woods Hole Oceanographic Institution provided support for time pursuing outreach and science communication projects.

I would like to begin by acknowledging the lands occupied during this dissertation and the indigenous nation, the Mashpee Wampanoag, from whom they were taken. Please engage with and support the Wampanoag efforts to maintain and solidify their sovereignty, particularly if you are a resident of these lands as well. Otherwise a simple search online will provide you the knowledge of whose lands you currently occupy (in the Americas, at least) and which indigenous nations you could support as an ally through practices of being good kin.

I take this moment of writing as an opportunity for reflection and to earnestly acknowledge all those that have gotten me to and through this moment in my life. A PhD dissertation is a long and arduous process even with substantive support, and so to those who find themselves struggling in the mire of it: reach out, ask for help, and find that support.

I would like to thank my advisor, Dr. T. Aran Mooney, for his advice, support, and patience over these many years of squid experiments, confounding results, and new project ideas. Thank you for giving me the platform and the space to try and fail and succeed at too many things. Thank you for the discussions of social change in academia and for the guidance in scientific and career pursuits. I felt like we shifted over the course of this $\mathrm{PhD}$ from a relationship of respect between advisor and student, to one between colleagues, and I appreciate that immensely.

Aran did a fantastic job of bringing in people to support this project and I am deeply grateful for all of the support, insight, and assistance these collaborators provided. It would not have been possible to complete this project in all of its forms without their aid. Foremost, I want to thank Andrea Schlunk, who between taking over for me on the project while I was finishing my Master's degree, being a constant friend and colleague to bounce ideas off of, and being the most excellent statolith microdissection specialist among us, was instrumental to the completion of my $\mathrm{PhD}$. Research assistant Colin Wirth was also an incredible help, particularly in the development and execution of the behavioral experiments. I was extremely lucky to have the benefit of mentoring excellent Summer Student Fellows, Mary Ann Lee and Doriane Weiler, MBL Biological Discovery in Woods Hole students, Apryle Panyi, Lucy Fitzgerald, and Toni Sleugh, 
guest students, Jessica Wingar, field interns, Caitlyn Bozza, and students at Stonehill College, Kimberlee Marinelli and Amber Staples. Thank you all.

Advice, insight, feedback, and critiques were regularly provided by my thesis committee, for which I am deeply thankful. Dr. Ann Tarrant, thank you for reading and editing every manuscript and chapter. Dr. Anne Cohen, thank you for reminding me both that what I had was enough and what I wanted to do was too much. Dr. Roger Hanlon, thank you for asking the tough questions and making sure I had reason and justification for every action. Dr. Harry Hemond, thank you for catching the details and keeping me challenged on the physics and chemistry. Dr. Stefan Helmreich, thank you for the shift in perspective and for asking the big picture, socially impactful, synthesis questions.

Guidance in this project came from many others as well. Immense thanks to Dr. Dan McCorkle for chairing my proposal defense, for the use of many of your tools, and for the guidance and assistance to run and understand my carbonate system. Further thanks to Meredith White, Hannah Barkley, and members of the McCorkle lab past and present: Kathryn Rose, Elizabeth Bonk, Katherine Hoering, and Jennie Rheuban for help with carbonate system management and measurements. Drs. Andy Solow and Andy Beet were always willing to provide statistical and coding advice, for which I am very grateful.

System support came also from the excellent WHOI facilities staff at the Environmental Systems Laboratory, led by Rick Galat. I also want to deeply thank David Remsen, the staff of the MBL Marine Resources Center, and the crew of the Gemma for all of their assistance in getting squid over the years.

The MIT-Woods Hole Oceanographic Institution Joint Program would not exist without the efforts of a great many people, whose constant work and support allowed me to achieve this $\mathrm{PhD}$. I would like to thank the Academic Programs Office and its entire staff, including Deans Meg Tivey and Jim Yoder, Delia Oppo, Julia Westwater, Lea Fraser, Christine Charette and everyone who keeps this program running. I'd further like to recognize the constant efforts of Kris Kipp at MIT in this regard. Further I'd like to thank staff at WHOI, Gaynor Andrews and Muiread Pabalis, for their assistance throughout my time at the institution.

I also want to be sure to highlight and show my deepest appreciation to Dr. Ann Tarrant and Dr. Becky Gast for not only their times serving as Education Coordinator in the Joint Program \& Chair of the Joint Program Committee for Biological Oceanography, but for helping me manage and survive some of the most turbulent and difficult moments of this $\mathrm{PhD}$.

I want to make special note of the guidance of two (then) postdocs that aided not only the success of my experiments, but also my survival in this PhD: Dr. Karen Kityu Chan and Dr. Amy Maas. Similarly, I'd like to acknowledge the friendship and guidance of Dr. Emily Moberg, my senior in the Joint Program.

Beyond this, there were many friends in WHOI to whom I am deeply grateful for their presence, camaraderie, and support. Many were part of the Mooney Lab at one time: Annamaria Izzi DeAngelis, Tammy Silva, Samantha Zacarias, Thea Pisani, Justin Suca, Jessie Doyle, Jessie 
Perelman, Amy van Cise, Frants Jensen, and Ian Jones. And I should say that beyond these specific people, the Mooney Lab was often filled with wonderful and excellent people. Many friends were at WHOI as part of the Joint Program: Deepa Rao, Laura Weber, Hanny Rivera, Megan May, Jennifer Panlilio, Chrissy Hernandez, Mara Freilich, Hannah Mark, Christopher Kinsley, and Sarah Rosengard. I'd like to thank the community and friends I made at Anytime Fitness in Falmouth, as well. There are many friends in Woods Hole I am likely overlooking in this writing, and I apologize, but do appreciate you and the time we spent/spend together.

Thanks also to friends not only within, but also beyond the time and space of this $\mathrm{PhD}$, both those I kept in touch with (Sarah Almahdali) and those I have not.

Within WHOI, I want to note the Communications Department (thanks Lonny and Ken), WHOI Broader Impacts Group, WHOI DAC, and WHOI GLOW; all of whom I thank for their communities, efforts and events. Communities of support outside of this Institution and this $\mathrm{PhD}$ came in many forms: the Cephalopod International Advisory Council and the entire cephalopod research community, the Gordon Research Conference Global Ocean Change Biology community, the scientific communication and outreach communities (shout out to Sarah McAnulty and Diana Li, my cephalopod outreach comrades).

In part, this program ended up being the right choice for me more for the move to Boston and the incredible friends I made and got to spend time with as a result: Maris Wicks and Rosemary Mosco. I am deeply thankful for all the time working at cafes and discussing science, comics, science comics, and science communication. I am also thankful I got to work with and become friends with Abby Howard through our tactile comic project.

I am thankful also for escapism, as it provided great respite during this $\mathrm{PhD}$. Often this came in the form of podcasts (My Brother, My Brother, and Me, the Adventure Zone, Says Who!, and the Flop House), YouTube channels (GameGrumps and Bon Appetit's Test Kitchen), Twitch streams (Critical Role and Day[9]), comics (the Wicked and the Divine), and books (by N. K. Jemisin, Brandon Sanderson, and Dr. Nnedi Okorafor). Thank you for keeping me sane.

Escapism in stories also came through the creation and exploration of worlds in Dungeons and Dragons with my friends. Thank you to Siri Spencer for being my best friend for over a decade now and to the rest of the Pieces of Eight crew, Andrea, Erin, Linsday, Deepa, Rob, and Max, for excellent adventures. Thanks to Peter and Annamaria DeAngelis for my second game as well.

Thanks to great mentors in undergrad: Dr. Rob Erdman, Dr. Joseph Kakareka, Dr. Greg Tolley, and Dr. Paul Backhouse. A special thank you to my Master's advisor Dr. Michael Lee Berumen for his supporting me joining this project and getting into the Joint Program. Thanks also to great inspirations: Hank Green, Neil Gaiman, Scott McCloud, Bill Nye, and Jacques Cousteau.

To my family, thank you for your support. My grandparents for instilling me with a spirit of exploration. My brother for years of academic discourse and debate. My mother, in particular, for her unwavering belief and support in all of my ideas, dreams, and pursuits.

And, finally, thank you, of course, to the squid. 


\section{TABLE OF CONTENTS}

$\begin{array}{ll}\text { Abstract } & 3\end{array}$

Graphical Abstract $\quad 5$

$\begin{array}{ll}\text { Acknowledgments } & 7\end{array}$

Table of Contents 11

$\begin{array}{ll}\text { List of Figures } & 13\end{array}$

$\begin{array}{ll}\text { List of Tables } & 15\end{array}$

Chapter 1, Introduction: High performing, fecund, plastic invertebrates: Squid, $\begin{array}{ll}\text { acidification, and global ocean change } & 17\end{array}$

$\begin{array}{lll}1.1 & \text { Ocean acidification as part of global climate change } & 18\end{array}$

$\begin{array}{ll}1.2 & \text { Cephalopods in an acidifying ocean } \\ 1.3 & 20\end{array}$

$\begin{array}{lll}1.3 & \text { Cephalopods under hypercapnia } & 22\end{array}$

1.4 Calcifying structures under OA: the squid statolith 24

$\begin{array}{lll}1.5 & \text { Swimming and navigating in acidified seas } & 26\end{array}$

1.6 Parentage, variability, and resilience in a high $\mathrm{CO}_{2}$ ocean 27

1.7 Doryteuthis pealeii \& OA: Where are we now? 29

$\begin{array}{lll}1.8 & \text { Dissertation overview } & 31\end{array}$

Chapter 2: Dose-dependence and small-scale variability in responses to ocean acidification during squid, Doryteuthis pealeii, development $\quad 35$

$\begin{array}{ll}\text { Abstract } & 36\end{array}$

Introduction $\quad 37$

Materials and Methods $\quad 39$

Results $\quad 50$

$\begin{array}{ll}\text { Discussion } & 60\end{array}$

Chapter 3: Ocean acidification responses in paralarval squid swimming behavior using a novel 3D tracking system $\quad 81$

Abstract $\quad 82$

Introduction $\quad 83$

Materials and Methods $\quad 86$

Results $\quad 96$

$\begin{array}{ll}\text { Discussion } & 99\end{array}$

Chapter 4: Interannual and seasonal variability in the response of squid embryos and paralarvae to ocean acidification 
$\begin{array}{lr}\text { Abstract } & 122 \\ \text { Introduction } & 123 \\ \text { Materials and Methods } & 126 \\ \text { Results } & 130 \\ \text { Discussion } & 132\end{array}$

Chapter 5: Antagonistic interactions and clutch-dependent sensitivity induce variable responses to ocean acidification and warming in squid (Doryteuthis pealeii) embryos

Abstract

Introduction

Materials and Methods

Results

Discussion

Chapter 6, Conclusion and Future Directions: Early squid development under acidification: advances to the conceptual model following this dissertation

$\begin{array}{lll}6.1 \text { Summary } & 190\end{array}$

6.1a The conceptual model when we started 191

6.1b Dissertation findings 192

6.1c The conceptual model after this dissertation 196

$\begin{array}{lll}6.2 & \text { Future directions } & 197\end{array}$

$\begin{array}{lll}6.3 & \text { Broader implications } & 198\end{array}$

Appendix A: Chapter 2 Supplementary Materials 199

Appendix B: Chapter 3 Supplementary Materials 227

Appendix C: Chapter 4 Supplementary Materials 247

Appendix D: Chapter 5 Supplementary Materials 251

$\begin{array}{ll}\text { Bibliography } & 257\end{array}$ 


\section{LIST OF FIGURES}

Chapter 2: Dose-dependence and small-scale variability in response to ocean acidification during squid, Doryteuthis pealeii, development

$\begin{array}{lll}\text { Figure } 1 & \text { Metrics measured on paralarva images. } & 70\end{array}$

Figure $2 \quad$ Statolith morphometrics.

Figure $3 \quad$ Dorsal mantle length and yolk volume data by trial. 73

Figure 4 Dorsal mantle length and yolk volume data by hatching day. 74 - 75

Figure $5 \quad$ Aug 7 trial data by cup.

Figure 6 Comparisons of yolk volume and dorsal mantle length data. 77

$\begin{array}{lll}\text { Figure } 7 & \text { Cumulative percent hatching curves. } & 78\end{array}$

$\begin{array}{lll}\text { Figure } 8 & \text { Statolith morphometric data. } & 79\end{array}$

Chapter 3: Ocean acidification responses in paralarval squid swimming behavior using a novel 3D tracking system

Figure 13 arena set up and model system.

Figure 2 Proportion of time spent in top depth bin. $112-113$

Figure 3 Vertical swimming profile for a 400 ppm paralarva. $114-115$

Figure 4 3D path, polygon, tortuosity, and turn distribution of a paralarva. 117

Figure $53 \mathrm{D}$ velocity at various $\mathrm{CO}_{2}$ treatments across 2 minute recordings. $118-119$

Chapter 4: Interannual and seasonal variability in the response of squid embryos and paralarvae to ocean acidification

Figure 1 Visual summary of metrics measured across acidification experiments.

Figure 2 Acidification response data for all metrics and years.

Figure 3 Response ratio data compiled by year and across experiment timeline.

Figure 4 Seasonal data: baseline state change and response ratio variability.

$148-149$

Figure 5 Principal components analysis of response ratio data.

Figure 6 Comparative regressions of response ratio metrics.

Chapter 5: Antagonistic interactions and clutch-dependent sensitivity induce variable responses to ocean acidification and warming in squid (Doryteuthis pealeii) embryos

Figure $1 \quad$ Mantle length and yolk volume data and comparisons. $180-181$

Figure 2 Distributions of mantle length for individual Sep 14 egg capsules. 182

Figure 3 Hatching time data by trial. 183

Figure 4 Embryonic survival data by treatment and trial. 185

$\begin{array}{llr}\text { Figure } 5 & \text { Malformation images and data by treatment and trial. } & 186-187\end{array}$ 
Appendix A: Chapter 2 Supplementary Materials

Figure S1 Acidification and egg culture system.

$\begin{array}{lll}\text { Figure S2 Statolith area compared with dorsal mantle length. } & 205\end{array}$

Figure S3 Sample set of shapes for rugosity metric analysis. 213

Figure S4 Relationships between metrics and number of eggs per capsule. $224-225$

Appendix B: Chapter 3 Supplementary Materials

Figure S1 Proportion of time active over 10 minutes in 3D arena. 228

Figure S2 Dimensions of both the 2D and 3D arenas.

Appendix C: Chapter 4 Supplementary Materials

Figure S1 Response ratios, by year, plotted against 2 year average temperature anomaly.

Figure S2 Response ratios, by trial, plotted against either the 1 or 2 year average temperature anomaly based on lay date.

Appendix D: Chapter 5 Supplementary Materials

Figure S1 Distributions of mantle length for individual Jun 19 egg capsules.

Figure S2 Distributions of mantle length for individual Jul 28 egg capsules. 


\section{LIST OF TABLES}

Chapter 2: Dose-dependence and small-scale variability in response to ocean acidification during squid, Doryteuthis pealeii, development

Table 1 Seawater chemistry measurements and number of samples taken.

Table 2 Mantle length and yolk volume ANOVA table.

Chapter 3: Ocean acidification responses in paralarval squid swimming behavior using a novel 3D tracking system

Table $1 \quad$ Seawater chemistry and number of paralarvae per experiment. 106

Table 2 Dunn's tests for vertical depth bins. 107

Table $3 \quad$ Variance of vertical depth bins across $\mathrm{CO}_{2}$ treatments. 107

Table 4 3D metric data with Kruskal-Wallis tests and linear regressions. 108

Table 5 Variance and coefficient of variation of 3D metrics. 109

$\begin{array}{lll}\text { Table } 6 & \text { Significant Dunn's tests for 3D metrics. } & 110\end{array}$

Chapter 4: Interannual and seasonal variability in the response of squid embryos and paralarvae to ocean acidification

Table $1 \quad$ Summary of experiments and calculated $\mathrm{CO}_{2}$ treatments. 140

Table 2 ANOVA table for mantle length, hatching time, and yolk volume data. 141

Chapter 5: Antagonistic interactions and clutch-dependent sensitivity induce variable responses to ocean acidification and warming in squid (Doryteuthis pealeii) embryos

Table 1 Seawater chemistry and experimental metadata (number of samples). 178

Table 2 ANOVA table for mantle length and yolk volume data.

Appendix A: Chapter 2 Supplementary Materials

Table S1 ANOVA table of mantle length and yolk volume data by $\mathrm{pCO}_{2}$ treatment. $\quad 200$

Table S2 ANOVA table of mantle length and yolk volume data by hatching date. 201

Table S3 ANOVA table of mantle length and yolk volume data by cup. 202

Table S4 Hatching success distributions and G-tests. 203

Table S5 Resolution test of rugosity metrics: ranked subsets of shapes. 214

Table S6 Resolution test of rugosity metrics: ranked shape series. 215

Table S7 ANOVA of all metrics and variables, including egg number. $\quad 221$

Table S8 ANOVA of mantle length and yolk volume data by $\mathrm{pCO}_{2}$ and egg number. 222

Appendix D: Chapter 5 Supplementary Materials

Table S1 Hatching success distributions and G-tests. 252

Table S2 Malformation distributions and G-tests. 253 


\section{Chapter 1}

\section{Introduction}

High performing, fecund, plastic invertebrates:

Squid, acidification, and global ocean change 


\subsection{Ocean acidification as part of global climate change}

Ocean acidification (OA), the decrease in $\mathrm{pH}$ and concurrent carbonate equilibrium shift that results from the ocean absorbing anthropogenic atmospheric $\mathrm{CO}_{2}$, has been shown to have a wide variety of effects on a wide variety of marine taxa (Fabry et al. 2008; Vézina and HoeghGuldberg 2008; Kroeker et al. 2010, 2013; Dorey et al. 2013; Parker et al. 2013). On its own, OA is known to impact calcification, acid/base regulation, neurotransmission, and developmental processes, all potentially affecting the energy budget of an organism (Gazeau et al. 2007; Gutowska et al. 2008; Hu et al. 2011a; Nilsson et al. 2012; Dorey et al. 2013; Chung et al. 2014). Some of these effects can be compensated for in the absence of other stressors or in an energyreplete environment. When these two conditions are not satisfied, OA is notorious for amplifying the overall physiological impact of other stressors (Findlay et al. 2010; Rodolfo-Metalpa et al. 2011; Rosa et al. 2013, 2014a). Despite some evidence of antagonistic relationships with some stressors, OA has primarily developed a reputation as the stressor that will compound the rest (Kroeker et al. 2010, 2013; Breitburg et al. 2015). In order to understand how organisms and communities will respond in this rapidly changing environment, we must determine the physiological and behavioral effects that OA induces, and the scales on which they vary, in order to gain insights into the possible mechanisms underlying stress effects.

Certain organisms will be better adapted than others to OA; only through experimentation can we identify the potential "winners" and "losers." Calcifiers are known to fight an uphill energetic battle against a dropping aragonite saturation state (Cohen and Holcomb 2009; Lunden et al. 2014). As pH drops, it becomes harder for calcifiers to maintain concentration gradients within calcifying spaces (Gazeau et al. 2007; Dissard et al. 2009; Ries et al. 2009; RodolfoMetalpa et al. 2011). Non-calcifiers face similar threats during acidosis and require energy to maintain a proper, functional $\mathrm{pH}$ that enables maintenance and activity of proteins in intra- and extracellular fluids (Pörtner et al. 1998; Langenbuch and Pörtner 2002; Michaelidis et al. 2005; Gutowska et al. 2010a). The limits of processes such as calcification and homeostasis are set from an organism's evolutionary and life history, which dictate the optimal conditions under which the organism can use these processes and still maximize its energy budget for survival and reproduction (Parmesan 2006; Hu et al. 2011a; Sunday et al. 2014). Research into these physiological limits provides insight into which species are best built to survive these rapidly oncoming stressors. 
Strong performance under stress as an adult does not indicate complete adaptation; all stages of development must be considered. Adult marine organisms, particularly pelagic species, are considered more resilient to stressors due to their mature organ systems and an ability to avoid stress through behavioral modification (Wannamaker and Rice 2000). In contrast, marine eggs and larvae can be left to the devices of the surface currents of the pelagic or tied to the benthos (Vecchione 1981; Robin et al. 2014). These stages are often thought to be more susceptible to stress because they are still actively developing the organ systems needed to mitigate impacts (Findlay et al. 2010; Nakamura et al. 2011; Ross et al. 2011; Nguyen et al. 2012; Kaplan et al. 2013). A "winner" built to deal with a range of $\mathrm{pH}$ in the adult phase may not be as well constructed to handle chronic or periodic acidosis in its early life history (Kaplan et al. 2013; Munday et al. 2011; Munday et al. 2009). Although there is reason to believe that embryos, given a high activity of developmental machinery, are conditioned to natural environmental variation, it is uncertain whether that flexibility extends to the extremes of anthropogenic changes (Hamdoun and Epel 2007). Impacts to early development in the eggs and larvae can have far-reaching effects on the population dynamics of any organism (Cowen and Sponaugle 2009; Munday et al. 2009a).

Morphology, physiology, and behavior are often the more directly observable responses of an organism under stress, which can act as the starting point to not only understanding the species' stress response, but also to formultating hypotheses on what mechanisms drive those impacts (Cohen and Holcomb 2009; Hendriks et al. 2015). Environmental stressors, like acidification, operate across spatial and temporal scales of organization, altering molecular interactions while simultaneously disrupting global geochemical cycles (Vézina and HoeghGuldberg 2008; Doney et al. 2009). Morphology, physiology, and behavior are the core from which one can bridge the molecular to the ecological. Data at this scale can provide fundamental information for the construction of predictive models (Cheung et al. 2008, 2009). However, anthropogenic stress studies are often run as single sets of experiments, with results being interpreted as representative of general principles for ecophysiology in the taxa when the reality we are attempting to predict is much more complex (Kroeker et al. 2017). While understandably rare due to logistical issues, it is important that longer term, repeated studies of organismal stress response are performed in order to develop a better understanding of how variable these responses can be. 


\subsection{Cephalopods in an acidifying ocean}

Cephalopods are a unique group of marine organisms. They are unparalleled among marine invertebrates in the diversity and complexity of their behaviors and physiology: they are as dynamic as they are inconsistent (Robin et al. 2014). Recent work has begun to address potential OA impacts on some cephalopod taxa. Cuttlefish have often been studied for OA response, because their remnant shell, the aragonitic cuttlebone, is a prominent feature and has been shown to hypercalcify under low pH (Gutowska et al. 2010b; Dorey et al. 2013). Adults and juveniles of the jumbo squid, Dosidicus gigas, are the only relatively well studied oegopsid squid under hypercapnia, with early data suggesting metabolic suppression, but more recent work showing a consistent ability to oxygenate blood and tissue under acidification (Rosa and Seibel 2008; Birk et al. 2018). Little to no work has been done with octopuses, nautilus, spirulids, sepiolids, or many of the rare deep-water members of the taxa due to the logistical difficulty of acquiring and managing representatives of these groups.

Several species of coastal myopsid squid have shown to be impacted, especially at the embryonic and paralarval phase (Kaplan et al. 2013; Navarro et al. 2014, 2016; Rosa et al. 2014b). These squids are a core component of coastal food webs and are also a human-harvested protein source, serving as the foundation of valuable fisheries (Rosa et al. 2014b; NOAA 2019). They are a relatively accessible and manageable group within the cephalopods, which makes robust, replicated experimentation in stress response studies feasible, providing the foundation to assess taxon level patterns.

Myopsid squid begin their lives as eggs tethered to the benthos after which they hatch as paralarvae (Vecchione 1981). Navarro et al. demonstrated that the potential laying environment for egg capsules can be highly dynamic in terms of $\mathrm{pH}$ and $\mathrm{O}_{2}$ on the Pacific Coast due to episodic upwelling $(2014,2018)$. Taxonomically conserved adaptation to widely varying $\mathrm{pH}$ and oxygen laying environments, such as the Pacific Coast upwelling system or estuarine outlets, may allow for a wider range of tolerances to fluctuating and/or extreme conditions (Jacobson 2005; Rosa et al. 2014b). Excluding the environmental variability of the laying system, microprobe $\mathrm{pH}$ profiles of developing egg masses indicate that even under ambient $\mathrm{CO}_{2}$ conditions, pre-hatching paralarvae are brought to the brink of oxygen deprivation and severely reduced $\mathrm{pH}$ (Long et al. 2016). While this indicates that existing machinery must already be in place to cope or endure under some amount of acidosis and hypoxia, there is also a strong 
potential that any further decreases in $\mathrm{pH}$ or $\mathrm{O}_{2}$ in this already highly strained system may tip it over the edge of resilience.

Squid are highly active and competitive pelagic predators that are programmed to survive by operating by a "live fast, die young" lifestyle (O’Dor and Webber 1986; Bartol et al. 2009a). High activity levels entail high oxygen requirements. For active squid, this means exploiting the carrying capacity of their blood pigment, hemocyanin, to quickly load and offload gases (Seibel 2013). Therefore, the $\mathrm{pH}$ sensitivity of the hemocyanin is an important component of a squid's ability maintain normal activity (Pörtner 1990; Zielinski et al. 2001). Most adult cephalopods are capable of compensating for $\mathrm{pH}$ stress by upregulating the amount and/or activity of ionexchanging protein pumps within the inner gill membrane (Hu et al. 2011a). Representatives of several types of squid, D. gigas, D. pealeii, Sepioteuthis lessoniana, and Idiosepius pygmaeus, have shown no effect of acidification on oxygen transport and aerobic performance (Birk et al. 2018; Spady et al. 2019). Cuttlefish are known to express different isoforms of hemocyanin in different proportions across different life stages, but this process was shown to be strongly driven by ontogeny rather than the presence of stressors (Strobel et al. 2012). Compensatory expression and regulation of proton secretion pathways under acidification has been demonstrated in the developing embryos of bigfin reef squid, S. lessoniana (Hu et al. 2013). It is possible that the naturally acidifying environment of the intracapsular fluid has conditioned developing embryos to transcribe and express $\mathrm{pH}$-compensating proteins, but this mechanism has not been robustly explored across the cephalopods.

Cephalopods retain aragonitic structures beyond just the cuttlebone of cuttlefish: statoliths, the calcium carbonate core of the statocyst sensory system, are a vital part of these organisms' ability to orient and swim (Arkhipkin and Bizikov 2000). Statoliths are formed within a calcifying membrane within the fluid-filled sac of the statocyst (Lipinski 1993). Environmental conditions, particularly surrounding chemistry, can have a strong impact on the development of both the statolith as well as the statocyst, which can translate into aberrant paralarval swimming behaviors (Colmers et al. 1984). Stress checks, dark band deposited during stress, are a common feature in several of the incrementally deposited structures (e.g. beaks) found in cephalopods, suggesting these processes are particularly susceptible under metabolic strain (Perales-Raya et al. 2014; Franco-Santos et al. 2016; Jin et al. 2019). Neither the 
mechanisms behind these impacts nor the effects on paralarval survival in a natural setting are clear; this organ's sensitivity, however, suggests it is a strong candidate to signal active stressors.

Squid occupy a wide, pelagic ecological niche, competing with teleost fishes through strategies that maximize physiological demand (O’Dor and Webber 1986; Coll et al. 2013; Navarro et al. 2013). Between their early link to the benthos and this energetically strained neritic existence, OA has the potential to inflict stress at various levels of myopsid squid physiological organization. There is therefore a strong need for additional and extensive squid OA experiments in order to inform predictions of impacts, our potential management of this taxon, and subsequently, the trophic networks that depend on it.

\subsection{Cephalopods under hypercapnia}

Early work on squid exposed to elevated $\mathrm{CO}_{2}$ focused on adult respiratory physiology under extreme hypercapnia (Redfield and Goodkind 1929; O’Dor and Webber 1986; Shadwick et al. 1990; Pörtner et al. 1991). Redfield \& Goodkind (1929) exposed adult D. pealeii to pressures of $\mathrm{CO}_{2}$ as high as $20 \mathrm{mmHg}$ (greater than 25,000 ppm) to explore changes in oxygen consumption, lethal dosages, and hemocyanin carrying capacity. They concluded that stress and death of adult squid under hypercapnia was a result of the corresponding decrease in oxygen partial pressure and not an impact of the parallel decrease in seawater acidity (Redfield and Goodkind 1929). Work on squid activity and respiration has demonstrated that these animals operate at the very edge of their energetic capacity at all times, although more recent work has shown a capacity for oegopsid squids to reduce their metabolic activity in low $\mathrm{O}_{2}$, high $\mathrm{CO}_{2}$ environments (O’Dor and Webber 1986; Webber and O’Dor 1986; Shadwick et al. 1990; Pörtner et al. 1991; Rosa and Seibel 2010; Webber et al. 2010; Seibel 2013; Trueblood and Seibel 2013; Seibel et al. 2014).

Oxygen physiology remained the focus of cephalopod $\mathrm{CO}_{2}$ research until climate change became prominent, after which it followed the paradigm shift in marine science into ocean acidification and its potential for impacts on the early life stages of many organisms (Gazeau et al. 2010; Byrne 2011; Stumpp et al. 2011; Lischka et al. 2011; Nakamura et al. 2011; Appelhans et al. 2012; Maas et al. 2012; Pansch et al. 2012; Seibel et al. 2012; Parker et al. 2013; Rivest and Hofmann 2014). Seibel et al. (2013) bridged these paradigms by focusing on metabolic depression of juvenile Humboldt squid, D. gigas, under oxygen minimum zone (OMZ) linked 
hypoxia and hypercapnia and related these conditions to ocean acidification with concerns that the $\mathrm{pH}$ sensitive hemocyanin may become less effective. Juvenile and adult D. gigas have been shown to decrease ATP demand and oxygen consumption, increase octopine, a product of anaerobic respiration, and globally inhibit transcription and translation in order to conserve energy while in OMZ levels of hypoxia (Seibel et al. 2014). While this physiology is advantageous for D. gigas as a competitive predator, in light of expanding OMZ's, there was concern these reductions in blood pigment carrying capacity, routine metabolic rate, and ATP production may quickly become disadvantageous in light of concurrent warming and acidification (Rosa and Seibel 2008, 2010).

Further work has shown, contrastingly, that adults and juveniles of both D. gigas and D. pealeii do not reduce metabolism or critical oxygen partial pressure under increased acidification; models indicate these animals are unlikely to be pushed to their oxygen limits even under future predictions for OA (Birk et al. 2018). A similar situation has been seen in $S$. lessoniana, with adults exposed to hypercapnia demonstrating a 40\% metabolic depression, but an increase in mRNA and protein levels concurrent with compensation through active bicarbonate and ammonium transport (Hu et al. 2014). Further experiments with this species have shown no impacts on this species' oxygen uptake rates and aerobic scope (Spady et al., 2019). Sepia officinalis consistently demonstrates an increase in calcification activity across life history stages under decreased pH resulting in larger cuttlebones (Gutowska et al. 2008; Gutowska et al. 2010; Dorey et al. 2013; Sigwart et al. 2014). Despite the extreme low pH/low $\mathrm{O}_{2}$ concentrations in the perivitelline fluid that drive this hypercalcification response during embryonic development, observations of acidification impacts on growth in developing $S$. officinalis have been uncertain and variable (Gutowska and Melzner 2009; Gutowska et al. 2010b; Sigwart et al. 2016). Contrasting results such as these may to some extent suggest that there exists variability either between experiments or within a species that are not being accounted for.

While less work has been done to examine metabolic and aerobic activity of developing cephalopod embryos under acidification, studies with the European squid, Loligo vulgaris, have shown reductions in oxygen consumption rate under acidification (Rosa et al. 2014a). A comparison of late stage embryos and adults of $S$. officinalis exposed to increased $\mathrm{CO}_{2} \mathrm{saw}$ a clear reduction in somatic growth and a downregulation of ion transporters and metabolic genes 
only during early life, demonstrating a potential for prominent sensitivity for cephalopods in this phase (Hu et al. 2011). Eggs of both S. officinalis and Loligo vulgaris demonstrated an increased permeability to trace metals when raised under acidified waters (Lacoue-Labarthe et al. 2011, 2012). Rosa et al. exposed eggs of $S$. officinalis to both acidification and warming and observed a decrease in hatching time and survival along with an increase in premature hatching (2013). This study also noted a temperature independent depression of the cuttlefish embryos' energy expenditure as a result of increased acidification, contributing to their impeded growth (Rosa et al. 2013). A similar study, examining the impact of only warming on the early life history of squid, L. vulgaris, demonstrated metabolic suppression, increased premature hatching, and a strong response by the animals' oxidative stress pathways (Rosa et al. 2012). It has been suggested that the early life stages of squid are more sensitive than those of cuttlefish or octopuses, particularly to acidification stress, due to their high energy lifestyle (Hu et al. 2010).

Research into impacts of ocean acidification on the early life history of squid was first done by Kaplan et al. with D. pealeii, the precursor to this thesis (2013). This study demonstrated delayed growth, reduced mantle length, and degraded statoliths under elevated $\mathrm{CO}_{2}(2200$ ppm)(Kaplan et al. 2013). Rosa et al. have examined the combined impacts of increased $\mathrm{CO}_{2}$ and warming on embryos of $L$. vulgaris laid at different times of year and saw decreased oxygen consumption, increased premature hatching and malformations, and a decrease in thermal tolerance limits in embryos due to hypercapnia, particularly in the summer progeny (2014b). Squid embryos laid in summer were also seen to have a $47 \%$ survival rate under combined hypercapnia and warming, whereas the winter progeny's survival was unaffected; however it should be noted that experiments in this study involved the removal of embryos from the egg capsule prior to being raised in stressful conditions, which may have impacted their survival (Rosa et al. 2014a). Paralarvae of another myopsid squid, Doryteuthis opalescens, have been examined under OA and decreased oxygen with a focus on finding biomarkers for hypoxic and hypercapnic exposure histories, demonstrating, so far, a potential low-oxygen signal via the uranium to calcium ratio of their statoliths (Navarro et al. 2014; Levin et al. 2015).

\subsection{Calcifying structures under OA: the squid statolith}

Calcification of aragonite under ocean acidification is a prominent research field, focusing primarily on corals and shellfish, but has begun expanding into other marine organisms 
(Gazeau et al. 2007; Fabry et al. 2008; Gutowska et al. 2008; Dissard et al. 2009; Ries et al. 2009; Rodolfo-Metalpa et al. 2011; Fernández-Reiriz et al. 2012; Comeau et al. 2015). The precipitation of aragonite can be affected by OA through reduction in available carbonate ions, an increase in the rate of dissolution of calcium carbonate structures due to decreased $\mathrm{pH}$, or suppression of organismal metabolism resulting in reducing rates of calcification (Fabry et al. 2008; Cohen and Holcomb 2009). Furthermore, pH driven decreases in aragonite saturation state in the calcifying space can alter crystal size and structure, while simultaneously altering the activities and morphology of calcifying matrix proteins (Holcomb et al. 2009, 2014; Tambutté et al. 2015). Elemental ratios are often used as proxies for determining the physical and chemical nature of the calcifying fluid; $\mathrm{U} / \mathrm{Ca}$ demonstrates a shift in carbonate ion concentration, for example (Gaetani and Cohen 2006; Navarro et al. 2014; DeCarlo et al. 2015b). However, factors affecting calcification under reduced $\mathrm{pH}$ can be variable across species (Ries et al. 2009). Elemental ratios can be affected by the nutritional state, growth rate, and calcification rate of the organism, so care must be taken in interpretation of geochemical proxies to determine OA effects (Carré et al. 2006; Freitas et al. 2006; Gaetani and Cohen 2006; Takesue et al. 2008; Holcomb et al. 2010; Drenkard et al. 2013; DeCarlo et al. 2015a).

Squid statoliths are one of several calcium carbonate structures produced by cephalopods, but thus far only Kaplan et al. have shown potential degradation of this aragonitic sensory apparatus due to OA (2013). The cuttlebones of $S$. officinalis have been shown to maintain or hypercalcify under decreased $\mathrm{pH}$; a response also seen commonly in fish otoliths (Gutowska et al. 2008; Checkley et al. 2009; Munday et al. 2011; Dorey et al. 2013). Argonaut (a type of pelagic octopus, sometimes called a paper nautilus) egg cases showed marked degradation in decreasing $\mathrm{pH}$ treatments, a result of their direct exposure to the surrounding waters, but notably no change in their mineralogy was seen (Smith et al. 2012). Jellyfish statoliths, a calcium sulfate analog to squid statoliths, have been seen to decrease in size in low $\mathrm{pH}$, similar to the results seen by Kaplan et al., but these reductions were tied entirely to metabolic suppression and no structural or elemental analyses were performed (2013; Winans \& Purcell 2010). Statoliths are laid down in sequential layers, so the bulk of statolith analyses are used for aging and/or determination of physical and chemical exposure history of the organism (Lipinski 1993; Arkhipkin and Bizikov 1997, 2000; Arkhipkin 2003; Villanueva et al. 2003; Navarro et al. 2014; Levin et al. 2015). Current methods for the analysis of statolith degradation are fairly qualitative 
and do not address the mechanisms or points in development where stress impacts occur; development or utilization of more robust techniques could provide greater insight into the process of calcification stress in cephalopods.

\subsection{Swimming and navigating in acidified seas}

Research into OA effects beyond calcification and physiology have been slow to emerge and have focused primarily on neuroethology, especially olfactory control of swimming orientation and homing, rather than the mechanics of locomotion (Ferrari et al. 2011; Dixson et al. 2010; Munday et al. 2013; Nilsson et al. 2012; Munday et al. 2009; Dixson et al. 2015). A large body of work is emerging examining effects of OA on sea urchin larvae showing that morphological shifts driven by low $\mathrm{pH}$ can inhibit larval activity and change swimming dynamics (Dupont et al. 2010; Martin et al. 2011; Chan et al. 2013, 2015). Although several studies demonstrating cuttlebone and otolith hypercalcification have been produced, there have been few notable observations of impacts or in-depth examinations of the corresponding organism's swimming dynamics (Checkley et al. 2009; Munday et al. 2011; Dorey et al. 2013; Maneja et al. 2013; Pimentel et al. 2014b; Réveillac et al. 2015). Pimentel et al. (2014) exposed larval dolphinfish, Coryphaena hippurus, to OA and saw decreased swimming duration and orientation frequency in elevated conditions (1600 ppm), but did not examine the larval otoliths, tying the observed locomotor shifts to metabolic and activity depression.

Studies of squid paralarval swimming dynamics have primarily focused on the high energy demand and low efficiency of the pulsed jet swimming mode on which they rely (Bartol et al. 2008, 2009a; Thompson et al. 2010). Paralarval swimming in a broad sense is incorporated into studies using models to examine dispersal and biogeography of squids in this life stage, noting their propensity to swim to the surface at hatching and perform diel migrations for feeding, driven in part by their dynamic photopositivity (Barón 2003; González et al. 2005; Moreno et al. 2008; Shea and Vecchione 2010). Few studies have directly explored squid statolith quality in concert with swimming behavior, despite the statolith's key role in cephalopod orientation, though the work tying "spinner" cephalopods to strontium availability stands out (Colmers et al. 1984; Hanlon et al. 1989; Arkhipkin and Bizikov 2000). Although changes to squid statolith quality and elemental composition have been observed under 
acidification (Kaplan et al. 2013; Navarro et al. 2014), no experiments have examined paralarval swimming under OA.

Sensory responses to ocean acidification have recently become a major field of study in fish, larvae in particular, with the change in ocean chemistry caused by acidification affecting neurological pathways of olfaction and vision (Dixson et al. 2010, 2015; Munday et al. 2013, 2014; Chung et al. 2014). Decreased pH and increased bicarbonate in the seawater chemical environment have a notable effect on the neurotransmission of sensory signals in several reef fishes (Nilsson et al. 2012; Chung et al. 2014). This pathway has very recently been shown to even impact the visual system of damselfish through reduced retinal flicker frequency (Chung et al. 2014).

Broadly, squid are well known for their intense photopositivity and it is a popular subject in squid fisheries research (Campus 1999; Semmens et al. 2007). Most squid rely on light for communication, through both microbial-associated photophores and/or iridescence and chromatophores (Ruby and McFall-Ngai 1992; Kingston et al. 2015). Although positive phototaxis is a prominent sensory-related behavior in many organisms, few studies have used it as a variable for stress responses (Aiken and Man 1978; Markow 1979; Flickinger et al. 1982). Munday and colleagues lead the charge in OA effects on sensory responses, behaviors, and neurology, and have begun to examine OA-influenced behavioral shifts in adult pygmy squid (a sepiolid, not a teuthid), I. pygmaeus, noting increased activity and defensive behaviors (Spady et al. 2014). Better integration of sensory and behavioral methodologies in the research of stress responses may be a rich vein from which to mine for both underlying mechanisms and ecological interactions.

\subsection{Parentage, variability, and resilience in a high $\mathrm{CO}_{2}$ ocean}

The impacts of parental exposure and condition on their offspring are a growing focus in stress ecology as ideas from genetics, epigenetics, and developmental physiology converge. Parental effects in concert with OA have been demonstrated in an anemonefish, Amphiprion melanopus, with offspring from OA-exposed parents proving more resilient (Miller et al. 2012). Larvae of another species of anemonefish, Amphiprion percula, also demonstrated variation in response to OA dependent on parentage, but with no parents having been exposed to OA in this experiment, indicating a genetic effect (Munday et al. 2009). More broadly, non-genetic effects 
of parental condition have been seen in another coral reef fish species, Acanthochromis polyacanthus, with better conditioned parents producing larger offspring with greater energy reserves (Donelson et al. 2008). Murray et al. have demonstrated that parental effects in fishes can affect OA sensitivity of offspring in seasonal patterns by changing the exposure conditions of the parents (2014). It is clear from this body of research that, at least in marine fishes, parental exposure and condition can act as an important driver of early offspring success, particularly in stressful environments (Donelson et al. 2009).

The impacts of parentage have also been demonstrated in invertebrates, with transgenerational acclimatization to OA and climate change becoming a prominent focus in corals (Putnam and Gates 2015), but little has been done on the topic with cephalopods. van der Sman et al. (2009) demonstrated an impact of maternal input on the early success of snail, Cominella virgata, offspring, with improved growth seen in the first month for larvae of better fed mothers regardless of nutritional condition. The presence of these effects in other mollusks and in fishes, to which squids are often similar due to competition pressures, indicate a promising potential for similar effects in squid.

Squid populations are known to vary on seasonal and interannual scales as their environment impacts their growth directly, through temperature and water chemistry, and indirectly, through trophic cascades (Yatsu et al. 2000; Pecl et al. 2004a; Pecl and Jackson 2008; Keyl et al. 2011). Many squids live about 1-2 years, producing either year classes or seasonal cohorts depending on the breeding window for the species (Arnold et al. 1974; Mesnil 1977; Hanlon and Messenger 1998; Jacobson 2005; Rosa et al. 2014a). However it is thought that these large single cohorts might be comprised of a succession of smaller cohorts, the dynamics of which have only been described in S. lessoniana (Arnold et al. 1974; Mesnil 1977; Moltschaniwskyj and Pecl 2007) As squid do not store energy, maternal investment depends on two major factors: how much energy the female has invested into reproductive rather than somatic growth over her lifetime, and how much immediately available energy she is able to harvest to invest into egg production (Steer et al. 2004; Pecl and Moltschaniwskyj 2006). Squid parentage is further complicated by the predominance of maternal sperm storage and multiple paternity (Buresch et al. 2001, 2009; Emery et al. 2001). While not explored in cephalopods in depth, variability between egg capsules has been demonstrated in the elemental makeup of statoliths in D. opalescens embryos (Navarro et al. 2014). These effects produce a situation 
where parental preconditioning and environmental variability could strongly effect the initial state of the squid embryo.

Once the initial state is set for the embryo, it is entirely the interaction of that genetic predisposition with the state of its surroundings that determines its trajectory in development. Temperature is known to be a major influence on embryonic squid, increasing development rate and producing smaller hatchlings (McMahon and Summers 1971; Pecl 2004; Leporati et al. 2007). Rearing temperaures impact not only initial size at hatching, but also growth rates of cephalopod paralarvae, which can translate to very different life histories, sizes at maturity, and cohort survival (Steer et al. 2003; Pecl et al. 2004b). A short lifespan, high fecundity, and high plasticity under environmental influence are advantages that allow squid and some other cephalopod populations to have relatively good flexibility and to respond quickly to environmental change, suggesting they may do very well under anthropogenic change (Doubleday et al. 2016). However, the full scope of these variabilities remain uncharacterized and so it remains uncertain what the limits of this adaptability may be and if cephalopod populations will be able to keep pace with anthropogenic stress (Pecl and Jackson 2008).

\subsection{Doryteuthis pealeii \& OA: Where are we now?}

Doryteuthis pealeii, the Atlantic longfin squid, is the primary myopsid market squid along the coast of the northwest Atlantic (Hatfield and Cadrin 2002; Buresch et al. 2006). It is a vital food source for many local and migratory fishes, birds, and mammals that make up the broad ecology of Vineyard Sound, MA, USA and offshore waters, and a notable fishery for humans (Jacobson 2005; Hunsicker and Essington 2006, 2008; NOAA 2019). The giant axon of D. pealeii has been extensively studied within the field of neuroscience. As a result, the biology, behavior, and ecology of this species have also been deeply studied (Summers 1971; Vecchione 1981; Macy III 1982; Hanlon and Messenger 1998; Herke and Foltz 2002; Jacobson 2005; Hunsicker and Essington 2008). However, less is known about this organism's early life history due to difficulty in culturing it past the paralarval stage and the relative scarcity of wild paralarval and juvenile studies (Vidal et al. 2002b; Jacobson 2005).

A wide ranged species, spanning from Nova Scotia through the Gulf of Mexico, $D$. pealeii is limited by latitude and depth primarily by temperature regime $\left(8-16^{\circ} \mathrm{C}\right.$ bottom temperature), restricting it to coastal shelves and an approximate maximum depth of 400 meters 
(Vecchione 1981; Hatfield and Cadrin 2002; Herke and Foltz 2002). As a squid that does not utilize oxygen minimum zones during its adult life and is not known to dive to extreme depths, it is not thought to seek out extreme shifts in water chemistry (Hatfield and Cadrin 2002).

However, it is found within estuarine systems suggesting a reasonable tolerance to salinity and oxygen variability (Jacobson 2005). Given its short maturation time ( $<1$ year) and high fecundity (50-300 offspring per egg capsule), it is a squid with a high potential for population plasticity and adaptability (Vecchione 1981; Macy III and Brodziak 2001).

Egg habitat for $D$. pealeii has not been as robustly characterized and mapped as it has for its northeast Pacific counterpart, D. opalescens (Navarro et al. 2018). Observations of deposited strings of egg capsules (often referred to as 'mops') along the northwest Atlantic shelf have broadly characterized $D$. pealeii egg habitat as constrained to $10-23^{\circ} \mathrm{C}$ and $30-32 \mathrm{ppt}$ (McMahon and Summers 1971; Jacobson 2005). Doryteuthis opalescens egg habitat is constrained to levels of oxygenation greater than $160 \mu \mathrm{mol}$ and of $\mathrm{pH}_{\mathrm{t}}$ greater than 7.8 (Navarro et al. 2018). Such metrics have not been measured in situ for $D$. pealeii egg capsules, but the northwest Atlantic is notably less oxygen variable than the northeast Pacific. Cruise-measured carbonate system profiles for the northwest Atlantic shelf suggest a potential exposure range of 8.2-7.88 $\mathrm{pH}_{\mathrm{t}}(250$ $600 \mathrm{ppm} \mathrm{pCO}_{2}$ ) at known egg laying depths during $D$. pealeii breeding season (values calculated using $\mathrm{CO}_{2}$ SYS with data from Wang et al., 2013). The embryos are encapsulated in a mucous package, which naturally acidifies and deoxygenates over development, becoming anoxic and reaching $\mathrm{pH}$ values as low as 7.34 prior to hatching (Long et al. 2016). This suggests that despite environmental influence, the embryos may be equipped to deal with some level of acidification during development, although the limits of this capability are unclear.

Preliminary work performed in 2012 demonstrated a notable potential for acidification impacts on the development and early life history of D. pealeii (Kaplan et al. 2013). Paralarvae raised in an elevated $\mathrm{CO}_{2}$ treatment (2200 ppm) showed increases in development time and reductions in mantle length, indicating a potential metabolic suppression or taxation on the energy budget of developing paralarvae (Kaplan et al. 2013). Statoliths dissected from high $\mathrm{CO}_{2-}$ exposed paralarvae demonstrated a reduction in size and overall quality, with a concurrent increase in porosity and misshapenness (Kaplan et al. 2013). The statolith effects suggested a potential for both a shift in the control mechanisms of internal $\mathrm{pH}$ within the statocyst and sensory impacts to swimming and orientation in response to acidosis. This preliminary work, as 
well as the literature provided by Rosa et al. (2014b) and Navarro et al. (2014, 2016), provide a groundwork from which to investigate acidification and its impacts on $D$. pealeii embryos more deeply, to try to determine the scope and limits of those impacts, and to develop hypotheses for the mechanisms underlying them.

\subsection{Dissertation overview}

This dissertation sought to robustly examine the morphological, physiological, and behavioral impacts of ocean acidification on the embryonic development and resultant hatchling parlarvae of the squid, Doryteuthis pealeii. I have sought to integrate data across multiple scales including behavior, morphology, development, energetics, physiology, and ecology. The research and advances described in this dissertation include:

- New methods developed to more quantitatively and robustly describe observed impacts to squid paralarvae morphology, physiology, and behavior

- Implementation of these novel and existing methods to determine the threshold and scope for variability in the sensitivity of Doryteuthis pealeii embryos to ocean acidification

- Evaluation of variability in stress response across temporal and biological scales: across hatching days, within a single experiment/clutch, across the breeding season, and across years

- Use of strong observations of stress responses under extreme conditions to develop new hypotheses for the mechanisms through which impacts occur

The chapters described here in include:

Chapter 2 - Dose-dependence and small-scale variability in responses to ocean acidification during squid, Doryteuthis pealeii, development

This chapter describes dose response experiments run in 2013, with squid eggs reared under a range of $\mathrm{pCO}_{2}$ concentrations (from 400 - $2200 \mathrm{ppm}$ ) and examined for impacts to embryonic development, survival, and morphology/physiology of the paralarvae. These data correspond to those reported previously as part of my Master's thesis (Zakroff 2013). However, further analyses have been performed with a more substantive statistical methodology that better 
supports the consistent observation of acidification impacts starting at around $1300 \mathrm{ppm} \mathrm{pCO}_{2}$. Several new methods for the quantitative analysis of statolith morphometrics and surface degradation were developed and are described as well, providing a new toolset for description of impacts to this structure. This chapter also delves into the small scale variability of responses. At the scale of days, I show that acidification stress can result in different dynamics of hatching as it progresses. Scaling up temporally, I also describe shifts in overall response intensity between clutches across the breeding season.

Chapter 3 - Ocean acidification responses in paralarval squid swimming behavior using a novel $3 D$ tracking system

Here, I developed new methodologies to record, track, and process videos of squid paralarvae swimming and apply them to analyzing the potential impacts of OA. This chapter uses data from paralarvae subsampled from the 2013 experiments described in Chapter 2 as well as from experiments throughout 2014 and at the start of 2015. In 2013, I used a 2D system, from which I describe acidification-driven decreases in the ability of squid paralarvae to maintain station near the surface of the chamber. Experiments in 2014 and 2015 used this novel 3D system, the model system and code for which I provide along with the experimental data. It was difficult to pull consistent patterns from the 3D data, as the paralarvae demonstrated intense individual variability in swimming patterns. However, I find that activity and velocities decrease with increasing acidification. In sum, these experiments suggest an impact of acidification on the energetics of swimming, and thereby of the paralarvae themselves. These data not only further enforce the small-scale variability discussed in Chapter 2, but also introduce larger scale variability, with effects shifting in intensity from 2013-2014/15, that is discussed in Chapter 4.

\section{Chapter 4 - Interannual and seasonal variability in the response of squid embryos} and paralarvae reared under ocean acidification

In this chapter, I examined patterns in response and sensitivity to acidification over the course of all four years of experiments, 2013-2016. First, I examined trends in the raw data across years. Then, I examined the seasonal trends in the baseline/control state of the embryos and paralarvae. In the bulk of this chapter, I used response ratios between the $2200 \mathrm{ppm}$ treatment (the only consistent concentration across all experiments) and lowest $\mathrm{pCO}_{2} /$ control 
treatments for each metric to compare responses across years. I found that there are consistent seasonal shifts in the baseline state of the paralarvae that appear to be driven by parental conditioning from ambient environmental temperatures. I also found that OA sensitivities seem to vary independently of this baseline state and are driven more by the year-class/cohort of the parents, although this also may be linked to their environmental history. This chapter highlights the importance of robust replication in global ocean change stress studies in order to get a fuller picture of both the scope of sensitivity of a species/population and the drivers of variability that influence that sensitivity.

\section{Chapter 5 - Antagonistic interactions and clutch-dependent sensitivity induce variable} responses to ocean acidification and warming in squid (Doryteuthis pealeii) embryos

Here, I exposed squid eggs not only to increased acidification, but warming as well, rearing the eggs at $+2{ }^{\circ} \mathrm{C}$ above peak temperatures for Vineyard Sound, MA, USA. This chapter describes these multifactor experiments I ran in 2016, but is also unique because these experiments used only eggs from a single mother for each trial. Thus, I was able to examine both the interactions between warming and acidification and the variability of those impacts within a clutch and across the clutches of a breeding season. I find that responses within a clutch are relatively consistent, but means shift and variance increases with both warming and acidification. Across the season, I saw a number of different sensitivity patterns, with sensitivity to both warming and acidification increasing as the season went on. Warming appeared to be the more dominant stressor, however, and potentially swamped out acidification impacts by driving paralarvae to their minimal viable state. I find that warming was broadly antagonistic to acidification in all measured metrics. The data suggest that warming acts immediately and early in development, while acidification builds over time causing greater impacts to late development. I hypothesize that the antagonism I observe between these stressors is due, in large part, to warming decreasing development time, and thus exposure time to acidification.

The experiments and results described in this dissertation demonstrate the complexity of running stress experiments with a non-model organism, particularly one as plastic as a squid. Taking data from any individual experiment or even set of experiments within a year would not have provided a substantial or correct picture of how this life stage of this organism responds to 
acidification. I reran and adaptively shifted these experiments as needed to try to best capture the processes and variability of sensitivity being expressed by the squid. While I do find scenarios in which squid embryos and paralarvae are impacted by acidification, the dosage required is high and it appears to depend greatly on the state of the parents. Temperature seems to have a role in this parental conditioning, but also mitigates acidification impacts on the embryonic scale, further complicating our ability to predict impacts under future ocean change. This dissertation serves to highlight the importance of taking into account the life history of an organism as part of the experimental design of anthropogenic stress studies and the need for more robust, long-term, repeated, and generational studies of organismal response to stress. 


\section{Chapter 2}

\section{Dose-dependence and small-scale variability in responses to ocean acidification during squid, Doryteuthis pealeii, development}

This chapter was originally published as: Zakroff C, Mooney TA, Berumen ML (2019) Dose-dependence and smallscale variability in responses to ocean acidification during squid, Doryteuthis pealeii, development. Marine Biology 166:62. doi: 10.1007/s00227-019-3510-8. The Supplementary Materials for this chapter can be found in Appendix A. 


\begin{abstract}
Coastal squids lay their eggs on the benthos, leaving them to develop in a dynamic system that is undergoing rapid acidification due to human influence. Prior studies have broadly investigated the impacts of ocean acidification on embryonic squid, but have not addressed the thresholds at which these responses occur or their potential variability. We raised squid, Doryteuthis pealeii (captured in Vineyard Sound, Massachusetts, USA: $41^{\circ} 23.370 \mathrm{~N} 70^{\circ}$ $46.418^{\prime} \mathrm{W}$ ), eggs in three trials across the breeding season (May - September, 2013) in a total of six chronic $\mathrm{pCO}_{2}$ exposures $(400,550,850,1300,1900$, and $2200 \mathrm{ppm})$. Hatchlings were counted and subsampled for mantle length, yolk volume, hatching time, hatching success, and statolith morphology. New methods for analysis of statolith shape, rugosity, and surface degradation were developed and are presented (with code). Responses to acidification (e.g., reduced mantle lengths, delayed hatching, and smaller, more degraded statoliths) were evident at $\sim 1300$ ppm $\mathrm{CO}_{2}$. However, patterns of physiological response and energy management, based on comparisons of yolk consumption and growth, varied among trials. Interactions between $\mathrm{pCO}_{2}$ and hatching day indicated a potential influence of exposure time on responses, while interactions with culture vessel highlighted the substantive natural variability within a clutch of eggs. While this study is consistent with, and expands upon, previous findings of sensitivity of the early life stages to acidification, it also highlights the plasticity and potential for resilience in this population of squid.
\end{abstract}




\section{Introduction}

Addressing the potential effects of ocean acidification (OA) has become a major concern for the management of coastal ecosystems. This includes the northwest Atlantic coastal region where urban development and freshwater influx can exacerbate decreasing $\mathrm{pH}$ caused by anthropogenic carbon dioxide $\left(\mathrm{CO}_{2}\right)$ (Gledhill et al. 2015). This ecosystem is home to a suite of fisheries species that use nearshore habitats as breeding grounds. Early life stages are expected to be more sensitive to environmental stress than juveniles or adults, so rapidly intensifying impacts such as acidification are of particular concern (Byrne 2011; Haigh et al. 2015).

Loliginid squids, such as the Atlantic longfin squid, Doryteuthis pealeii, are common fixtures in many continental shelf ecosystems. These animals are important commercially, with D. pealeii supporting a major New England fishery with 18,000 mt landings in 2016 (NOAA Fisheries 2019). They are also a central support structure for the coastal food web, acting as both prey and predator throughout their life history (Jacobson 2005). During reproduction, adults affix their encapsulated offspring to the nearshore benthos and the young must develop under whatever conditions occur there, potentially resulting in chronic exposure to stressors such as acidification (Jacobson 2005; Fabry et al. 2008; Byrne 2011).

The Atlantic longfin squid comes inshore along the northwest Atlantic coastline from May - October to breed, producing clusters or "mops" of encapsulated embryos that are bound to benthic structure or substrate (Jacobson 2005). Egg laying habitat along the North American Atlantic shelf has been observed to occur at depths shallower than $50 \mathrm{~m}$ in salinities of 30-32 ppt and temperatures ranging from 10-23 ${ }^{\circ} \mathrm{C}$ (McMahon and Summers 1971; Jacobson 2005). Reported shelf carbonate system profiles across D. pealeii egg laying habitat suggest a potential exposure range of $8.2-7.88 \mathrm{pH}_{\mathrm{t}}$ during breeding season (250 - $600 \mathrm{ppm} \mathrm{CO}_{2}$; values calculated across depth/temperature extremes using $\mathrm{CO}_{2} \mathrm{SYS}$ with data from Wang et al. 2013). Whether $\mathrm{pH}$ or others oceanographic parameters, such as oxygenation, determine $D$. pealeii egg laying habitat has not been reported to our knowledge, but observations of the California market squid, Doryteuthis opalescens, demonstrate a preference for oxygen levels greater than $160 \mu \mathrm{mol}$ and $\mathrm{pH}_{\mathrm{t}}$ greater than 7.8 (Navarro et al. 2018).

The embryos are packaged inside an egg capsule comprised of mucosal proteins with several hundred siblings, all developing and respiring together (Arnold et al. 1974; Jacobson 2005). Under natural conditions, the inside of these capsules become increasingly anoxic and 
acidic as development proceeds, reaching pH values as low as 7.34 (Gutowska and Melzner 2009; Long et al. 2016). The only energy source available to these embryos for use in growth, development, and homeostasis is the yolk provided by the mother (Arnold et al. 1974; Steer et al. 2004). While cephalopods are adept at maintaining internal $\mathrm{pH}$ balance through the activation of proton secreting transporters within ion-transport epithelia, this process is energetically costly (Hu et al. 2010, 2013). Sensitivity to $\mathrm{pH}$, and the associated homeostatic costs, may vary depending on the cephalopod species and the developmental stage as well (Hu et al. 2010, 2011a).

Previous studies have looked broadly at the potential impacts of acidification on developing loliginid squid embryos. Embryos of Loligo vulgaris, removed from the egg capsule and exposed to acidification $\left(\mathrm{pCO}_{2} \sim 1650 \mathrm{ppm}\right)$ and warming $\left(+2^{\circ} \mathrm{C}\right)$, demonstrated delays in development as well as a dramatic decrease (47\%) in embryonic survival (Rosa et al. 2014a). Doryteuthis opalescens egg capsules cultured under decreased $\mathrm{pH}\left(\mathrm{pH} 7.57, \mathrm{pCO}_{2} \sim 1440 \mathrm{ppm}\right)$ and hypoxia ( $80 \mu \mathrm{M} \mathrm{O}_{2}$ ) also showed delays in embryogenesis (Navarro et al. 2016). Further, this study suggested that the combination of these stressors, potentially driven by the hypoxia, resulted in smaller embryonic statoliths, the aragonitic structures responsible for the squid's sensing of balance, orientation, and sound (Navarro et al. 2016). Kaplan et al. (2013) measured D. pealeii paralarvae hatching from eggs reared in high acidification (2200 ppm) and observed both a reduction of statolith size and apparent structural degradation, although the latter was only qualitatively defined. This study also noted a delay in development time and a reduction in paralarval dorsal mantle length as a result of the high acidification dose (Kaplan et al. 2013).

While it is becoming apparent that loliginid squid can be influenced by OA, the additional variables and limited $\mathrm{pCO}_{2}$ concentrations tested in some of the prior studies make it challenging to assess the scope of $\mathrm{pCO}_{2}$ impacts. To aid management of this key fisheries species, it is crucial to address whether developmental changes occur gradually with increasing OA or if there is some "tipping point" beyond which effects are significant. Studies addressing early life history are critical because these animals form the foundation for future populations and this phase of development may be particularly vulnerable (Byrne 2011). While documenting fundamental OA effects on these squid is necessary, it is also vital to move beyond basal observations of impacts to address how these animals might cope with this stressor, such as 
through management of the energy budget, and explore the potential for resiliency within a hatchling cohort.

The experiments performed here were designed to expand upon the work of Kaplan et al. (2013) in order to more thoroughly describe the sensitivity of $D$. pealeii to ocean acidification and understand the mechanisms by which it impacts the early development of this species. We reared $D$. pealeii eggs in a range of $\mathrm{pCO}_{2}$ treatments in order to examine dose-dependent responses under the hypothesis that between the ambient and 2200 ppm treatments used in the original study lie some physiological threshold for OA. Based on the results from Kaplan et al. (2013), we hypothesized that D. pealeii compensated for $\mathrm{pH}$ stress by slowing development rate and reducing energy spent on growth, however we did not have a sufficiently robust picture of energy physiology to support this idea. We therefore expanded upon the previous analyses of dorsal mantle length, hatching time, and statolith size and quality (quantifiable metrics were developed), and added measurements of yolk volume (to quantify potential energy consumption effects) and hatching success (to address embryonic survival). We also analyzed data at a high resolution, across multiple hatching days in repeated trials over the breeding season, and describe the natural variability, the potential for resiliency, observed in the squid eggs in response to chronic acidification stress.

\section{Materials and Methods}

\section{Squid collection and husbandry}

Experiments were performed at the Woods Hole Oceanographic Institution

Environmental Systems Laboratory (ESL), Woods Hole, Massachusetts, USA from June through August of 2013. Peak breeding season for D. pealeii, in this region, when the squid move into the nearshore of New England, typically falls between May and September (Arnold et al. 1974; Jacobson 2005). Squid were captured in Vineyard Sound by trawls performed by the Marine Biological Laboratory (MBL) in 10-30 meters water depth at the Menemsha Bight of Martha's Vineyard, a locally known breeding ground. Adult squid were hand-selected directly from the trawl ship at the dock. Eighteen medium-sized individuals (20-25 cm dorsal mantle length) that did not appear stressed (those calmly hovering or resting at bottom of the holding tank) or damaged (those without fin tears or skin lesions) were carefully transferred to seawater-filled coolers and driven to the ESL. On top of condition, reproductively active females were selected 
for based on their bright orange accessory nidamental gland, while males with dense sperm packets visible in the posterior mantle were chosen. Transport occurred as immediately $(<6$ hours post-capture), expediently, and gently as possible to minimize stress.

At the ESL, squid were transferred from the coolers into two flow-through cylindrical holding tanks (120 cm diameter, $70 \mathrm{~cm}$ depth) fed with water pumped directly from Vineyard Sound to the ESL and continuously bubbled with air. Squid were selected and housed in a 2:1 female to male ratio in order to increase the probability of breeding and egg deposition. Ambient Vineyard Sound seawater was sand-filtered and cooled to $15^{\circ} \mathrm{C}\left(\right.$ Salinity $\left.=33 \mathrm{psu}, \mathrm{pH}_{\mathrm{nbs}}=7.96\right)$. This temperature falls within the range experienced naturally during the breeding season, but below peak summer temperatures for Vineyard Sound $\left(10.2\right.$ - 25.8 ${ }^{\circ} \mathrm{C}$ from May - October 2013 from NOAA Station BZBM3). Compared to maintaining squid at ambient temperatures, maintaining squid at $15{ }^{\circ} \mathrm{C}$ served to reduce metabolic stress and the occurrence of infighting and cannibalism among the squid, which substantially increased the likelihood of successful egg production. Squid were fed killifish, Fundulus heteroclitus, caught in local saltwater ponds once to twice per day, depending on demand. All squid were fed and managed in the ESL until they died after breeding. New adult squid were acquired for each trial.

Female squid laid eggs two to three days after being brought to the ESL. The egg capsules of this species of squid are long, orange-tinged fingers housing 90 - 300 eggs, which are tied together with mucosal proteins into mops that are bound to benthic substrate or structures (Arnold et al. 1974; Maxwell and Hanlon 2000). In the morning, tanks were examined and if egg capsules were discovered they were immediately hand-transferred into a bucket of seawater from the adult tank and carried into the room with the acidification and culture system. Egg capsules of good quality (thin, oblong, tinted orange, and undamaged) were randomly hand-sorted into the experimental culture cups, two egg capsules per culture cup, to initiate a trial (described below).

\section{Ocean acidification system}

Seawater was acidified in a flow-through culture system constructed in a separated room within the ESL. Vineyard Sound seawater pumped into the ESL went through the facility's sandfilters and was then subsequently heated to $20^{\circ} \mathrm{C}$. This temperature represents the average sea surface temperature for Vineyard Sound over the breeding season $\left(19.5^{\circ} \mathrm{C}\right.$ from May - October 2013 from NOAA Station BZBM3) and resulted in a consistent fourteen day development period 
for the squid embryos under control conditions. The heated seawater then went through an additional $10 \mu \mathrm{m}$ filter (Hayward FLV Series, $10 \mu \mathrm{m}$ felt bag, Hayward Industries, Inc., Rockville, Maryland, USA) to limit small zooplankton, particulate matter, and algae. The water was further treated with a UV sterilizer (Emperor Aquatics Smart HO UV Sterilizer, Model 025150, Pentair Aquatic Eco-Systems, Inc., Cary, North Carolina, USA), in order to reduce harmful protozoa, although flow rate was too high for the seawater to be completely sterilized of microorganisms.

The resultant cleaned and heated water was then output into the header tank of the acidification system, which was vigorously bubbled with compressed air. Between the filtration and heating systems of the ESL and this system header tank, it is expected that most input seawater is mixed over the course of several hours and is not subject to small-scale environmental variability, however fluctuations, particularly of alkalinity, were possible. Fine temporal scale water quality measurements were not performed. Water flowed out of the header into four H-shaped PVC gas equilibration chambers (Figure S1).. Two air stones in each leg of the ' $\mathrm{H}$ ' of an equilibration chamber bubbled the flowing seawater with the treatment mixture of compressed air and $\mathrm{CO}_{2}$. During the first two trials in July (Jul 3 \& Jul 11; Table 1) it was discovered that the ambient seawater in the ESL had an elevated concentration of equilibrated $\mathrm{CO}_{2}: 550 \mathrm{ppm}$ in the facility compared to $400 \mathrm{ppm}$ for seawater samples taken at depth at the pump intake in Vineyard Sound (carbonate system measurements analyzed with VINDTA). Subsequently, the ambient treatment line of the equilibration chamber section of the acidification system was rebuilt to include two additional chambers, resulting in a line wherein the water was first degassed by $\mathrm{N}_{2}$ before being re-equilibrated with ambient compressed air in the following two chambers.

Gas mixtures were produced by combining compressed air, introduced at 30 psi from an air compressor within the ESL, with cylinder $\mathrm{CO}_{2}$. The compressed air was split using a six-way manifold in order to provide aeration through the air stones in the header tank and the equilibration chambers, feed the manifold providing gas to the control culture cups, as well as feed three mass flow controllers (GFC17, Aalborg, Orangeburg, New York, USA), which brought the flow rate down to $4.51 \mathrm{~min}^{-1}$. Carbon dioxide was also delivered at $30 \mathrm{psi}$ to a parallel set of three mass flow controllers (GFC37, Aalborg, Orangeburg, New York, USA), which were adjusted in order to produce the desired concentrations of $\mathrm{CO}_{2}$. The air and $\mathrm{CO}_{2}$ 
lines were joined downstream of the mass flow controllers and these mixtures were then fed into manifolds which split the gas between the air stones in the equilibration chambers and the bubblers in the culture cups of each treatment. A $\mathrm{CO}_{2}$ analyzer (model s151, Qubit Systems, Kingston, Ontario, CA), 3-point calibrated with three reference gases (cylinders with 0, 362, and 1036 ppm $\mathrm{CO}_{2}$, Corp Brothers, Inc., Providence, Rhode Island, USA), was used to check $\mathrm{CO}_{2}$ concentration in the gas mixtures prior to each trial.

Treatment water flowed from the equilibration chambers into four PVC manifolds from which individual drip lines were connected to the individual culture cups. Egg capsule culture cups were constructed from 1-liter PET food service containers (Solo Foodservice, Lake Forest, IL), which had been pre-soaked in seawater for at least 24 hours and cleaned with deionized water to remove any residues or toxins. These cups had a small rectangular outflow window $(2 \mathrm{x}$ $4 \mathrm{~cm}$ ) cut high on the side and screened with $5 \mu \mathrm{m}$ mesh, which retained the hatched paralarvae. Each cup was sealed with a lid pierced with two holes, one for the treatment drip line and one for a gas line to bubble in the treatment gas mixture (Figure S1). Drip lines were fed to the bottom of the culture cup to ensure mixture and overturn and prevent waste accumulation. Treatment water inflow was maintained at a rate of approximately $20 \mathrm{~L}^{\text {day }}{ }^{-1}$ in each cup, which allowed for sufficient time for the water to equilibrate within the H-shaped chambers. The bubbling line was placed approximately midway under the screened outflow window in order to circulate the water without disturbing the egg capsules while also pushing resulting hatchlings away from the screen. Water from the culture cups outflowed into a communal water bath maintained at $20{ }^{\circ} \mathrm{C}$ using both aquarium chillers (Oceanic Aquarium Chiller 1/10hp, Oceanic Systems, Walnut Creek, California, USA) and a set of controllable aquarium heaters (JÄGER 3603, EHEIM GmbH and Co., Deizisau, DE).

The system consisted of two water baths, allowing for two staggered trials to be run simultaneously (Figure S1).. Each water bath housed three acidification treatments with four culture cups each for a total of twelve cups. Three cups per treatment were used to culture egg capsules, while the fourth was used as an abiotic control to monitor water chemistry. An Onset HOBO data logger (HOBO pendant model UA-004-64, Onset Data Loggers, Bourne, Massachusetts, USA) was placed in each water bath to monitor temperature and ambient light; recordings were taken every 15 minutes. Water bath 1 had a mean temperature of $20.49 \pm 0.69$ ${ }^{\circ} \mathrm{C}$ (mean \pm standard deviation) and water bath 2 had a mean temperature of $20.26 \pm 0.49{ }^{\circ} \mathrm{C}$ 
across experiments (Table 1). The egg capsules did not undergo temperature acclimation during the transfer from the $15^{\circ} \mathrm{C}$ holding tank to the $20{ }^{\circ} \mathrm{C}$ culture cup, as this level of temperature shift at this early stage of development was not seen to impact embryonic development or survival, or the morphology and physiology of the paralarvae, within the metrics measured here. Ceiling mounted fluorescent lighting in the ESL room containing the culture system was set to a 14:10 light:dark photoperiod to reflect the average natural light cycle for the region. The system was allowed to run for several days prior to acquiring squid in order to ensure equilibration of $\mathrm{CO}_{2}$ and temperature and balancing of gas bubbling and water flow.

The $\mathrm{pH}_{\mathrm{nbs}}$ of all of the culture cups, both with and without egg capsules, was monitored by taking samples every three days and measuring using a pH probe (Orion Star ${ }^{\text {TM }}$ A329, Thermo Fisher Scientific Inc., Waltham, Massachusetts, USA). These measurements were not considered an accurate proxy for seawater $\mathrm{pCO}_{2}$, but were used to regularly check $\mathrm{pH}$ stability within the system. Respiration of the egg capsules did not notably change $\mathrm{pH}$ of the experimental cups compared to the procedural controls. On the day a trial began, and every seven days after (twice more overall), water samples from the fourth cups, the abiotic controls, were taken for high precision carbonate chemistry measurements. $\mathrm{pH}_{\mathrm{t}}$, salinity, and alkalinity data were recorded following the methods adapted from White et al. (2013). In brief, $\mathrm{pH}_{\mathrm{t}}$ was recorded using $2 \mathrm{mM} m$-cresol purple indicator dye in a spectrophotometer (USB4000 Spectrometer, Ocean Optics, Dunedin, Florida, USA) using methodology adapted from Clayton and Byrne (1993) and Dickson et al. (2007). Parallel to the $\mathrm{pH}_{\mathrm{t}}$ readings, $120 \mathrm{~mL}$ glass bottle samples were taken for salinity measurement, which were later analyzed using a Guildline model 8400B “Autosal” laboratory salinometer (Guildline Instruments, Smith Falls, Ontario, Canada). For total alkalinity, $20 \mathrm{~mL}$ acid-washed, glass scintillation vials were filled with treatment seawater and poisoned with $10 \mu \mathrm{L}$ saturated mercuric chloride before being sealed for later analysis. One $\mathrm{mL}$ subsamples were processed in duplicate on an automated small volume titrator (Titrando 808, Metrohm AG, Herisau, $\mathrm{CH}$ ) programmed to run Gran titrations with $0.01 \mathrm{~N} \mathrm{HCl}$. ESL seawater samples of known alkalinity, measured using a VINDTA (marianda, Kiel, DE), were used as calibrating standards. If duplicate sets had a difference between samples of $4 \mu \mathrm{mol} \mathrm{\textrm {kg } ^ { - 1 }} \mathrm{seawater}^{-}$ or greater, a second duplicate set was run and the average of the four values was used. The high precision carbonate chemistry measurements $\left(\mathrm{pH}_{\mathrm{t}}\right.$, salinity, and total alkalinity), as well as the water bath temperature readings, were input into CO2SYS (Pierrot et al. 2006). Dissociation 
constants from Mehrbach et al. (1973) and sulfate constants from Dickson (1990) were used in order to calculate $\mathrm{pCO}_{2}$ and aragonite saturation state $\left(\Omega_{\text {arag }}\right)$ for the equilibrated seawater of each acidification treatment (Table 1).

\section{Experimental trials and paralarval sampling}

Trials were initiated by the morning discovery of a mop of egg capsules in the adult holding tanks and are referred to by this laying date throughout this analysis (Table 1). Egg capsules were immediately transferred by seawater bucket(s) to the culture/acidification system room. There they were randomly hand-sorted into the culture cups of an available water bath, two egg capsules per experimental cup (eighteen total egg capsules per trial). Because D. pealeii females store sperm and often mate with multiple males, and given that multiple females will lay egg capsules together in the same mop of eggs, the egg capsules used here are of distinctly complex and unknown parentage (Hanlon and Messenger 1998; Buresch et al. 2009). Thus, measurements of adult squid size and weight were not taken. At most, these egg capsules can be considered to represent a haphazard (since size and condition were considered during selection from the trawl catch) sampling of the Vineyard Sound population at a particular point in time during the breeding season. The August trial (Aug 7), however, was initiated by the discovery of egg mops in both of the adult squid holding tanks on the same morning. As a result, egg capsules were randomly distributed, but into a specific set of cups, such that the first cup of each treatment contained two egg capsules from holding tank A, the second cup contained two egg capsules from holding tank B, and the third cup contained one capsule from each tank. Discrete separation of egg capsule parentage therefore occurred for this trial (Cup 1: AA, Cup 2: BB, Cup 3: $\mathrm{AB})$; as much as is possible excepting the probability that a female from one tank had stored the sperm of the male of another tank while in the wild or during capture.

A total of three trials were performed using six carbon dioxide concentrations between 400 ppm and 2200 ppm (Table 1; 400, 550/ESL Ambient, 850, 1300, 1900, and 2200 ppm). The Jul 3 trial was designed to repeat the levels used in Kaplan et al. (2013), atmospheric ambient and 2200 ppm, and add a 1300 ppm midpoint. As stated above, the discovery of the elevated $\mathrm{pCO}_{2}$ in the ESL seawater affected the ambient treatment of this first trial, and so is reported as ESL Ambient (550) rather than an atmospheric concentration control. Vertical profiles and water column bottle samples taken in the Menemsha Bight in Vineyard Sound in 2014 and 2015 
indicate a consistently well-mixed system with near atmospheric $\mathrm{CO}_{2}$ concentrations from which this ESL Ambient deviates, but not greatly (July - September 2014, mean bottom [20 m] pCO of $^{2}$ $471.8 \mathrm{ppm}$ and average surface to bottom difference of $2.2 \mathrm{ppm}$; May - September 2015, mean bottom $\mathrm{pCO}_{2}$ of $484.9 \mathrm{ppm}$ and average surface to bottom difference of $12.7 \mathrm{ppm}$; Zakroff \& Mooney, unpublished data). The Jul 11 trial was intended to be run with ambient control, 850 ppm, the midpoint between 400 and 1300, and 2200 ppm. It was instead run without an ambient control as that treatment line was under reconstruction, and the still active $1300 \mathrm{ppm}$ line was included in its place. Two trials were planned to follow the reconstruction of the $400 \mathrm{ppm}$ line, one using 1600 ppm and one using 1900 ppm, in order to evenly fill the space between 1300 and $2200 \mathrm{ppm}$. The trial incorporating the $1600 \mathrm{ppm} \mathrm{CO}_{2}$ treatment was lost, however, due to a failure of the compressed air system resulting in extended exposure of the egg capsules to degassed, and thus deoxygenated, water and is not reported here. The Aug 7 trial was run successfully with the appropriate $400 \mathrm{ppm} \mathrm{CO}_{2}$ control, representing present atmospheric concentrations, in place, the $2200 \mathrm{ppm}$ treatment acting as the consistent concentration measured across all trials, and the 1900 ppm treatment as planned.

The squid egg capsules were monitored, but otherwise left to develop undisturbed in their culture cups within the acidification system. At eleven to twelve days into development, morning checks for premature hatching began. Hatching typically initiated in the ambient treatment thirteen to fourteen days into development, as expected. Once hatching began, paralarvae were subsampled for a range of experiments. The results of the developmental and morphometric analyses, described below, are reported here, while concurrent behavioral work that subsampled paralarvae from these same experiments has been reported separately (Zakroff et al. 2018).

\section{Dorsal mantle length}

Ten paralarvae (fewer if fewer were available) were subsampled from each cup of each treatment each day for the first four to six days of hatching (dependent on hatching dynamics) to be photographed for dorsal mantle length (DML) measurement. When counting out the animals, individuals were pipetted from their culture cup into their own wells within a 24-well plate (Falcon ${ }^{\circledR}$ Brand $2.0 \mathrm{~cm}^{2}$ well area, $3.5 \mathrm{~mL}$ well volume, Corning Inc., Corning, New York, USA). Wells were filled with the appropriate treatment seawater with a few drops of $7.5 \% \mathrm{w} / \mathrm{v}$ $\mathrm{MgCl}_{2}$ mixed with equal part seawater added as an anesthetic. Individuals were then carefully 
pipetted into a drop of treatment seawater on a watch glass and placed under a dissecting scope (SteREO Discovery.V8, Carl Zeiss AG, Oberkochen, DE). Once a paralarva was confirmed to be oriented with the dorsal surface up, most easily recognized on D. pealeii by the hexagonal pattern of chromatophores on the dorsal surface of the head, an attached camera (G12, Canon USA, Melville, New York, USA) was used to take a photograph (Figure 1A). Prior to taking sample photographs, the dissecting scope was focused using the first paralarva and then a calibrating photograph of a millimeter ruler was taken for that day of data collection. If for any reason the focus needed to be changed or photography was interrupted and the camera had to be reset, a new calibration photo was taken. No premature paralarvae, those with remnant external yolk present, nor any that had damage to the mantle, were included in the DML photography dataset. The images of the subsampled paralarvae were later measured for DML using ImageJ (National Institutes of Health, Rockville, Maryland, USA).

\section{Yolk sac volume}

An additional 10 paralarvae (fewer if fewer were available) from each cup of each treatment were subsampled each day for lipid staining and preservation using methods adapted from Gallager et al. (1986). In brief, subsampled paralarvae were pooled by treatment cup in a 24-well plate and then euthanized with an increasing dose of $7.5 \% \mathrm{w} / \mathrm{v} \mathrm{MgCl}_{2}$ mixed with equal part seawater. The paralarvae were then quickly fixed with $10 \%$ formalin in order to prevent contraction of the mantle during staining and preservation. The seawater containing the $\mathrm{MgCl}_{2}$ and formalin was then pipetted off and the fixed paralarvae were submersed in oil red $\mathrm{O}$ suspended in ethylene glycol, covered, and left to stain overnight. The subsequent morning, the stain was pipetted off and the paralarvae underwent two 30-min soaks in ethylene glycol to remove excess stain before being stored in ethylene glycol in labeled microcentrifuge tubes ( 0.65 $\mathrm{mL}$ Costar microcentrifuge tubes, Corning, Inc., Corning, New York, USA). No notable shrinkage as a result of euthanasia, brief formalin fixation, lipid stain, or ethylene glycol storage was observed, however this was not robustly measured, so analyses are reported under the assumption of either no shrinkage or consistent shrinkage across samples.

Oil red $\mathrm{O}$ effectively stained the interior yolk sacs, making them much more visible through the translucent mantle. Lipid-stained paralarvae were photographed in daily sets as described for the DML subset above, except that paralarvae were oriented either dorsal or ventral 
dependent on which produced the clearer image of the yolk sacs (preferentially ventral, but occasionally dorsal as in Figure 1B). The photos were processed in Image J following the methods of Vidal et al. (2002). In brief, lines were drawn measuring the length and width of both the anterior and posterior yolk sacs (Figure 1B). These values were input into formulas representing a three-dimensional shape approximating the volume of the yolk sac: a cone or cylinder for the anterior, and a rotational ellipsoid for the posterior. These results were then summed to get the total yolk volume (YV) for each individual paralarva.

\section{Hatching time and success}

Following all morphometric and behavioral work, the remaining paralarvae were counted as they were pipetted from a treatment cup into a petri dish containing water of the same

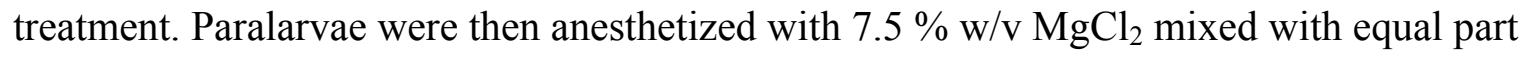
seawater and preserved in $97 \%$ ethanol in microcentrifuge tubes $(0.65 \mathrm{~mL}$ and $1.7 \mathrm{~mL}$ Costar microcentrifuge tubes, Corning, Inc., Corning, New York, USA) by treatment cup (the anesthetized DML subsamples were added back to their appropriate tube in preservation). Thus, no hatched animals remained, nor were any returned to their treatment cups at the end of each experiment day, and all paralarvae analyzed were from their day of hatching (less than 24 hours old). The total number of hatched paralarvae was summed at the end of hatching and used to calculate percent hatching in each treatment cup per day.

Hatching was considered finished in a treatment cup following two mornings with no newly hatched paralarvae. The two egg capsules within the treatment cup would then be removed, photographed, and dissected underneath a dissecting scope. Unhatched embryos were sorted and counted according to their stage of development, adapted from Arnold et al. (1974): early (stages 1 - 16), middle (stages 17 - 26), and late (stages 27 - 30). The total number of unhatched embryos was summed with the total number of hatched paralarvae to get the original count of embryos in each cup. The ratio of hatched paralarvae was compared with the total number of embryos to examine hatching success within each treatment cup.

\section{Statolith morphometrics}

Ethanol preserved paralarvae were dissected for their statoliths. An individual paralarva was placed on a glass cover slip underneath a dissecting scope and kept moist with $97 \%$ ethanol. 
Once separated, an individual statolith was rinsed with 97\% ethanol and all visible adhering tissue was removed. The statolith was then transferred onto a sticky carbon pad (C249/N $12 \mathrm{~mm}$ diameter self-adhesive carbon disc, TAAB Laboratories Equipment Ltd., Berks, England, UK) on a scanning electron microscopy (SEM) stub (12.7mm diameter aluminum mount, Electron Microscopy Sciences, Hatfield, Pennsylvania, USA). Only one, randomly chosen statolith from each individual paralarva was mounted for SEM imaging and approximately five statoliths (more if possible) per treatment cup (approximately fifteen statoliths per acidification treatment) were assessed. The SEM stubs were taken to the MBL Central Microscopy Facility where they were sputter-coated with $10 \mathrm{~nm}$ platinum and imaged using a Zeiss NTS Supra 40VP (Carl Zeiss AG, Oberkochen, DE).

SEM images (1024 x 768 px, TIFF files) were resized such that all statoliths were set to the same $6 \mathrm{px} \mu \mathrm{m}^{-1}$ scale (approximately $672 \mathrm{X}$ magnification) using Adobe Photoshop (Adobe Photoshop CC 2017, Adobe Systems Inc., San Jose, California, USA). The Photoshop quick selection tool was then used to select the statolith, carefully maintaining edge integrity. The selection was then cut to a new layer and that layer was saved separately as a PNG for the MATLAB surface analysis described below. The process was then backed up to the selection step and the selection was flood-filled black and again cut to a new layer. Statoliths were then reoriented such that the longest axis of the statolith was parallel to the horizontal axis of the image, the dome, the wider, lobe-like part of the structure, was placed to the right of the image and the rostrum, the thinner, wing-like projection, was to the left (note in Figure 2B, D that processing in Momocs, described below, flipped these so that the outline had the dome oriented left). For degraded or misshapen statoliths, a best approximation was used, with the longest axis being set horizontal and the subsequently wider side set to the right. The background layer was then flood-filled white to create a black silhouette of a statolith on a white background. These silhouetted statolith images were saved as JPEG files and compiled with the rest of the samples for import into the R (version 3.3.3, R Foundation for Statistical Computing, Vienna, AT) morphometrics package Momocs (Bonhomme et al. 2013) in RStudio (version 1.0.136, RStudio, Inc., Boston, Massachusetts, USA). Momocs took the silhouetted images and translated them into objects describing the outlines of the 2D shapes. Morphometric analysis of the outlines provided statolith length, width, surface area, rectangularity, and circularity. 
Two metrics were developed in order to quantitatively describe and compare qualitative observations of statolith degradation. The first was intended to describe the 'rugosity' of the statolith edge, e.g. whether the statolith had a smooth perimeter (Figure 2A) or a rough one (Figure 2C). Momocs describes a 2D shape via a series of points that demarcate its outline. Through extensive testing with a test set of shapes (described in the Supplementary Materials), it was found that calculating the variation of the internal angles between points on an outline at a resolution of 150 points resulted in the best description of shape outline complexity or 'rugosity.' Internal angle variance was calculated for all statolith outlines at this resolution using the Momocs objects in R (code available in the Supplementary Materials and at https://github.com/czakroff/Statoliths).

The second metric was intended to quantify the consistency of the visible statolith surface in the SEM image, e.g. whether a statolith had a smooth surface with organized calcium carbonate crystals (Figure 2A) or had a rough surface due to increased porosity or disorganized crystals (Figure 2C). This was achieved by analyzing the average variance of the pixel intensities in five boxes haphazardly placed on the statolith image. The scaled cutout statolith PNG images described above were loaded into a custom MATLAB (version R2016b, Mathworks, Inc., Natick, Massachusetts, USA) script (available in the Supplementary Materials and at https://github.com/czakroff/Statoliths). The script requires the user to click to mark the centroids of five $100 \times 100 \mathrm{px}$ squares (equivalent to $277.78 \mu \mathrm{m}^{2}$ of the statolith surface at this scale) that are placed on the statolith image (Figure 2A, C). The user can then iterate through this process to adjust the squares to ensure they are placed appropriately. Squares were placed in order to achieve as even a distribution over the statolith surface as possible while trying to avoid surface occlusions (salt crystals or remnant tissue), dramatic lighting gradients, and large cracks or breaks. The pixel variance of each box was calculated and then the average surface pixel variance over the five boxes was compiled for all sample statoliths.

\section{Statistics}

All statistical analyses were performed in Python (version 3.5.2, Python Software Foundation) using Jupyter Notebooks (Project Jupyter). All data, at all levels (trial, treatment, date, and cup), was tested for normality using Shapiro-Wilk tests $(\alpha=0.05)$ and through visual assessment of quantile plots and histograms. Differences in data that were normally distributed 
were tested with multi-factor Type II ANOVAs. Type II ANOVAs were selected in order to test factors independently, without ordering, and to not test a main effect in light of its interactions; the hypotheses tested are therefore if a factor in all of its forms has an impact on the dependent variable (Langsrud 2003). Under this framework, the presence of an interaction is of greater interest than a main effect. Treatment $\left(\mathrm{pCO}_{2}\right)$ and date were considered independent factors nested under trial, while cup was considered nested under $\mathrm{pCO}_{2}$. Significant $(P<0.05)$ results were further analyzed using a Tukey's HSD posthoc test. Statistics of normally distributed data are reported as means \pm standard deviation. Yolk sac volume data was log transformed to stabilize variance and then tested as with other parametric data; yolk volume data is reported as the mean and values \pm one standard deviation back transformed.

Nonparametric data were assessed for differences using a Kruskal-Wallis test (KW); significant $(P<0.05)$ results were further analyzed using a Dunn's posthoc test. Nonparametric statistics are reported as medians and interquartile range. Distributions of hatching and embryo counts were compared using G-tests. Scatterplots of data by trial are presented with trend lines, primarily as a visual aid. These lines were assessed by linear,regression (LR), significance $(\alpha=$ 0.05), but as they were run on three data points we are not suggesting they are statistically powerful. Data compiled across trials was corrected for trial variability (likely due to variability from season/cohort/parentage) by taking the differences between samples and the trial mean, allowing for a comparison of effect size/response slopes (see note on assumptions below). Compiled data were assessed by piecewise linear regression (minimum of three data points per

regression) and the model with the best fit (highest $\mathrm{R}^{2}$ ) is presented. Piecewise regressions were tested for significant difference against the null hypothesis model of two lines, with different means, each with a slope of zero using a parametric bootstrap.

\section{Results}

\section{Water quality}

No significant differences in $\mathrm{pH}_{\mathrm{t}}$ or calculated $\mathrm{pCO}_{2}$ were found between cups of the same treatment or across time in the fourth culture cup $\left(\mathrm{KW}, P>0.05\right.$ for $\mathrm{pH}_{\mathrm{t}}$ and $\mathrm{pCO} 2$ for all treatments of all trials). Input gas mixtures of carbon dioxide and air were consistent throughout the experiment, resulting in consistent $\mathrm{pH}_{\mathrm{t}}$ values (Table 1). Salinity and temperature also remained constant across a trial, but was slightly more variable depending on the cycling routine 
and sensitivity of the control chiller (Table 1). Temperature also shifted slightly across trials, likely as an effect of changing local environmental temperatures. Calculated aqueous $\mathrm{pCO}_{2}$ concentrations varied slightly from input gas concentrations, typically being slightly higher, which could be a result of variability in ESL water alkalinity, inconsistency in the flow rate and equilibration rate of treatment water, or a combination of these factors. Equilibrated $\mathrm{pCO}_{2}$ variability increased with higher input concentrations, with the strongest acidification treatments being the hardest to maintain a consistent equilibration in. This increased variability is likely a result of choosing a flow-through egg capsule culture system rather than using a closed or recirculating system. Results are analyzed and reported grouped by input gas concentration rather than calculated aqueous $\mathrm{pCO}_{2}$ concentrations for concision and clarity.

\section{A note on assumptions}

There was significant trial-to-trial variability in the response of these developing squid to ocean acidification stress. Data analysis and figures presented examine data both by individual trial and compiled across trials. We therefore sought to clarify assumptions being made in the analysis and compilation of these data. Due to challenges imposed by the facility, the Jul 11 trial did not have an ambient $\mathrm{pCO}_{2}$ control. Based on the results across metrics, the similarities between the Jul 3 ESL Ambient and the Jul 11850 ppm data (effect sizes in mantle length and hatching time between these levels and $2200 \mathrm{ppm}$, in particular), we chose to include these data in compiled graphics and analyses.

Dorsal mantle length compared across lowest $\mathrm{pCO}_{2}$ treatments of each trial showed no difference between Jul 3 ESL Ambient and Jul 11850 ppm, but both had significantly larger paralarvae than the Aug 7400 ppm clutch (ANOVA, F $(2,492)=9.874, \mathrm{P}<0.001$; Tukey, $\mathrm{P}<$ 0.05; values reported below). Yolk volume, however, showed no difference between the Jul 11 $850 \mathrm{ppm}$ and the Aug $7400 \mathrm{ppm}$ while both were significantly reduced compared to the Jul 3 ESL Ambient (ANOVA, F $(2,471)=155.3, \mathrm{P}<<0.001$; Tukey, $\mathrm{P}<0.05$; values reported below). These shifting baselines, a consequence of seasonal, cohort, and/or maternal effects,

must be kept in mind when examining the compiled data for a more generalized population response to acidification.

\section{A note on egg number}


During manuscript preparation, a reviewer suggested that the relationship between measured factors (primarily DML and YV) and number of eggs in the egg capsules could be examined for potential trade-offs in maternal investment. As two egg capsules were used per cup, we could at best calculate average egg number for each treatment cup ([\# hatchlings + \# unhatched eggs] / 2). Rather than showing a trade-off, the data suggested a potential increase in both metrics with increasing number of eggs, although the correlation is much stronger for DML (Figure S4). While a relationship between initial egg size and hatchling DML has been described (Laptikhovsky et al. 2018), as well as negative correlations with egg density (removed eggs in petri dishes; Villanueva et al. 2011), a positive correlation between number of eggs and DML or YV has not been reported for multi-egg per egg capsule squids to our knowledge.

Our data represent three egg clutches laid by unknown parents (preselected for size/condition) taken in one breeding season and is not robust enough to consider reevaluating the entire dataset by (particularly without literature support). Type II ANOVAs of DML and YV run with only egg number as an independent covariate and cup (numbered individually rather than nested in treatment) demonstrated no effect of cup ( $\mathrm{P}>>0.05)$ and a substantial effect of egg number $(\mathrm{P}<<0.001)$ and a substantial interaction between egg number and cup $(\mathrm{P}<<0.001)$ in both metrics across trials (Table S5) suggesting that within the scope of our statistical models, these factors represent the same effect (if egg number were categorical, it and cup would be indistinguishable statistically). Statistical models incorporating egg number as an independent continuous covariate are reported in the Supplementary Materials (Tables S7 \& S8), but most statistics and data presented here are done so under the assumption that random selection of egg capsules accounted for this potential source of variability.

\section{Dorsal mantle length}

Dorsal mantle length decreased with increasing $\mathrm{pCO}_{2}$ in all trials (Figure 3). Overall compiled data showed a significant effect of trial and cup on DML, with near significant effects of $\mathrm{pCO}_{2}$ and the interaction between $\mathrm{pCO}_{2}$ and hatching date (Table 2). Paralarvae in the Jul 3 trial showed a broadly linear, but non-significant, decrease of DML with increasing $\mathrm{pCO}_{2}$ (LR, $P$ $=0.106$ ). Each treatment in this trial was significantly different from the others (Table S1; Tukey, $P<0.05$ : ESL Ambient/550: $1.64 \pm 0.11 \mathrm{~mm} ; 1300$ ppm: $1.59 \pm 0.12 \mathrm{~mm} ; 2200$ ppm: $1.56 \pm 0.12 \mathrm{~mm}$ ). The Jul 11 trial also showed a significant decrease in DML with increasing 
$\mathrm{pCO}_{2}$ (Table $\left.\mathrm{S} 1\right)$, but showed a non-linear (LR, $\left.P=0.206\right)$ step-wise response, with the $850 \mathrm{ppm}$ $(1.63 \pm 0.12 \mathrm{~mm})$ and $1300 \mathrm{ppm}(1.63 \pm 0.11 \mathrm{~mm})$ treatments being grouped (Tukey, $P<0.05)$ separately from the $2200 \mathrm{ppm}$ treatment $(1.53 \pm 0.12 \mathrm{~mm})$. Paralarvae in the Aug $7 \mathrm{clutch}$ demonstrated a weaker, but significant reduction in DML with increased acidification (Table $\mathrm{S} 1)$. Again, a step-wise (LR, $P=0.123)$ response was seen, with the $400 \mathrm{ppm}$ treatment $(1.59 \pm$ $0.13 \mathrm{~mm}$ ) having significantly (Tukey, $P<0.05)$ larger paralarvae than both the $1900 \mathrm{ppm}(1.53$ $\pm 0.13 \mathrm{~mm})$ and $2200 \mathrm{ppm}(1.53 \pm 0.12 \mathrm{~mm})$ treatments. Compiled by difference from trial mean, piecewise regression indicated a two line model, of a low $\mathrm{pCO}_{2} /$ greater DML group and a higher $\mathrm{pCO}_{2} /$ smaller DML group with breakpoint at $1300 \mathrm{ppm}$, best fit $\left(\mathrm{R}^{2}=0.858\right)$ the data and was not significantly different from the stepwise null hypothesis $(P=0.363$, Figure 3$)$. The compiled dataset of differences showed a significant relative decrease in DML with increased acidification (ANOVA, $\mathrm{F}_{8,1440}=16.50, P<0.001$ ), with statistical groupings splitting at the 1300 ppm treatment (Tukey, $P<0.05$; Figure 3).

The significant interaction between $\mathrm{pCO}_{2}$, hatching date, and cup on DML in the Jul 3 trial had the greatest effect size ( $\Omega^{2}$; Table 2). Interactions between $\mathrm{pCO}_{2}$ and hatching date alone were also significant (Table 2). The ESL Ambient/550 ppm treatment showed no difference in mean DML across the hatching days (ANOVA $P>0.05$; Table S2), despite a significantly increasing trend (LR, $P=0.023$; Figure 4). The $1300 \mathrm{ppm}$ samples were more variable, with significant differences in DML over the days of hatching (Table S2), but no corresponding trend (LR, $P=0.780$; Figure 4). The $2200 \mathrm{ppm}$ exposure approached significance for both mean DML over time and a slight decreasing trend (Table S2; LR, $P=0.090$; Figure 4). The effect of cup (nested in treatment) was significant, with a similar effect size to the interaction of $\mathrm{pCO}_{2}$ and date (Table 2). Details of responses by cup have been placed in the Supplementary Materials for manuscript brevity, except for the Aug 7 trial.

The Jul 11 trial also showed a significant interaction of $\mathrm{pCO}_{2}$, hatching date, and cup on DML (Table 2). While all factors and interactions showed significant effects on DML, date and the interaction between $\mathrm{pCO}_{2}$ and hatching date had the greatest effect sizes (Table 2). All three $\mathrm{pCO}_{2}$ treatments showed significant effects of hatching date on DML (Table S2). While all treatments showed decreasing paralarvae size with time, only the $850 \mathrm{ppm}$ treatment fit a linear trend (LR, $P=0.007$; Figure 4). Cup alone was significant, but with a lower effect size than date and its interactions (Table 2). 
Differences in DML were much smaller in the Aug 7 trial, but still showing a significant interaction between $\mathrm{pCO}_{2}$, date, and cup (Table 2;). As with the Jul 11 trial, all factors were significant here, but cup and date had the greatest effect sizes (Table 2). The effect of hatching date on DML was near significance in the $400 \mathrm{ppm}$ treatment and significant in both the 1900 and 2200 ppm treatments (Table S2). All of the $\mathrm{pCO}_{2}$ treatments demonstrated a non-linear (LR, 400: $P=0.8632 ; 1900: P=0.8733 ; 2200: P=0.5168)$ bimodal distribution of DML over hatching, with peaks on the first and fourth days of hatching (Figure 4).

The Aug 7 trial consisted of egg capsules from two separate adult holding tanks (tank A and tank B) sorted into the culture cups for each $\mathrm{pCO}_{2}$ treatment (Cup 1: AA, Cup 2: BB, Cup 3: $\mathrm{AB}$ ). Cup (nested in $\mathrm{pCO}_{2}$ treatment) had the greatest effect size on DML in this trial, while its interaction had the lowest (Table 2). At the scale of discrimination by cup, egg number could be notably relevant, so values are reported here while detailed statistical analyses can be found in the Supplementary Materials. In brief, egg number appears to be a significant covariate interacting with all other factors (cup [not nested when acting as a proxy for tank/egg capsule source], $\mathrm{pCO}_{2}$, and their interaction in particular, Table S7). Integrated across treatments, cup/source had a significant effect on egg number (ANOVA, $\mathrm{F}_{2,490}=284.7, \mathrm{P}<<0.001$ ) with all cups/sources being significantly different from each other (Tukey, $\mathrm{P}<0.05$; Cup 1/AA: $128.7 \pm$ 12.1 eggs/capsule; Cup 2/BB: $169.8 \pm 19.9$ eggs/capsule; Cup 3/AB: $147.0 \pm 14.9$ eggs/capsule)

Within the 400 ppm treatment, cup had a significant effect on paralarval DML (Table S3) with Cup 2/BB paralarvae significantly (Tukey, $P<0.05)$ larger $(1.64 \pm 0.12 \mathrm{~mm}, 192.5$ eggs/capsule) than those from Cup 1/AA (1.56 $\pm 0.12 \mathrm{~mm}, 117.5$ eggs/capsule) and Cup 3/AB ( $1.54 \pm 0.11 \mathrm{~mm}, 126$ eggs/capsule). The $1900 \mathrm{ppm}$ treatment also showed significant differences between cups (Table S3; Tukey, $P<0.05)$, but with Cup 1/AA paralarvae $(1.46 \pm$ $0.12 \mathrm{~mm}, 123 \mathrm{eggs} /$ capsule) much smaller than those from both Cup 2/BB (1.54 $\pm 0.11 \mathrm{~mm}, 144$ eggs/capsule) and Cup 3/AB (1.59 $\pm 0.13 \mathrm{~mm}, 158.5$ eggs/capsule). No difference was seen in the 2200 ppm treatment (Table S3; Cup 1/AA: $1.52 \pm 0.10 \mathrm{~mm}, 145.5$ eggs/capsule; Cup 2/BB: $1.56 \pm 0.12 \mathrm{~mm}, 173 \mathrm{eggs} /$ capsule; Cup 3/AB: $1.52 \pm 0.14 \mathrm{~mm}, 156.5$ eggs/capsule). Paralarvae from Cup 1/AA and Cup 2/BB showed similar patterns of response to the acidification exposure, a non-linear decrease with increased exposure (LR, Cup 1/AA: $P=0.4789$; Cup 2/BB: $P=$ 0.2190), while no trend (LR, $P=0.9514$ ) or clear pattern of response was seen in Cup 3/AB (Fig 
5). Compiled across cups, the data shows the relative decrease in DML, approaching significance, reported in data by trial (LR, $P=0.0824$; Fig 3, Fig 5).

Variance of the DML data, assessed by pooling each cup and comparing across treatments within a trial, consistently increased with increasing acidification. No individual ttests showed significant differences in variance between treatments, likely influenced by low sample size ( $\mathrm{n}=3$, two-sample $\mathrm{t}(2), P>0.05$ for all treatment pairings within each trial). All three trials demonstrated non-significant increasing linear trends in DML variance (Figure 3; LR, Jul 3: $P=0.1038$; Jul 11: $P=0.2297$; Aug 7: $P=0.1738$ ). The change in variance, relative to trial average, for all $\mathrm{pCO}_{2}$ treatments best fit $\left(\mathrm{R}^{2}=0.780\right)$ a two-line model breaking after 1300 ppm (no significant difference from stepwise model, $P=0.544$; Figure 3). In the Aug 7 trial, DML variance was highest in Cup 3/AB $\left(0.0159 \pm 0.0035 \mathrm{~mm}^{2}\right)$, but not significantly different from the other cups (two-sample t(2), $P>0.05$ for all pairings; Cup 1/AA: $0.0129 \pm 0.0020 \mathrm{~mm}^{2}$; Cup 2/BB: $0.0143 \pm 0.0011 \mathrm{~mm}^{2}$ ).

\section{Yolk sac volume}

Patterns of response in yolk sac volume were highly variable within and between trials (Figure 3). Yolk sac volume in the low/control treatments decreased markedly (one-way ANOVA, $F(2,471)=166.8, P<0.001)$ between the Jul 3 trial $\left(0.077 \mathrm{~mm}^{3}, 0.042-0.138 \mathrm{~mm}^{3}\right)$ and the Jul $11\left(0.030 \mathrm{~mm}^{3}, 0.017-0.054 \mathrm{~mm}^{3}\right)$ and Aug $7\left(0.029 \mathrm{~mm}^{3}, 0.020-0.044 \mathrm{~mm}^{3}\right)$ trials (Tukey, $\mathrm{P}<0.05$ ). Despite this, only cup, nested within $\mathrm{pCO}_{2}$ nested within trial, appears significant when data is compiled (Table 2). In the Jul 3 trial, $\mathrm{YV}$ decreased linearly across $\mathrm{pCO}_{2}$ treatments (LR, $P=0.017$; Figure 3 ) with the ESL Ambient/550 treatment having significantly larger YV (Table S1; Tukey, $P<0.05 ; 0.077 \mathrm{~mm}^{3}, 0.070-0.084 \mathrm{~mm}^{3}$ ) than the $2200 \mathrm{ppm}$ treatment $\left(0.058 \mathrm{~mm}^{3}, 0.034-0.100 \mathrm{~mm}^{3}\right)$. Conversely, yolk volume increased near-linearly (LR, $P=0.060$; Figure 3) with increasing acidification in the Jul 11 trial with the $850 \mathrm{ppm}$ treatment showing significantly smaller YV (Table S1; Tukey, $P<0.05 ; 0.030 \mathrm{~mm}^{3}, 0.017$ $\left.0.054 \mathrm{~mm}^{3}\right)$ than the $2200 \mathrm{ppm}\left(0.036 \mathrm{~mm}^{3}, 0.018-0.72 \mathrm{~mm}^{3}\right)$ treatment. Yolk sac volume was not affected by $\mathrm{pCO}_{2}$ in the Aug 7 trial (Table S1; Figure 3). In the compiled data, normalized to trial mean, piecewise regression showed a weakly fitting $\left(\mathrm{R}^{2}=0.221\right)$ two line model, not significantly different from a stepwise null model $(P=0.839)$ with a breakpoint between 850 and $1300 \mathrm{ppm}$ (Figure 3). Variance of the YV also showed no trends with increasing acidification 
(LR, $P>0.05$ for all trials; $\mathrm{n}=3$, two-sample $\mathrm{t}(2), \mathrm{P}>0.05$ for all treatment pairings within each trial) with piecewise regression revealing a two line best fit $\left(\mathrm{R}^{2}=0.609\right)$ model breaking at the lowest values at $1300 \mathrm{ppm}$ (no difference from stepwise null model, $P=0.304$; Figure 3).

The interaction of $\mathrm{pCO}_{2}$, date, and cup had a significant impact on $\mathrm{YV}$ in all three trials (Table 2). In the Jul 3 trial, the interaction of $\mathrm{pCO}_{2}$ with date had the greatest effect size (Table 2) showing trends in YV over hatching similar to the DML data, with the ESL Ambient $/ 550 \mathrm{ppm}$ (LR, $P=0.140$ ) increasing slightly, while the $1300 \mathrm{ppm}(\mathrm{LR}, P=0.038)$ and $2200 \mathrm{ppm}(\mathrm{LR}, P$ $=0.145$ ) paralarvae decrease (Figure 4). All factors and interactions were significant in the Jul 11 trial (Table 2), though weaker than the Jul 3 trial with cup appearing to be a stronger interacting factor with $\mathrm{pCO}_{2}$ than date. While $\mathrm{YV}$ significantly changed with date under the 850 and $2200 \mathrm{ppm}$ treatments (Table S2), no particularly strong trends were seen (LR, $P>0.05$; Figure 4). The Aug 7 trial showed no overall effect of $\mathrm{pCO}_{2}$, but a weak effect of date and near significant interaction of $\mathrm{pCO}_{2}$ and date (Table 2, Table S1). There were significant differences in YV with date in the 400 and $2200 \mathrm{ppm}$ treatments (Table S2), with all three treatments showing weakly increasing trends over hatching (LR, 400: $P=0.079$; 1900: $P=0.069 ; 2200: P$ $=0.144$; Figure 4$)$.

Cup and its interaction showed significant effects in the Aug 7 trial (Table 2). Since the correlation of egg number to YV is non-significant and very weak (Figure S4), statistical models are included in the Supplementary Materials, but are not reported here (Tables S7 \& S8). Cup 1/AA showed no significant difference with $\mathrm{pCO}_{2}$ (ANOVA, $\mathrm{F}_{2,153}=1.32, P=0.268$ ), but an increasing trend (LR, $P=0.031$; Figure 5). Cup 2/BB, conversely showed a significant decrease in $\mathrm{YV}$ at the $1900 \mathrm{ppm}$ level (ANOVA, $\mathrm{F}_{2,170}=8.36, P<0.001$; Tukey, $P<0.05$ ) driving a slight, but non-significant, decreasing trend (LR, $P>0.05$; Figure 5). Cup 3/AB showed no significant effect (ANOVA, $F_{2,148}=0.315, P=0.730$ ) and a very weakly increasing trend (LR, $P$ $>0.05$ ) in $\mathrm{YV}$ with increasing $\mathrm{pCO}_{2}$ (Figure 5). The $400 \mathrm{ppm}$ treatment varied by cup (Table S3; Tukey, $\mathrm{P}<0.05)$ with Cup 2/BB having greater YV $\left(0.034 \mathrm{~mm}^{3}, 0.023-0.050 \mathrm{~mm}^{3} ; 192.5\right.$ eggs/capsule) than both Cup 1/AA $\left(0.028 \mathrm{~mm}^{3}, 0.019-0.040 \mathrm{~mm}^{3} ; 117.5\right.$ eggs/capsule $)$ and Cup 3/AB $\left(0.026 \mathrm{~mm}^{3}, 0.017-0.038 \mathrm{~mm}^{3} ; 126\right.$ eggs/capsule $)$. Yolk volume variance did not differ between cups of the Aug 7 trial ( $\mathrm{n}=3$, two-sample $\mathrm{t}$ test, $t(2), P>0.05$ for all cup pairings). Based on a comparison of average values for each cup, yolk sac volume was independent of dorsal mantle length (LR, $P>0.05$, for all trials; Figure 6). 


\section{Hatching time}

Increasing acidification consistently delayed hatching in all trials (Figure 7). Days until hatching initiation, defined as the day at which $1 \%$ cumulative hatching occurred in at least one treatment, also increased across trials (Jul 3: 12 days from laying, Jul 11: 14 days, Aug 7: 15 days). In the Jul 3 trial, the proportions of cumulative hatching over time were significantly different between $\mathrm{pCO}_{2}$ treatments (G-test, ESL Ambient x 1300 ppm: $G(12)=156.2556, \mathrm{P}<<$ 0.0001; ESL Ambient x 2200 ppm: $G(12)=412.4811, \mathrm{P}<<0.0001 ; 1300 \times 2200$ ppm: $G(12)=$ 517.2413, $P<<0.0001)$. Cumulative hatching proportions also varied significantly between cups (G tests, $P<0.0001$ for all cup pairs within each $\mathrm{pCO}_{2}$ treatment, except 2200 ppm Cups 1 and 2, $P=0.2629$ ). Distributions of cumulative fraction hatched over time, compiled by $\mathrm{pCO}_{2}$ treatment, are considered here for concision (Figure 7). Hatching in the $2200 \mathrm{ppm}$ treatment was consistently delayed from the ESL Ambient and 1300 ppm treatments by about 1 day (Figure 7). Cumulative hatching reached at least $25 \%$ at 13 days from laying in the ESL Ambient and the 1300 ppm treatment, but took 14 days in the 2200 ppm treatment. Hatching of $75 \%$ or greater was reached 14 days from laying in the 1300 ppm treatment, 15 days in the ESL Ambient treatment, and 16 days in the 2200 ppm treatment.

Proportions of cumulative hatching over time, compiled by $\mathrm{pCO}_{2}$ treatment, were also significantly different within the Jul 11 trial $(850 \times 1300$ ppm: $G(11)=81.9224, P<<0.0001$; 850 x 2200 ppm: $G(12)=664.3269, \mathrm{P}<<0.0001 ; 1300$ ppm x 2200 ppm: $G(12)=500.7742, \mathrm{P}$ $<<0.0001)$. Again, some variability in hatching dynamics was seen between cups (G tests, $P<$ 0.01 for all cup pairs within each $\mathrm{pCO}_{2}$ treatment, except 2200 ppm Cups 1 and 2, $P=0.2880$ ). Compiled, the distributions show a consistent delay of about 1 day, expanding to 2 days, in the 2200 ppm treatment (Figure 7). Hatching reached at least 25\% 15 days after laying in the 850 and $1300 \mathrm{ppm}$ treatments and 16 days after in the $2200 \mathrm{ppm}$ treatment. Cumulative hatching of at least $75 \%$ was reached 16 days after laying in the 850 and 1300 ppm treatments and 18 days after laying in the $2200 \mathrm{ppm}$ treatment.

The Aug 7 trial also showed notable differences between $\mathrm{pCO}_{2}$ treatments in cumulative hatching proportions over time (400 x 1900 ppm: $G(15)=693.0624, P<<0.0001 ; 400 \times 2200$ ppm: $G(15)=892.6867, P<<0.0001 ; 1900$ ppm x 2200 ppm: $G(12)=79.242, P<<0.0001)$ and between the cups of each treatment (G tests, $P<0.05$ for all cup pairs within each $\mathrm{pCO}_{2}$ 
treatment, except 2200 ppm Cups 2 and 3,P=0.0564). A consistent delay of about 1 day was seen in the 1900 and $2200 \mathrm{ppm}$ treatments, compared to the $400 \mathrm{ppm}$ treatments (Figure 7). At least $25 \%$ cumulative hatching was seen in the 400 ppm treatment 16 days after laying, but 17 days after in the 1900 and $2200 \mathrm{ppm}$ treatments. At least $75 \%$ hatching occurred after 18 days in the $400 \mathrm{ppm}$ treatment, and after 19 days in the 1900 and $2200 \mathrm{ppm}$ treatments.

\section{Hatching success}

Hatching success was high across $\mathrm{pCO}_{2}$ treatments and trials, with at least $85 \%$ hatching always seen (compiled by treatment; Table S4). No trends in hatching success with increasing acidification were seen in any trial (Jul 3: LR, $P=0.8199$; Jul 11: LR, $P=0.2455$; LR, $P=$ 0.8431). Significant differences were seen in the distributions of staged, unhatched embryos and hatched paralarvae within treatments and cups in all trials (Table S4; G tests, $P<0.05$ ), but followed no pattern with acidification. In the Aug 7 trial, Cup 1 / AA had significantly higher embryonic mortality, particularly of middle and late stage embryos, than both Cup 2 / BB and Cup 3 / AB in both the 400 and 1900 ppm treatments ( $G$ tests, $P<0.05$ ); no differences were seen in the $2200 \mathrm{ppm}$ treatment ( $\mathrm{G}$ test, $P>0.05$ for all cup pairs). Occasional spikes in mortality of early stage embryos (e.g. 30.9\% of eggs in Cup 1 of the ESL Ambient / 550 treatment in the Jul 3 trial), either due to natural variability or faults of the culture system, may also have skewed results.

\section{Statolith morphometrics}

Statolith area broadly decreased with increasing acidification, although responses varied from trial to trial. In the Jul 3 trial, statoliths from the ESL Ambient $\left(6823.8 \mu \mathrm{m}^{2}, 6449.9\right.$ $7440.3 \mu \mathrm{m}^{2}$ ) treatment were significantly larger ( $\mathrm{KW}, H 2=9.0613, P=0.0108$; Dunn, $\mathrm{P}<0.05$ ) than those from the $1300 \mathrm{ppm}\left(5723.2 \mu \mathrm{m}^{2}, 5134.2-6620.3 \mu \mathrm{m}^{2}\right)$ and $2200 \mathrm{ppm}$ treatments (5803.2 $\mu \mathrm{m}^{2}, 5114.0-7142.8 \mu \mathrm{m}^{2}$ ), following an apparent step-wise drop (LR, $P=0.4090$; Figure 8). In the Jul 11 trial, statoliths from both the $850\left(7882.4 \mu \mathrm{m}^{2}, 7436.3-8115.9 \mu \mathrm{m}^{2}\right)$ and $1300 \mathrm{ppm}\left(7778.3 \mu \mathrm{m}^{2}, 7553.5-8017.7 \mu \mathrm{m}^{2}\right)$ treatments were much larger $(\mathrm{KW}, H 2=13.9475$, $P=0.0009$; Dunn, $\mathrm{P}<0.05)$ than those in the $2200 \mathrm{ppm}$ treatment $\left(4845.0 \mu \mathrm{m}^{2}, 3291.6-6747.8\right.$ $\mu \mathrm{m}^{2}$ ), again following a step-wise drop (Figure $8 ; \mathrm{LR}, P=0.3462$ ). There was no difference $(\mathrm{KW}, H 2=1.8239, P=0.4017)$ in statolith area between $\mathrm{pCO}_{2}$ treatments in the Aug 7 trial 
(400: $6814.4 \mu \mathrm{m}^{2}, 6218.0$ - $7074.4 \mu \mathrm{m}^{2} ; 1900: 6618.2 \mu \mathrm{m}^{2}, 5920.2$ - $7355.9 \mu \mathrm{m}^{2} ; 2200: 6473.3$ $\mu \mathrm{m}^{2}, 6119.3-6836.1 \mu \mathrm{m}^{2}$ ) and no trend in these data (Figure 8; LR, $P=0.7102$ ). Overall, relative to trial, statolith surface area best fit $\left(\mathrm{R}^{2}=0.638\right)$ a two-line model with a breakpoint at $1300 \mathrm{ppm}$ (at which area decreases), which did not significantly differ from a stepwise null hypothesis $(P=0.681$, Figure 8$)$. Statolith area appeared to be dependent on mantle length, based on a comparison of average values for each treatment, which approached significance (LR, $P=$ 0.0519, Figure S2).

The variance of the internal angle of the statolith outline, the metric of statolith edge rugosity, broadly increased with increasing acidification in the compiled data, driven by the Jul 11 samples. Internal angle variance was significantly higher $(\mathrm{KW}, H 2=17.6603, P=0.0001$; Dunn, $P<0.05)$ in the $1300 \mathrm{ppm}\left(507.11 \mathrm{deg}^{2}, 412.71-715.98 \mathrm{deg}^{2}\right)$ treatment of the Jul 3 trial than either the ESL Ambient (225.96 deg 2 , $\left.169.39-294.59 \mathrm{deg}^{2}\right)$ and the $2200 \mathrm{ppm}$ (277.83 $\mathrm{deg}^{2}, 130.13-577.08 \mathrm{deg}^{2}$ ) treatments resulting in a nonsignificant increasing trend (LR, $P=$ 0.8082; Figure 8). In the Jul 11 trial, treatments were not significantly different from each other (KW, $H 2=4.8128, P=0.0901$ ), but internal angle variance of the statoliths followed an increasing trend with acidification (850: $89.13 \mathrm{deg}^{2}, 63.38-367.81 \mathrm{deg}^{2} ; 1300: 151.25 \mathrm{deg}^{2}$, 88.30 - $308.43 \mathrm{deg}^{2}$; 2200: $348.32 \mathrm{deg}^{2}, 158.85$ - $521.12 \mathrm{deg}^{2}$; LR, $P=0.0252$; Figure 8). Statolith internal angle variance was much lower overall in the Aug 7 trial, and showed no differences between treatments $(\mathrm{KW}, H 2=4.0206, P=0.1339)$ and no particular trend with acidification (400: $97.51 \mathrm{deg}^{2}, 79.25-115.35 \mathrm{deg}^{2}$; 1900: $110.49 \mathrm{deg}^{2}, 92.63-129.89 \mathrm{deg}^{2}$; 2200: $97.44 \mathrm{deg}^{2}, 79.65-118.81 \mathrm{deg}^{2}$; LR, $P=0.5197$; Figure 8). The data compiled relative to trial means best fit a two-line model $\left(\mathrm{R}^{2}=0.716\right)$ with a breakpoint at $1300 \mathrm{ppm}$ (at which internal angle variance increases) that did not differ from a stepwise model $(P=0.277$; Figure 8).

The average variance of statolith surface pixel intensity $\left(\mathrm{px} \mathrm{int}{ }^{2}\right)$ followed similar patterns as internal angle variance, with a stepwise model $\left(\mathrm{R}^{2}=0.573, P=0.521\right)$ increasing at a 1300 ppm breakpoint in the compiled data; again driven by the Jul 11 samples. In the Jul 3 trial, average surface pixel variance was highest in the $1300 \mathrm{ppm}\left(1085.18 \mathrm{px} \mathrm{int}^{2}, 854.73-1386.61\right.$ px int ${ }^{2}$ ) treatment of the Jul 3 trial, significantly above (KW, $H 2=13.2045, P=0.0014$; Dunn, $P$ $<0.05)$ the ESL Ambient (665.77 px int $\left.{ }^{2}, 526.24-929.30 \mathrm{px} \mathrm{int}^{2}\right)$ and $2200 \mathrm{ppm}\left(713.83 \mathrm{px} \mathrm{int}^{2}\right.$, $448.52-849.16 \mathrm{px} \mathrm{int}^{2}$ ) treatments (LR, $P=0.8843$; Figure 8). The Jul 11 trial followed a stepwise jump in surface variation (LR, $P=0.2292$; Figure 8 ), with the statoliths of the 850 (185.41 
px int ${ }^{2}, 116.57-290.16$ px int $\left.^{2}\right)$ and 1300 ppm (200.95 px int ${ }^{2}, 155.71-282.51$ px int $\left.{ }^{2}\right)$ treatments having significantly lower surface variation $(\mathrm{KW}, H 2=16.0099, P=0.0003$; Dunn, $P$

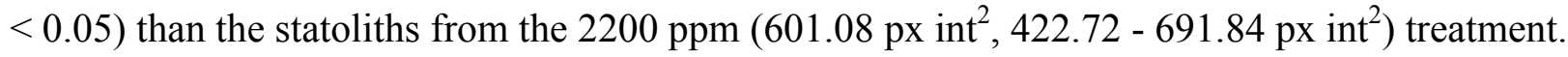
Statolith surface pixel variance was lower in the Aug 7 trial, although it still showed significant differences $(\mathrm{KW}, H 2=7.9688, P=0.0186$; Dunn, $P<0.05)$ between $\mathrm{pCO}_{2}$ treatments, with the 400 ppm treatment (111.24 px int $\left.{ }^{2}, 64.36-147.25 \mathrm{px} \mathrm{int}^{2}\right)$ having lower surface variation than the 1900 ppm (144.26 px int ${ }^{2}, 123.33$ - 169.51 px int $^{2}$ and 2200 ppm (130.42 px int ${ }^{2}, 100.26-$ $178.62 \mathrm{px} \mathrm{int}^{2}$ ) treatments, resulting in a weakly increasing trend with acidification (LR, $P=$ 0.1406; Figure 8).

Rectangularity and circularity of the statoliths were inversely related, demonstrating weak, non-significant trends with increasing acidification (LR, $P>0.05$; Figure 8). Compiled, rectangularity fit $\left(\mathrm{R}^{2}=0.426, P=0.791\right)$ a stepwise model decreasing at $1300 \mathrm{ppm}$. Circularity also best fit a stepwise model $\left(\mathrm{R}^{2}=0.657, P=0.319\right)$, but with a breakpoint increasing circularity between 850 and 1300 ppm. In the Jul 3 and Jul 11 trials, where statoliths showed impacts of acidification in other metrics, statoliths appear to become less rectangular and more circular (Figure 8). Statoliths from the $1300 \mathrm{ppm}$ treatment of the Jul 3 trial had significantly lower rectangularity than those from the ESL Ambient $/ 550$ treatment $(\mathrm{KW}, H 2=17.6603, P=$ 0.0001; Dunn, $P<0.05$; Figure 8), but this was the only result to support these potential trends, likely a factor of low sample sizes and high variability.

\section{Discussion}

This work expands our knowledge of the physiological impacts of ocean acidification on the early development of squid paralarvae, while also demonstrating the capacity for adaptation and resilience inherent to this fecund, plastic organism. In response to elevated $\mathrm{pCO}_{2}$, hatchling D. pealeii paralarvae demonstrated reduced mantle length, delayed hatching time, and degraded statoliths, consistent with the observations by Kaplan et al. (2013). Breakpoints in the compiled data were consistently around $1300 \mathrm{ppm} \mathrm{CO}_{2}$ across metrics, although there was notable variability in response strength from trial to trial. This value falls above IPCC predictions for ocean acidification in the open ocean by $2100(\sim 850 \mathrm{ppm})$, but below that for $2250(\sim 1500 \mathrm{ppm})$, and already occurs naturally, on short time scales, within estuarine and coastal systems (Caldeira and Wickett 2003; Doney et al. 2009; Baumann et al. 2015; Gledhill et al. 2015). Although 
juvenile and adult $D$. pealeii are known to enter estuarine systems, and thus tolerate some substantive $\mathrm{pH}$ variability, the eggs are typically laid in a more stable system: the nearshore shelf bottom up to $50 \mathrm{~m}$ depth (Gray 1992; Jacobson 2005). Even at the extremes of observed egg laying habitat, $\mathrm{pH}_{\mathrm{t}}$ should not be below 7.8 (about $700 \mathrm{ppm}$ in our system), but developing embryos appear capable of resisting acidification well beyond that mark (McMahon and Summers 1971; Jacobson 2005; Wang et al. 2013). It is likely, as has been observed for $D$. opalescens, that oxygenation delimits egg laying habitat as well as pH (Navarro et al. 2018). Oxygen should not be as restrictive on the Northwest Atlantic shelf, but perhaps for D. pealeii oxygen, or still other factors, is a more limiting determinant of the egg laying habitat window than $\mathrm{pH}$. Whereas thermal and hypoxia thresholds are often considered in physiological work, acidification thresholds have primarily been considered for calcifying marine organisms (Anthony et al. 2008; Byrne 2011; Gazeau et al. 2013; Rosa et al. 2013). However, a greater understanding of acidification tolerance windows in more marine organisms could be extremely useful for informing models and producing more robust predictions for fisheries management (Hofmann and Todgham 2010).

Depending on the mechanism through which $\mathrm{pH}$ balance is achieved, an organism may reach its limit through either increasing energetic costs or through the accumulation of bicarbonate (Fabry et al. 2008). Cephalopods are highly effective at $\mathrm{pH}$ balancing through ion transport, but this process is considered energetically costly (Hu et al. 2011b, 2013). The results presented here indicate an OA threshold for the case of embryonic $D$. pealeii, which have a finite energy reserve, but this "threshold" may not apply to post-hatch paralarvae and later stages of development which are potentially capable of moving out of stressful $\mathrm{pH}$ environments and may supplement energy through feeding (Vidal and Haimovici 1998; Bartol et al. 2008). Similarly, although hatching success was consistently high across trials and treatments, this only acts as a measure of embryonic survival and we cannot make any claims regarding the viability or survival of the resultant hatchlings.

\section{Energy budgets under stress: mantle length and yolk reserves}

The squid in each trial of this experiment demonstrated a different strategy of energy budget management in response to OA stress. In all cases, development rate was slowed, consistent with the observations of other loliginid embryos under acidification (Kaplan et al. 
2013; Rosa et al. 2014a; Navarro et al. 2016). It is uncertain if this developmental delay is a result of metabolic depression, which is a common response of marine invertebrates (Pörtner et al. 1998; Michaelidis et al. 2005). While metabolic depression under increased $\mathrm{pCO}_{2}$ (around 1000 ppm) has been observed in adult Humboldt squid, Dosidicus gigas, more recent research indicated that neither adults and juveniles of these squid nor of $D$. pealeii demonstrate metabolic depression or oxygen limitation under hypercapnia (1410 ppm; Rosa and Seibel 2008; Birk et al. 2018). Energy may have been sacrificed from growth in our experiments, as dorsal mantle length decreased with increasing OA in all trials. Yolk volume, however, responded in numerous ways, perhaps a result of varying resiliency, varying coping strategies, or yolk usage being inconsistently affected by $\mathrm{pCO}_{2}$ level (Figure 6).

Comparisons of mantle length and yolk volume highlight potential differences in the response to OA stress across the breeding season. In the Jul 3 trial, both DML and YV decrease slightly with increasing acidification suggesting a stressed system that requires more energy to maintain (Figure 6). In the Jul 11 trial, DML decreases, but YV slightly increases, as acidification increases suggesting a system of depressed metabolism/growth (Figure 6). Responses were low in the Aug 7 trial, with YV staying constant as DML slightly decreased with increasing acidification, suggesting either a potentially resilient system or a reduced impact magnitude due to the overall smaller paralarvae in this clutch (Figure 6).

While DML effect size was small, in context of the typical D. pealeii paralarvae it accounted for an approximately $5 \%$ reduction in size across trials as a result of acidification (integrated over hatching days). Raising $D$. pealeii paralarvae in captivity is a possible, but systemically challenging proposition, so while we unfortunately do not have direct observations of survival in this study we can hypothesize about the multiple pathways through which a reduction of this magnitude could impact the viability and survival of the hatchlings (Vidal et al. 2002b; Steer et al. 2003). The post-hatch transition from consumption of yolk reserves to prey capture is considered a critical period for squid paralarvae, and hatchling size is considered an important factor in prey capture success (Vidal et al. 2002a, b). Further, paralarval hydrodynamics and swimming speeds could be impacted by shifts in overall size, potentially impairing an already low (40\%) ability to escape predation (Bartol et al. 2008; York and Bartol 2016). Yolk volume reduction was seen only in the Jul 3 trial, but showed an average $24 \%$ decrease, compounding concerns for paralarval survival of the critical period under that response 
to acidification stress. Yolk content is also connected to paralarval specific gravity, and has been noted as of potential importance in paralarval survival as part of dispersal (Martins et al. 2010a).

Dorsal mantle length and yolk volume were often strongly affected by hatching date, indicating either natural variability in hatching dynamics and/or an impact of increased exposure time. The latter could be compounded by the delay in hatching time caused by increased acidification. Assuming growth rate for all embryos is consistent and occurs under the same conditions, mantle length would be expected to increase with hatching date, as the embryos that were not triggered to hatch continue to grow (Figure 4: ESL Ambient / 550). This model of development has been shown in the eggs of bigfin reef squid, Sepioteuthis lessioniana (Ikeda et al. 1999). Conversely, seeing a decrease in mantle length as hatching continues indicates embryos that either felt a greater impact of the stressor, lagged in development, and/or lacked in resources (Figure 4: 2200 ppm; Figure 4).

We expect that yolk would be consumed as hatching day increased, perhaps to a greater extent for paralarvae under stress. The Aug 7 trial however, broadly showed increases in yolk volume with hatching date in all treatments. Yolk utilization in squid paralarvae is known to be impacted by temperature, driving metabolism, and feeding state (Vidal et al. 2002a; Martins et al. 2010b). Both these factors were consistent across trials and so do not account for the different patterns in yolk utilization seen. Further, assessments of either varying development or yolk utilization rate are confounded by potential differences in maternal ration. Unfortunately, it is not

feasible to quantify yolk rations within a capsule without disturbing the embryos and potentially inducing premature hatching. Because maternity was unknown, and potentially mixed, within the egg mops used in each trial, it is possible that differences in maternal investment account for these variable patterns of response across the breeding season (Steer et al. 2004).

\section{Construction of the statolith}

Responses of the statolith to acidification followed similar patterns from trial to trial and were fairly consistent across the metrics observed. Statolith length has been correlated to mantle length in squid, so the decrease in statolith area seen with increasing acidification in our data is likely driven by the concurrent decrease in dorsal mantle length (Figure S1) (Ikeda et al. 1999; Steer et al. 2003). However, decreases in statolith area due to combined acidification and 
hypoxia described in D. opalescens were independent of paralarval size, so in certain stressor scenarios, statolith and organism size may be decoupled (Navarro et al. 2016).

The increases in statolith edge rugosity and surface porosity/malformation with increasing acidification (seen primarily in the Jul 11 trial) described by the metrics introduced here reflect the results described in Kaplan et al. (2013). Squid statoliths are constructed through the growth of long, thin aragonite crystals from a core nucleation site within a protein matrix that directs the construction and expansion of the statolith (Radtke 1983). The aragonite crystals were long and thin, indicating a good calcification environment (high $\mathrm{pH}$ and aragonite saturation state) within the statocyst, suggesting that the disorientation of crystals and surficial degradation seen was instead an effect of decreased expression or activity of matrix proteins (Cohen and Holcomb 2009). Tests of paralarval swimming behavior, run in parallel to these experiments, demonstrated impacts of acidification on the energetics of swimming (primarily speed and vertical stationing), but did not show impairment to the paralarvae's ability to orient themselves or any aberrant swimming behaviors under hypercapnia (Zakroff et al. 2018). Given reported, dramatic responses of cephalopod paralarvae swimming behavior when statoliths are severely malformed or absent and hair cells are malfunctioning, these data suggest that despite observed statolith degradation, statocyst function may not have been severely impaired (Colmers et al. 1984; Hanlon et al. 1989; Zakroff et al. 2018). Due to the limitations of the image-based analyses performed, only a surficial description of the hatchling statolith can be considered. In further studies, it would be worthwhile to examine deeper layers or the density of the statolith to see when during embryonic development construction is disrupted by external stress.

\section{A broader squid context}

Many of the previous studies of OA and squid showed repeated significant effects on an array of variables (Lacoue-Labarthe et al. 2011; Kaplan et al. 2013; Hu et al. 2014; Rosa et al. 2014a; Navarro et al. 2016). Here, we had trials that were affected by relatively high levels of $\mathrm{pCO}_{2}$ and low $\mathrm{pH}_{\mathrm{t}}$, but also trials that were not. This suggests some resiliency or tolerance of these squid to OA, at least during embryonic development. Indeed, these animals are tolerant of the naturally high $\mathrm{pH}$ and low oxygen concentrations of the egg capsule (Long et al. 2016). These results align with the limited, variable impacts of OA seen in D. opalescens embryos and are not unexpected when considering the relative $\mathrm{pCO}_{2}$ tolerance seen in juveniles and adults of 
D. pealeii and D. gigas (Rosa and Seibel 2008; Seibel 2015, 2016; Navarro et al. 2016; Birk et al. 2018). Upregulation of key proton secretion pathways in response to dramatic acidification (pH 7.31) in Sepioteuthis lessioniana embryos also reinforces the scope for $\mathrm{pH}$ regulation and OA tolerance in this group (Hu et al. 2013). In squids, physiological resiliency to OA may be species-specific, influenced by parental environments, and/or under the influence of other unknown factors. Importantly, behavioral sensitivity to OA has been shown in adult Idiosepius pygmaeus, which, while not a teuthid squid, highlights the potential for neurologically driven impacts on these organisms that were not examined here (Spady et al. 2014).

It has been suggested that marine invertebrates that produce egg capsules containing high numbers of embryos have a substantial capacity for plasticity (Oyarzun and Strathmann 2011). Cephalopods are broadly considered plastic organisms, altering their life history and population structure under different environmental factors (Pecl et al. 2004a; Pecl and Jackson 2008; Rosa et al. 2014b). Reproductive strategy and investment are also suggested to be highly plastic in cephalopods, and are likely influenced by parental environment (Pecl and Moltschaniwskyj 2006; Guerra et al. 2010; Robin et al. 2014). The dynamic variability in patterns of response to acidification across metrics and trials demonstrated here might be a product of this squid's high fecundity and patent plasticity.

As indicated by the potential relationship between our metrics and egg number, the variability between culturing cups may act as an extension of variability between egg capsules. Variability in the offspring of a single maternal clutch has been noted in the statoliths and DML of S. lessioniana (Ikeda et al. 1999). Notable egg capsule variability has also been described in D. opalescens, particularly in terms of statolith elemental composition (Navarro et al. 2014, 2016). In the Aug 7 trial, variability between cups represented a very basic means of differentiating parentage, maternity in particular, with embryos from tank B having slightly larger paralarvae with slightly greater yolk (from a greater number of eggs per capsule) than tank A. Squid are not known to maintain reserves of energy, not even for reproduction. Investment in reproduction primarily depends on the tradeoff between overall somatic growth and the development of the reproductive organs (Pecl and Moltschaniwskyj 2006). Production of eggs is fueled by energy captured through feeding and so fecundity is linked with adult mantle length, as size acts as an indicator of both energy intake potential and prey capture success (Boyle et al. 1995; Collins et al. 1995). While degradation of maternal investment in successive clutches has 
been demonstrated in some cephalopods, how a female squid distributes available energy among eggs and between egg capsules of a single clutch is not well described to our knowledge, particularly among the multiple egg per capsule squids (Steer et al. 2004).

Variation in offspring sensitivity to OA due to parental conditioning and epigenetics has been described in fishes, often relating to seasonal variation in the population (Miller et al. 2012; Murray et al. 2014; Schunter et al. 2016, 2018). Seasonal effects on sensitivity to OA have also been described in L. vulgaris, with winter stock proving more resistant to both acidification and warming (Rosa et al. 2014a). The distinctly different response patterns seen, across all metrics, between trials suggests that some form of higher scale variability is occurring within the $D$. pealeii sensitivity to OA stress. Doryteuthis pealeii has a roughly described, more anecdotally/locally acknowledged, succession of size classes, which may be cohorts, across its breeding season (Arnold et al. 1974; Mesnil 1977). Since these population dynamics are not well discriminated, a single year's sampling is not substantive enough to determine whether the variation seen between trials represents a consistent effect of seasonality/cohort on sensitivity to acidification stress. Further work would require more replications over the course of the breeding season to parse out this variability.

In an organism as dynamic and complex as D. pealeii there are multiple scales of variability to consider in assessing a physiological response to a stressor. This experiment served to highlight small-scale variabilities: those between individuals, cups, days, and trials. These results also highlight the importance of repetition and replication in organismal climate change response studies, particularly with organisms that have a high potential for plasticity. As evidenced here, neither data from a single trial nor data compiled across trials completely represented the scope of this animal's sensitivity and tolerance to acidification (Figure 3, Figure 8). Further, dynamics of life history must be considered in sampling, as parsing the data across days of hatching demonstrated. At several points, across trials, had only certain days been sampled or only integrated data across days been reported, the full dynamics of the stress response would not have been revealed (Figure 4). Investigation into sources of variability such as culture cup (which may relate to a previously undescribed relationship with egg number) served to emphasize aspects of reproductive and population biology that are still not well understood in this taxon and help guide needed future work. Examination of data at all of these scales is valuable, although each may have its own utility, but it is particularly worthwhile to 
examine these complex, frankly messy, systems as a whole as we attempt to understand and predict how these organisms will fare in a rapidly changing ocean.

\section{List of Abbreviations}

2D - Two-dimensional

DML - Dorsal mantle length

ESL - Environmental Systems Laboratory

KW - Kruskal-Wallis test

LR - Linear Regression

MBL - Marine Biological Laboratory

OA - Ocean acidification

SEM - Scanning electron microscopy

YV - Yolk Volume

\section{Funding}

This material was based upon work supported by the National Science Foundation Graduate Research Fellowship under Grant No. 1122374 to CZ. This project was funded by National Science Foundation Grant No. 1220034 to TAM.

\section{Acknowledgments}

We'd like to thank D. Remsen, the MBL Marine Resources Center staff, and MBL Gemma crew for their help acquiring squid. R. Galat and WHOI facilities staff provided system support. D. McCorkle, KYK Chan, and M. White provided guidance and insight on the acidification system and water quality monitoring. A. Solow provided statistics advice. We thank L. Kerr and the MBL Central Microscopy Facility for their aid with the SEM. We greatly appreciate E. Bonk, S. Zacarias, M. Lee, and A. Schlunk for their outstanding advice and assistance with this experiment.

\section{Bioethics}

All applicable international, national, and/or institutional guidelines for the care and use of cephalopods were adhered to. 


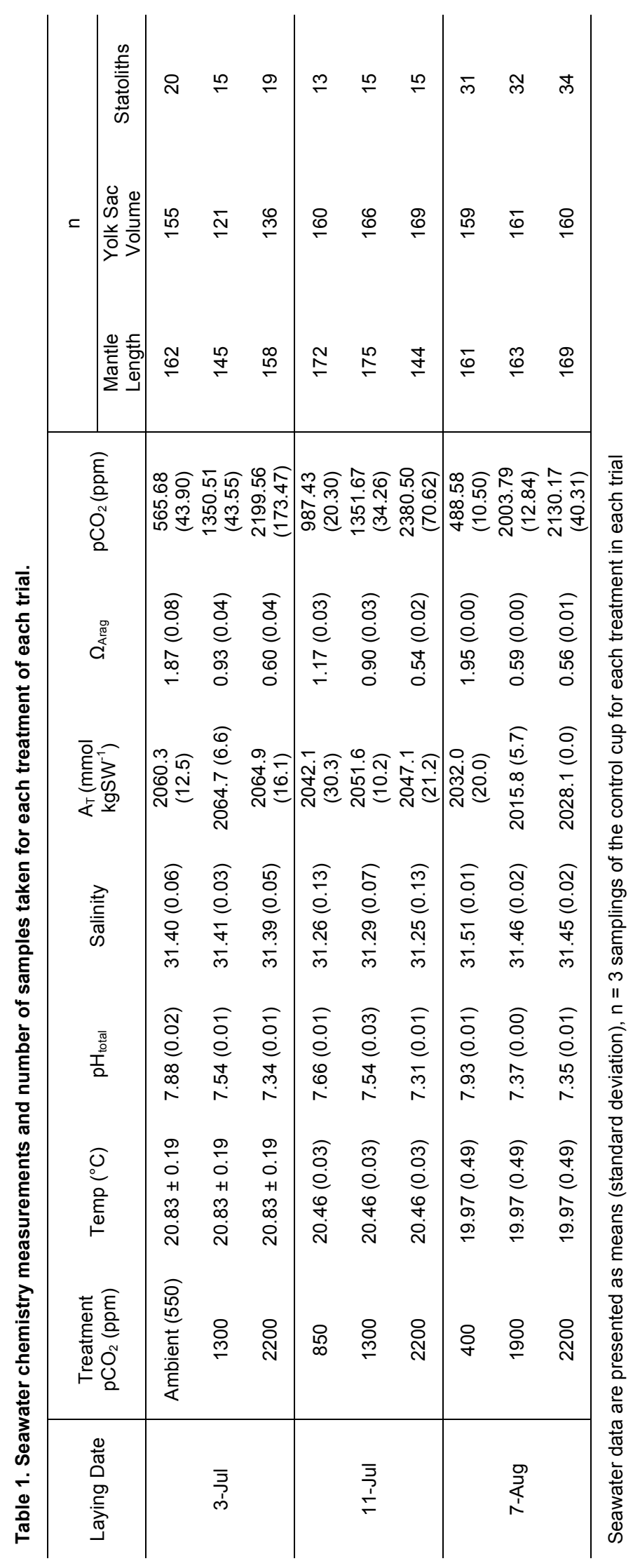


Table 2. Three-way Type II nested ANOVAs for compiled data and individual trials, for both mantle length and (logtransformed) yolk volume. Significant $p$ values $(\alpha=0.05)$ in bold.

\begin{tabular}{|c|c|c|c|c|c|c|c|c|c|c|}
\hline \multirow[b]{2}{*}{ Source } & \multicolumn{5}{|c|}{ Mantle Length } & \multicolumn{5}{|c|}{ Yolk Sac Volume } \\
\hline & SS & df & $F$ & $P$ & $\Omega^{2}$ & SS & df & $F$ & $P$ & $\Omega^{2}$ \\
\hline \multicolumn{11}{|l|}{$\begin{array}{l}\text { Compiled } \\
\text { Data }\end{array}$} \\
\hline Trial & 0.418 & 2 & 17.39 & $<0.001$ & 0.013 & 0.002 & 2 & 1.478 & 0.229 & 0.000 \\
\hline Trial : $\mathrm{pCO}_{2}$ & 0.562 & 15 & 3.121 & 0.075 & 0.013 & 0.003 & 15 & 0.243 & 0.983 & $\overline{0} 005$ \\
\hline Trial : Date & $-2.27^{*} 10^{-9}$ & 51 & $-3.703^{*} 10^{-9}$ & 1.000 & -0.020 & $1.4^{*} 10^{-5}$ & 51 & $3.44^{*} 10^{-4}$ & 1.000 & $\overline{-} 0.023$ \\
\hline $\begin{array}{l}\text { Trial : } \mathrm{pCO}_{2} \text { : } \\
\text { Date }\end{array}$ & 9.599 & 255 & 3.135 & 0.077 & 0.214 & 0.429 & 255 & 2.164 & 0.142 & 0.136 \\
\hline $\begin{array}{l}\text { Trial : } \mathrm{pCO}_{2} \text { : } \\
\text { Cup }\end{array}$ & 3.355 & 36 & 7.760 & $<0.001$ & 0.096 & 0.238 & 36 & 8.501 & $<0.001$ & 0.128 \\
\hline Residual & 16.55 & 1378 & & & & 1.023 & 1316 & & & \\
\hline \multicolumn{11}{|l|}{ Jul 3} \\
\hline $\mathrm{pCO}_{2}$ & 0.001 & 2 & 0.048 & 0.828 & -0.003 & $-4.836^{*} 10^{-12}$ & 2 & $-1.615^{*} 10^{-9}$ & 1.000 & 0.000 \\
\hline Date & 0.066 & 5 & 1.296 & 0.271 & 0.002 & 0.039 & 5 & 5.253 & 0.001 & 0.042 \\
\hline $\mathrm{pCO}_{2}$ : Date & 0.563 & 10 & 5.491 & $<0.001$ & 0.070 & 0.169 & 10 & 11.13 & $<0.001$ & 0.180 \\
\hline $\mathrm{pCO}_{2}:$ Cup & 0.576 & 6 & 9.363 & $<0.001$ & 0.078 & 0.078 & 6 & 8.730 & $<0.001$ & 0.084 \\
\hline $\begin{array}{l}\mathrm{pCO}_{2}: \text { Date } \\
\text { : Cup }\end{array}$ & 1.150 & 30 & 3.739 & $<0.001$ & 0.127 & 0.105 & 30 & 2.330 & $<0.001$ & 0.111 \\
\hline Residual & 4.256 & 415 & & & & 0.548 & 366 & & & \\
\hline \multicolumn{11}{|l|}{ Jul 11} \\
\hline $\mathrm{pCO}_{2}$ & 0.162 & 2 & 7.473 & $<0.001$ & 0.018 & 0.007 & 2 & 5.222 & 0.006 & 0.015 \\
\hline Date & 0.908 & 5 & 16.80 & $<0.001$ & 0.111 & 0.010 & 5 & 3.108 & 0.009 & 0.018 \\
\hline $\mathrm{pCO}_{2}$ : Date & 0.895 & 10 & 8.274 & $<0.001$ & 0.103 & 0.013 & 10 & 1.990 & 0.033 & 0.017 \\
\hline $\mathrm{pCO}_{2}:$ Cup & 0.233 & 6 & 3.589 & 0.002 & 0.022 & 0.024 & 6 & 6.189 & $<0.001$ & 0.054 \\
\hline $\begin{array}{l}\mathrm{pCO}_{2}: \text { Date } \\
\text { : Cup }\end{array}$ & 0.669 & 30 & 2.061 & 0.001 & 0.045 & 0.038 & 30 & 1.860 & 0.004 & 0.044 \\
\hline Residual & 4.759 & 440 & & & & 0.290 & 441 & & & \\
\hline \multicolumn{11}{|l|}{ Aug 7} \\
\hline $\mathrm{pCO}_{2}$ & 0.326 & 2 & 13.81 & $<0.001$ & 0.036 & $4.81 * 10^{-4}$ & 2 & 1.670 & 0.190 & 0.002 \\
\hline Date & 0.828 & 5 & 14.06 & $<0.001$ & 0.091 & 0.005 & 5 & 7.608 & $<0.001$ & 0.057 \\
\hline $\mathrm{pCO}_{2}$ : Date & 0.457 & 10 & 3.874 & $<0.001$ & 0.040 & 0.002 & 10 & 1.665 & 0.095 & 0.012 \\
\hline $\mathrm{pCO}_{2}:$ Cup & 1.054 & 6 & 14.90 & $<0.001$ & 0.116 & 0.005 & 6 & 5.590 & $<0.001$ & 0.048 \\
\hline $\begin{array}{l}\mathrm{pCO}_{2}: \text { Date } \\
\text { : Cup }\end{array}$ & 0.603 & 30 & 1.705 & 0.013 & 0.029 & 0.008 & 30 & 1.806 & 0.007 & 0.042 \\
\hline Residual & 5.186 & 440 & & & & 0.062 & 428 & & & \\
\hline
\end{tabular}



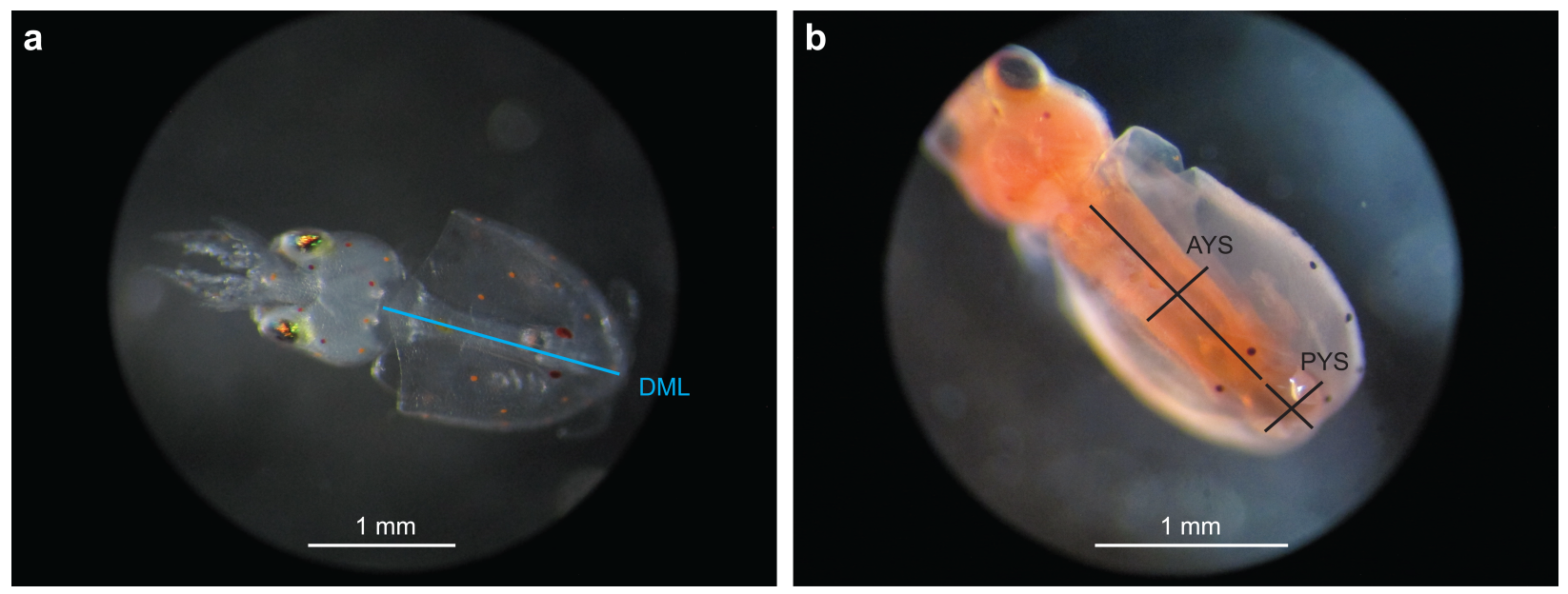

Figure 1. Doryteuthis pealeii paralarvae imaged for measurements of dorsal mantle length and yolk sac volume. a An anaesthetized paralarva photographed for measurement of its dorsal mantle length (DML, superimposed cyan line). b A preserved paralarva stained with oil red O photographed for measurement of its yolk sac volume. Length and width (superimposed black lines) of the anterior yolk sac (AYS) and posterior yolk (PYS) were measured to calculate total yolk volume. Scale bars are unique to each image, both representing $1 \mathrm{~mm}$. Photos by $\mathrm{CZ}$ 


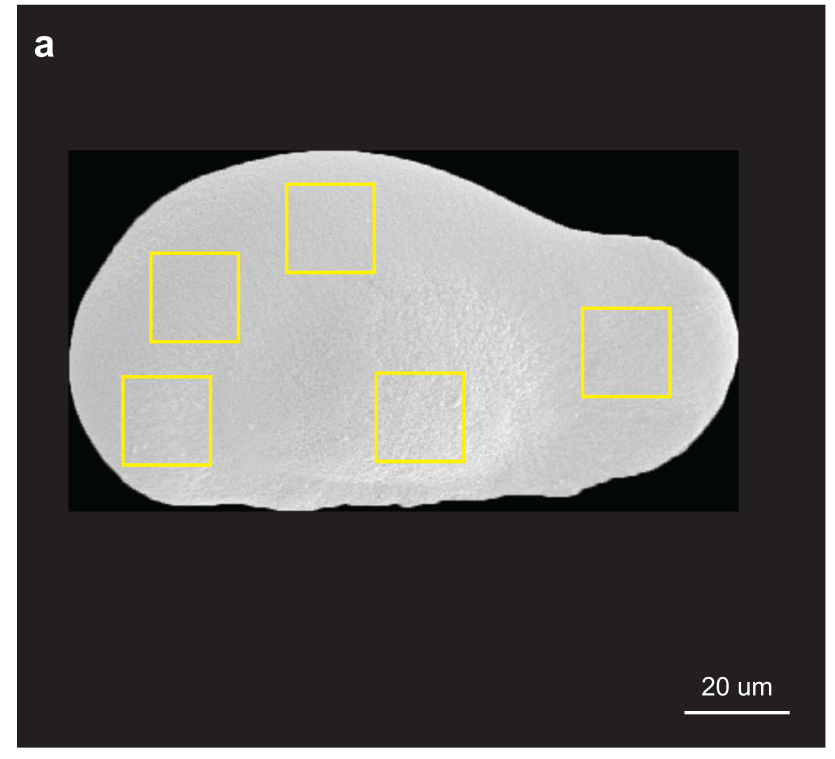

b

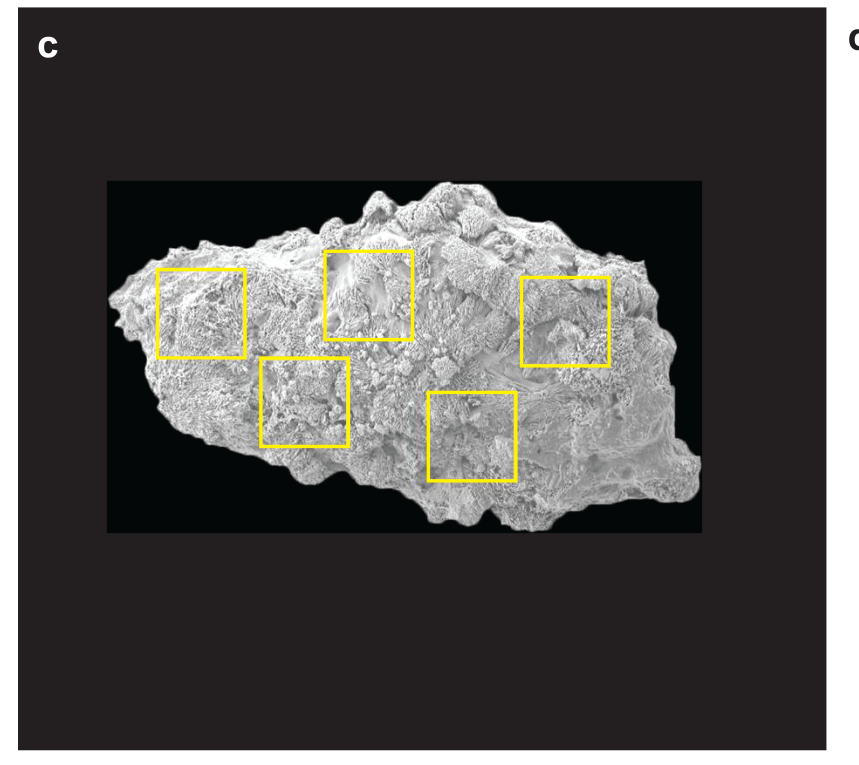

d

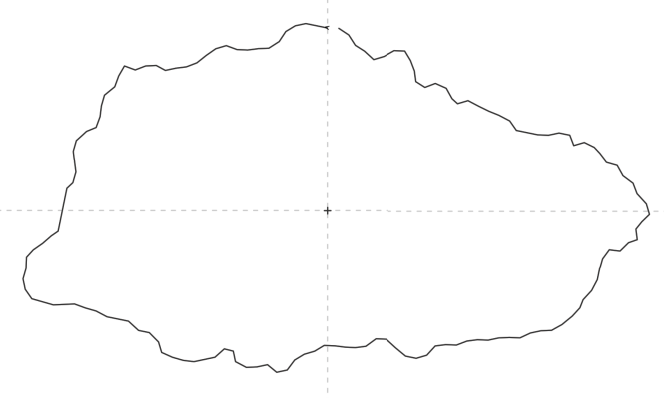

Figure 2. Statoliths extracted from control and high acidification exposures measured for size, shape, and surface variability. a A statolith from the $400 \mathrm{ppm} \mathrm{CO}_{2}$ treatment with the MATLAB surface analysis squares superimposed in yellow and $\mathbf{b}$ its outline produced in the R Momocs package. c A statolith from the 2200 ppm treatment with analysis squares superimposed and d its outline from Momocs. All images are to the same $20 \mu \mathrm{m}$ scale, shown in a 


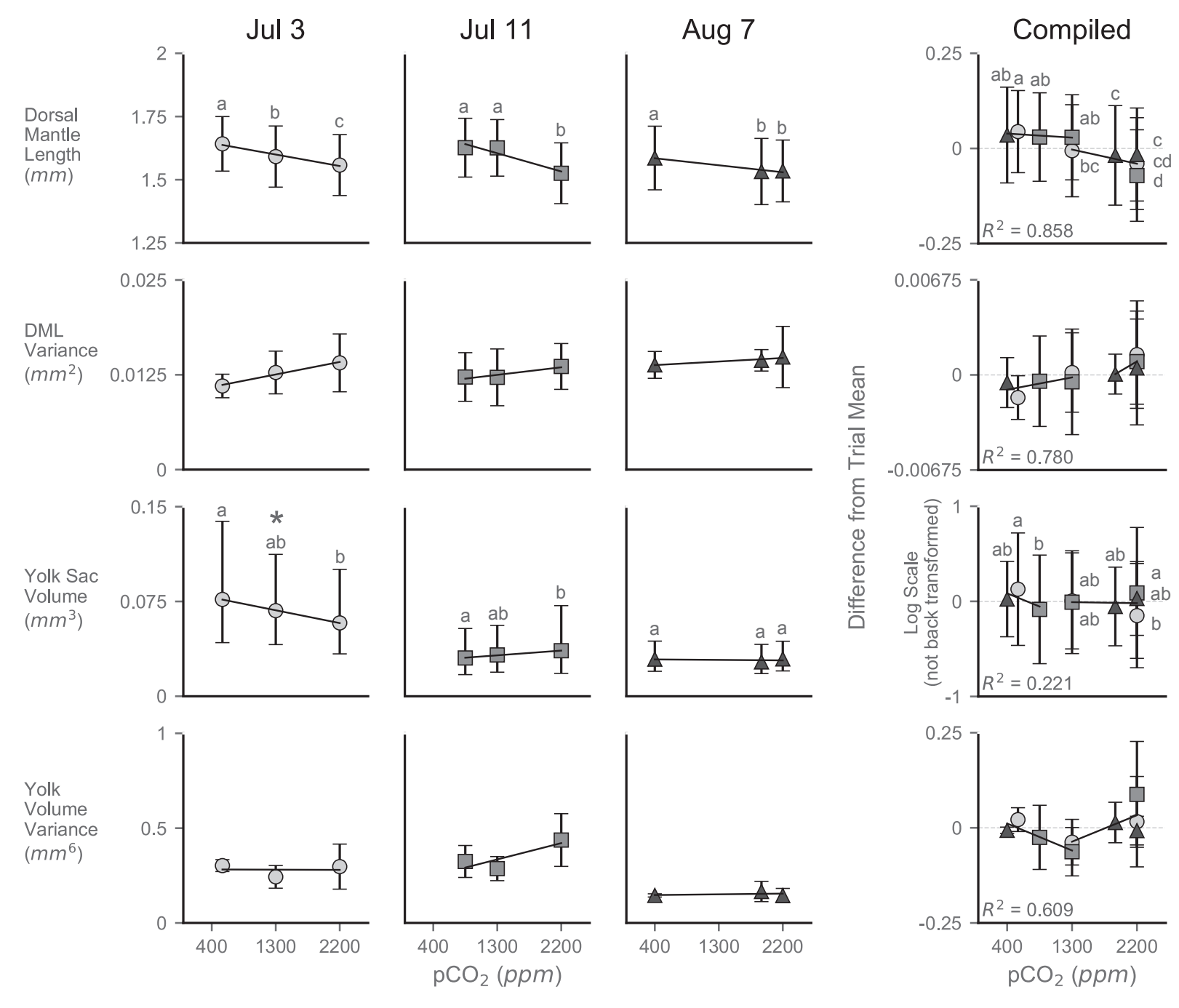

Figure 3. Dorsal mantle length, yolk sac volume, and respective variances of paralarvae exposed to a range of $\mathrm{pCO}_{2}$ treatments. Data are presented separated by trial (demarcated by egg capsule laying date) compiled across cups and hatching days for each $\mathrm{pCO}_{2}$ treatment (metric n's in Table 1, variance $n=3$ cups per treatment per trial). The Compiled plot depicts the data from all trials normalized by taking sample values and subtracting its respective trial mean. Differences in log transformed yolk sac volume data are not back transformed. Symbols represent means, with shape and color corresponding to trial. Error bars represent one standard deviation. Letters demarcate statistical groupings from a Tukey's HSD. Trend lines in trial data depict linear regressions; significance is marked with an asterisk $(P<0.05)$. Models of best fit from piecewise regressions are presented on compiled data with corresponding $\mathrm{R}^{2}$ 
Figure 4. Mean dorsal mantle length and yolk sac volume (back transform of logarithmic mean) of paralarvae across sampled hatching days. Measurements for the Jul 3, Jul 11, and Aug 7 trials are compiled across cups and presented by $\mathrm{CO}_{2}$ treatment; $\mathrm{n} \sim 30(\sim 10$ per experimental cup) paralarvae per symbol. Symbols represent means, with shape and color corresponding to $\mathrm{pCO}_{2}$ treatment (ppm). Error is not shown for visual clarity. Linear regressions are colored corresponding to their $\mathrm{pCO}_{2}$ treatment; significance is marked with an asterisk next to $\mathrm{pCO}_{2}$ treatment in the legend 

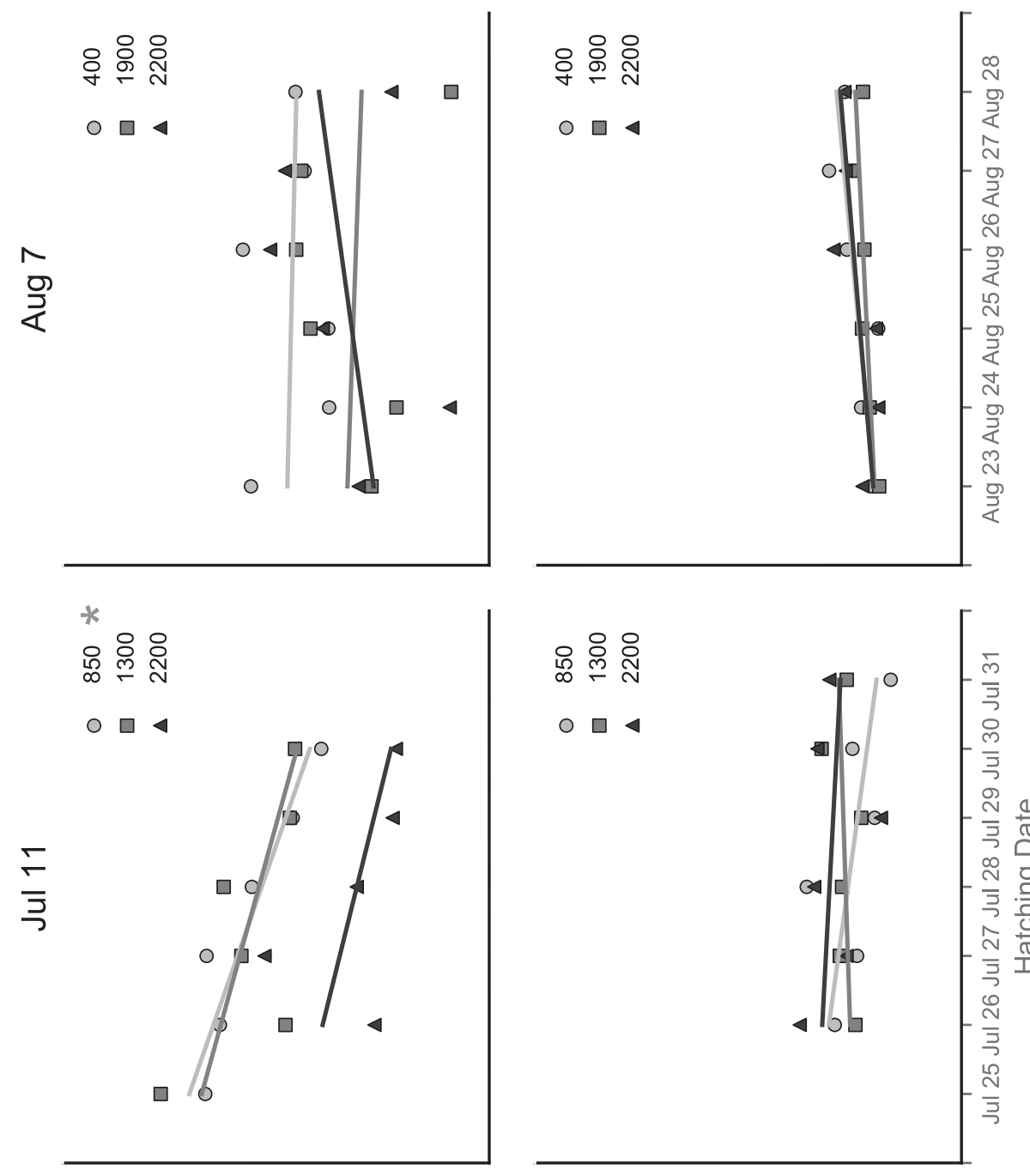

양 윰 오ํ

○ 4
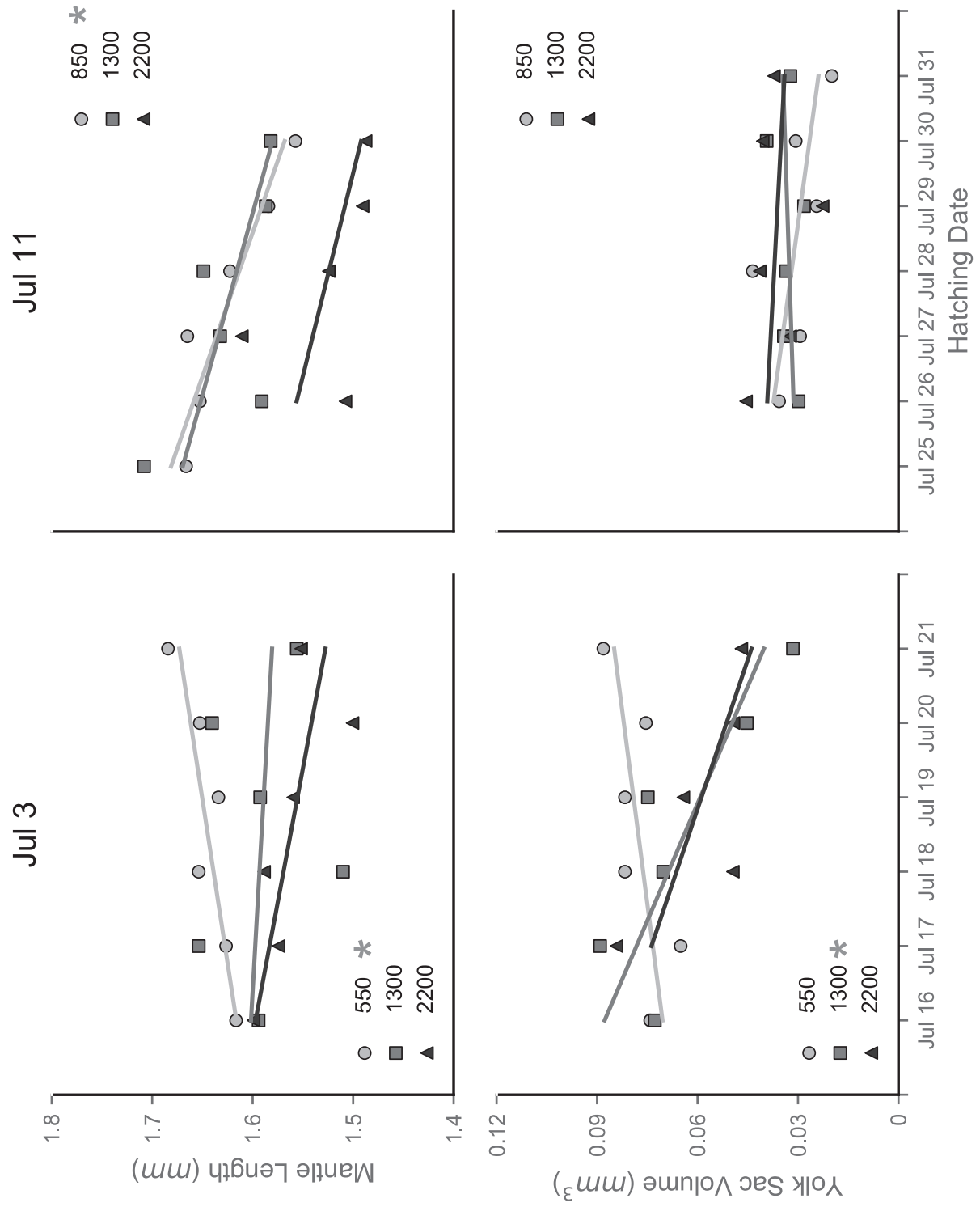


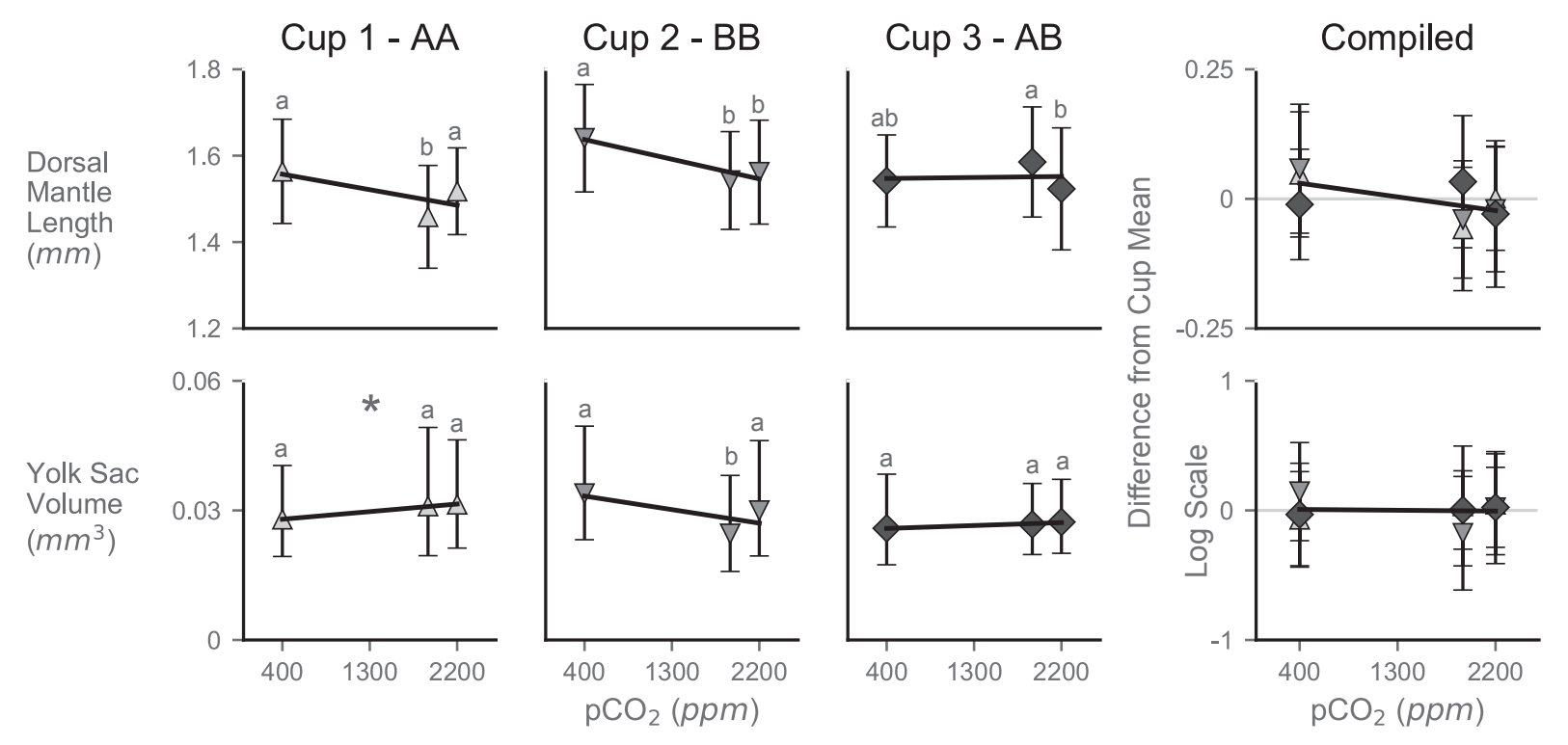

Figure 5. Dorsal mantle length and yolk sac volume (back transformed from logarithmic data) of Aug 7 trial paralarvae separated by culture cup. Cups in the Aug 7 trial each contained two egg capsules sorted from two separate adult squid tanks, tank A and tank B (Cup 1 = AA, Cup $2=$ $\mathrm{BB}$, and Cup $3=\mathrm{AB}$ ). The Compiled plot depicts the data from all cups normalized by taking sample values and subtracting its respective cup mean. Differences in log transformed yolk data are not back transformed. Symbols represent means, with shape and color corresponding to cup. Error bars represent one standard deviation; $n=\sim 53$ paralarvae per symbol $(\sim 10$ per day for 6 days, often fewer in the latter days of hatching). Letters demarcate statistical groupings from a Tukey's HSD. Lines depict linear regressions; significance is marked with an asterisk $(P<0.05)$ 


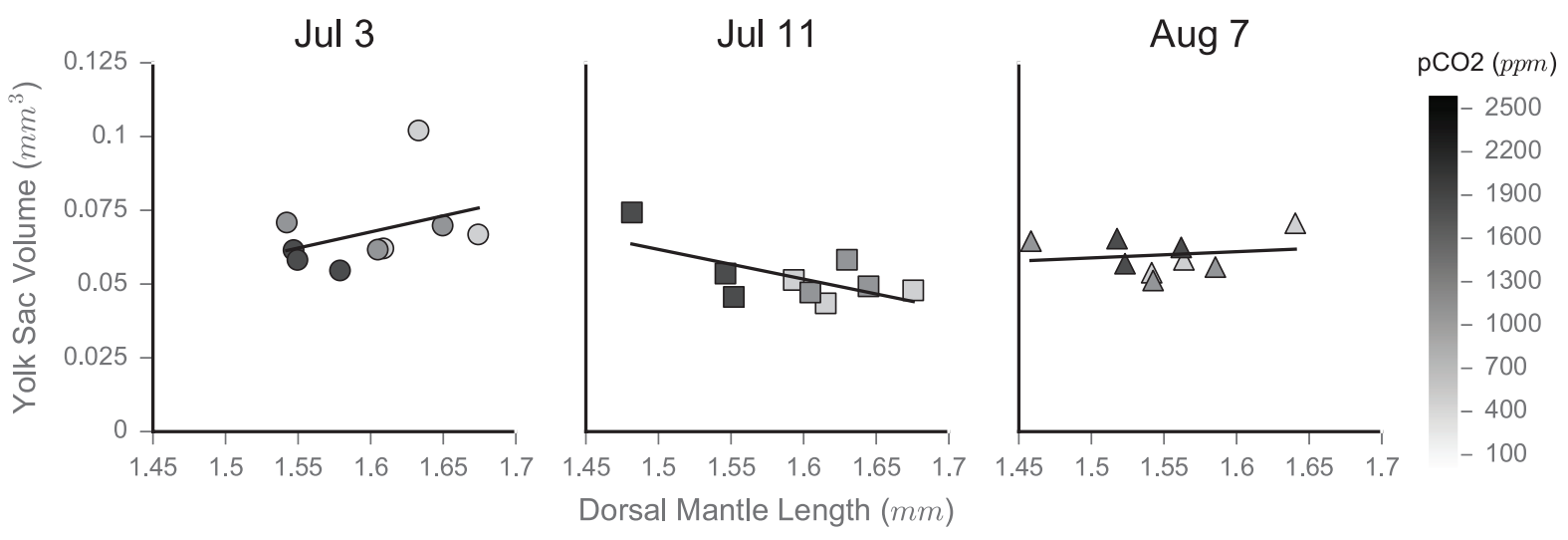

Figure 6. Comparison of average yolk sac volume and average mantle length. Data are averaged for each culture cup and are presented separated by trial; $n=3$ experimental cups per treatment per trial. Error bars for both axes are not depicted for visual clarity and to focus on trend lines. Symbols represent means, with shape corresponding to trial, and color corresponding to $\mathrm{pCO}_{2}$ (color bar at right). Lines depict linear regressions; none were significant $(P<0.05)$ 

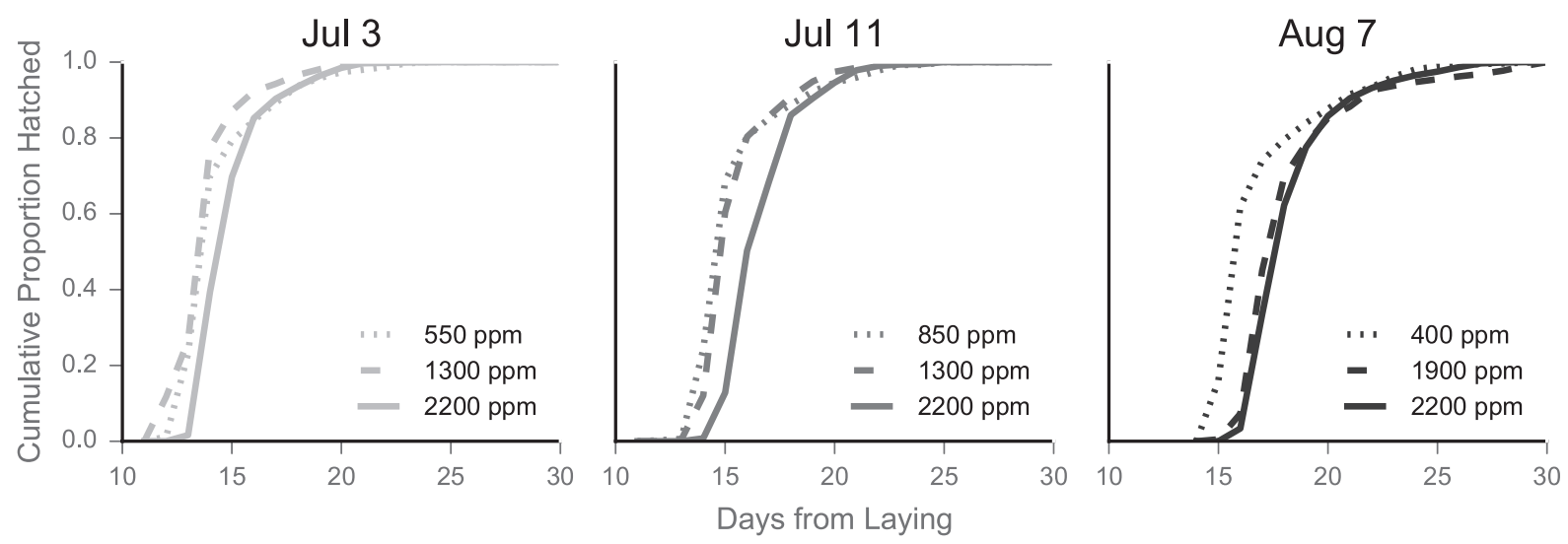

Figure 7. Hatching time curves for each $\mathrm{pCO}_{2}$ treatment. Hatching counts are plotted as the cumulative percent hatching per day to produce smooth curves. Data are plotted by trial, denoted by lay date (titles) and color; $n=3$ experimental cups (with 2 egg capsules each) per treatment per trial. Error bars/shading not depicted for visual clarity of the curves. Line patterning demarcates $\mathrm{pCO}_{2}$ treatment, with lines becoming more solid with increasing acidification 


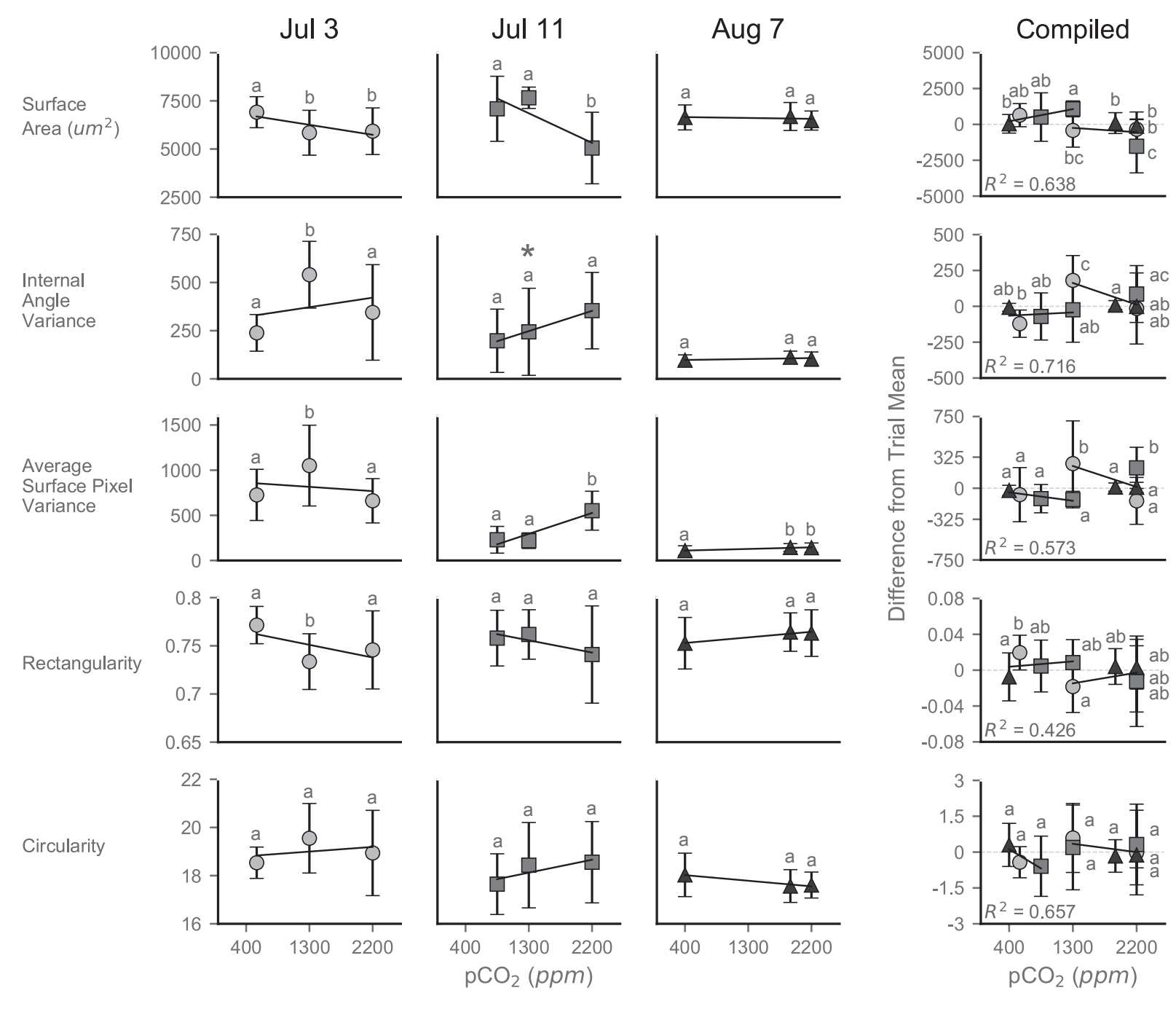

Figure 8. Statolith morphometrics across a range of $\mathrm{pCO}_{2}$ treatments. Data are presented separated by trial (demarcated by egg capsule laying date) compiled across cups and hatching days for each $\mathrm{pCO}_{2}$ treatment. The Compiled plot depicts the data from all trials normalized by taking sample values and subtracting its respective trial mean (n's in Table 1). Models of best fit from piecewise regressions are presented on compiled data with corresponding $\mathrm{R}^{2}$ values.

Symbols represent means, with shape and color corresponding to trial. Error bars represent one standard deviation. Letters demarcate statistical groupings from a Dunn's test. Lines depict linear regressions; significance is marked with an asterisk $(P<0.05)$ 


\section{Chapter 3}

\section{Ocean acidification responses in paralarval squid swimming behavior using a novel $3 \mathrm{D}$ tracking system}

This chapter was originally published as: Zakroff C, Mooney TA, Wirth C (2018) Ocean acidification responses in paralarval squid swimming behavior using a novel 3D tracking system. Hydrobiologia 808:83-106. doi: 10.1007/s10750-017-3342-9. The Supplementary Materials for this chapter can be found in Appendix B. 


\begin{abstract}
Chronic embryonic exposure to ocean acidification (OA) has been shown to degrade the aragonitic statolith of paralarval squid, Doryteuthis pealeii, a key structure for their swimming behavior. This study examined if day-of-hatching paralarval $D$. pealeii from eggs reared under chronic OA demonstrated measurable impairments to swimming activity and control. This required the development of a novel, cost-effective, and robust method for 3D motion tracking and analysis. Squid eggs were reared in $\mathrm{pCO}_{2}$ levels in a dose-dependent manner ranging from 400 - $2200 \mathrm{ppm}$. Initial 2D experiments showed paralarvae in higher acidification environments spent more time at depth. In 3D experiments, velocity, particularly positive and negative vertical velocities, significantly decreased from 400 to $1000 \mathrm{ppm} p \mathrm{CO}_{2}$, but showed non-significant decreases at higher concentrations. Activity and horizontal velocity decreased linearly with increasing $p \mathrm{CO}_{2}$, indicating a subtle impact to paralarval energetics. Patterns may have been obscured by notable individual variability in the paralarvae. Responses were also seen to vary between trials on cohort or potentially annual scales. Overall, paralarval swimming appeared resilient to OA, with effects being slight. The newly developed 3D tracking system provides a powerful and accessible method for future studies to explore similar questions in the larvae of aquatic taxa.
\end{abstract}




\section{Introduction}

Ocean acidification (OA) has emerged as a prominent threat to marine systems, with rising atmospheric $\mathrm{CO}_{2}$ concentrations decreasing ocean $\mathrm{pH}$ at rates unparalleled in geologic history (Doney et al. 2009; Honisch et al. 2012). Coastal systems are particularly susceptible due to freshwater influx and concurrent anthropogenic impacts, e.g. eutrophication, reducing the buffering capacity and increasing the $\mathrm{pH}$ variability of these waters (Gledhill et al. 2015). Nearshore marine systems provide nursery habitat to a range of ecological and economically vital species, including the longfin inshore squid, Doryteuthis pealeii, a keystone species in the Northwest Atlantic coastal trophic web and a substantial fishery (Macy III 1982; Beck et al. 2001; Jacobson 2005; Hunsicker and Essington 2008). This member of the demersal Lolignid squids is a seasonal migrator, overwintering on the continental shelf and breeding nearshore south of the Mid-Atlantic Bight, before coming north and inshore to areas like Vineyard Sound, MA, USA from late spring through early autumn for peak breeding season (Macy III and Brodziak 2001; Jacobson 2005). The squid leave mops of egg capsules tied to the seafloor, each containing embryos, 50 - 200 per capsule, which must develop under whatever conditions they are laid in, enduring environmental stress until hatching (Arnold et al. 1974; Jacobson 2005). At hatching, the paralarvae must cope both with the shock of the transition into a neritic, planktonic phase and the continued stress of their environment until they are transported by prevailing currents (Robin et al. 2014). Paralarval survivorship is naturally low, with greatest mortality occurring during the no net growth, post-hatch period while transitioning from yolk reserves to exogenous feeding (Vidal et al. 2002a; Robin et al. 2014). Sublethal physiological changes to embryonic condition, metabolism, or sensory systems imposed by environmental stressors, such as OA, could express as shifts in hatchling paralarval swimming activity and behavior. Any impairments arising during this sensitive transitional phase could be detrimental not only to individual squid success, but also to overall population structure (Byrne 2011; Robin et al. 2014).

Swimming is key to paralarval squid survival; the mantle fins are rudimentary post-hatch, therefore hatchlings rely primarily on jetting for motion, which is necessary in capturing prey and avoiding predators (Vecchione 1981). Paralarvae operate at intermediate Reynolds numbers (25 - 90), balancing between the viscous world at low speeds and a more inertial world during their high speed jets (Bartol et al. 2009b). They are also negatively buoyant: slowly, passively sinking before jetting upwards in bursts, displaying a characteristic 'hop and sink' pattern, which 
is believed to conserve energy (Haury and Weihs 1976; Staaf et al. 2014). Jetting is an energetically costly means of motion, but one that provides remarkable propulsive efficiency at the paralarval stage (Bartol et al. 2008, 2009a). During the post-hatch transitional phase, paralarvae must operate with a finite fuel reserve, the yolk, to power their jets as they avoid predation and learn to predate, but this same energy source is also tapped to mitigate stress and maintain homeostasis (Vidal et al. 2002a; Sokolova et al. 2012).

Under natural conditions, the fluid surrounding the chorions of $D$. pealeii embryos within an egg capsule reaches dramatically low $\mathrm{pH}$ (7.34) and oxygen concentrations $\left(1.9 \mu \mathrm{mol}^{-1}\right)$ prior to hatching: a potentially taxing physiological state that may be exacerbated by ocean acidification (Long et al. 2016). Both hypercapnia and decreased $\mathrm{pH}$ can elicit metabolic depression in marine ectotherms, a common stress coping response (Guppy and Withers 1999; Sokolova 2013). Depression of oxygen consumption rate has been shown to occur in the embryos and hatchling paralarvae of high- $\mathrm{CO}_{2}$ exposed eggs of the European squid, Loligo vulgaris (Rosa et al. 2014a). Cephalopods have substantial homeostatic machinery, energydependent acid-base transporters, with which they can maintain extracellular pH (Gutowska et al. 2010a). They may be capable, then, of reallocating energy across active biological processes, in order to retain their overall metabolic rate (Sokolova et al. 2012). Reduced dorsal mantle length (DML) and increased embryonic development time have been seen in D. pealeii hatchlings exposed to chronic OA, which may be indicative of such a homeostatic response (Kaplan et al. 2013). Both metabolic depression and energy budget reallocation during embryonic development could result in a subsequent reduction in hatchling paralarvae swimming activity or speed.

The statoliths (small aragonitic stones connected to sensory hair cells) are the core sensory structures for control of motion balance, and orientation in the cephalopods (Messenger 1970; Arkhipkin and Bizikov 2000). Absence of the paralarval statolith resulting from a lack of strontium in artificial seawater has been shown to cause aberrant "spinning" behaviors in several cephalopod taxa (Hanlon et al. 1989). D. pealeii paralarvae have demonstrated a reduction in statolith size and quality after exposure to high levels of $\mathrm{CO}_{2}$ during development (Kaplan et al. 2013). This present study sought to repeat and expand on the above mentioned study by recording and tracking the movement of squid paralarvae in order to examine if chronic embryonic exposure to OA caused impairments to their general swimming activity and orientation ability. 
Squid paralarvae present a distinct challenge in larval tracking with current methodologies. As small (approximate DML of $1.8 \mathrm{~mm}$, total length of $3 \mathrm{~mm}$ ), translucent organisms, squid paralarvae are well-suited to digital particle image velocimetry (DPIV) studies (Bartol et al. 2008, 2009b, a). These studies, while advantageous for dissecting the mechanics of motion and flow in many taxa, are primarily done in two-dimensions (2D) as the set up and equipment, and subsequent costs associated with requiring both a laser and high-speed camera, are substantial (Stamhuis and Videler 1995; Fuchs et al. 2004; Wheeler et al. 2013).

Early techniques of larval videography and tracking were enacted in simple, costeffective 2D systems, such as petri dishes or round aquariums, where the animal was recorded in the horizontal $x, y$ plane from a camera directly above (Wassersug and von Seckendorf Hoff 1985; Villanueva et al. 1997; Budick and O'Malley 2000). These systems were limiting for a study with squid paralarvae given the dominance of vertical motions in their swimming behavior (Staaf et al. 2014). Stereoscopic camera systems are commonly used in the field to detect accurate depth and positional information of oceanic organisms (Klimley and Brown 1983; Boisclair 1992). In lab, this method requires lighting from the front, however, which can alter the behavior of positively phototactic organisms like squid.

Using two perpendicular cameras allows for unbiased lighting while still capturing the organism in all three dimensions. Such methods can produce a clear movement track, but often require specially designed systems, either with motorized camera set ups or with uniquely shaped aquaria that limit movement range in the $y$ axis (Coughlin et al. 1992; Cachat et al. 2011a, b). We found we were limited by existing tracking software being both prohibitively expensive and, in testing, proving not to successfully function in tracking videos of the squid paralarvae. In order to observe swimming at the resolution and accuracy needed to examine our OA-driven questions, we had a clear need to develop a method of three-dimensional (3D) analysis that would not limit or coerce the motion of the organism and would produce clear, well-lit video wherein the organism could be tracked effectively by readily available software.

The aim of this study was to evaluate the potential effects of ocean acidification on posthatch paralarval squid, D. pealeii, swimming behavior using newly-hatched paralarvae reared under a range of $\mathrm{CO}_{2}$ concentrations (and thus a range of $\mathrm{pH}$ treatments). Across their range, and dependent on season, adult D. pealeii can be found in depths ranging from $1-400 \mathrm{~m}$, temperatures ranging from $4-28{ }^{\circ} \mathrm{C}$, and salinities ranging from 30-37 ppt. Juvenile and adult $D$. 
pealeii have been found in the Hudson-Raritan estuary, a system with much greater temperature, salinity, and $\mathrm{pH}$ variation than the coastal shelf, which may indicate these life stages are capable of at least acute exposure to a wide range of environmental conditions (Jacobson 2005). Comprehensive measurements of in situ environmental $\mathrm{pH}$ in $D$. pealeii habitat across its range have not been performed to our knowledge, but ranges of mean shelf $\mathrm{pH}_{\text {total }}(20)$ of $7.85-8.05$ and $f \mathrm{CO}_{2}(20)$ of $400-700 \mathrm{ppm}$ were reported from North Carolina to New Hampshire from a coastal carbon cruise conducted in summer (July/August) (Wang et al. 2013). Little is documented about the ecology or environmental exposures of the paralarval life phase of squid, but rearing experiments with Loliginids indicate high water quality and a recommended $\mathrm{pH}>8.0$ are best for their survival (Hanlon et al. 1983; Vidal et al. 2002b).

Our study focused on specimens from and comparisons to the Vineyard Sound, MA system where $D$. pealeii eggs are laid every summer. This work encompassed a range of $\mathrm{CO}_{2}$ levels between current ambient ( 400 ppm) and the elevated treatment used in Kaplan et al. (2013). The high treatment, $2200 \mathrm{ppm}$, is predicted for 2300 based on IPCC IS92a, but is naturally found in the very extreme inshore estuary conditions of Vineyard sound (Caldeira and Wickett 2003; McCorkle et al. 2012). The intent was to examine the physiological scope and sensitivity of hatchling paralarval swimming. We hypothesized, based on initial work by Kaplan et al. (2013), that activity levels, speed, and control of orientation would be impaired in OAexposed paralarvae due to impairments to their physiological and sensory systems. The question posed required a robust visualization of the energetics and kinematics of paralarval swimming, which required the development of a novel, simple, and feasible method of 3D paralarval tracking and analysis that we present here alongside the experimental data.

\section{Materials and Methods}

Squid Collection and Husbandry

Experiments were conducted at the Environmental Systems Laboratory (ESL) at the Woods Hole Oceanographic Institution, Woods Hole, MA, USA from June-August 2013, MayOctober 2014, and May-June 2015. This timing corresponds with the peak breeding season of the Atlantic Longfin squid, Doryteuthis pealeii, in the nearshore of New England (Jacobson 2005). Although the full breadth and physical properties of D. pealeii egg habitat is not well described, eggs in New England waters are typically found at depths less than $50 \mathrm{~m}$, with temperature and 
salinity ranges of $10-23^{\circ} \mathrm{C}$, and 30 - 32 ppt, respectively (McMahon and Summers 1971; Jacobson 2005; Shashar and Hanlon 2013). Squid were collected during trawls in Vineyard Sound at 10-30 meters depth by the Marine Biological Laboratory (MBL). Sea surface temperatures in Vineyard Sound, MA, USA ranged from $8.4-25.8^{\circ} \mathrm{C}$ with a mean of $19.4^{\circ} \mathrm{C}$ from May - October (compiled for 2013 - 2015 from NOAA Station BZBM3). Bottle samples (n =5) were taken in Vineyard Sound at $20 \mathrm{~m}$ depth (processed for Alk/DIC using VINDTA) in the morning once every two weeks from late July - late September 2014 off of the MBL squid trawler at the site of capture, along with accompanying CTD casts (CastAway CTD, SonTek, San Diego, USA). These data show a temperature range of $17.4-19.6^{\circ} \mathrm{C}$, salinity range of 31.3$32.5 \mathrm{psu}$, total alkalinity range of 2148.1 - $2195.2 \mu \mathrm{mol} / \mathrm{kg}$, DIC range of 1962.7 - $2038.2 \mu \mathrm{mol}$ $/ \mathrm{kg}, \mathrm{pH}_{\text {total }}$ range of $7.96-8.00$, and $\mathrm{pCO}_{2}$ range of $439.9-486.5 \mathrm{ppm}$, but are limited in scope in reference to the whole breeding season.

Intact, adult squid (mid-sized, 20-25 cm DML without fin tears/skin lesions) were handselected from the trawl catch at the MBL's Marine Resources Center dock. Individuals were gently placed in seawater-filled coolers and transported by car to the ESL immediately after the ship's return ( $<6$ hours post-capture), and transferred to the ESL holding aquaria. All transport activity was performed as carefully and expediently as possible to minimize stress to the breeding adults, but overall capture and transit stress was unavoidable. Eighteen squid were selected for breeding; reproductively active females (differentiated by their bright orange accessory nidamental gland) and males, displaying visible and dense sperm packets, were selected in a 2:1 female:male ratio to enhance breeding probability.

Upon arrival at the ESL, squid were split equally between two holding tanks $(120 \mathrm{~cm}$ diameter, $70 \mathrm{~cm}$ depth), maintaining the $2: 1$ gender ratio. Holding tanks were flow-through, using water pumped directly from Vineyard Sound (approximately 100 yards offshore of the ESL) that had been sand-filtered and cooled to $15^{\circ} \mathrm{C}$ (Salinity $\left.=33 \mathrm{psu}, \mathrm{pH}_{\mathrm{nbs}}=7.96\right)$, and continuously bubbled with air. This temperature was within the range naturally experienced by the adults during the breeding season and reduced thermal and metabolic stress on the adults, as well as the incidence of infighting and cannibalism, compared to if they were housed at ambient temperatures. Squid were fed once per day with locally captured killifish, Fundulus heteroclitus, gathered from Salt Pond, Woods Hole, MA. Upon discovery, mops of egg capsules were transferred into a bucket of water from the adult tank and carried to the room containing the 
ocean acidification system where they were hand sorted into the experimental cups. Only good quality egg capsules, those that were long and finger-like with an orange tinge and laid in neat mops, were chosen; egg capsules contained between 90 - 300 eggs, which is expected for this species (Arnold et al. 1974; Maxwell and Hanlon 2000). Adult squid were maintained in the tanks at the ESL until they died following breeding.

\section{Ocean Acidification System}

In brief, for each trial, D. pealeii egg capsules collected from the squid aquaria were randomly sorted into flow-through cups (1-liter PET food service containers [Solo Foodservice, Lake Forest, IL]) filled with seawater delivered via drip lines from upstream equilibration chambers. These chambers were bubbled with $\mathrm{CO}_{2}$ and air to maintain respective $\mathrm{CO}_{2}$ concentrations (ranging from $400 \mathrm{ppm}$ to $2200 \mathrm{ppm}$ ). All rearing cups were contained within a $20^{\circ} \mathrm{C}$ water bath under a 14:10 hour light:dark cycle. No temperature acclimation was conducted for the eggs, as this level of temperature shift did not appear to notably impact embryonic development or survival, or paralarval viability. This temperature and light regime approximated the average values in Vineyard Sound $\left(19.4^{\circ} \mathrm{C}\right)$ across the breeding season, late April to early October. A rearing temperature of $20^{\circ} \mathrm{C}$ was chosen as it reflected natural conditions, to replicate the conditions in the previous work by Kaplan et al. (2013), and because temperature controls the development time of $D$. pealeii, and so resulted in a consistent 14 day time to hatching at the control $p \mathrm{CO}_{2}$ (McMahon and Summers 1971; Zeidberg et al. 2011). There were three cups containing two egg capsules each and one chemical control cup per treatment per trial. Flow rates to the cups were approximately 201 day $^{-1}$, which prevented waste accumulation. Water quality of the experimental cups was monitored using a $\mathrm{pH}$ probe (Orion Star ${ }^{\mathrm{TM}} \mathrm{A} 329$, Thermo Fisher Scientific Inc., Waltham, MA, USA) every three days, while alkalinity, salinity, and spectrometric $\mathrm{pH}$ readings were taken weekly in order to calculate $p \mathrm{CO}_{2}$ with CO2SYS (Table 1) (a full OA system description, experimental procedure, and $\mathrm{CO}_{2}$ monitoring methods can be found in the Supplementary Materials).

\section{Paralarvae Sampling}

Squid embryos were allowed to develop undisturbed in the OA system. Upon hatching, paralarvae from each cup were subsampled for a range of experiments, including behavioral 
videography. At the end of each hatching day, all paralarvae were removed from the system and preserved (anesthetized with $7.5 \% \mathrm{w} / \mathrm{v} \mathrm{MgCl}_{2}$ mixed with equal part seawater and preserved in $70 \%$ ethanol) ensuring that all hatchlings used were less than 24 hours old. Over the first 3 - 6 days of hatching, 10 - 20 individual paralarvae per cup per treatment were collected for behavioral videos, in order to obtain multiple analyzable videos per treatment per trial. Paralarvae were haphazardly selected from the cups, avoiding those that exhibited a constant spinning behavior, which has been described both as an aberrant effect of aquarium-rearing and as a potential stereotyped predator defense (Hanlon et al. 1989; York and Bartol 2016). Tests in 2013 demonstrated there was no difference in this behavior across $\mathrm{CO}_{2}$ treatments (ANOVA, $\mathrm{F}_{5}$, $\left.{ }_{21}=2.31, \mathrm{p}=0.0805\right)$. As there was only one arena/camera set up, videography was done for one experimental cup at a time. Per each cup, the arena was filled with water from the control cup for that treatment. Individual paralarvae $(n=10$ - 15) from the experimental cup were transferred using a plastic pipet and kept within a 24 well plate (Falcon ${ }^{\circledR}$ Brand $2.0 \mathrm{~cm}^{2}$ well area, $3.5 \mathrm{~mL}$ well volume, Corning Inc., Corning, NY, USA) filled with water from the same cup, one paralarva per well, until filming occurred. A filming period for one cup took at most an hour ([1 minute acclimation +2 minutes recording +1 minute removal and reset $] * 10-15$ paralarvae $=$ 40 - 60 minutes per cup). It was assumed, although not tested, that water quality in the well plate and arena was that of the experimental treatment sampled and did not notably change over the brief filming period. The arena and well plate were refilled for each filming period and treatments were selected from in a rotation, so as to not bias sampling by time of day. Overall more videos were recorded than were analyzed in both the 2D and 3D systems for all analyses, as only videos where the paralarvae was visible, away from the corners, exhibiting normal swimming/responses, and, in the case of the 3D metrics, trackable could be used (Table 1)

\section{Swimming Behavior Experiments}

Over the course of developing a viable 3D system, two different arenas were used resulting in two separate swimming behavior experiments. Experiment 1 consisted of Trials 1, 2 and 4 of 2013 and Trial 1 of 2014 (Table 1). Trials were run in a tall, rectangular arena that constrained movement in the $y$ axis, but only $2 \mathrm{D}$ vertical swimming data could be tracked and analyzed from this system. Implemented in Experiment 2 (Trials 2, 3, and 4 in 2014 and in Trial 1 of 2015; Table 1), the cubic arena and model system allowed for full 3D tracking and analysis. 
Wall effects were considered for both experiments, however the viscous effects of walls at low Reynolds numbers should not impact paralarvae given their size and speed (Vogel 1981). Damage caused by wall impacts is a common source of mortality among aquarium housed squid, and it is best to make the walls visible to help avoid this, however this would have obscured recording and lighting (Summers et al., 1974). Many paralarvae interacted with the walls during recordings, but this did not appear to cause harm or behavioral shifts, although paralarvae were not subsequently checked for dermal abrasions. Increasing the available swimming volume from the $2 \mathrm{D}$ to the $3 \mathrm{D}$ arena was intended to reduce potential arena effects, although overall arena size was limited by available space (e.g. camera viewing scope within the covered light box).

In order to determine acclimation time, 10-minute recordings of individual paralarvae ( 9 useable videos analyzed of 20 videos taken), sampled from all treatments, placed into the arena were conducted. Paralarval activity, described by jetting or active mantle pulsation, showed no significant differences across 1-minute bins of the ten-minute observation period (KruskalWallis, $p=0.9284)$. We do not claim that the paralarvae became accustomed to the arena, however there were no significant changes to their behavior within a reasonable recording timeframe (Supplementary Materials, Figure S1). An acclimation time of 1 minute was therefore used for both experiments, in order to maximize the sample size of videos taken (Table 1).

\section{Experiment 1: 2D Swimming Behavior}

The trials of the first experiment took place during the summer of 2013 and in May of 2014. Experiment 1 used a preliminary 2D filming arena constructed from $500 \mathrm{~mL}$ tissue culture flasks (Corning Inc., Corning, NY, USA) by removing the top, capped portion, creating a standing container with internal dimensions of $10.6 \mathrm{~cm}$ width, $3.2 \mathrm{~cm}$ depth, and $14.0 \mathrm{~cm}$ height ( $x, y$, and $z$ axes) (Figure S2a). All plastic containers were soaked for 24 hours in seawater and DI-water rinsed prior to use. Black card stock was attached to the back of the arena for contrast between the translucent organism and the background, allowing for better paralarval tracking. $400 \mathrm{~mL}$ of water from the corresponding $\mathrm{CO}_{2}$ level was added to the chamber, corresponding to a $10.5 \mathrm{~cm} \times 3.2 \mathrm{~cm} \times 11.4 \mathrm{~cm}(x, y$, and $z)$ volume. This provided a large area, compared to the organism's size (approximately $3 \mathrm{~mm}$ ), in the $x$ and $z$ axes while constraining movement in the $y$ axis. This chamber was placed within a photobox $\left(76.2 \mathrm{~cm}^{3}, \mathrm{~B} \& \mathrm{H}\right.$ Foto \& Electronics Corp, NY), which was covered with black tarp to block ambient light. Two LED panels with diffuser 
plates were placed on either side of the chamber to create equal lighting from both directions. The strong photopositive response by paralarvae required lights be placed precisely and set to equal intensities to exclude directional bias. Two HD video cameras were placed inside the photobox at a $90^{\circ}$ angle to the experimental chamber, one above (Sony HDR-XR550V) fastened to a wooden frame and one in front (Sony HDR-CX 580V). Only information from the front camera was used in later analyses due to poor larval visibility for tracking in footage from the top-mounted camera. Videos were recorded at 29.97 frames per second.

For each video, an individual paralarva was pipetted from the tray directly into the center of the chamber. After the one-minute acclimation period, swimming behavior was recorded for one minute. Paralarvae were removed from the chamber using a pipet and were anesthetized with $7.5 \% \mathrm{w} / \mathrm{v} \mathrm{MgCl}_{2}$ mixed with equal part seawater before being preserved in $70 \%$ ethanol.

\section{Experiment 1 Data Analysis}

Individual paralarvae videos were tracked using Tracker, marking the eyes of the paralarvae, which were the most distinct and trackable feature (Open Source Physics, comPADRE Digital Library). Variability in the 2D system's video quality, due to issues of light reflection and clarity, and individual paralarval trackability (many paralarvae stayed near the walls, entrained in the meniscus, or expressed the aforementioned spinning behaviors, which made videos unusable) resulted in uneven sampling amongst the $2 \mathrm{D}$ data (total $\mathrm{n}=394$, Table 1 ). Organisms were tracked using the autotracker function when video quality allowed this for capability, but this function was corrected with manual tracking as needed. Positional data $(x \& z$ coordinates) were produced for each frame within the 60 -second interval, totaling 1799 points per recording. The $z$ axis of the arena was divided into equal thirds of $4.73 \mathrm{~cm}$ and positional data were sorted into these depth bins for the top, middle, and bottom of the container using Excel (Excel for Mac 2011, Microsoft Corp., Redmond, WA, USA). The number of frames per bin, directly proportional to time spent in each depth section, was calculated for each individual and compared across $\mathrm{CO}_{2}$ treatment groups.

\section{Experiment 2: 3D Swimming Behavior}

The trials of the primary experiment using the developed 3D analysis system took place in the summer of 2014 and May of 2015. The recording arena for the 3D filming consisted of the 
bottom portion of a plastic display box $(10.2 \mathrm{~cm}$ x $10.2 \mathrm{~cm}$ x $18.4 \mathrm{~cm}$, Amac Plastic Products Corp., Petaluma, CA, USA) (Figure S2b). Black card stock was attached to the back of the container in the $x, z$ plane and black cotton cloth laid on the bottom of the container in the $x, y$ plane, again to contrast translucent paralarvae. In the 2D arena, excessive light reflection could obscure paralarvae near the walls and a strong meniscus could entrain paralarvae to the surficial corners, but the greater area, and increased wall clarity of the $3 \mathrm{D}$ arena prevented these issues.

The 3D filming arena was set up in the same manner as the $2 \mathrm{D}$ arena within the photobox. The camera lenses were each aligned to the centroid of the chamber for their respective viewing planes (Figure 1, A \& B). Each camera was connected to its own monitor outside of the photobox so that paralarval swimming could be observed during the trials.

The chamber bottom measured $92.16 \mathrm{~cm}^{2}$ inside $(9.6 \mathrm{~cm} \times 9.6 \mathrm{~cm})$; therefore, the chamber was filled to $9.6 \mathrm{~cm}$ depth with water of the appropriate $\mathrm{CO}_{2}$ concentration to create an $884.7 \mathrm{~cm}^{3}$ cube to contain the organism (the model system requires a cubic water volume, as outlined below, but the size can be changed). Individual paralarvae were pipetted from a holding tray directly into the center of the filming arena and allowed to acclimate for one minute. Swimming behavior was then recorded for two minutes; increased from the previous 2D experiments due to the improved video quality and tracking output of the 3D system as well as the need for more path data for the more complex 3D metrics. The two minute period was concluded by flashing a laser pointer into the filming chamber, which provided a synchronization point for the top and side videos. Paralarvae were then removed from the chamber, anesthetized, and preserved in $70 \%$ ethanol.

\section{Experiment 2 Data Analysis}

Videos from the top and side cameras were synched using the laser flash and cut to twominute clips for each paralarva. Videos were tracked using Tracker, using the eyes of the paralarvae as the tracking feature, as with the $2 \mathrm{D}$ experiment. Although the $3 \mathrm{D}$ arena did not diminish video quality as the 2D arena did, a large number of videos could still not be used, due to paralarvae staying at walls and corners, or spinning (total tracked video $\mathrm{n}=157$, Table 1). Positional data were produced for each frame in both viewing planes, resulting in 3,598 points for each recording plane $(x, z)$ and $(x, y)$. Tracker functioned with the origin point for the calibrating axes being set based on the orientation of the camera. Thus prior to correction for 3D, 
the positional data were all transformed into the same $0,0,0$ axes frame set at the bottom left corner of the front plane of the filming arena using Excel.

To merge the two separate $2 \mathrm{D}$ positional datasets into a $3 \mathrm{D}$ dataset, the filming arena was modeled as two series of diminishing planes, "side" and "top" (Figure 1, C \& D). In any given video frame, the front plane of the chamber had the true dimensions of the arena, $92.16 \mathrm{~cm}^{2}$. Along the axis perpendicular to the axes of the video frame, the planes diminish in the image due to the vanishing point effect, up unto the back of the arena. The value of the length of the side of the cube was known $(Q=9.6 \mathrm{~cm})$. The organism was in some plane along the perpendicular axis, so its position within its plane in the image is proportional to its true position in a $9.6 \mathrm{~cm}$-sided square, such that

$$
\frac{Q_{S}}{Q}=\frac{x_{i s}}{x}
$$

$$
\frac{Q_{S}}{Q}=\frac{z_{i s}}{z}
$$

$$
\frac{Q_{t}}{Q}=\frac{x_{i t}}{x}
$$

$$
\frac{Q_{t}}{Q}=\frac{y_{i t}}{y}
$$

where $Q_{s}$ and $Q_{t}$ are the lengths of the plane the organism is in within the image and $x_{i s}, z_{i s}, x_{i t}$ and $y_{i t}$ are the positional coordinates of the organism within that plane, in the side and top, respectively.

The positional values of the organism were measureable within the coordinate axes of the front plane using Tracker: $x_{s}$ and $z_{s}$ for the side, and $x_{t}$ and $y_{t}$ for the top. The positional values of the organism in its image plane were modeled as the difference between these measured coordinates and the side of the right triangle formed between the front plane and the organism's image plane (Figure 1, C \& D):

$$
\begin{aligned}
& x_{i s}=x_{s}-q_{i s} / \sqrt{2} \\
& z_{i s}=z_{s}-q_{i s} / \sqrt{2} \\
& x_{i t}=x_{t}-q_{i t} / \sqrt{2} \\
& y_{i t}=y_{t}-q_{i t} / \sqrt{2}
\end{aligned}
$$


where $q_{i s}$ and $q_{i t}$ are the hypotenuse of the right triangles for the side and top, respectively. The hypotenuse divided by the square root of two provides a measure of distance between the front plane and the organism's image plane. It follows then that $Q_{s}$ and $Q_{t}$ are different to $Q$ by twice this measure:

$$
\begin{aligned}
& Q_{s}=Q-2 *\left(q_{i s} / \sqrt{2}\right) \\
& Q_{t}=Q-2 *\left(q_{i t} / \sqrt{2}\right)
\end{aligned}
$$

The length of the side of the backmost plane was measureable within the film image for both the side and top camera ( $q_{b s}$ and $q_{b t}$, respectively). The ratios between these values and the length of the filming arena are equal to the ratios between the distances between planes, $q_{i s}$ and $q_{i t}$, and the true distance of the image plane along the perpendicular axis, $y$ and $z$, respectively:

$$
\begin{aligned}
& \frac{q_{b s}}{Q}=\frac{q_{i s}}{y} \\
& \frac{q_{b t}}{Q}=\frac{q_{i t}}{z}
\end{aligned}
$$

This results in an over-constrained system. Datasets for individual paralarva with all measured values were run through a MATLAB (R2016b, MathWorks, Inc., Natick, MA, USA) custom-built script (in the Supplementary Materials and at https://github.com/czakroff/3DSwimming-Behavior) which calculated the $x, y$, and $z$ values for the system by using least sum of squares to determine the values for each frame that result in the least error. These data were then run in a separate MATLAB custom-built script (in the Supplementary Materials and at https://github.com/czakroff/3D-Swimming-Behavior) to visualize paralarval swimming tracks and calculate a range of 3D metrics, including total distance traveled $(\mathrm{cm}), 3 \mathrm{D}$ velocity $\left(\mathrm{cm} \mathrm{s}^{-1}\right)$, vertical and horizontal velocities $\left(\mathrm{cm} \mathrm{s}^{-1}\right)$, and volume $\left(\mathrm{cm}^{3}\right)$ transited for each individual paralarva. All video measurements were taken and all subsequent analyses using the model system and codes were run using centimeters. However, all results have been shifted into millimeters for better readability in publication. 3D metrics were further analyzed in ten-second time bins across the 120 -second recording period for each individual paralarvae, to examine individual variability and assess if paralarvae across treatments retained consistent overall behavior patterns while in the 3D arena.

Since squid paralarvae swim in a characteristic cycle of vertical jetting and sinking, average vertical velocity canceled out to zero. Therefore, vertical velocity was subdivided into average 
positive vertical velocity, representative of upward jets, and negative vertical velocity, representative of sinking and uncommon, but possible, downward jets. Average jet velocity was calculated by measuring the magnitude of the peaks, above a threshold of $0.5 \mathrm{~cm} \mathrm{~s}^{-1}$ (based on visual assessment of the data and on a reported range of jetting velocities in Bartol et al., 2009a), of the $3 \mathrm{D}$ velocity data across the two-minute recording period for each paralarvae. Jetting rate was likewise calculated by enumerating these velocity peaks for each paralarvae.

Turning angles for each paralarva's path were calculated between sequential motion vectors in the $x, y$ plane at a resolution of thirty frames of video, or approximately one second of motion. Tortuosity, a metric of path convolution defined as the ratio of the length of an animal's path to the distance between the start and end points of that path, was calculated for each paralarva on path segments of thirty frames of video continuously along the entire path (Benoit-Bird and Gilly 2012). The one-second resolution for these metrics was chosen to analyze individual paralarval paths on a reasonable temporal scale and reduce small-scale motion noise.

While thresholds of video quality and organism visibility limited the total number of videos useable in the 3D tracking analysis, many more videos were useable for simpler analyses. In some cases the side video was trackable or could be visually assessed while the top was not, so a random subset $(n=282$, Table 1$)$ of $3 \mathrm{D}$ videos taken was analyzed for time spent in depth bins, as in the 2D experiment. A separate randomly selected subset of approximately ten of the twominute paralarva swimming videos taken was analyzed per treatment per trial $(\mathrm{n}=167$, Table 1$)$ for general ethography. Sections of each video were coded as either active, defined as a paralarvae jetting or pulsing its mantle, or inactive, defined as sinking, non-motile, and nonpulsing. Two observers each independently coded the same subset of 10 individual paralarvae to establish consistency in the definitions and then separately coded different subsets of the full dataset. Ethographic observations of activity were compiled as percent time active (converted from number of frames using the video frame rate) for the entire two-minute recording period for each individual paralarva.

\section{Statistics}

Statistical analyses were run in MATLAB, Python (3.5.2), and Excel. Normality was tested for using the Shapiro-Wilk test $(\alpha=0.05)$ and by examining quantile plots of the data for each factor, both within each treatment and as a whole. Datasets that were normally distributed 
were tested for differences among $\mathrm{CO}_{2}$ treatments with a single factor ANOVA, while nonparametric data were tested with Kruskal-Wallis $(\mathrm{KW})$ tests. Any significant $(\mathrm{p}<0.05) \mathrm{KW}$ test was subsequently run through a Dunn's posthoc test to determine which groups were significantly different from each other. Linear trend lines of the medians for each factor across $\mathrm{CO}_{2}$ treatments were plotted in order to examine goodness of fit to the trend. Variability was examined through calculation of the variance, comparable within a metric, and the coefficient of variation $(\mathrm{CV})$, which is comparable across metrics. All normally distributed statistics are reported as means \pm 1 standard deviation $(\mathrm{SD})$ while nonparametric statistics are reported as medians and interquartile range (IQR).

\section{Results}

Water Quality

No significant differences (Kruskal-Wallis, $\mathrm{p}>0.05$ for $\mathrm{pH}_{\text {total }}$ and $p \mathrm{CO}_{2}$ for all trials) in water quality were seen between experimental cups within a treatment, so values are compiled and reported by treatment (Table 1). Temperature and salinity were stable across the duration of a trial, but varied slightly between trials, most likely due to local environmental variability. $p \mathrm{CO}_{2}$ equilibrations were harder to control at higher concentrations, likely due to variability in the alkalinity and flow rate of ESL water (potentially due the expanded system, longer time frame, and increased demand in 2014 \& 2015), as input gas concentrations and pressure were maintained throughout experiments and $\mathrm{pH}_{\text {total }}$ remained consistent within trials (Table 1). Results are grouped and reported by input gas concentration for concision, but it should be noted that the 2200 ppm group encompasses a range from 1750 - 2400 ppm in calculated $p \mathrm{CO}_{2}$ values.

\section{Experiment 1: 2D Depth}

Squid paralarvae showed a slight, but significant $(\mathrm{KW}, \mathrm{p}<0.001)$ difference in proportion of time spent in the top depth bin between $\mathrm{CO}_{2}$ treatment groups in 2013 (Figure 2A), but no significant response in $2014(\mathrm{KW}, \mathrm{p}=0.078)$ (Figure 2B). In the compiled dataset, within the top depth bin, the 400 and 1300 ppm $\mathrm{CO}_{2}$ treatment groups were found to be different from the 1900 and 2200 ppm treatment groups, with less time spent at surface in the higher $\mathrm{CO}_{2}$ treatments (Table 2). Similarly, tests for proportion time spent in the middle and bottom depth bins also showed differences between treatment groups $\left(\mathrm{KW}, \mathrm{p}_{\text {mid }}=0.001, \mathrm{p}_{\text {bottom }}=0.002\right)$, 
wherein the $1300 \mathrm{ppm}$ treatment group was distinct from the 1900 and $2200 \mathrm{ppm}$ treatment groups, with more time spent in the middle and bottom depth bins in the higher $\mathrm{CO}_{2}$ treatments (Table 2). This reflects the extremely low variance in the $1300 \mathrm{ppm}$ treatment group compared to all other treatments in all depth bins $\left(\sigma^{2} \leq 0.001\right.$, Table 3$)$. Variance did not significantly increase with $\mathrm{CO}_{2}$ level in the top or bottom depth bin, but showed an increasing trend with increasing $\mathrm{CO}_{2}$ level in the middle depth bin $\left(\mathrm{R}^{2}=0.609\right.$, Table 3$)$. Despite notable individual variability across treatments and interannual variability in response, the experiment indicated that squid paralarvae spent less time at the surface in $\mathrm{CO}_{2}$ treatments of 1900 and $2200 \mathrm{ppm}$ overall (Figure 2C).

\section{Experiment 2: 3D Metrics}

In the 3D system, paralarvae showed no difference in the proportion of time spent in any depth bin across $\mathrm{CO}_{2}$ treatments $\left(\mathrm{KW}, \mathrm{p}_{\text {top }}=0.1094, \mathrm{p}_{\text {mid }}=0.0568, \mathrm{p}_{\text {bottom }}=0.0694\right.$, Table 4$)$ in all trials of both 2014 and 2015, nor were there any notable trends in the variance for this metric (Table 5). The proportion of time spent in the top depth bin showed a weak, non-significant decrease with increasing $\mathrm{pCO}_{2}\left(\mathrm{R}^{2}=0.6455\right)$ and proportions of time spent in the mid and bottom depth bins showed corresponding, non-significant increasing trends with increasing acidification (Table 4).

Of the 3D metrics measured for the paralarvae, total distance $(\mathrm{KW}, \mathrm{p}=0.0342)$, average velocity $(\mathrm{KW}, \mathrm{p}=0.0354)$, average positive vertical velocity $(\mathrm{KW}, \mathrm{p}=0.0126)$, and average negative vertical velocity $(\mathrm{KW}, \mathrm{p}=0.0028)$ showed significant effects of $\mathrm{CO}_{2}$ treatment $($ Table 4). Dunn's posthoc test revealed the difference to be between the 400 and $1000 \mathrm{ppm}$ groups for all of these metrics (Table 6). Average velocity was slightly higher in the $400 \mathrm{ppm}$ treatment level, $9.4 \mathrm{~mm} \mathrm{~s}^{-1}$ (8.2 - 11.7), compared to the other $\mathrm{CO}_{2}$ treatments: $8.0 \mathrm{~mm} \mathrm{~s}^{-1}(7.1-9.3), 8.3$ $\mathrm{mm} \mathrm{s}^{-1}$ (7.6 - 9.9), and $8.2 \mathrm{~mm} \mathrm{~s}^{-1}$ (7.0 - 9.7) for 1000, 1600, and $2200 \mathrm{ppm}$, respectively (Table 4). A linear fit $\left(\mathrm{R}^{2}=0.4591\right)$ of the median average velocities across treatments demonstrates this potential decreasing trend with increasing $p \mathrm{CO}_{2}$. The step-wise nature of this trend is reflected in the average positive and negative vertical velocity values (Table 4), while the horizontal component of the velocity showed a significant fit to a linear decreasing trend $\left(\mathrm{R}^{2}=\right.$ $0.9798, p=0.0101)$ rather than any significant differences between treatments $(K W, p=0.1945)$. Percent time active, as assessed in the ethological work, also followed a significant decreasing 
linear trend $\left(\mathrm{R}^{2}=0.9798, \mathrm{p}=0.0077\right)$, without significant differences between groups $(\mathrm{KW}, \mathrm{p}=$ $0.242)$.

Analyzed individuals exhibited the expected, stereotypical motion of hatchling paralarvae, swimming with repeated, and dominantly vertical, jetting motions (Figure 3 ). Average jet velocity was highest in the 400 ppm treatment, $17.6 \mathrm{~mm} \mathrm{~s}^{-1}$ (14.2 - 19.6), and notably, but not significantly $(\mathrm{KW}, \mathrm{p}=0.1192)$ lower, in the $1000,14.9 \mathrm{~mm} \mathrm{~s}^{-1}(13.1-16.5)$, $1600,14.7 \mathrm{~mm} \mathrm{~s}^{-1}$ (13.0 - 16.9), and $2200 \mathrm{ppm}, 15.1 \mathrm{~mm} \mathrm{~s}^{-1}$ (12.5 - 17.9) treatments. Peak velocity was highest in the $400 \mathrm{ppm}$ treatment, $139.6 \mathrm{~mm} \mathrm{~s}^{-1}(99.9-206.9)$, and the $1000 \mathrm{ppm}$ treatment, $138.2 \mathrm{~mm} \mathrm{~s}^{-1}\left(78.8\right.$ - 228.7), notably lower in the $1600 \mathrm{ppm}, 108.1 \mathrm{~mm} \mathrm{~s}^{-1}(86.4-$ 163.3), and $2200 \mathrm{ppm}$ treatments, $123.9 \mathrm{~mm} \mathrm{~s}^{-1}$ (82.1 - 187.5), showing a weak decreasing linear trend $\left(\mathrm{R}^{2}=0.4578\right)$, but no statistical significance $(\mathrm{KW}, \mathrm{p}=0.4378)$. Vertical and horizontal peak velocities also show this pattern of more step-wise and weakly linear, non-significant decrease from $400 \mathrm{ppm}$ (Table 4). Jetting rate, on the other hand, showed similar values between 400 ppm, 2.73 Jets s$^{-1}(2.51-3.02), 1000$ ppm, 2.70 Jets s$^{-1}(2.51-2.89)$, and 1600 ppm, 2.72 Jets $\mathrm{s}^{-1}(2.47-2.85)$, and only decreased slightly at $2200 \mathrm{ppm}, 2.63 \mathrm{Jets} \mathrm{s}^{-1}(2.38-2.81)(\mathrm{KW}, \mathrm{p}=$ $0.3436)$.

Three-dimensional polygons of volume transited, tortuosity paths, and turning angle distributions were also determined (Figure 4). Volume traveled by the paralarvae during the swimming recording was notably, but not significantly $(\mathrm{KW}, \mathrm{p}=0.7416)$ lower in the $2200 \mathrm{ppm}$ treatment, $46,406 \mathrm{~mm}^{3}(22,078-118,883)$, compared to the other treatments, $53,786 \mathrm{~mm}^{3}$ $\left(20,550\right.$ - 95,664), 65,076 $\mathrm{mm}^{3}(33,647-125,970)$, and $53,315 \mathrm{~mm}^{3}(30,734-103,950)$ for 400 , 1000 , and 1600 ppm respectively, and demonstrated a weakly decreasing linear trend $\left(\mathrm{R}^{2}=\right.$ 0.4334). Variance was high for volume transited across treatments $\left(\sigma^{2}\right.$ range $=2.86 \times 10^{9}-7.71$ $\times 10^{9}$, overall $\left.\mathrm{CV}=0.918\right)$ and appeared to increase with increasing $\mathrm{CO}_{2}\left(\mathrm{R}^{2}=0.8710\right.$, Table 5).

Average turning angle was highest in the 400 ppm treatment group, $56.53^{\circ}\left(48.84^{\circ}\right.$ $\left.64.54^{\circ}\right)$, whereas values were similar amongst the other treatments: $53.49^{\circ}\left(48.45^{\circ}-62.04^{\circ}\right)$, $52.05^{\circ}\left(45.92^{\circ}-60.00^{\circ}\right)$, and $52.30^{\circ}\left(43.64^{\circ}-63.71^{\circ}\right)$ for 1000,1600 , and $2200 \mathrm{ppm}$, respectively, resulting from a slightly higher occurrence of reversals in the $400 \mathrm{ppm}$ group. Similar to other metrics, average turning angle was not statistically significant ( $\mathrm{KW}, \mathrm{p}=0.4334)$, but still showed a slightly decreasing linear trend $\left(\mathrm{R}^{2}=0.7863\right)$. No apparent impacts of acidification on paralarval control of orientation were observed. Distributions of the turning 
angles from the entire paralarval path did not vary between treatments, except in the $120^{\circ}-130^{\circ}$ bin where turns were rare (Average frequency of turns, $120-130^{\circ}$, all treatments $<0.025$; $\mathrm{KW}_{120-130}, \mathrm{p}=0.013 ; \mathrm{KW}, \mathrm{p}>0.05$ for all other turning angle bins). All experimental paralarvae demonstrated primarily forward and reverse motions, due to the dominance of jetting, and a slightly higher frequency of shallow $\left(10-40^{\circ}\right)$ forward turns along their swimming paths.

Average tortuosity did not vary notably between $\mathrm{CO}_{2}$ treatments $(\mathrm{KW}, \mathrm{p}=0.6730)$. It was highest in the $2200 \mathrm{ppm}$ treatment, 3.63 (3.07 - 5.19), and lowest in the $1600 \mathrm{ppm}$ treatment, 3.39 (2.92 - 4.51), showing a weakly increasing trend with increasing $\mathrm{CO}_{2}\left(\mathrm{R}^{2}=0.4065\right.$, Table 4).

Variance was higher in the 400 and 2200 ppm treatments than in the other $\mathrm{CO}_{2}$ treatments for several metrics (total distance, average velocity, turning angle) (Table 5). Overall variability, as demonstrated by the $\mathrm{CV}$ for each metric, was highest in peak velocities, average vertical velocity components, volume transited, and average tortuosity (Table 5). Except for volume transited, as previously noted, and average jet velocity, which had an increasing trend in both variance and $\mathrm{CV}$ with increasing $\mathrm{CO}_{2}\left(\sigma^{2} \mathrm{R}^{2}=0.6506, \mathrm{CV} \mathrm{R}{ }^{2}=0.8647\right)$ variability did not fit an increasing or decreasing trend across $\mathrm{CO}_{2}$ treatments for the $3 \mathrm{D}$ metrics (Table 5). Patterns of variance seen in the 3D metrics do not appear to align with variation in the total number of individuals recorded for each CO2 treatment (Table 1). Although individual paralarvae were quite variable over the course of their swimming path, medians and overall trends of the total dataset were consistent across the entire recorded swimming period (KW, $\mathrm{p}>0.05$ across 10 second time bins within $\mathrm{CO}_{2}$ treatments for all 3D metrics) (Figure 5).

\section{Discussion}

Paralarvae recorded in the 2D behavior arena demonstrated a decrease in time spent in the top, near-surface depth bin at the highest $\mathrm{CO}_{2}$ concentrations tested. Squid paralarvae are negatively buoyant and "hop" with pulsed jetting to maintain their position in between sinks, the rate of which could decrease with decreased available energy (Seibel et al. 2000). Squid jetting comes at a high energetic cost, which has been proposed as a driver of their "live fast, die young" lifestyle and highlighted as a limitation in their ability to compete directly with fish (O'Dor and Webber 1986). Propulsive efficiency, of just the jetting contraction phase, has been shown to be

quite high in $D$. pealeii paralarvae, greater than $80 \%$, decreasing with growth to the juvenile \& 
adult phases (Bartol et al. 2008, 2009b). Modeled hydrodynamic efficiency for squid, considering the whole jetting cycle, however, demonstrated that efficiency increases with growth from hatching to a peak efficiency of about $40 \%$ at $10 \mathrm{~mm}$ DML, decreasing slightly thereafter (Staaf et al. 2014). The decrease in time spent near the surface seen in high $\mathrm{CO}_{2}$-exposed paralarvae in the 2D analysis may have been caused by a reallocation of available energy by these paralarvae towards stress response resulting in decreased swimming activity, a reduction in jetting efficiency due to slightly smaller mantle size (seen in Kaplan et al., 2013), or a combination thereof, resulting in increased time spent sinking. Limitations in the analytical power of the 2D system prevented further disentanglement of these factors.

The significant reductions in average 3D velocity and average positive vertical velocity, as well as the significant decreasing trend in paralarval activity from the 3D system, support the idea that acidification impacts the energetics of the paralarvae, even if effects are slight. Other observed metrics of 3D swimming activity did not demonstrate significant shifts across $\mathrm{CO}_{2}$ levels, but exhibited decreasing patterns with increasing $\mathrm{CO}_{2}$ exposure, further suggesting a subtle impact of acidification on the energetics and dynamics of swimming. Hypercapnia has been shown to depress energy expenditure rates in embryonic and pre-hatchling cuttlefish, Sepia officinalis (Rosa et al. 2013). Adults and juveniles of this species appear capable of withstanding chronic acidification through a system of branchial acid-base transporters, but embryos were seen to a downregulate ion regulatory and metabolic genes under increased acidification (Hu et al. 2011a). Ion pump activities were seen to be even lower in the embryos and paralarvae of a Loliginid squid, Loligo vulgaris, than in S. officinalis, suggesting a greater $\mathrm{pH}$ sensitivity in this taxa (Hu et al. 2010). However, embryos of another squid, Sepioteuthis lessoniana, upregulated genes of a proton secretion pathway under severe chronic acidification $\left(\mathrm{pH}_{\mathrm{nbs}}=7.31\right)$ demonstrating the potential for a powerful homeostatic response (Hu et al. 2013). Given that cephalopod eggs naturally become acidified due to embryonic respiration over the course of development, it is possible that, while variable, a general $\mathrm{pH}$ resilience is a conserved feature of the class, which may serve to explain the weakness in OA responses seen here (Gutowska and Melzner 2009; Long et al. 2016).

The OA effects on vertical swimming in 2013 and decreasing trends in 3D swimming behaviors in 2014 demonstrated a remarkable resilience, requiring intense, chronic exposures (> $1900 \mathrm{ppm}$ ) to acidification that $D$. pealeii should at most experience acutely in estuarine 
environments (Jacobson 2005; Baumann et al. 2015). These levels of acidification are predicted in models only under a high emissions scenario after several hundred years (Caldeira and Wickett 2003). Early physiological work exposing adult $D$. pealeii to hypercapnia noted the remarkable $\mathrm{CO}_{2}$ tolerance of this species, suggesting that even with the Bohr effect, decreased $\mathrm{pH}$ reducing oxygen carrying capacity of their hemocyanin, they would not be significantly stressed unless exposed to concurrent hypoxia (Redfield and Goodkind 1929). Embryos of the California market squid, Doryteuthis opalescens, which naturally experience sporadic $\mathrm{pH}$ reduction (down to 7.65) due to upwelling, showed development delay and smaller statoliths under a combined regime of OA $(\mathrm{pH} 7.57)$ and hypoxia $(80 \mu \mathrm{M})$, while under only decreased $\mathrm{pH}$ (7.56) showed a reduced yolk volume, but larger statoliths compared to hypoxia alone (Navarro et al. 2016). Given the substantial acid-base balancing machinery of cephalopods, it is possible that if subtle effects of acidification are seen, particularly in relation to energy and activity, it is due to a slightly reduced oxygen availability owing to the $\mathrm{pH}$ sensitivity of their hemocyanin, but that they are otherwise fairly resistant to acidification in a well oxygenated system (Hu et al. 2010; Seibel 2013).

While the 2013 2D depth results showed a clear impact of high, chronic acidification on paralarval swimming behavior (Figure 2A), no significant effect of $p \mathrm{CO}_{2}$ on paralarval swimming depth was seen in the 2014 2D trial and all subsequent 3D trials (run in 2014 \& 2015) (Figure 2B, Table 4), indicating a possible interannual or cohort-based variance in the strength of embryonic and paralarval response to this stressor. Seasonal cohorts of L. vulgaris eggs were shown to respond differentially to warming and OA, with the summer clutches showing greater sensitivity (Rosa et al. 2014a). Murray et al. have demonstrated intraspecies seasonal variability in OA stress response for the Atlantic silverside, Menidia menidia, dependent on parental exposure and offspring conditioning (2014). The annual D. pealeii breeding cycle in Vineyard Sound is known to have some weak seasonal structuring with the earliest mating (April-May) involving robust two-year olds, while the rest of the breeding season is dominated by the previous year-class, however our data does not correlate with any clear seasonal signal (Arnold et al. 1974). Squid populations have been noted to demonstrate interannual variability, potentially as a plastic response of the year-class to environmental influence (Pecl et al. 2004a). It is possible that an organism as plastic and fast-lived as D. pealeii could exhibit differential 
responses to stressors dependent on the experiences of the year-class and on how those adults condition their resultant offspring (Summers 1971).

Considerable variability in behavior and response was seen individually within the paralarvae, both overall (Figure 5) and across $\mathrm{CO}_{2}$ treatments (Table 3, Table 5). Seemingly high variance may be partly due to moderate sample sizes in some treatments (Table 1). However, the distributions of variance across $\mathrm{CO}_{2}$ treatments, and the high coefficients of variation for many 3D metrics, indicate a more prominent effect of individual variability (Table 5). Complexity and plasticity are hallmarks of the cephalopods, and individual variability is a strong benefit for fastlived, highly fecund, r-selected organisms such as squid, as it provides adaptive flexibility in the face of rapid environmental change (Herke and Foltz 2002; Pecl et al. 2004a). While variability between cohorts of eggs is likely due to differences in parentage, variability within a clutch is an effect either of differential fathership and/or maternal condition (Buresch et al. 2001, 2009; Steer et al. 2004). Intracapsular variability within a maternal clutch has been noted in the elemental composition of statoliths, DML, and yolk volume in late stage D. opalescens embryos (Navarro et al. 2014, 2016). Given the complexity of squid parentage, differential parental conditioning and any resultant epigenetic effects might also express as differential responses or behaviors, particularly when dealing with a stressor, as is seen in other marine species (Buresch et al. 2001; Miller et al. 2012; Putnam and Gates 2015). Maternal variation in transmitted yolk content could have impacts on both the paralarval energy budget and their specific gravity, both of which could translate to swimming behaviors (Vidal et al. 2002a; Martins et al. 2010a). Embryo position in the egg capsule itself, and the resultant exposure to differential levels of hypoxia and acidification over development, could also explain the dynamic variability in individual swimming behaviors and activities seen in our experiment (Long et al. 2016).

It is likely that our power to see effects caused by OA was reduced by this intense individual variability and the logistical challenge of acquiring a large enough sample size of useable paralarvae videos. Differences in the $2 \mathrm{D}$ system were only seen between those treatment groups with either a substantial sample size of useable videos (400, 1900, and 2200 ppm, Table 1) or a particularly low variance $(1300 \mathrm{ppm}$, Table 3$)$. The videos resulting from the cubic arena provided substantially clearer imagery and allowed both for better observation and tracking of the squid paralarvae (Figure 3, Figure 4). An advantage of the new methodology implemented here is that it is easily set up and repeated. We were limited primarily by our organism's 
breeding window, the low ratio, for our paralarvae, of useable videos to videos taken, and the time needed for experimentation and processing (Table 1)(Vecchione 1981; Hastie et al. 2009).

Three-dimensional positional data acquired through observer corrected automated tracking in Tracker and processing in the 3D model equations described above resulted in clear tracks for each individual, similar to those determined by other established methods (Cachat et al. 2011a). Individual tracked paths produced by the system provide a powerful basis for analyzing movement patterns in marine and aquatic organisms. Total distance traveled and average velocity of the paralarvae were significantly different between the 400 and $1000 \mathrm{ppm}$ groups, but most of the 3D metrics examined demonstrated only weakly decreasing trends with increasing $\mathrm{CO}_{2}$ (Table 4). Average velocities for D. pealeii paralarvae recorded from the 3D system, $8.3 \mathrm{~mm} \mathrm{~s}^{-1} \pm 2.5$ (median $\pm \mathrm{SD}$ ), fall within, but on the low end, of the range previously reported for the species during mantle contraction, $6.6-30.5 \mathrm{~mm} \mathrm{~s}^{-1}$ (Bartol et al. 2009b). Limitations in these metrics may arise from the general, undirected swimming of the organisms. All the animals studied swam at about the same rate and for the same general distance; however, the absence of flow, predators, prey, conspecifics, or other major sensory cues throughout these tests should be noted. It is possible that potential acidification effects in the $3 \mathrm{D}$ swimming system did not emerge, both because of the high multi-scale variability and because the paralarvae were not sufficiently challenged in their motion. Sensory-driven experiments taking advantage of the squid paralarvae's innate photopositivity as a target may better elucidate stress effects by coercing the organism into predictable, directed motion.

Both arenas used likely enacted some influence over the behavior of the paralarvae. Both systems only allowed for a still water experimentation regime and did not provide analysis of the natural impacts of turbulence and flow that other methods, particularly DPIV, are capable of (Fuchs et al. 2004; Wheeler et al. 2015). Stillwater also resulted in a flat surface layer, which was necessary for filming from above, but also allowed for the influence of surface tension, which was found to entrain paralarvae in the meniscus of the $2 \mathrm{D}$ system, and has been shown in other studies to influence the speed and survival of marine larvae (Hidu and Haskin 1978; Yamaoka et al. 2000). Paralarval D. pealeii do not live in a still water environment, but instead navigate the dynamic surface ocean; although their precise behaviors are not well known (Vecchione et al. 2001; Barón 2003; Jacobson 2005). Therefore, while the system was effective at exploring fundamental, physiologically-driven differences in hatchling swimming capability it does not 
directly reflect how paralarval swimming behaviors might shift in response to OA in the natural system or throughout ontogeny.

This study set out to examine the potential impacts of developmental exposure to OA on the swimming behavior of hatchling squid paralarvae. The study required and inspired the development of a novel, simple, and feasible system for recording, tracking, and analyzing the $3 \mathrm{D}$ motion of a motile marine larvae. Early results demonstrated an impact of high $\mathrm{CO}_{2}$ exposures on paralarval activity and vertical positioning. Measured in the 3D system, average velocity and average positive vertical velocity showed significant decreases from ambient, 400 ppm, to $1000 \mathrm{ppm}$, while horizontal velocity showed a significantly decreasing trend further indicating an impact of OA on hatchling energetics. However, most metrics only demonstrated subtle, nonsignificant, decreasing trends with decreased $\mathrm{pH}$, supporting the idea that acidification may be a weak stressor in cephalopods. Notable individual variability, as well as potential interannual and/or cohort scale variability, was also seen in the response to acidification, indicating a substantial plasticity and general $\mathrm{pH}$ resilience for the population. Further study into the physiological tolerances and behavioral responses of this taxon would require incorporating higher $\mathrm{CO}_{2}$ levels, beyond 2200 ppm, or the introduction of compounding stressors in a multifactor design. Replication of this and related stress experiments across multiple years would also be required in order to better describe the patterns and drivers of individual, intra-annual, and interannual variabilities. The 3D model system has potential utility in a wide variety of applications, including complex tracking of aquatic or marine larvae from other taxa, tracking of multiple individuals within the same arena, predator-prey interactions, as well as sensory studies. The range of organismal responses to anthropogenically induced global ocean change continues to grow in diversity and complexity as more taxa and stressors are examined. Improvements in the accessibility of methods to address this ever-expanding field of questions are necessary in order to facilitate and support this substantial effort. 


\section{List of Abbreviations}

2D - Two-dimensional

3D - Three-dimensional

$\mathrm{CV}$ - Coefficient of variation

DML - Dorsal mantle length

DPIV - Digital particle image velocimetry

ESL - Environmental Systems Laboratory

IQR - Interquartile range

KW - Kruskal-Wallis test

MBL - Marine Biological Laboratory

OA - Ocean acidification

SD - Standard deviation

\section{Funding}

This material is based upon work supported by the National Science Foundation Graduate Research Fellowship under Grant No. 1122374. This project is funded by NSF Grant No. 1220034.

\section{Acknowledgments}

We thank D. Remsen, the MBL Marine Resources Center staff, and MBL Gemma crew for their support in acquiring squid. R. Galat and the facilities staff of the WHOI ESL provided system support. D. McCorkle, KYK Chan, and M. White provided valuable insight on the OA system. E. Moberg, A. Beet, and A. Solow assisted in the development and coding of the 3D model system. We also thank E. Bonk, K. Hoering, M. Lee, D. Weiler, and A. Schlunk for their assistance and input with the experiments.

\section{Bioethics}

All applicable international, national, and/or institutional guidelines for the care and use of cephalopods were adhered to. 


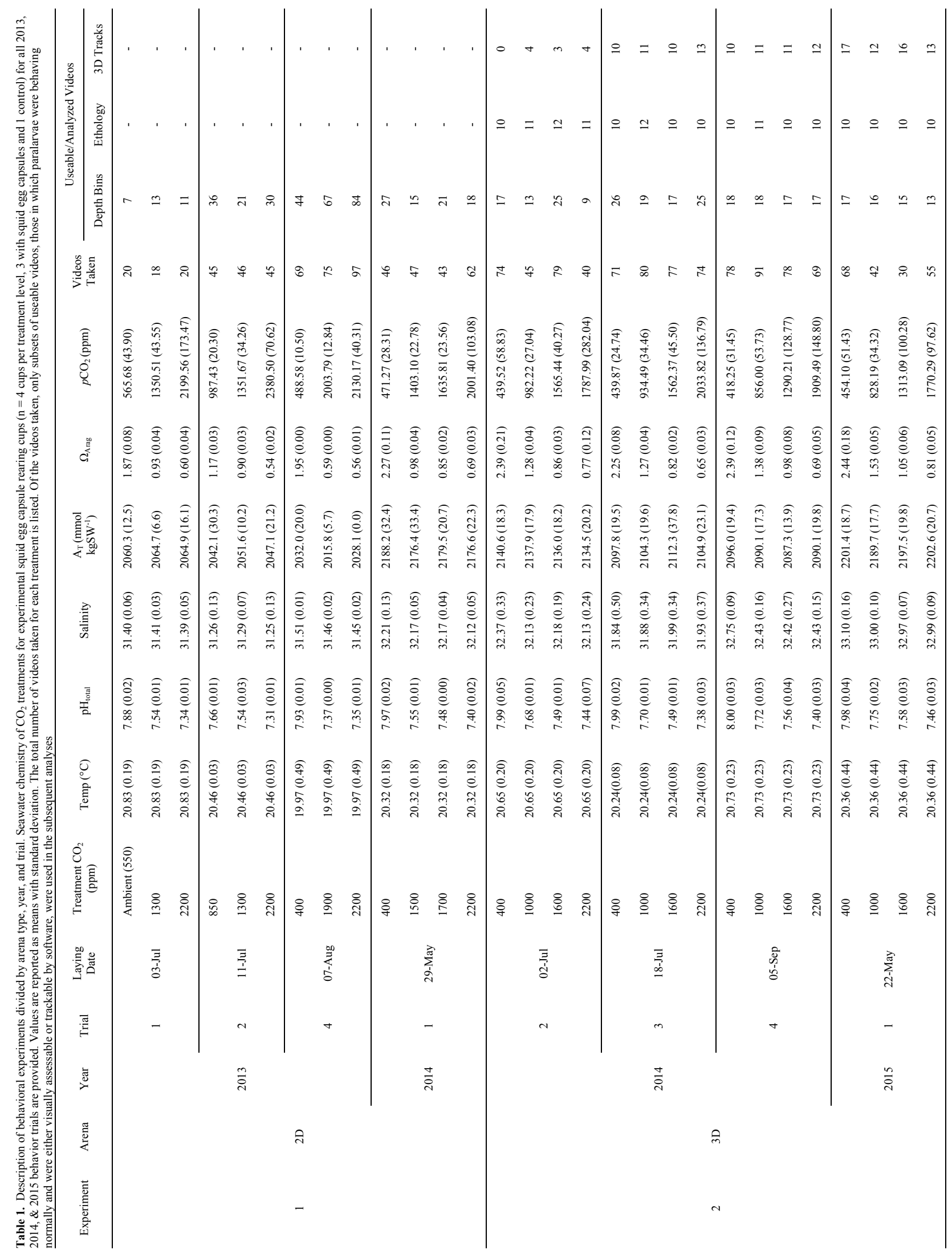


Table 2. Significant Dunn's posthoc test statistics for differences between $\mathrm{CO}_{2}$ treatments within all depth bins examined. Data compiled from all trials of Experiment 1, $\mathrm{Q}_{\text {crit }}=3.12$

\begin{tabular}{lcccccc}
\hline $\mathrm{CO}_{2}$ Treatment $(\mathrm{ppm})$ & 1900 Top & 2200 Top & $1900 \mathrm{Mid}$ & $2200 \mathrm{Mid}$ & 1900 Bottom & 1900 Bottom \\
\hline 400 Top & 3.52 & 3.72 & - & - & - & - \\
1300 Top & 3.97 & 4.06 & - & - & - & - \\
1300 Middle & - & - & 3.21 & 3.36 & - & - \\
1300 Bottom & - & - & - & - & 3.58 & 3.74 \\
\hline
\end{tabular}

Table 3. Variance of $2 \mathrm{D}$ depth bins per $\mathrm{CO}_{2}$ level compiled over all trials of Experiment $1 . \mathrm{R}^{2}$ are of fits of linear trend lines ( $p>0.05$ for all regressions)

\begin{tabular}{lccccccccc}
\hline$\sigma^{2}$ & $\begin{array}{l}400 \\
\mathrm{ppm}\end{array}$ & $\begin{array}{l}550 \\
\mathrm{ppm}\end{array}$ & $\begin{array}{l}850 \\
\mathrm{ppm}\end{array}$ & $\begin{array}{l}1300 \\
\mathrm{ppm}\end{array}$ & $\begin{array}{l}1500 \\
\mathrm{ppm}\end{array}$ & $\begin{array}{l}1700 \\
\mathrm{ppm}\end{array}$ & $\begin{array}{l}1900 \\
\mathrm{ppm}\end{array}$ & $\begin{array}{l}2200 \\
\mathrm{ppm}\end{array}$ & $\mathrm{R}^{2}$ \\
\hline 2D Top & 0.105 & 0.143 & 0.036 & 0.001 & 0.178 & 0.047 & 0.156 & 0.156 & 0.054 \\
2D Middle & 0.006 & 0.000 & 0.007 & 0.001 & 0.027 & 0.033 & 0.015 & 0.036 & 0.609 \\
2D Bottom & 0.091 & 0.129 & 0.023 & 0.000 & 0.117 & 0.005 & 0.156 & 0.116 & 0.015 \\
\hline
\end{tabular}


Table 4. Values of the 3D swimming behavior metrics at each $\mathrm{CO}_{2}$ treatment. Reported values are medians and IQR. Significant Kruskal-Wallis $\mathrm{p}$ values marked with $*(\mathrm{p}<0.05) . \mathrm{R}^{2}$ are of fits of linear trend lines. Significant regressions are marked with $*(p<0.05)$.

\begin{tabular}{|c|c|c|c|c|c|c|}
\hline 3D Metric & $400 \mathrm{ppm}$ & $1000 \mathrm{ppm}$ & $1600 \mathrm{ppm}$ & $2200 \mathrm{ppm}$ & $\begin{array}{l}\text { KW p } \\
\text { value }\end{array}$ & $\mathrm{R}^{2}$ \\
\hline 3D Top (\% Frames) & $\begin{array}{l}0.95 \\
(0.64-1.00)\end{array}$ & $\begin{array}{l}0.88 \\
(0.53-1.00)\end{array}$ & $\begin{array}{l}0.92 \\
(0.72-1.00)\end{array}$ & $\begin{array}{l}0.81 \\
(0.43-0.98)\end{array}$ & 0.1094 & 0.6455 \\
\hline 3D Mid (\% Frames) & $\begin{array}{l}0.01 \\
(0.00-0.12)\end{array}$ & $\begin{array}{l}0.07 \\
(0.00-0.21)\end{array}$ & $\begin{array}{l}0.05 \\
(0.00-0.13)\end{array}$ & $\begin{array}{l}0.06 \\
(0.00-0.17)\end{array}$ & 0.0568 & 0.5030 \\
\hline 3D Bottom (\% Frames) & $\begin{array}{l}0.00 \\
(0.00-0.16)\end{array}$ & $\begin{array}{l}0.03 \\
(0.00-0.17)\end{array}$ & $\begin{array}{l}0.00 \\
(0.00-0.08)\end{array}$ & $\begin{array}{l}0.04 \\
(0.00-0.30)\end{array}$ & 0.0694 & 0.3775 \\
\hline Ethology (\% Active) & $\begin{array}{l}95.8 \\
(88.1-100)\end{array}$ & $\begin{array}{l}93.8 \\
(86.5-100)\end{array}$ & $\begin{array}{l}91.7 \\
(68.5-99.4)\end{array}$ & $\begin{array}{l}88.3 \\
(69.2-100)\end{array}$ & 0.242 & $0.9847^{*}$ \\
\hline Total Distance (mm) & $\begin{array}{l}1128.7 \\
(985.1-1403.9)\end{array}$ & $\begin{array}{l}961.8 \\
(855.7-1116.3)\end{array}$ & $\begin{array}{l}997.9 \\
(912.5-1187.9)\end{array}$ & $\begin{array}{l}985.4 \\
(840.2-1165.6)\end{array}$ & $0.0342 *$ & 0.4590 \\
\hline $\begin{array}{l}\text { Average Velocity } \\
\left(\mathrm{mm} \mathrm{s}^{-1}\right)\end{array}$ & $\begin{array}{l}9.4 \\
(8.2-11.7)\end{array}$ & $\begin{array}{l}8.0 \\
(7.1-9.3)\end{array}$ & $\begin{array}{l}8.3 \\
(7.6-9.9)\end{array}$ & $\begin{array}{l}8.2 \\
(7.0-9.7)\end{array}$ & $0.0354 *$ & 0.4590 \\
\hline Peak Velocity $\left(\mathrm{mm} \mathrm{s}^{-1}\right)$ & $\begin{array}{l}139.6 \\
(99.9-206.9)\end{array}$ & $\begin{array}{l}138.2 \\
(78.8-228.7)\end{array}$ & $\begin{array}{l}108.1 \\
(86.4-163.3)\end{array}$ & $\begin{array}{l}123.9 \\
(82.1-187.5)\end{array}$ & 0.4378 & 0.4578 \\
\hline $\begin{array}{l}\text { Average Jet Velocity } \\
\left(\mathrm{mm} \mathrm{s}^{-1}\right)\end{array}$ & $\begin{array}{l}17.6 \\
(14.2-19.6)\end{array}$ & $\begin{array}{l}14.9 \\
(13.1-16.5)\end{array}$ & $\begin{array}{l}14.7 \\
(13.0-16.9)\end{array}$ & $\begin{array}{l}15.1 \\
(12.5-17.9)\end{array}$ & 0.1192 & 0.5168 \\
\hline Jetting Rate (Jets s ${ }^{-1}$ ) & $\begin{array}{l}2.73 \\
(2.51-3.02)\end{array}$ & $\begin{array}{l}2.70 \\
(2.51-2.89)\end{array}$ & $\begin{array}{l}2.72 \\
(2.47-2.85)\end{array}$ & $\begin{array}{l}2.63 \\
(2.38-2.81)\end{array}$ & 0.3436 & 0.6270 \\
\hline $\begin{array}{l}\text { Average Vertical Velocity } \\
\left(\mathrm{mm} \mathrm{s}^{-1}\right)\end{array}$ & $\begin{array}{l}0.0 \\
(-0.1-0.1)\end{array}$ & $\begin{array}{l}0.0 \\
(-0.1-0.3)\end{array}$ & $\begin{array}{l}0.1 \\
(0.0-0.4)\end{array}$ & $\begin{array}{l}0.0 \\
(-0.1-0.3)\end{array}$ & 0.1935 & 0.0678 \\
\hline $\begin{array}{l}\text { Average Positive Vertical } \\
\text { Velocity }\left(\mathrm{mm} \mathrm{s}^{-1}\right)\end{array}$ & $\begin{array}{l}9.6 \\
(7.9-10.6)\end{array}$ & $\begin{array}{l}8.7 \\
(6.8-9.3)\end{array}$ & $\begin{array}{l}8.7 \\
(7.1-9.6)\end{array}$ & $\begin{array}{l}8.7 \\
(7.4-9.7)\end{array}$ & $0.0126^{*}$ & 0.5540 \\
\hline $\begin{array}{l}\text { Peak Vertical Velocity } \\
\left(\mathrm{mm} \mathrm{s}^{-1}\right)\end{array}$ & $\begin{array}{l}100.3 \\
(75.0-150.6)\end{array}$ & $\begin{array}{l}83.8 \\
(60.6-138.3)\end{array}$ & $\begin{array}{l}85.9 \\
(62.4-127.0)\end{array}$ & $\begin{array}{l}83.2 \\
(66.6-106.9)\end{array}$ & 0.2584 & 0.6194 \\
\hline $\begin{array}{l}\text { Average Negative Vertical } \\
\text { Velocity }\left(\mathrm{mm} \mathrm{s}^{-1}\right)\end{array}$ & $\begin{array}{l}-5.7 \\
(-6.2--4.9)\end{array}$ & $\begin{array}{l}-4.6 \\
(-5.3--3.9)\end{array}$ & $\begin{array}{l}-4.9 \\
(-5.9--4.3)\end{array}$ & $\begin{array}{l}-5.0 \\
(-5.7--4.5)\end{array}$ & $0.0028 *$ & 0.2847 \\
\hline $\begin{array}{l}\text { Minimum Vertical Velocity } \\
\left(\mathrm{mm} \mathrm{s}^{-1}\right)\end{array}$ & $\begin{array}{l}-83.5 \\
(-122.2--62.8)\end{array}$ & $\begin{array}{l}-63.1 \\
(-118.6--44.5)\end{array}$ & $\begin{array}{l}-74.9 \\
(-101.4--54.6)\end{array}$ & $\begin{array}{l}-79.9 \\
(-117.1--49.5)\end{array}$ & 0.4982 & 0.0002 \\
\hline $\begin{array}{l}\text { Average Horizontal } \\
\text { Velocity }\left(\mathrm{mm} \mathrm{s}^{-1}\right)\end{array}$ & $\begin{array}{l}4.6 \\
(3.1-6.2)\end{array}$ & $\begin{array}{l}4.3 \\
(3.2-5.1)\end{array}$ & $\begin{array}{l}3.7 \\
(3.0-5.1)\end{array}$ & $\begin{array}{l}3.4 \\
(2.8-4.6)\end{array}$ & 0.1945 & $0.9798 *$ \\
\hline $\begin{array}{l}\text { Peak Horizontal Velocity } \\
\left(\mathrm{mm} \mathrm{s}^{-1}\right)\end{array}$ & $\begin{array}{l}108.8 \\
(72.7-138.8)\end{array}$ & $\begin{array}{l}112.6 \\
(61.2-174.5)\end{array}$ & $\begin{array}{l}86.8 \\
(58.6-107.5)\end{array}$ & $\begin{array}{l}78.7 \\
(67.8-149.1)\end{array}$ & 0.2712 & 0.8224 \\
\hline Volume Transited $\left(\mathrm{mm}^{3}\right)$ & $\begin{array}{l}53,786 \\
(20,550- \\
95,664)\end{array}$ & $\begin{array}{l}65,076 \\
(33,646- \\
125,970)\end{array}$ & $\begin{array}{l}53,314 \\
(30,734- \\
103,950)\end{array}$ & $\begin{array}{l}46,406 \\
(22,078- \\
118,882)\end{array}$ & 0.7416 & 0.3207 \\
\hline $\begin{array}{l}\text { Average Turn Angle } \\
\text { (degrees) }\end{array}$ & $\begin{array}{l}56.53 \\
(48.84-64.54)\end{array}$ & $\begin{array}{l}53.49 \\
(48.45-62.04)\end{array}$ & $\begin{array}{l}52.05 \\
(45.92-60.00)\end{array}$ & $\begin{array}{l}52.30 \\
(43.64-63.71)\end{array}$ & 0.4334 & 0.7863 \\
\hline Average Tortuosity & $\begin{array}{l}3.43 \\
(3.00-4.79)\end{array}$ & $\begin{array}{l}3.46 \\
(2.97-4.35)\end{array}$ & $\begin{array}{l}3.39 \\
(2.92-4.51)\end{array}$ & $\begin{array}{l}3.63 \\
(3.07-5.19)\end{array}$ & 0.6730 & 0.4065 \\
\hline
\end{tabular}


Table 5. Variance and coefficient of variation (CV) both per $\mathrm{CO}_{2}$ treatment and overall for all of the $3 \mathrm{D}$ arena metrics. $\mathrm{R}^{2}$ are of fits of linear trend lines ( $>0.05$ for all regressions)

\begin{tabular}{|c|c|c|c|c|c|c|c|}
\hline 3D Metric & & $400 \mathrm{ppm}$ & $1000 \mathrm{ppm}$ & $1600 \mathrm{ppm}$ & $2200 \mathrm{ppm}$ & $\mathrm{R}^{2}$ & Overall \\
\hline \multirow{2}{*}{ 3D Top (\% Frames) } & $\sigma^{2}$ & 0.1251 & 0.1007 & 0.0793 & 0.1162 & 0.0956 & 0.1073 \\
\hline & $\mathrm{CV}$ & 0.473 & 0.440 & 0.352 & 0.503 & 0.0000 & 0.443 \\
\hline \multirow{2}{*}{ 3D Mid (\% Frames) } & $\sigma^{2}$ & 0.0114 & 0.0210 & 0.0106 & 0.0131 & 0.0196 & 0.0143 \\
\hline & $\mathrm{CV}$ & 1.48 & 1.16 & 1.22 & 1.09 & 0.7038 & 1.26 \\
\hline \multirow{2}{*}{ 3D Bottom (\% Frames) } & $\sigma^{2}$ & 0.1107 & 0.0592 & 0.0585 & 0.1059 & 0.0048 & 0.0852 \\
\hline & $\mathrm{CV}$ & 1.85 & 1.59 & 2.10 & 1.50 & 0.0637 & 1.77 \\
\hline \multirow{2}{*}{ Ethology (\% Active) } & $\sigma^{2}$ & 593 & 597 & 910 & 590 & 0.0607 & 685 \\
\hline & $\mathrm{CV}$ & 0.284 & 0.291 & 0.393 & 0.303 & 0.1615 & 0.321 \\
\hline \multirow{2}{*}{ Total Distance (mm) } & $\sigma^{2}$ & 99,095 & 58,994 & 65,760 & 106,467 & 0.0248 & 87,981 \\
\hline & $\mathrm{CV}$ & 0.265 & 0.241 & 0.240 & 0.314 & 0.2961 & 0.275 \\
\hline \multirow{2}{*}{ Average Velocity $\left(\mathrm{mm} \mathrm{s}^{-1}\right)$} & $\sigma^{2}$ & 6.81 & 4.10 & 4.56 & 7.39 & 0.0305 & 6.08 \\
\hline & $\mathrm{CV}$ & 0.264 & 0.241 & 0.240 & 0.314 & 0.3072 & 0.275 \\
\hline \multirow{2}{*}{ Peak Velocity $\left(\mathrm{mm} \mathrm{s}^{-1}\right)$} & $\sigma^{2}$ & 95,801 & 192,554 & 51,533 & 341,842 & 0.3596 & 177,644 \\
\hline & $\mathrm{CV}$ & 1.20 & 1.56 & 1.33 & 1.89 & 0.3072 & 1.64 \\
\hline \multirow{2}{*}{ Average Jet Velocity $\left(\mathrm{mm} \mathrm{s}^{-1}\right)$} & $\sigma^{2}$ & 14.74 & 11.34 & 16.80 & 25.37 & 0.6506 & 17.99 \\
\hline & $\mathrm{CV}$ & 0.221 & 0.221 & 0.258 & 0.314 & 0.8647 & 0.262 \\
\hline \multirow{2}{*}{ Jetting Rate (Jets s $\left.{ }^{-1}\right)$} & $\sigma^{2}$ & 0.1553 & 0.1556 & 0.0806 & 0.2751 & 0.2083 & 0.1745 \\
\hline & $\mathrm{CV}$ & 0.145 & 0.150 & 0.106 & 0.208 & 0.1974 & 0.158 \\
\hline \multirow{2}{*}{$\begin{array}{l}\text { Average Vertical Velocity } \\
\left(\mathrm{mm} \mathrm{s}^{-1}\right)\end{array}$} & $\sigma^{2}$ & 0.067 & 0.158 & 0.102 & 0.126 & 0.1664 & 0.116 \\
\hline & $\mathrm{CV}$ & 9.63 & 6.80 & 2.24 & 5.65 & 0.4854 & 4.61 \\
\hline \multirow{2}{*}{$\begin{array}{l}\text { Average Positive Vertical } \\
\text { Velocity }\left(\mathrm{mm} \mathrm{s}^{-1}\right)\end{array}$} & $\sigma^{2}$ & 4.87 & 5.19 & 3.15 & 4.75 & 0.1147 & 4.89 \\
\hline & $\mathrm{CV}$ & 0.232 & 0.293 & 0.207 & 0.260 & 0.0001 & 0.258 \\
\hline \multirow{2}{*}{$\begin{array}{l}\text { Peak Vertical Velocity } \\
\left(\mathrm{mm} \mathrm{s}^{-1}\right)\end{array}$} & $\sigma^{2}$ & 13,692 & 45,967 & 4,545 & 4,056 & 0.2109 & 17,112 \\
\hline & $\mathrm{CV}$ & 0.823 & 1.48 & 0.645 & 0.626 & 0.2097 & 1.07 \\
\hline \multirow{2}{*}{$\begin{array}{l}\text { Average Negative Vertical } \\
\text { Velocity }\left(\mathrm{mm} \mathrm{s}^{-1}\right)\end{array}$} & $\sigma^{2}$ & 2.02 & 1.65 & 1.50 & 2.22 & 0.0338 & 2.02 \\
\hline & $\mathrm{CV}$ & 0.248 & 0.280 & 0.235 & 0.288 & 0.1519 & 0.274 \\
\hline \multirow{2}{*}{$\begin{array}{l}\text { Minimum Vertical Velocity } \\
\left(\mathrm{mm} \mathrm{s}^{-1}\right)\end{array}$} & $\sigma^{2}$ & 14,914 & 48,003 & 4,191 & 179,322 & 0.5174 & 65,185 \\
\hline & $\mathrm{CV}$ & 0.988 & 1.73 & 0.718 & 2.68 & 0.3575 & 2.04 \\
\hline \multirow{2}{*}{$\begin{array}{l}\text { Average Horizontal Velocity } \\
\left(\mathrm{mm} \mathrm{s}^{-1}\right)\end{array}$} & $\sigma^{2}$ & 4.30 & 2.86 & 3.29 & 4.57 & 0.0389 & 3.86 \\
\hline & $\mathrm{CV}$ & 0.429 & 0.378 & 0.414 & 0.523 & 0.4426 & 0.442 \\
\hline \multirow{2}{*}{$\begin{array}{l}\text { Peak Horizontal Velocity } \\
\left(\mathrm{mm} \mathrm{s}^{-1}\right)\end{array}$} & $\sigma^{2}$ & 94,830 & 158,355 & 49,637 & 185,639 & 0.1181 & 125,379 \\
\hline & $\mathrm{CV}$ & 1.44 & 1.72 & 1.68 & 1.91 & 0.8284 & 1.76 \\
\hline \multirow{2}{*}{ Volume Transited $\left(\mathrm{mm}^{3}\right)$} & $\sigma^{2}$ & $2.86 \times 10^{9}$ & $4.39 \times 10^{9}$ & $4.56 \times 10^{9}$ & $7.71 \times 10^{9}$ & 0.8710 & $5.06 \times 10^{9}$ \\
\hline & $\mathrm{CV}$ & 0.820 & 0.785 & 0.919 & 1.04 & 0.8015 & 0.918 \\
\hline \multirow{2}{*}{ Average Turn Angle (degrees) } & $\sigma^{2}$ & 238.7 & 181.8 & 158.3 & 241.7 & 0.0020 & 211.0 \\
\hline & $\mathrm{CV}$ & 0.262 & 0.248 & 0.236 & 0.281 & 0.0890 & 0.261 \\
\hline \multirow{2}{*}{ Average Tortuosity } & $\sigma^{2}$ & 33.51 & 3.18 & 3.59 & 37.56 & 0.0076 & 20.20 \\
\hline & $\mathrm{CV}$ & 1.18 & 0.430 & 0.478 & 1.09 & 0.0054 & 0.959 \\
\hline
\end{tabular}


Table 6. Significant Dunn's posthoc test statistics for differences between $\mathrm{CO}_{2}$ treatments in 3D metrics, $\mathrm{Q}_{\text {crit }}=2.631$

\begin{tabular}{lcccc}
\hline Metric: Treatment & $400 \mathrm{ppm}$ & $1000 \mathrm{ppm}$ & $1600 \mathrm{ppm}$ & $2200 \mathrm{ppm}$ \\
\hline Total Distance $(\mathrm{mm}): 400 \mathrm{ppm}$ & - & 2.710 & - & - \\
Average Velocity $\left(\mathrm{mm} \mathrm{s}^{-1}\right): 400 \mathrm{ppm}$ & - & 2.700 & - & - \\
Average Positive Vertical Velocity $\left(\mathrm{mm} \mathrm{s}^{-1}\right): 400 \mathrm{ppm}$ & - & 3.200 & - & - \\
Average Negative Vertical Velocity $\left(\mathrm{mm} \mathrm{s}^{-1}\right): 400 \mathrm{ppm}$ & - & 3.741 & - & - \\
\hline
\end{tabular}


A

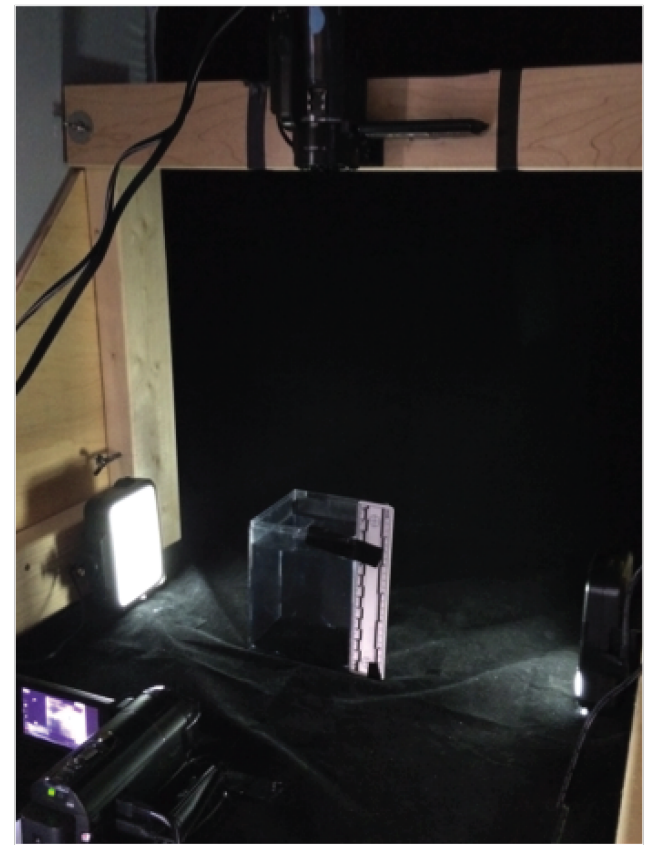

$\mathrm{C}$

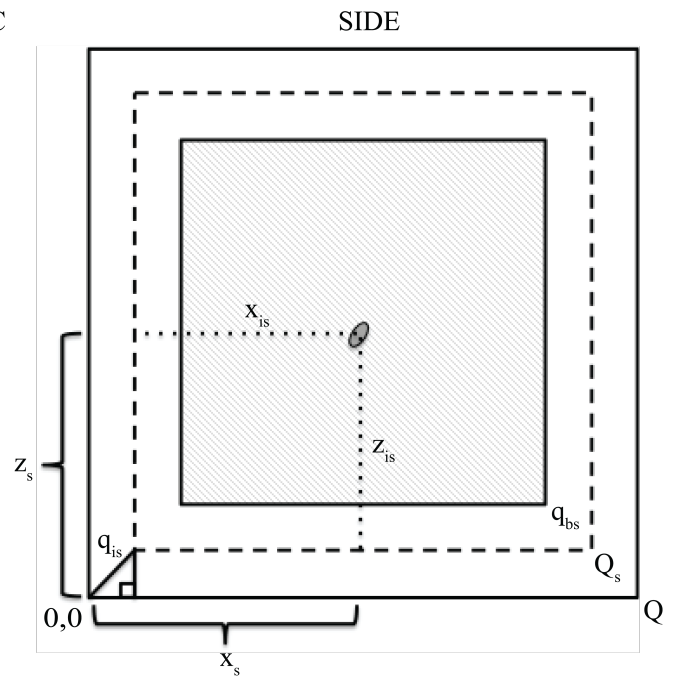

B
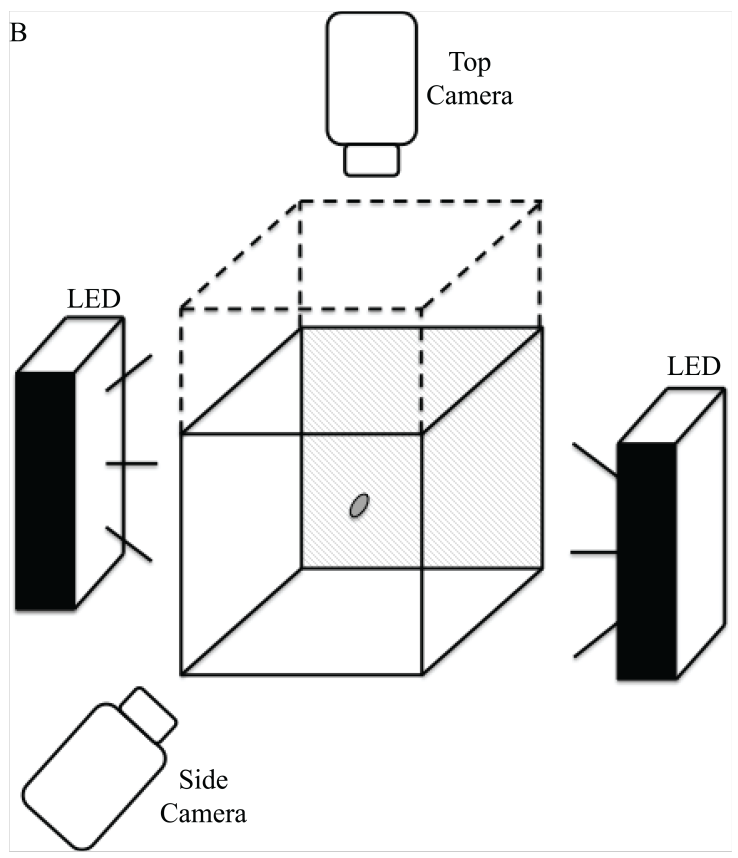

$\mathrm{D}$

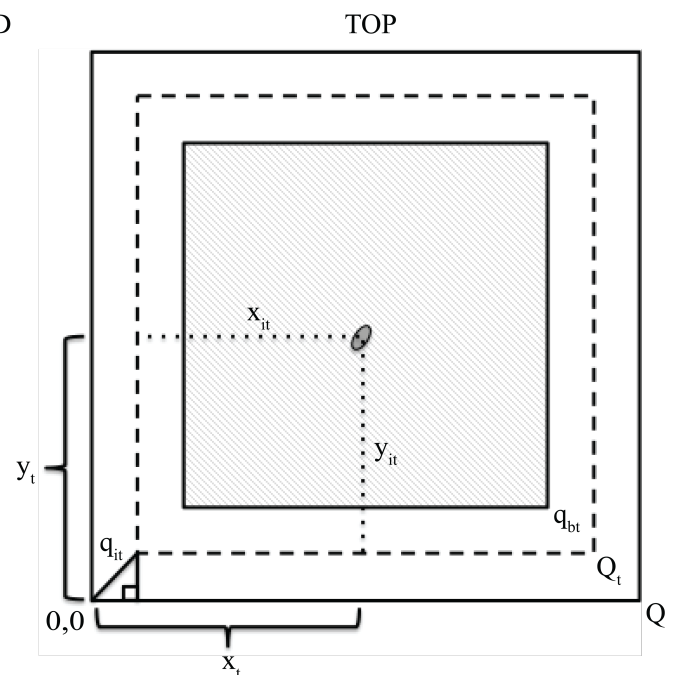

Figure 1. (A) The set-up for 3D behavior recording placed inside of the tarp-covered photobox showing the wooden frame used to mount the top-facing video camera, the 3D arena in center, with ruler attached for scale, flanked by LED panels on each side, and the front facing video camera. (B) A schematic of the arena set-up showing relative placement of the arena, cameras, and lights. Solid lines indicate seawater volume, while dotted lines indicate arena volume (not to scale, see supplementary Fig. S2). The accompanying model system for the side view (C) and top view (D) to correct positional data for the effect of diminishing axes frames 
Figure 2. The proportion of paralarval time spent in the top depth bin in the $2 \mathrm{D}$ arena of Experiment 1 across $\mathrm{CO}_{2}$ treatments from trials in 2013 (A), 2014 (B), and all trials compiled (C). Dotted circles denote medians and plus signs denote outliers. Lower case letters denote statistical groups 

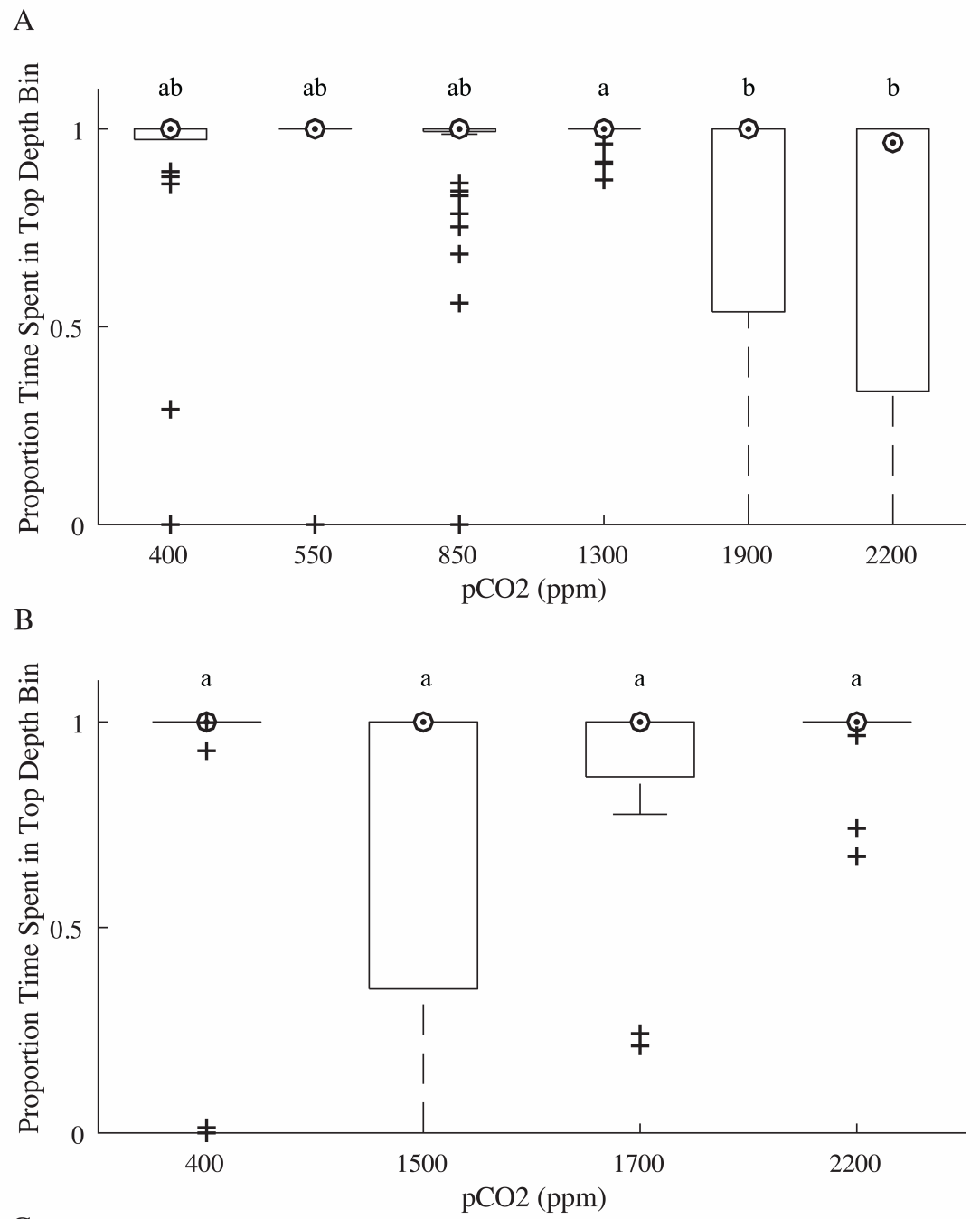

C

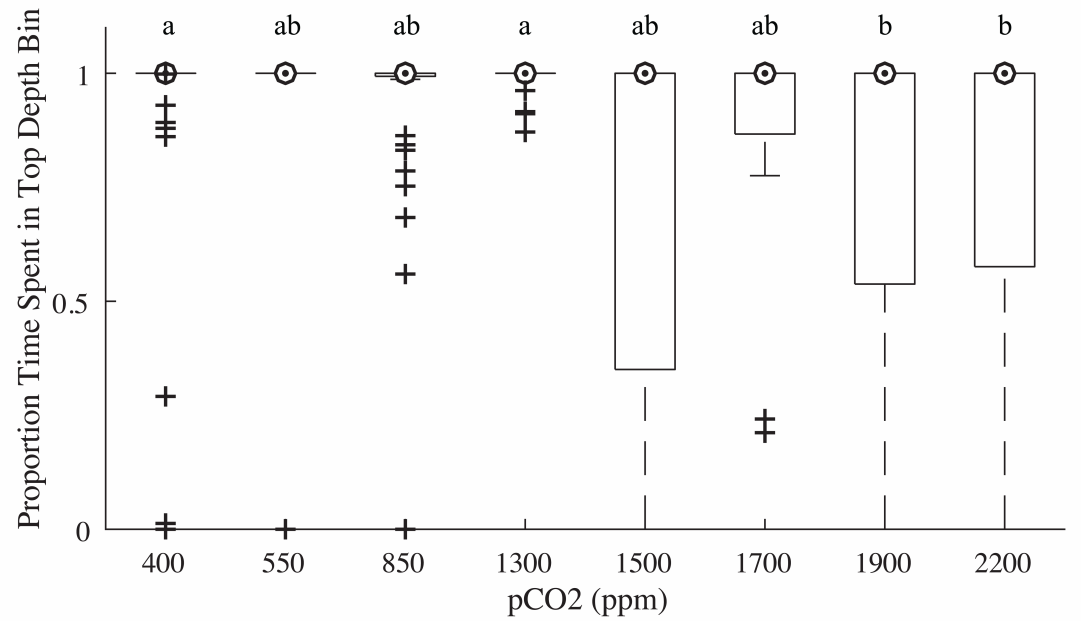


Figure 3. (A) Vertical swimming profile for an ambient (400 ppm) D. pealeii paralarvae (individual 69_69_08_02) showing depth (blue), vertical velocity (red), and vertical acceleration (gray) over the entire 120 -second recording period. (B) A ten-second slice of the swimming profile in A, from 20 seconds to 30 seconds in the video, shows the paralarvae made "hop and sink" jets during descent, rapid ascent, and slow ascent. The velocity peaks represent individual vertical jets 

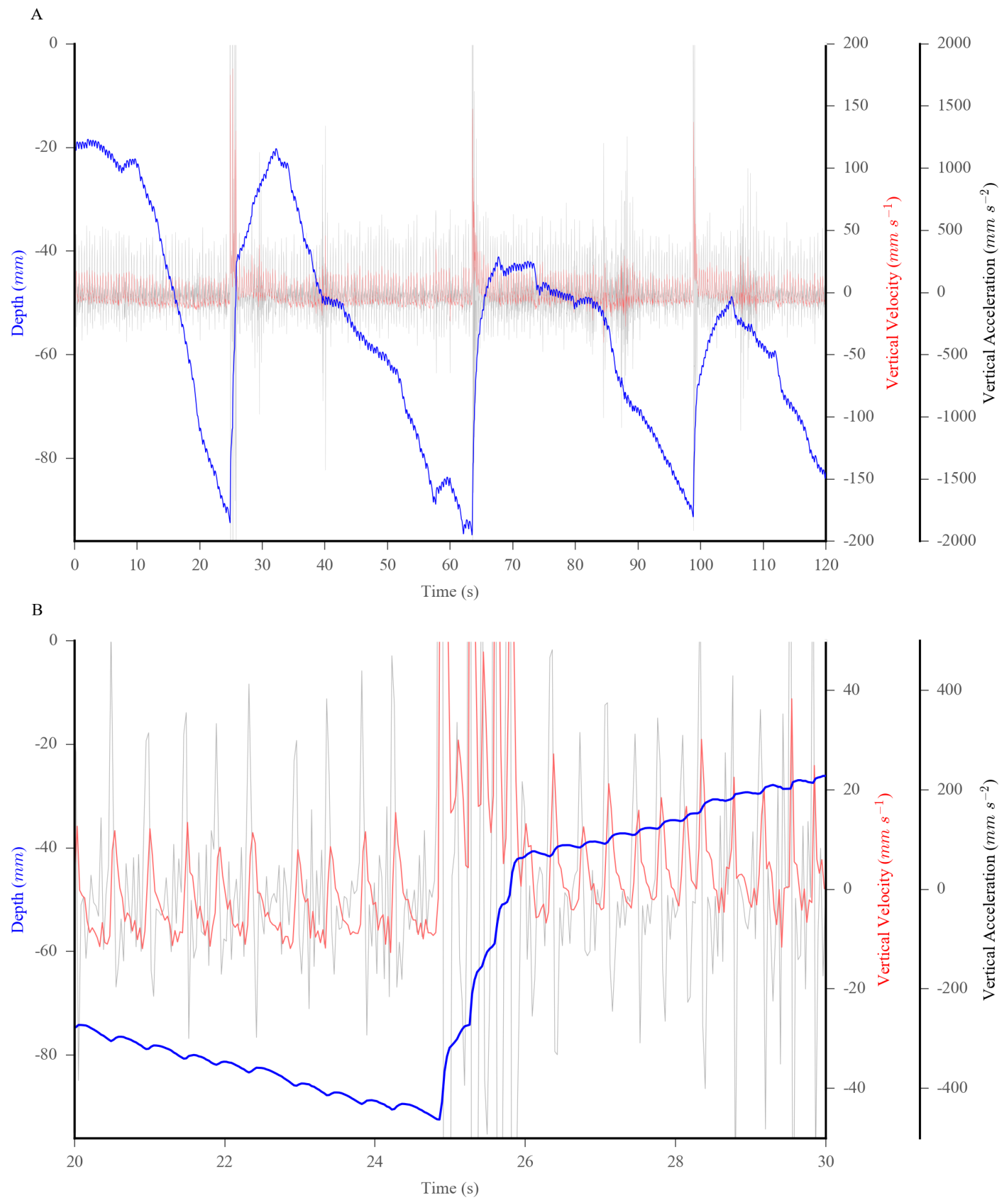
A
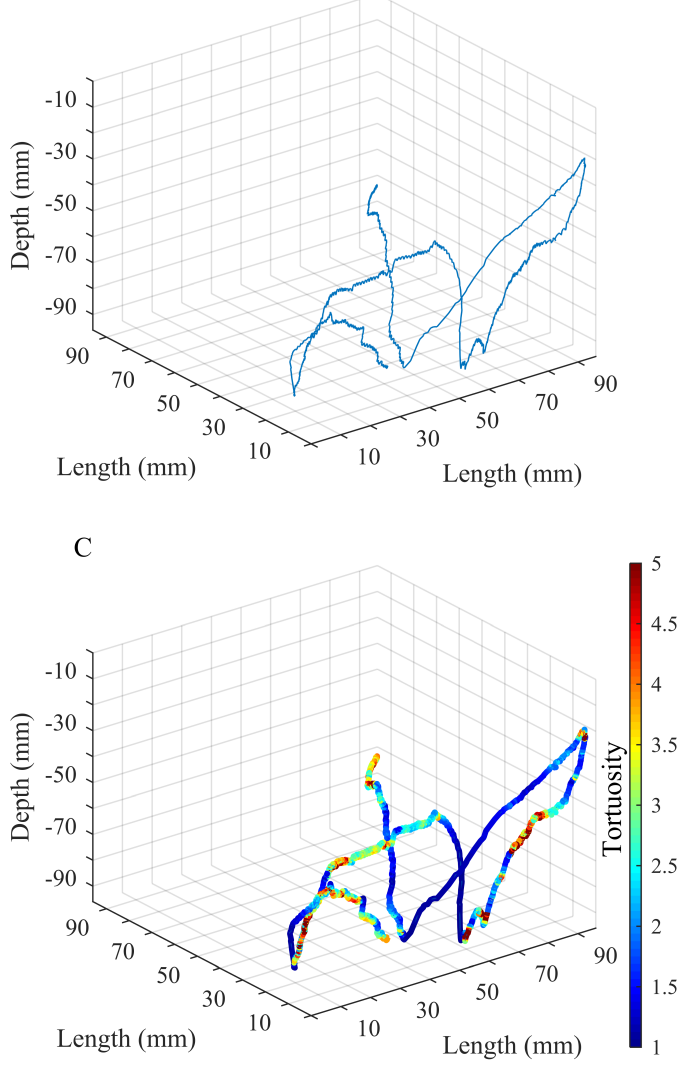

B
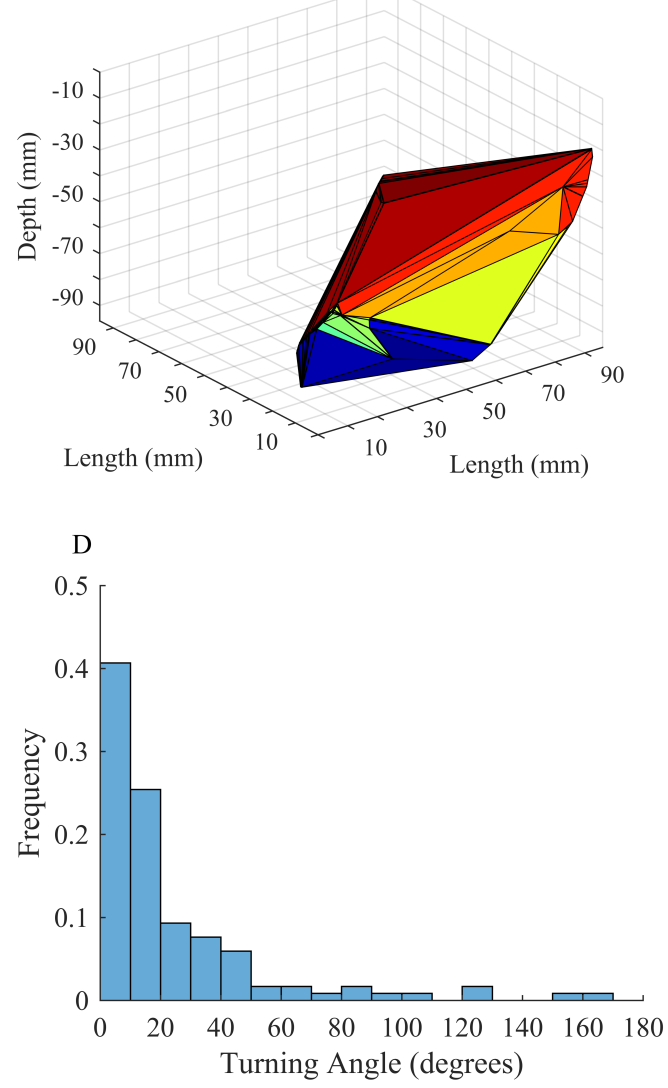

Figure 4. (A) Three-dimensional track of a swimming path for an ambient (400 ppm) D. pealeii paralarvae (individual 69_69_08_02). The front of the arena, which the side camera was pointed at, was the right axis face. (B) Three-dimensional polygon of volume transited by the paralarvae during recording. (C) Tortuosity of path traversed by the paralarvae sampled on a sliding frame of one-second path segments. (D) Turning angles along paralarval path, sampled at sequential vectors of one-second path segments 
Figure 5. (A) Three-dimensional velocity averaged in ten-second bins across the 120 -second recording period. Each line represents an individual paralarvae, with line color denoting their $\mathrm{CO}_{2}$ treatment (400 ppm, blue; 1000 ppm, green; 1600 ppm, orange; 2200 ppm, red). The thick lines represent the median values for all individuals compiled per each $\mathrm{CO}_{2}$ treatment. Although individuals are remarkably variable over their path and overall, the median lines demonstrate both the decrease in velocity at exposures above $400 \mathrm{ppm}$ and the consistency in overall behavior over time in the arena. (B) The time-binned average velocity data (lines denote medians, colors denote $\mathrm{CO}_{2}$ treatment as in $\mathrm{A}$ ) reinforces the high variability in the whisker length and number of outliers (represented as plus signs), and shows both consistently higher velocities in the $400 \mathrm{ppm}$ treatment and broadly consistent median values within $\mathrm{CO}_{2}$ treatments across the recording time 


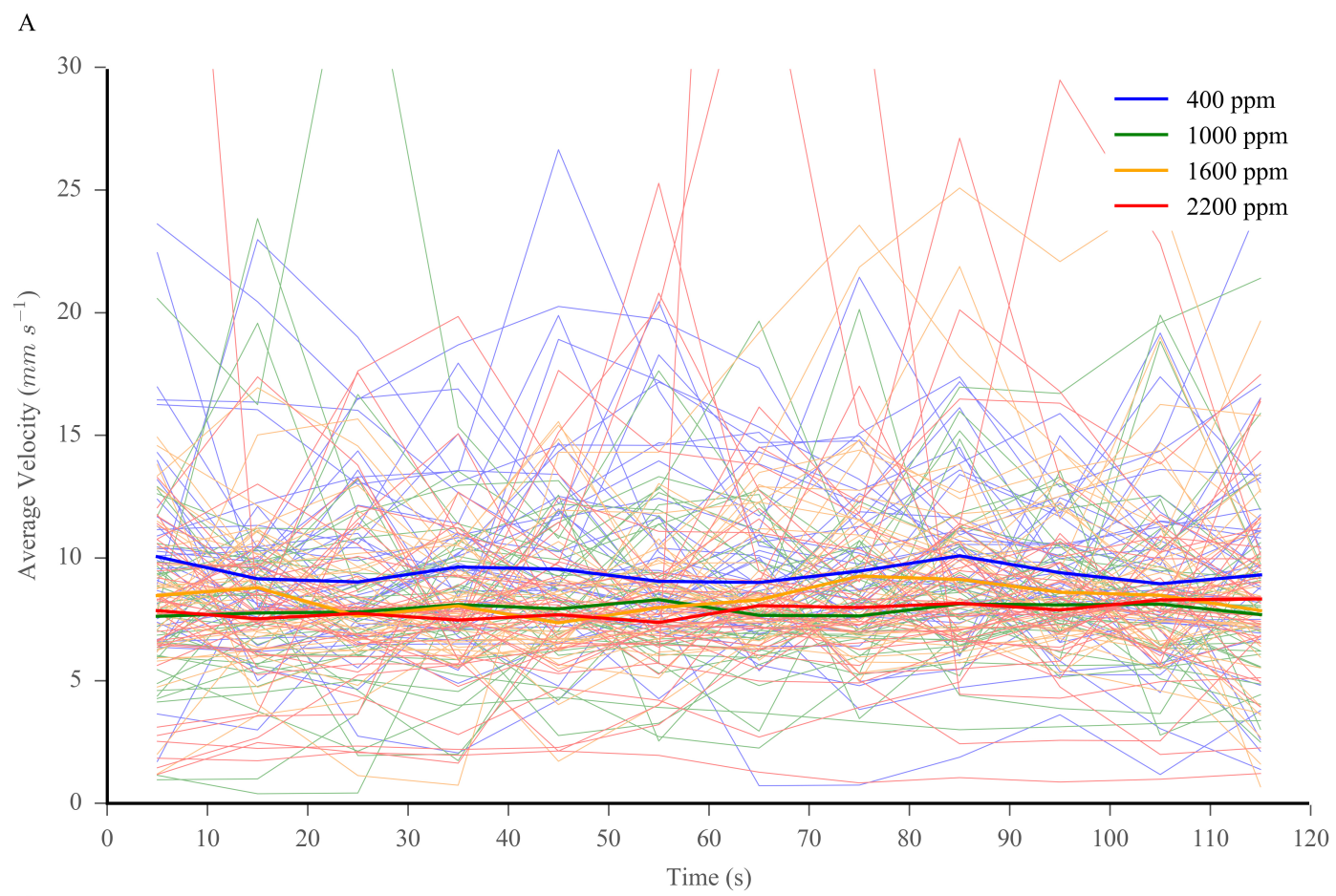

B

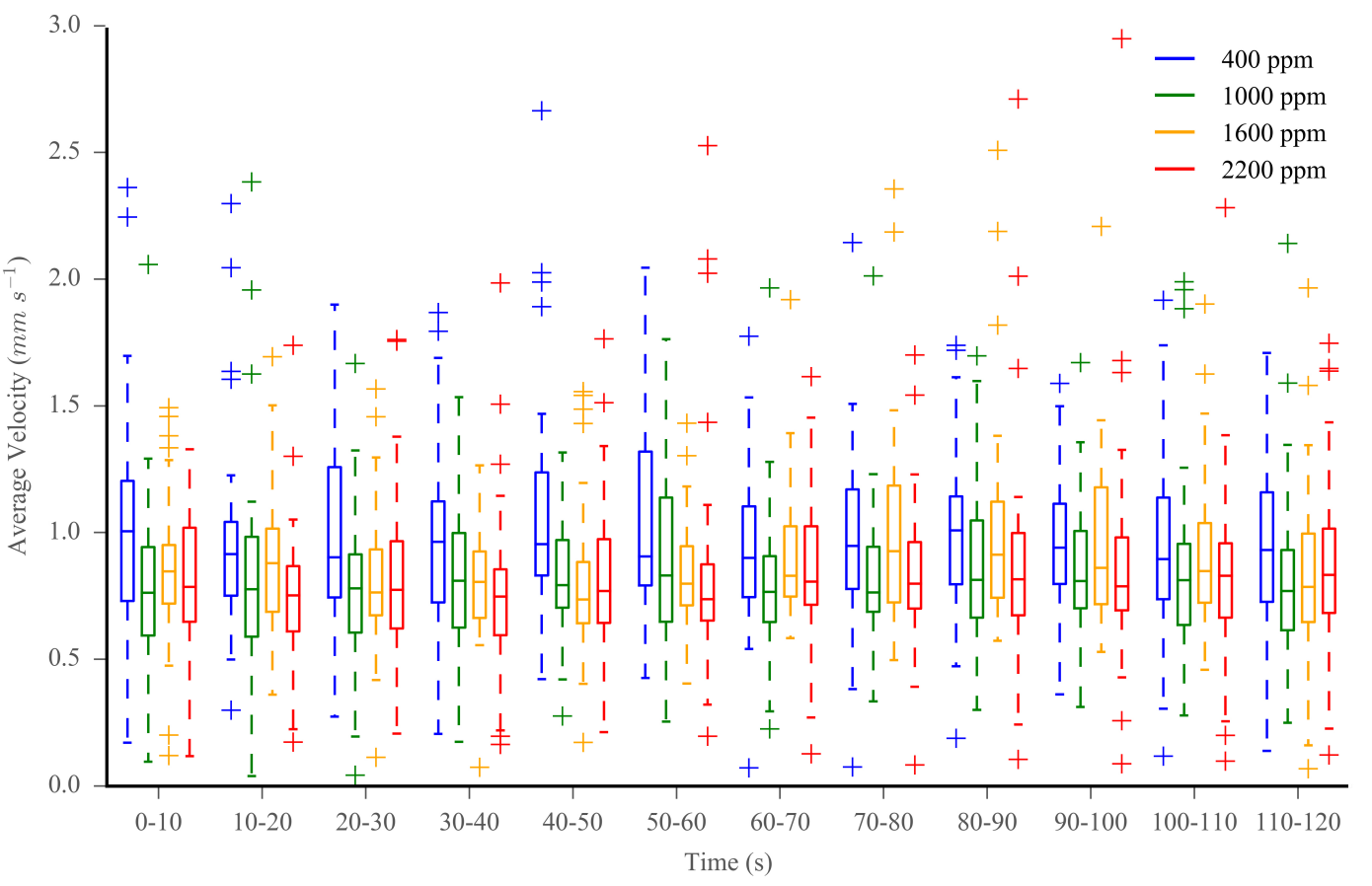




\section{Chapter 4}

Interannual and seasonal variability in the response of squid embryos and paralarvae to ocean acidification

In preparation for submission to Global Change Biology. The Supplementary Materials for this chapter can be found in Appendix C. 


\begin{abstract}
Ocean acidification is occurring rapidly, particularly in coastal systems where buffering capacity is reduced by riverine input and human impacts. Across taxa, developing embryos raised under high exposures of acidification have shown impacts to their development, physiology, and behavior. Most studies have been run with discrete replicates within a single breeding year; however cohorts may vary substantially from year-to-year, with OA responses subsequently varying, reflecting a taxon's resiliency. In the northwest Atlantic, the longfin squid, Doryteuthis pealeii, comes inshore to breed, leaving thousands of encapsulated embryos tied to the nearshore benthos. Seasonal variability in acidification response has been observed in this species, but little has been done to explore this potential plasticity more robustly. Here, we compile data from five years of experiments $(2011,2013$ - 2016), in which we reared squid under a range of acidification exposures (400 - 2200 ppm $\mathrm{CO}_{2}$ ) and examined embryonic development (hatching time and hatching success) and morphology and physiology (dorsal mantle length and yolk sac volume) of the paralarvae. Effect sizes, characterized via response ratios, are used to examine trends within breeding seasons and across years. Response intensity shifted across trials within sensitive years $(2011,2013$, and 2016), suggesting higher variability at the tails of the breeding season. Baseline paralarval state consistently shifted across the season, which strongly correlated with ambient temperature, suggesting conditioning from the parental environment. Further, sensitivity to acidification varied on an interannual scale, likely an impact of the environmental history of the spawning cohorts. These data illustrate the adaptability and sensitivity of a shortlived, highly fecund, and plastic organism, allowing for new insights into understanding how such taxa may respond to climate stressors. Additionally, this work emphasizes the need to run long-term studies to understand population variability as part of assessing potential winners and losers under global ocean change.
\end{abstract}




\section{Introduction}

Ocean acidification (OA) is a process of anthropogenic change occurring, and affecting marine ecosystems, across spatial and temporal and biological scales (Sabine et al. 2004; Fabry et al. 2008; Doney et al. 2009; Pachauri and Meyer 2014). OA is also a variable stressor, not occurring at the same rate in all regions, which interacts with other variable stressors such as warming to produce a complex system of global change that is difficult to recreate in the laboratory (Hobday and Pecl 2014; Gledhill et al. 2015; Kroeker et al. 2017). Research into the impacts of OA on marine organisms has been comprised largely of small scale, short-term exposure experiments, which provide vital information about the physiological capacities of the study organism, but little mechanistic understanding of OA impacts or context for the variability of the stress response (Browman 2016). Long-term organismal studies are challenging both to fund and to perform, but are critical for a greater understanding of an organism's, and its population's, potential for resistance, plasticity, and adaptation under anthropogenic stress (Baumann et al. 2018). Observations of response consistency and variability come through experimental designs that consider the life history and potential sources for variability in the organism and through robust replication. This is even more important if the system the organism comes from is itself substantially variable.

Coastal systems are changing much more rapidly and variably under anthropogenic drivers than the open ocean, with levels of acidification and warming occurring now, on short time scales, which won't be seen for decades to centuries in the wider ocean (Caldeira and Wickett 2003; Feely et al. 2010; Baumann et al. 2015; Gledhill et al. 2015). Concurrent with the regional and temporal variability of coastal systems, is a complex of biology across life history stages. Nearshore environments serve as nursery habitats for a range of marine taxa, which often includes seasonal migrations of these organisms from the shelf and open ocean (Auster and Shackell 2000; Beck et al. 2001; Jacobson 2005). These seasonal breeding aggregations provide the foundation for the next generation, and their success, as well as the success of their offspring, determines the sustainability of the population (Pecl and Moltschaniwskyj 2006;

Moltschaniwskyj and Pecl 2007; Murray et al. 2014). Within a breeding season, environmental influence has been shown to precondition parents, resulting in differential sensitivity of the offspring to stressors such as acidification (Murray et al. 2014; Putnam and Gates 2015; Schunter et al. 2016). Conditions in these systems can vary from year to year as well, and thus so can the 
experience and condition of the potential parents, resulting in annual scale, year-class variability in these populations (Brodziak and Hendrickson 1999; Pecl et al. 2004a; Rodhouse et al. 2014). These epigenetic, transgenerational effects are only beginning to be observed and understood in those marine species that are more visibly threatened by OA, such as corals, but similar processes may influence sensitivity in many taxa (Ross et al. 2012; Jensen et al. 2014; Munday 2014; Schunter et al. 2018). Describing and attempting to understand this variability in response to global processes of ocean change, in part by concurrently linking environmental observations to laboratory studies, is key to building better models and making predictions of species success, ecological interactions, and ecosystem management (Kroeker et al. 2013, 2014).

Myopsid squids, the coastal squids, are a core component of nearshore food webs and are an important human protein source, comprising valuable fisheries (Jacobson 2005; Rosa et al. 2014b; NOAA 2019). Cephalopods are a unique taxon among the marine invertebrates. They are primarily short-lived (1-2 year), mostly highly fecund, and notorious for flexibility, and so are thought to be well poised to maintain viable populations under global ocean change (Doubleday et al. 2016). Studies of cephalopod response to OA have been primarily short-term, showing a range of responses from hypercalcification of the cuttlebone in cuttlefish to changes in activity and behavior in pygmy squid (Gutowska et al. 2008; Spady et al. 2014). Physiologically, squid have been of particular interest because they are thought to exist constantly near the limits of their aerobic scope (Pörtner 1990, 1994; Seibel 2016). However, recent research has demonstrated that adult squids are able to maintain oxygenation of tissue and do not show metabolic suppression under acidification (Birk et al. 2018; Spady et al. 2019).

The response of squid embryos to acidification and related stressors has been another active avenue of research, focusing on developmental impacts and the state of the hatchling paralarvae (Kaplan et al. 2013; Navarro et al. 2014, 2016; Rosa et al. 2014a; Zakroff et al. 2018, 2019). Early life stages of marine organisms are often thought to be more sensitive to environmental stress than adults due to a lack of existing homeostatic machinery (Byrne 2011; Haigh et al. 2015). There are suggestions however, that developing embryos, particularly encapsulated ones, may be built to handle natural variability (Hamdoun and Epel 2007; Noisette et al. 2014). Whether these systems are capable of managing under anthropogenically-boosted stressor variability is less certain. Embryos, removed from the capsule, of the European market squid, Loligo vulgaris, demonstrated metabolic suppression, developmental delay, and increased 
premature hatching under decreased $\mathrm{pH}$, suggesting potential sensitivity for this life stage (Rosa et al. 2014a). Developing embryos of the big fin squid, Sepioteuthis lessoniana, however, have been shown to increase expression of epithelial ion transporters under increased acidification, suggesting substantive mechanisms for resistance to acidification (Hu et al. 2013, 2014).

The inshore longfin squid, Doryteuthis pealeii, comes to the nearshore of the northwest Atlantic coast to breed, entering Vineyard Sound, MA from May - October every year (Arnold et al. 1974; Mesnil 1977; Jacobson 2005). Limits of egg laying habitat for this squid have been described as coastal soft bottom areas with temperatures ranging from $10-23^{\circ} \mathrm{C}$, salinities of 30 - $32 \mathrm{ppt}$, and an expected $\mathrm{pH}_{\mathrm{t}}$ range of 8.2 - 7.88 (250 - $600 \mathrm{ppm} \mathrm{CO}_{2}$ ), but the specific variability these eggs experience has not been directly observed to our knowledge (Jacobson 2005; Zakroff et al. 2019). Egg laying habitat of the California market squid, Doryteuthis opalescens, appears to be constrained primarily by $\mathrm{pH}_{\mathrm{t}}$ (greater than 7.8) and oxygen (greater than $160 \mu \mathrm{mol})$, though these factors vary much more substantially in the California coastal system than they do off of Massachusetts (Navarro et al. 2018).

While $D$. pealeii has a long history as a research subject, primarily in neuroscience, the dynamics of its physiology under stress have primarily been examined in short-term studies within a breeding season (Kaplan et al. 2013; Zakroff et al. 2019). Early studies of hypercapnia in this squid showed adults were capable of surviving exposures up to $31,500 \mathrm{ppm} \mathrm{CO}_{2}$ unless compounded by hypoxia (Redfield and Goodkind 1929). Birk et al. (2018) further demonstrated that juveniles and adults of this species are able to manage metabolically under acidification (1410 ppm). The embryos, conversely, have some sensitivity to acidification, with developmental delays, decreased mantle length, and degraded statoliths being observed in eggs reared at 1300 ppm and above (Kaplan et al. 2013; Zakroff et al. 2019). Zakroff et al. noted, also, shifts in the intensity of these acidification responses across the breeding season (2019). Paralarvae may have even greater sensitivity, with decreases to swimming activity and velocities having been observed at $1000 \mathrm{ppm}$ and above (Zakroff et al. 2018). Here, however, the potential for interannual variability in response was posited, as a markedly reduced ability to maintain vertical station seen in 2013 was not observed in 2014 and 2015 in these experiments (Zakroff et al. 2018).

This study builds off of the experiments run in 2011 and 2013 (Kaplan et al. 2013; Zakroff et al. 2019) and compiles these data with three additional years (2014 - 2016) of 
experimentation with $D$. pealeii eggs reared under chronic acidification to examine these variations in sensitivity observed on seasonal and interannual scales. Data from all years are compiled and presented to examine the range and variability of positive and negative responses with respect to the experimental and natural (observed) environmental conditions. Using response ratios, a technique primarily used in meta-analyses, we focus on responses at one $\mathrm{pCO}_{2}$ treatment (2200 ppm) within four metrics (dorsal mantle length, hatching time, internal yolk volume, and hatching success), all measured within most experiments, to examine variability across years and within the breeding season. Additionally, we report observations of seasonal shifts in the control response/baseline state of the embyros and paralarvae that occur independent of rearing conditions. We tie these observations to parental preconditioning by using local temperatures as a proxy for broad environmental variability. We also discuss the physiological relationships between metrics and their observed response variability and describe what characterizes an acidification-sensitive versus resistant clutch of developing eggs for this squid.

\section{Materials and Methods}

\section{Summary of Experimental System}

Data analyzed here are all from experiments performed at the Environmental Systems Laboratory (ESL) of the Woods Hole Oceanographic Institution, Woods Hole, Massachusetts, USA between the months of May and October over several years (2011, 2013 - 2016), as summarized in Table 1. A concise description of the experiments will be provided here with notable differences highlighted. Detailed methods for years with published data can be found in their corresponding citations (Table 1).

In brief, adult longfin squid, in the best condition possible after being trawled from Vineyard Sound, were acquired from the Marine Biological Laboratory Marine Resources Center and transported to the ESL. Squid were housed (in different sex ratios depending on year/study; 2016 notably only used individual females) in flow-through rounds tanks $(120 \mathrm{~cm}$ diameter, 70 $\mathrm{cm}$ depth) that were maintained with sand-filtered, temperature-controlled water $\left(20^{\circ} \mathrm{C}\right.$ in 2011 , reduced to $15^{\circ} \mathrm{C}$ from 2013-2016 to reduce metabolic stress and damage through infighting; Salinity $\sim 32 \mathrm{psu}, \mathrm{pH}_{\mathrm{nbs}} \sim 7.96$ ) pumped directly from Vineyard Sound. Egg capsules were typically laid in these tanks 2-3 days after transport. D. pealeii deposits eggs in mucosal 
capsules, 50-300 eggs per capsule, which are strung together and bound to benthic substrate or structure (Jacobson 2005). After being laid, eggs capsules were randomly sorted into the treatment cups of the acidification system to initiate a trial (2 per cup in 2011, 2013 - 2015; 1 per cup in 2016).

Experimental cups ( 3 - 4 per treatment) were maintained in a $20^{\circ} \mathrm{C}$ (average temperature for Vineyard Sound from May - October, NOAA Station BZBM3) water bath. An additional cup without egg capsules served as a water-chemistry control. Egg capsules were transferred directly to this temperature in 2011, 2013 - 2015 and the May 13 trial of 2016, but were started at $15{ }^{\circ} \mathrm{C}$ and acclimated $1{ }^{\circ} \mathrm{C}$ per two hours in the remaining trials of 2016. Vineyard Sound seawater (UV-sterilized and $10 \mu \mathrm{m}$ filtered from 2013 onward) was warmed/chilled to $20^{\circ} \mathrm{C}$ prior to entering a header tank in the egg capsule culture room. The seawater was then apportioned to ' $\mathrm{H}$ ' shaped PVC equilibration chambers, within which $\mathrm{CO}_{2}$ gas mixtures were vigorously bubbled (Figure 1B). Early experiments (2011 and Jul 3 trial of 2013) used ambient facility water, bubbled with ambient air, as the control; however, ESL water is slightly acidified compared to its source in Vineyard Sound ( $\sim 550 \mathrm{ppm}$ compared to $400 \mathrm{ppm}$ at source). The system was outfitted with additional equilibration chambers during the Jul 11 trial of 2013, which has no control or ambient treatment, but a low treatment ( 850 ppm; see assumptions below). Equipment necessary to obtain a 400 ppm control was installed for the Aug 7 trial of 2013 and used throughout the remaining experiments.

Treatment seawater entered a PVC manifold and then was fed via tubing to the experimental cups ( $\sim 20 \mathrm{~L} \mathrm{day}^{-1}$ flow per cup). Cups were bubbled with treatment gas mixtures, with the bubbler positioned in front of the mesh outflow window to push hatched paralarvae away from the outflow current. Seawater chemistry for all cups was monitored by regular (every 3 days) measurement $\mathrm{pH}_{\mathrm{nbs}}$, while more robust sampling and measurement for salinity, alkalinity, and spectrophotometric $\mathrm{pH}_{\mathrm{t}}$ was performed every seven days on control cups during the trials. Water bath temperature and room lighting (14:10 light:dark photoperiod) were monitored with data loggers at 15 minute intervals (HOBO pendant model UA-004-64, Onset Data Loggers, Bourne, Massachusetts, USA). Seawater concentrations were then calculated with $\mathrm{CO}_{2} \mathrm{SYS}$ (Pierrot, Lewis, \& Wallace, 2006; Table 1).

\section{Summary of Metrics}


Eggs in the acidification culture system developed for $\sim 14$ days at $20{ }^{\circ} \mathrm{C}$ at low $\mathrm{pCO}_{2}$ treatments. Cups were monitored daily for hatching. Once hatching began, paralarvae were removed from cups, counted, and subsampled for a range of morphological, physiological, and behavioral measurements; not all measures were performed in all trials or all years. This manuscript focuses on four metrics measured across most of the experiments performed: Dorsal mantle length (DML) of hatchling paralarvae anesthetized in $7.5 \% \mathrm{w} / \mathrm{v} \mathrm{MgCl}_{2}$ mixed with equal part seawater and photographed under dissecting scope (Figure 1A); Hatching time (HT), reported here as the time to $50 \%$ hatching, calculated from counts of paralarvae transformed into curves of cumulative percent hatching over time for each cup/treatment (Figure 1B); Yolk sac volume (YV) measured in a subsample of anesthetized and formalin fixed paralarvae stained with oil red $\mathrm{O}$ and calculated from dissecting scope photographs using the methods of Vidal et al. (2002; Figure 1C); Hatching success (HS), which was determined by dissecting egg capsules post-hatch, counting the remaining embryos, and using that number to produce a percentage of hatched to total eggs (Figure 1D). YV and HS were added as metrics in 2013, and so were not measured in 2011. Only DML was measured in the May 22 trial of 2015, and all but YV measured in the Jun 6 trial of 2015.

\section{Summary of Experiments}

The experiments performed in 2011 used just ambient and 2200 ppm treatments to look for an acidification response (Kaplan et al. 2013). In 2013, a range of treatments was chosen as part of determining the threshold dose response of squid eggs to acidification, and the set of metrics examined was expanded (Zakroff et al. 2018, 2019). 2014 was intended to be a set of consistent replicates focused around the threshold(s) uncovered in 2013. Following the unexpected resilience in 2014, 2015 experiments initially repeated 2014 treatments as part of a rapid assessment of responses for the year and then were increased above 2200 as part of determining a new positive control (data not discussed here). Data from 2016 comes from experiments performed in a multifactor design with warming included as an additional stressor; data is from the $20^{\circ} \mathrm{C}$ temperature controls from those trials (Chapter 5 of this dissertation).

Treatments used in each trial of each year are reported in Table 1. All experiments included a low/ambient/control $\mathrm{pCO}_{2}$ and $2200 \mathrm{ppm}$, a value originally expected to be a relatively extreme positive control despite its occurrence on short times scales in nearshore and 
estuarine systems (Baumann et al. 2015; Gledhill et al. 2015), which serve as the focus of this manuscript. Other treatments are used to illustrate trends in metrics over a range of acidification, but do not enter these analyses beyond that.

\section{Response Ratios}

Data are primarily presented here as log-transformed response ratios (RR), a measure of effect size often used in ocean acidification meta-analyses (Kroeker et al. 2010; Harvey et al. 2013; Cattano et al. 2018). Baumann et al., however, used response ratios as a means of quantifying and resolving trends in acidification sensitivity in a single, robustly and serially studied species of fish (2018). Here, we similarly use this measure in a single species, which has been robustly and serially experimented upon. In this case, we examine effects of acidification in a cephalopod, using this metric to examine shifts in sensitivity on large temporal scales, and, further, compare responses between metrics to examine physiological relationships and hypothesize about potential mechanisms of resistance. The means at $2200 \mathrm{ppm}$ and the lowest $\mathrm{pCO}_{2}$ treatment (typically $400 \mathrm{ppm}$ ) for each metric in each trial were used to calculate the response ratio, e.g., $R R=\ln \left(\mathrm{DML}_{2200}\right)$ - $\ln \left(\mathrm{DML}_{\text {Low }}\right)$. Mean $\mathrm{RR}$ 's and 95\% confidence intervals were calculated for each year (using a bias-corrected accelerated bootstrap; bootstrap in arch 4.8.1; ARCH for Python, https://pypi.org/project/arch/). Acidification effects are considered significant if the $95 \%$ confidence interval does not include zero.

\section{Statistics}

Calculation of response ratios, confidence intervals, and all other statistical analyses were performed in Python (version 3.5.5, Python Software Foundation) within a Jupyter Notebook (Project Jupyter). Raw data was visually assessed for normality through quantile plots and histograms (yolk volume data was normalized by log transformation). Normally distributed data (DML, YV, and HT) were then assessed in Type II ANOVAs for shifts in mean as a result of year, trial (nested within year), $\mathrm{pCO}_{2}$, and their interactions. Trends in data are described with linear regressions (LR) with the goodness of fit $\left(\mathrm{R}^{2}\right)$ and $p$ values listed. Higher order $\left(>2^{\text {nd }}\right.$ degree) polynomial trend lines are used as visual aids and are not presented to demonstrate statistical power. Correlations between regressions were assessed with Pearson's correlation coefficient by comparing values from curves at the same set of 200 points (one per day over the 
time period examined). Response ratios for all metrics were scaled and incorporated into a principle components analysis to visually assess differences between years and how metrics and responses drove the separation/grouping of data.

\section{Assumptions}

Early trials of 2011 and 2013 using the ESL ambient $\mathrm{pCO}_{2}$ level ( $\left.\sim 550 \mathrm{ppm}\right)$ as a control, and the Jul 11 trial of 2013, which has a lowest $\mathrm{pCO}_{2}$ of $850 \mathrm{ppm}$, are included as low/control treatments in this analysis. It is assumed to be a reasonable choice on the basis that both response intensities between $2200 \mathrm{ppm}$ and these levels are of similar magnitudes to those at $400 \mathrm{ppm}$ within these sensitive years and Zakroff et al. (2019) found acidification impacts did not observably emerge until around 1300 ppm in 2013. Further, stable equilibrations in the flow through system proved challenging at higher input $\mathrm{CO}_{2}$ concentrations, possibly owing to fluctuations in alkalinity. Calculated equilibrations are reported in Table 1, but are reported and referred to by the concentration of the input gas mixture, $2200 \mathrm{ppm}$, for concision and clarity. While there is some variation from this mark between trials and years, it is assumed to be representative of a substantial amount of acidification across experiments.

\section{Results}

Trends with increasing acidification varied from year to year across metrics, with significant regressions in 2013 and 2016 for decrease in DML and increase in HT (Figure 2). The same trends neared significance in 2011 (Figure 2). Despite clear shifts in the response intensity of DML across years, the interaction between year and $\mathrm{pCO}_{2}$ was not significant for this metric $\left(p>0.05\right.$; Table 2). Both $\mathrm{pCO}_{2}$ alone and trial, nested within year, were significant influences on the DML dataset compiled across years ( $p<0.05$; Table 2). The variance of DML increased slightly with acidification in 2011, increased significantly in 2013, and decrease slightly in all subsequent years of experimentation. Time to $50 \%$ hatching was most strongly influenced by the interaction between year and $\mathrm{pCO}_{2}\left(\omega^{2}=0.735\right)$, although interactions with trial were also influential on this metric (Table 2). Hatching time consistently increased with acidification in all years except 2014. Yolk data were most strongly impacted by the interaction of year with $\mathrm{pCO}_{2}$ treatments $\left(\omega^{2}=0.981\right.$; Table 2$)$. Yolk volume slightly decreased with acidification, nearly significantly in 2015, across years except in 2014, which showed no trend (Figure 2). No 
consistent trends in yolk volume variance were observed across years. Hatching success was always fairly consistently high across acidification in all years, though 2015 is notable for its slightly improved hatches at higher acidification (Figure 2).

Response ratios for $2200 \mathrm{ppm} \mathrm{CO}_{2}$ compiled by year more clearly demonstrate acidification sensitive years (Fig 3). 2011, 2013, and 2016 all showed significant decreases in dorsal mantle length and increases in hatching time (Fig 3). For both metrics, 2013 showed the greatest effect sizes: a 4.9\% (3.9 - 6.2\%) decrease in mantle length and a 9.3\% (5.6 - 11.2\%) increase in time to $50 \%$ hatching. 2014 showed a non-significant $1 \%(0-2.5 \%)$ increase in mantle length at 2200, while 2015 showed a similar near-zero decrease. Hatching time effect size was slightly reversed in 2014 , with a $2 \%$ decrease $(0-6.8 \%)$ in hatching time at $2200 \mathrm{ppm}$. Yolk volume response was negative in all years, but only showed a significant, $24.0 \%(24.0 \%$ $30.6 \%$ ), decrease in 2015 (Figure 3 ). Hatching success was pretty consistently a near zero change, though showed a near significant, 5.0\% (0 - 8.1\%) increase in 2015 (Figure 3). Both in the condensed graph and using the trends imposed on the extended timeline, DML and HT responses appear to reflect each other on an annual scale (Figure 3). YV and HS also show some annual reflection in the condensed graph, but patterns appear more seasonally reflected when individual trials are plotted over time (Figure 3).

Baseline responses at the lowest $\mathrm{pCO}_{2}$ treatments changed over the breeding the season (Figure 4A). DML and YV both fit slightly parabolic curves, decreasing into the summer then slightly rebounding in fall. These curves strongly correlate to the curve for ambient sea surface temperatures parents experienced in Vineyard Sound (DML: r(200) $=-0.93, \mathrm{p}<0.001$; YV: $\mathrm{r}(200)=-0.98, \mathrm{p}<0.001)$. Baseline HT increased significantly over the season, following a linear trend that only weakly correlated to the ambient temperature curve $(r(200)=-0.18, p=$ 0.012). HS slightly, but very weakly decreased across the breeding season; since these data did not fit a regression well it was not compared to the temperature curve.

Relative effect size showed weak trends in all metrics across the breeding season (Fig 4B, C). DML and YV again were slightly parabolic, demonstrating greater negative responses at the tails of the breeding season (Figure 4B). YV in particular, showed a significant decrease in relative effect size toward the end of the breeding season (Figure 4C). HT was weakly linear, with hatching delay effect size increasing slightly with season; when response ratios were compiled into monthly bins, a significant increase in HT response ratio in the August and 
September bin emerged (Figure 4C). HS showed only a weak decreasing trend with season, which may be driven by outlier responses. However, DML, HT, and HS all demonstrated larger confidence intervals in either the early season bin, late season bin or both, indicating that acidification response intensities vary more at the tails of the breeding season.

The principle components analysis did not demonstrate any obvious clustering of sensitive vs. resistant trials or years (Figure 5). Instead of clusters, the data split in a more lamellar way along the axes of the loadings. Years differentiate along the DML/HT axes, while trials vary within a year layer along the YV/HS axes (Figure 5). This pattern fits reasonably well outside of the particularly sensitive trial in 2016, which falls within the response area of the 2013 data.

The patterns emergent in Figure 5 suggested pair-wise relationships between metrics: DML and HT on the scale of year, and YV and HS on the scale of season. Both pairings show significant inverse linear relationships when plotted against each other (Figure 6). Greater decreases of DML correlated with greater increases of HT, with all data points falling within that sector of the plot being from 2011, 2013, and 2016 (shaded box, Figure 6A). Trials that fall within this area also appear to typically have clutches with smaller baseline paralarvae. Greater decreases in yolk volume under acidification corresponded to increased hatching success, with trials falling in this sector coming from all years, but mainly from those where the low treatment paralarvae hatched with more remaining yolk (Figure 6B).

\section{Discussion}

The responses of $D$. pealeii embryos and paralarvae to ocean acidification were strongly influenced by year and season, suggesting influences of both year class and parentage in the conference of sensitivity to offspring. While the raw data demonstrated trends that demarcated 2011, 2013, and 2016 as years containing acidification sensitive clutches, use of response ratios allowed for a much more nuanced examination of temporal trends in metrics and relationships between metrics. A clear shift in OA sensitivities was observed in the response ratio data compiled by year (Figure 3). To our knowledge interannual variability in stress responses has not been described previously in cephalopods, yet such variation has been observed as part of abundance measurements and stock assessments (Yatsu et al. 2000; Pierce and Boyle 2003; Tian 2009; Keyl et al. 2011) and has also been described in the plastic life history strategies of squid, 
which may underlie shifts in population structure (Pecl et al. 2004a; Hoving et al. 2013). As a year class taxa, with a high fecundity, myopsid squids are expected to be quite plastic and adaptable in the face of environmental change (Pecl et al. 2004a; Pecl and Jackson 2008; Guerra et al. 2010; Rosa et al. 2014b; Doubleday et al. 2016). Part of this presumed plasticity stems from inherent variability in offspring size and state, which is thought to serve as a bet hedging strategy against environmental variability (Marshall et al. 2008; Crean and Marshall 2009).

Acidification induced responses to DML appeared to vary primarily on annual scales, although response intensity varied across the breeding season (Figure 3). This link to year is reflected in the relationship of DML with HT (Figure 6A). Hypotheses for these responses in the 2013 data, from potential metabolic suppression or greater spending of the energy budget in homeostasis are discussed in Zakroff et al. (2019). A potential hypothesis for shifting response intensities in DML is that greater responses occur in clutches with larger baseline paralarvae, i.e., they respond more strongly as they have farther to go before reaching their minimum viable size. Examining data compiled across several years, it appears the reverse, clutches that produce smaller paralarvae show greater acidification sensitivity, is instead the case (Figure 6A); although some effect of response intensity variation might be explained by changes to baseline paralarvae size within a sensitive year.

The seasonal shifts in the baseline responses of the embryos and paralarvae, which were all reared at the same temperature $\left(20^{\circ} \mathrm{C}\right)$ and low or atmospheric ambient $(400 \mathrm{ppm}) \mathrm{pCO}_{2}$ treatments, demonstrate the importance of having a solid characterization of the local system for a study species before interpreting experimental findings (Vargas et al. 2017). While a succession of size classes in the adult squid across the breeding season is widely accepted anecdotal knowledge in the Woods Hole scientific community (Arnold et al. 1974; Mesnil 1977; Macy III and Brodziak 2001), these shifts in hatchling paralarval size, yolk volume, and hatching time had not been previously described in this squid to our knowledge. In the cases of DML and YV, quality of paralarvae appears to decrease into summer and then slightly increase again at the end of the breeding season, while for HT, the hatching period appears to just increase over time (again, despite all eggs being reared at the same temperature; Figure 4A). This latter effect presumes some form of inherent shift in the rate of embryogenesis as a result of time of year, perhaps to counteract the increase in development rate as ambient temperatures increase into summer. However, this suggests that late breeding season eggs should be substantially delayed in 
development compared to early season, potentially exposing them to increased fouling or predation.

Seasonal shifts in paralarval size have been seen in the hatchlings of the southern calamari, Sepioteuthis australis, captured in situ, although demonstrating a different curve over the measured period (with peak sizes toward the middle of the sampling period; Steer et al. 2003) than the one found here. Further, this study showed that larger hatchlings of this species were more likely to successfully reach adulthood, indicating that paralarval size is related to likelihood of survival (Steer et al. 2003). Hatchling size is important for prey capture success during the critical period when paralarvae must transition from yolk reserves to hunting (Vidal et al. 2002a). This indicates, then, that survival of hatchlings from this breeding population likely follows a similar trend of decreasing across most of the season and then increasing slightly at its end, and suggests that smaller (OA impacted) paralarvae may have poorer survivorship rates.

Here, YV is a measure of the remaining internal yolk upon hatching, not necessarily the initial input, particularly under acidification stress. A greater amount of remnant yolk in control treatments could be the result of greater initial maternal investment or could be caused by a reduced metabolic rate. Squid do not store lipids, so maternal investment is largely driven by the tradeoff between putting energy into the development of somatic vs. reproductive tissue during growth and the amount of energy captured through feeding prior to egg production (Steer et al. 2004; Moltschaniwskyj and Pecl 2007). Steer et al. (2004) demonstrated that the fecundity and quality of dumpling squid, Euprymna tasmanica, eggs and the rate of embryonic mortality was significantly impacted by food intake, but not temperature. They posited that maternal investment of yolk, as it fuels embryogenesis, is key to the success of the embryos (Steer et al. 2004). This suggests not only that hatchling paralarvae are less likely to survive as the breeding season progresses, due both to smaller sizes and reduced yolk, but also that the total proportion of paralarvae hatched may also follow this trend across the season. This seasonal shift in baseline YV could feed into the response of embryos to acidification, as seen in its relationship with HS, where acidification exposed embryos that consumed more yolk relative to low treatments showed a correspondingly greater proportion of embryos surviving to hatch (Figure 6B). This indicates that egg clutches with greater initial maternal investment are more likely to survive under acidification stress, which could indicate a greater survival potential in early season embryos in this system (Figure 4A). 
Seasonal trends in DML and YV correlated strongly with ambient temperatures (Figure 4A). The relationship between increasing temperature and decreasing size in ectotherms is extremely well characterized (Atkinson 1994). As with other ectotherms, cephalopod growth rates increase with warming, resulting in smaller size at maturity (Leporati et al. 2007; Moreno et al. 2012; Hoving et al. 2013). Temperature has been shown to impact maternal reproductive output and hatching success in Octopus maya (Juárez et al. 2015). The trends seen here, however, are from a scenario where offspring are not being directly exposed to, but are still potentially seeing the effects of ambient temperatures. This could owe to conditioning of the eggs and sperm prior to capture or of the parents themselves (Marshall 2015; Bonduriansky and Crean 2018). Temperature has been shown to induce maternal effects, with the goal that offspring are conditioned to experience the same environment as the parent, a strategy that depends heavily on environmental predictability (Burgess and Marshall 2011). This temperature conditioning both pre- and post-embryonic development may in part explain the succession of size classes seen in Woods Hole, as smallest squids arrive in peak summer (Arnold et al. 1974; Mesnil 1977).

Responses to acidification were strongest and most variable at the tails of the breeding season for several of the metrics examined here (Figure 4B, C). In particular, this was driven by greater sensitivity in the early trials of 2013 and the last trial of 2016. This suggests that rather than a consistent impact of time of year on embryo sensitivity, there is some shift in the state of the spawning population across the season, which can result in either end being sensitive or resistant. This stands in contrast to the differential sensitivities to acidification in warming described in the European market squid, Loligo vulgaris, which was due to the presence of separate and distinct spawning aggregations in winter and in summer (Rosa et al. 2014a). Notably, though, this study saw greater sensitivities in summer progeny, suggesting the potential for greater acidification sensitivity driven by increased temperature history in the parents (Rosa et al. 2014a).

A population succession schema, based on the size class succession noted above, has been proposed for $D$. pealeii in the northwest Atlantic, but has never been robustly quantified or measured (Arnold et al. 1974; Mesnil 1977). In brief, this model proposes that squid comprising the spawning aggregation are primarily hatched in the summer/fall of two years prior at the beginning of the season ( $<2$ years old $)$ and transition to squid hatched from the previous year 
around July/August (<1 year old; Mesnil 1977). There is likely a lot of mixing and variability in this schema, particularly as males and females respond to their environment, and thus mature, differently (Pecl et al. 2004a). It is important to note that the Mesnil (1977) model presents a scenario wherein these squid hatched 2 years prior are upwards of 20 months of age, however ages of $D$. pealeii (sampled broadly across the southern New England region across many years) estimated from statolith increments indicate the standing stock is typically 135 - 180 days old (4 - 6 months), with maximum ages estimated of around 231 days (7.7 months; Macy III and Brodziak 2001). Southern calamari were thought to spawn in one large aggregation, but were shown to be a succession of microcohorts, where newly mature six month old squid were continually coming to maturity and joining the aggregation (Moltschaniwskyj and Pecl 2007). It is possible $D$. pealeii follows a similar model. There is also evidence showing that, despite offshore aggregation of $D$. pealeii over winter, there is some genetic stock separation geographically, which suggests these squid may return to hatching locations or migrate inshore in genetically related groups (Buresch et al. 2006). As such, overwintering environmental history may be relatively consistent among this population of squid, which could potentially serve as a proxy to assess and potentially discriminate year class effects in the acidification responses.

Synthesizing this information with the observations reported here, we could begin to propose and test preliminary hypotheses about what may be driving shifts in acidification sensitivity on interannual scales. For the sake of investigation, we will consider the Mesnil (1977) model wherein the breeding aggregation of D. pealeii in Vineyard Sound within in any year is the result of a combination of squids hatched two and one year prior. Assuming that temperature serves as the most direct influence on parental condition and conferred sensitivity, we can presume that the environmental history of those squid can very broadly be characterized by the two-year average temperature anomaly for the previous two winters (Dec - Feb; Figure 3). These data are not necessarily available for locations where these squid aggregate in the deep waters of the continental shelf, but the regional impact for each year can be assessed from local data (NOAA buoy BZBM3, in this case). It should be clear that there is some potential error in this thought exercise, but keeping those assumptions in mind, we can plot metric response ratios against these two year average overwinter anomalies (Figure S1). Doing so resolves weak linear relationships between both DML and YV and overwintering temperatures (DML: $\mathrm{R}^{2}=0.514, p$ $\left.=0.173 ; \mathrm{YV}: \mathrm{R}^{2}=0.591, p=0.231\right)$. These data suggest the possibility that anomalously warm 
winters result in greater reductions to mantle length under acidification and reduced yolk consumption (a potential indicator of less initial yolk and thus reduced hatching success).

This conceptual model could also be taken a step further by accepting the assumptions of the proposed $D$. pealeii population structure: use two-year overwinter anomaly values for squid laid from May - July, and one-year for squid laid from August onward. Under this hypothesis, there is a significant correlation between overwintering temperature anomaly and mantle length response to acidification $\left(\mathrm{R}^{2}=0.505, p=0.001\right.$; Figure $\left.\mathrm{S} 2\right)$. In addition, there is a weak, but near significant decreasing trend in hatching success with increasing overwinter anomaly $\left(\mathrm{R}^{2}=0.268\right.$, $p=0.058$; Figure S2). Here again, our metrics demonstrate their paired inverse relationships (DML and HT, YV and HS), suggesting related physiological mechanisms in these responses. Overwinter temperature anomaly may be a proxy for a number of impacts to the squid population, such as metabolic stress or trophic cascades, but regardless, under this framework, parental conditioning due to environmental history results in differential sensitivities to acidification.

This thought exercise partially illustrates the dearth of data still needed to resolve the mechanisms underlying these large-scale temporal shifts in squid offspring sensitivity to OA. Mechanisms for parental conditioning and epigenetic transfer are not well described in cephalopods, but have been outlined as a research goal (Albertin et al. 2012; Xavier et al. 2015). Key features such as the development of the germline have not yet been described (the closest relative in which it has is an oyster, Crassostrea gigas; Fabioux et al. 2004a, b). Molecular flexibility in cephalopods is becoming more established, with some work on DNA methylation work having been done in Octopus vulgaris and RNA-editing becoming a prominent and better understood feature of cephalopod adaptability (Albertin et al. 2015; Rosenthal 2015; Alon et al. 2015; Liscovitch-Brauer et al. 2017; García-Fernández et al. 2017). Transgenerational responses are also challenging to study in cephalopods due to the challenging nature of their culture, but are potentially on the horizon with improvements to culture systems and the establishment of model cephalopods (Lee et al. 2009; Vidal et al. 2014). Future cephalopod stress response studies may also be served by linking in assessments of parentage, parental condition, and/or lipid content of the eggs (Buresch et al. 2001; Steer et al. 2004).

This research uncovered novel patterns of interannual and seasonal variability in the response of $D$. pealeii eggs to ocean acidification, which appear to be driven by interactions of 
parentage, year class, environmental history, and seasonal temperatures. Impacts seen in Kaplan et al. (2013) and Zakroff et al. (2019) were valid observations within the context of the sampled time period, but are not, even together, representative of the full potential for sensitivity and resistance in this organism. Only by running experiments with shared treatments over a longer period do we start to resolve patterns of variability and construct a platform from which to hypothesize about mechanisms underlying population response to acidification stress (Browman 2016; Baumann et al. 2018). This work serves as a reminder that organisms are often much more dynamic than a single experiment can quantify and, hopefully, provides support for more replication and long-term studies in the future as we work to resolve organismal responses to global ocean change.

\section{List of Abbreviations}

DML - Dorsal mantle length

ESL - Environmental Systems Laboratory

HS - Hatching success

HT - Time to $50 \%$ hatching

LR - Linear regression

MBL - Marine Biological Laboratory

OA - Ocean acidification

RR - Response ratio

YV - Yolk volume

\section{Funding}

These analyses were based on research supported by National Science Foundation Grant No. 1220034 to TAM. Further support was provided by the National Science Foundation Graduate Research Fellowship under Grant No. 1122374 to CZ.

\section{Acknowledgments}

Thanks to D. Remsen, the staff of the MBL Marine Resources Center, and the crew of the MBL Gemma for their years of help in the capture of squid. Thanks also to R. Galat and the WHOI facilities staff who aided in the construction, maintenance, and support of the ocean acidification 
system at the ESL. D. McCorkle, A. Cohen, M. Kaplan, and M. White designed and ran the 2011 experiments and provided insight on future experiments. D. McCorkle, M. White, E. Bonk, K. Hoering, and J. Rheuban all provided support with seawater chemistry monitoring and measurement. KYK Chan and A. Maas provided input and guidance on OA studies. Research assistants A. Schlunk, C. Wirth were instrumental in the success of experiments across years, as were the contributions of interns and guest students M. A. Lee, D. Weiler, J. Wingar, and L. Fitzgerald.

\section{Bioethics}

All applicable international, national, and/or institutional guidelines for the care and use of cephalopods were adhered to. 
Table 1. Summary of squid egg ocean acidification experiments compiled in this study with abbreviated equilibrated $\mathrm{pCO}_{2}$ data, written as mean (standard deviation), for treatments used in response ratios. Calculated seawater $\mathrm{pCO}_{2}$ values in Kaplan et al. 2013 are reported as an average for each treatment across trials, and error is not given.

\begin{tabular}{|c|c|c|c|c|c|}
\hline Year & $\begin{array}{c}\text { Trial } \\
\text { (Lay Date) }\end{array}$ & $\mathrm{pCO}_{2}$ Treatments $(\mathrm{ppm})$ & $\begin{array}{l}\text { CO2SYS Calculated } \\
\text { Lowest } \mathrm{pCO}_{2}(\mathrm{ppm})\end{array}$ & $\begin{array}{l}\text { CO2SYS Calculated } \\
2200 \text { ppm pCO } \text { pCpm) }_{2} \text { (ppm }\end{array}$ & Publication/Chapter \\
\hline 2011 & $\begin{array}{l}\text { Jul } 2 \\
\text { Jul } 12\end{array}$ & $\begin{array}{l}\text { ESL Ambient, } 2200 \\
\text { ESL Ambient, } 2200\end{array}$ & 626 & 2440 & Kaplan et al. 2013 \\
\hline 2013 & $\begin{array}{l}\text { Jul } 3 \\
\text { Jul } 11 \\
\text { Aug } 7\end{array}$ & $\begin{array}{l}\text { ESL Ambient, } 1300,2200 \\
850,1300,2200 \\
400,1900,2200\end{array}$ & $\begin{array}{l}565.68(43.90) \\
987.43(20.30) \\
488.58(10.50)\end{array}$ & $\begin{array}{l}2199.56(173.47) \\
2380.50(70.62) \\
2130.17(40.31)\end{array}$ & $\begin{array}{l}\text { Zakroff et al. 2018, } \\
\qquad 2019 \\
\text { Chapters } 2 \text { \& } 3\end{array}$ \\
\hline 2014 & $\begin{array}{l}\text { May } 26 \\
\text { Jul } 2 \\
\text { Jul } 18 \\
\text { Sep } 6\end{array}$ & $\begin{array}{l}400,1500,1700,2200 \\
400,1000,1600,2200 \\
400,1000,1600,2200 \\
400,1000,1600,2200\end{array}$ & $\begin{array}{l}471.27(28.31) \\
439.52(58.83) \\
439.87(24.74) \\
418.25(31.45)\end{array}$ & $\begin{array}{l}2001.40(103.08) \\
1787.99(282.04) \\
2033.82(136.79) \\
1909.49(148.80)\end{array}$ & $\begin{array}{l}\text { Zakroff et al. } 2018 \\
\text { Chapter } 3\end{array}$ \\
\hline & May 22 & $400,1000,1600,2200$ & $454.10(51.43)$ & $1770.29(97.62)$ & \\
\hline 2015 & $\begin{array}{l}\text { Jun } 6 \\
\text { Jul } 19 \\
\text { Jul } 29\end{array}$ & $\begin{array}{l}400,1000,1600,2200 \\
400,2200,3500,4800 \\
400,2200,3500,4800\end{array}$ & $\begin{array}{l}408.61(26.66) \\
424.37(40.05) \\
393.03(37.62)\end{array}$ & $\begin{array}{c}1680.39(79.07) \\
1716.99(115.18) \\
1857.39(153.39)\end{array}$ & Unpublished \\
\hline & May 13 & $400,2200,3500$ & $432.60(15.40)$ & $1907.51(24.84)$ & \\
\hline 2016 & $\begin{array}{l}\text { Jun } 19 \\
\text { Jul } 28 \\
\text { Sep } 14\end{array}$ & $\begin{array}{l}400,2200,3500 \\
400,2200,3500 \\
400,2200,3500\end{array}$ & $\begin{array}{l}474.96(17.70) \\
426.05(46.27) \\
387.25(28.31)\end{array}$ & $\begin{array}{c}2005.07(53.17) \\
1856.25(138.93) \\
1908.70(88.79)\end{array}$ & Chapter 5 \\
\hline
\end{tabular}


Table 2. Type II ANOVAs for normally distributed metrics. Yolk volume data was log-transformed for normalization. Significant $p$ values $(<0.05)$ are bolded.

\begin{tabular}{|c|c|c|c|c|c|}
\hline Source & SS & df & $F$ & $P$ & $\omega^{2}$ \\
\hline \multicolumn{6}{|l|}{ Mantle Length } \\
\hline Year & 0.001 & 4 & 0.016 & 0.999 & -0.001 \\
\hline $\mathrm{pCO}_{2}$ & 0.830 & 9 & 5.155 & $<0.001$ & 0.006 \\
\hline Year : Trial & 0.638 & 15 & 2.376 & 0.004 & 0.003 \\
\hline Year : $\mathrm{pCO}_{2}$ & $-1.02 * 10^{-10}$ & 36 & $-1.58^{*} 10^{-10}$ & 1.000 & -0.006 \\
\hline Year : Trial : $\mathrm{pCO}_{2}$ & 0.841 & 135 & 0.348 & 0.706 & -0.015 \\
\hline Residual & 105.4 & 5887 & & & \\
\hline \multicolumn{6}{|l|}{ Hatching Time } \\
\hline Year & -0.214 & 4 & -0.187 & 1.000 & -0.000 \\
\hline $\mathrm{pCO}_{2}$ & 10.62 & 9 & 4.126 & $<0.001$ & 0.011 \\
\hline Year : Trial & 31.86 & 15 & 7.427 & $<0.001$ & 0.033 \\
\hline Year : $\mathrm{pCO}_{2}$ & 718.9 & 36 & 69.83 & $<0.001$ & 0.735 \\
\hline Year : Trial : $\mathrm{pCO}_{2}$ & 189.6 & 135 & 4.911 & $<0.001$ & 0.194 \\
\hline Residual & 27.74 & 97 & & & \\
\hline \multicolumn{6}{|l|}{ Yolk Sac Volume } \\
\hline Year & 3.399 & 3 & 3.527 & 0.029 & 0.000 \\
\hline $\mathrm{pCO}_{2}$ & $-5.26^{*} 10^{-10}$ & 9 & $-1.82 * 10^{-10}$ & 1.000 & -0.000 \\
\hline Year : Trial & -0.892 & 12 & -0.231 & 1.000 & -0.000 \\
\hline Year : $\mathrm{pCO}_{2}$ & $7.578^{*} 10^{4}$ & 27 & 8738 & $<0.001$ & 0.981 \\
\hline Year : Trial : $\mathrm{pCO}_{2}$ & 122.4 & 108 & 3.527 & 0.029 & 0.001 \\
\hline Residual & 1314 & 4092 & & & \\
\hline
\end{tabular}




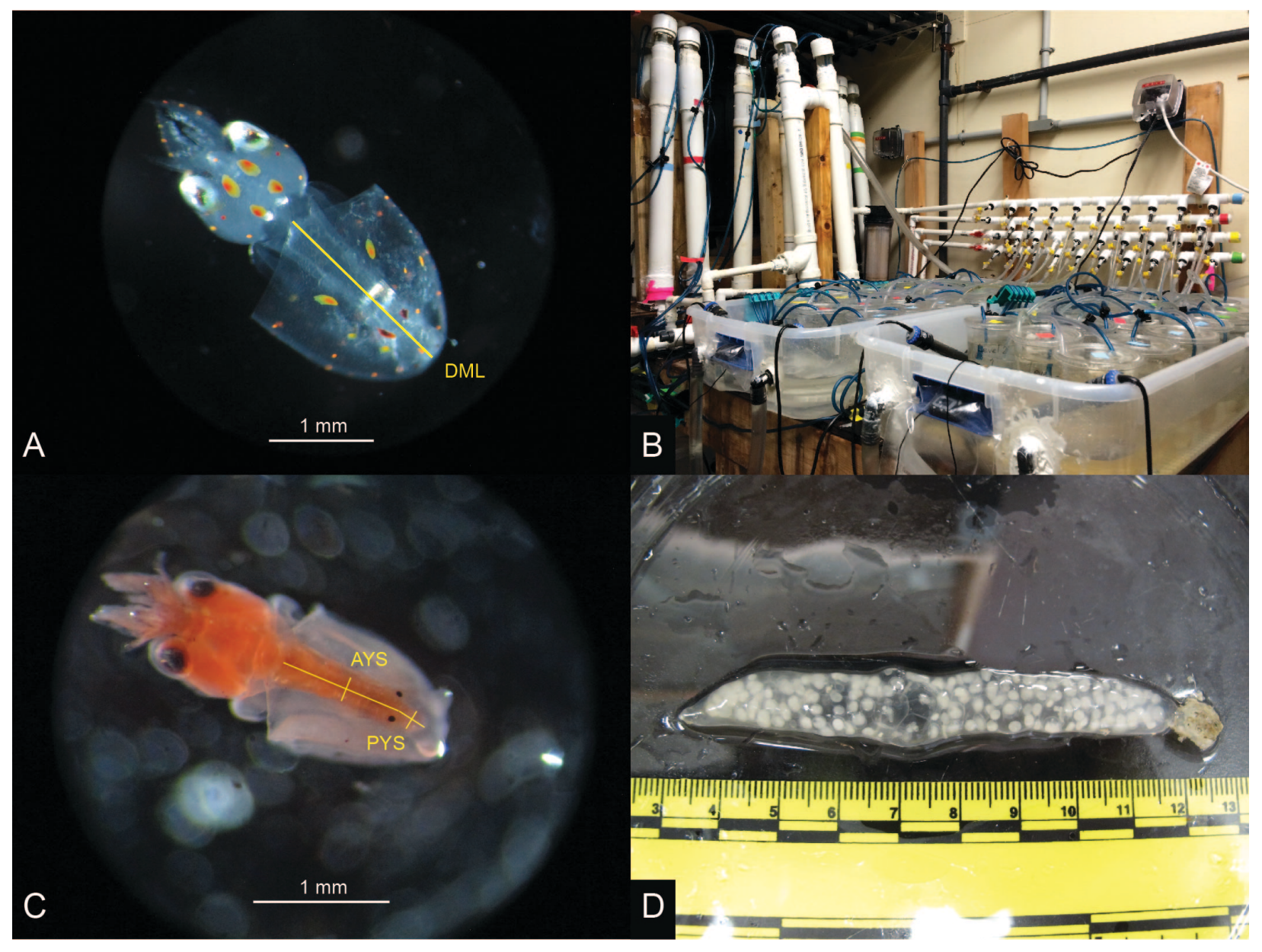

Figure 1. Visual summary of metrics measured across acidification experiments. (A) Mantle Length. A typical paralarva anesthetized and photographed under a dissecting microscope. The yellow superimposed line measures the dorsal mantle length (DML). A $1 \mathrm{~mm}$ white scale bar is at the bottom of the image. (B) Hatching Time. The acidification and squid egg culture system, comprised here, in 2015 , of two $20^{\circ} \mathrm{C}$ water baths (potential for two simultaneously run trials) each containing four $\mathrm{pCO}_{2}$ treatments (color tape marking on cups and $\mathrm{PVC}$ ) with four cups per treatment ( 3 with egg capsules, one without). The white PVC towers are ' $\mathrm{H}$ ' shaped equilibration chambers within which air stones rigorously bubbled flow-through seawater with gas mixtures; equilibrated seawater fed into the manifold at center right, from which drip lines (clear tubing) go to individual cups. The blue tubing comprises gas lines to equilibration chambers and cups. Squid eggs were cultured under flow-through, acidified water in this system until hatching, at which point paralarvae were counted and subsampled for morphometrics. (C) Yolk Sac Volume. A fixed squid paralarva stained with oil red $\mathrm{O}$ to measure yolk sac volume. Yellow lines indicate measurements of the length and width of the interior yolk stores. The anterior yolk sac (AYS) was modeled as either a cone or cylinder (cylinder, here), while the posterior yolk sac (PYS) was modeled as an ellipsoid. A $1 \mathrm{~mm}$ white scale bar is at the bottom of the image. (D) Hatching Success. A single egg capsule removed from the system after hatching had concluded, photographed prior to dissection for counting and categorical staging of remaining embryos 
Figure 2. Trends in acidification response for all metrics across all years of experiments. Data are presented as differences from trial mean to account for shifting baseline size of paralarvae over the breeding season and allow for comparison of relative responses across trials within a year. Yolk volume data was log-transformed to normalize data; differences are not backtransformed. Symbols represent means and bars show one standard deviation. Symbol shape and column title indicate year, while symbol shade indicates trial date, denoted by the date the eggs were laid (see legend at bottom left; earlier trials dark, later trials light). Lines represent linear regressions, with $\mathrm{R}^{2}$ and $p$ value reported at the top of each graph. Significant $p$ values $(\alpha=0.05)$ are marked with an asterisk 


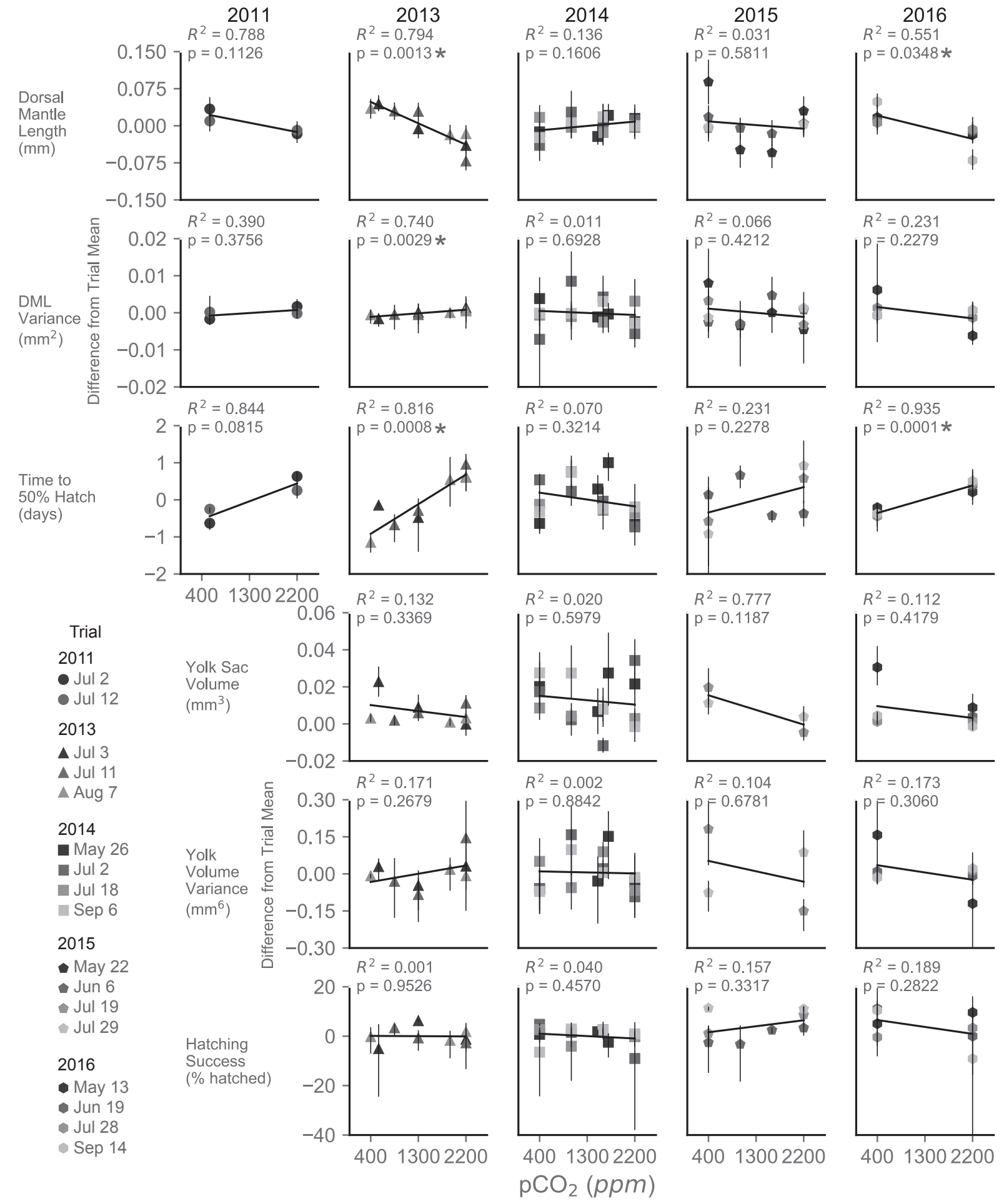




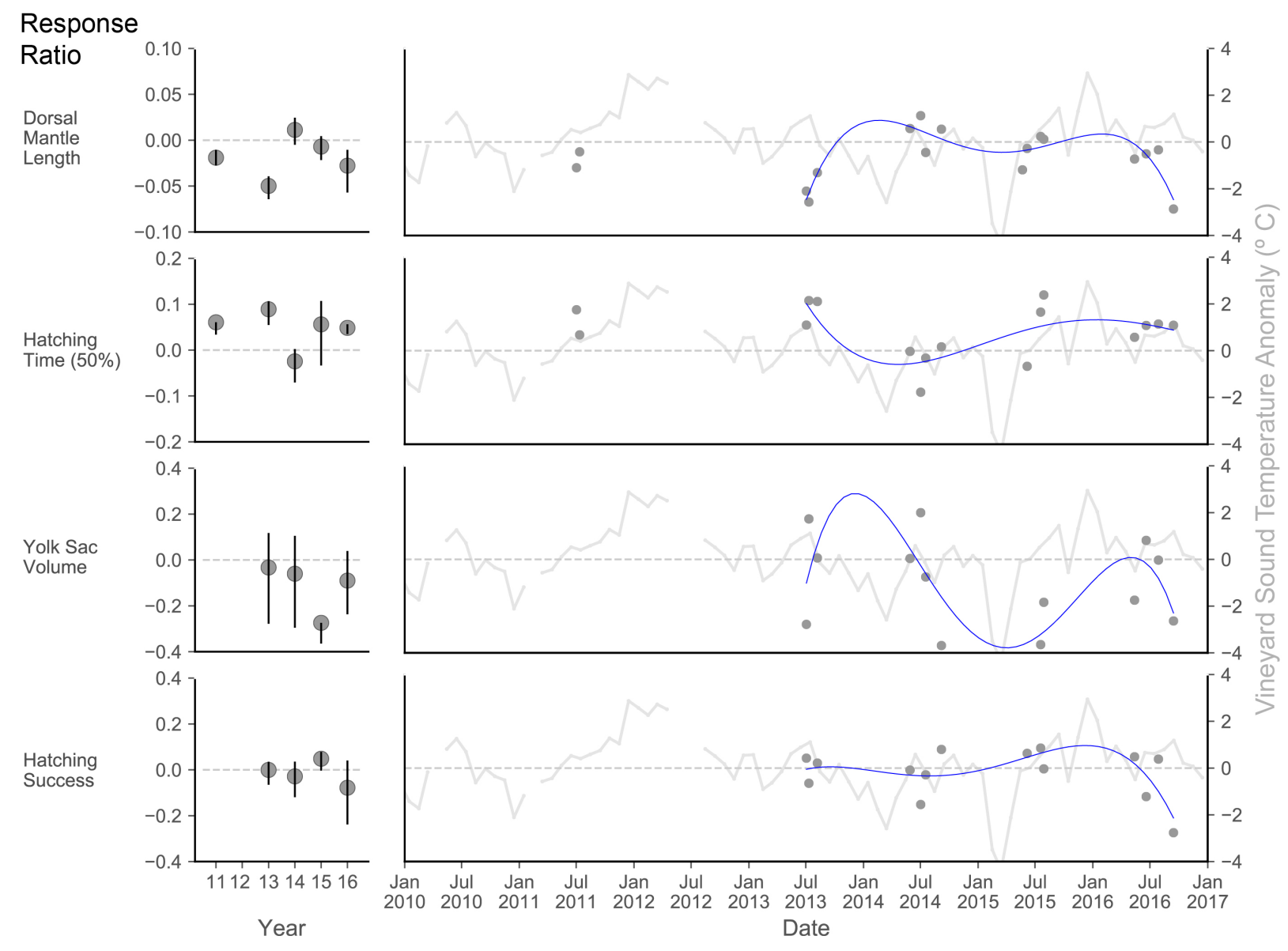

Figure 3. Log-transformed response ratios for each metric. Data are presented at left as mean (symbol) and 95\% confidence interval (bar) for each year. The extended graphs to the right denote the response ratio for each individual trial (symbol). The blue curves are polynomial regressions, to act as visual aids for comparing trends; they are not intended to demonstrate statistical power. The gray line graph depicts the sea surface temperature anomaly $\left({ }^{\circ} \mathrm{C}\right)$ for Vineyard Sound over the depicted period (data from NOAA buoy BZBM3) 
Figure 4. (A) Relative change in baseline (lowest $\mathrm{pCO}_{2}$ value for the trial) response for each metric in the embryos/paralarvae over the breeding season. Data are presented as difference from their year's mean to normalize for differences across years and focus on changes across the breeding season. Symbols represent mean differences. Error is not shown for visual clarity. Shapes correspond to year, but are not shaded by trial, as the $\mathrm{x}$-axis resolves trial order and lay date. Black curves are the best-fit (by $\mathrm{R}^{2}$, presented in plot) regressions, with corresponding $p$ values presented. Significant $p$ values $(\alpha=0.05)$ are marked with an asterisk. The gray curve is the sea surface temperature for Vineyard Sound, MA, over the squid breeding season, averaged across the years of study (2011-2016). (B) Relative change in log-transformed response ratio at $2200 \mathrm{ppm}$ over the breeding season. Data are the differences in log-transformed response ratio for each trial from the mean of all response ratios for that trial's year, to normalize for difference across years and focus on seasonal trends. Symbols, curves, and related statistics are as in (A). (C) Relative response data from (B) binned by trial months into early season (May and June), mid season (July), and late season (August and September) bins. Symbols represent means and bars represent $95 \%$ confidence interval. Lines are linear regressions with corresponding fit and $p$ value presented in plot 
A

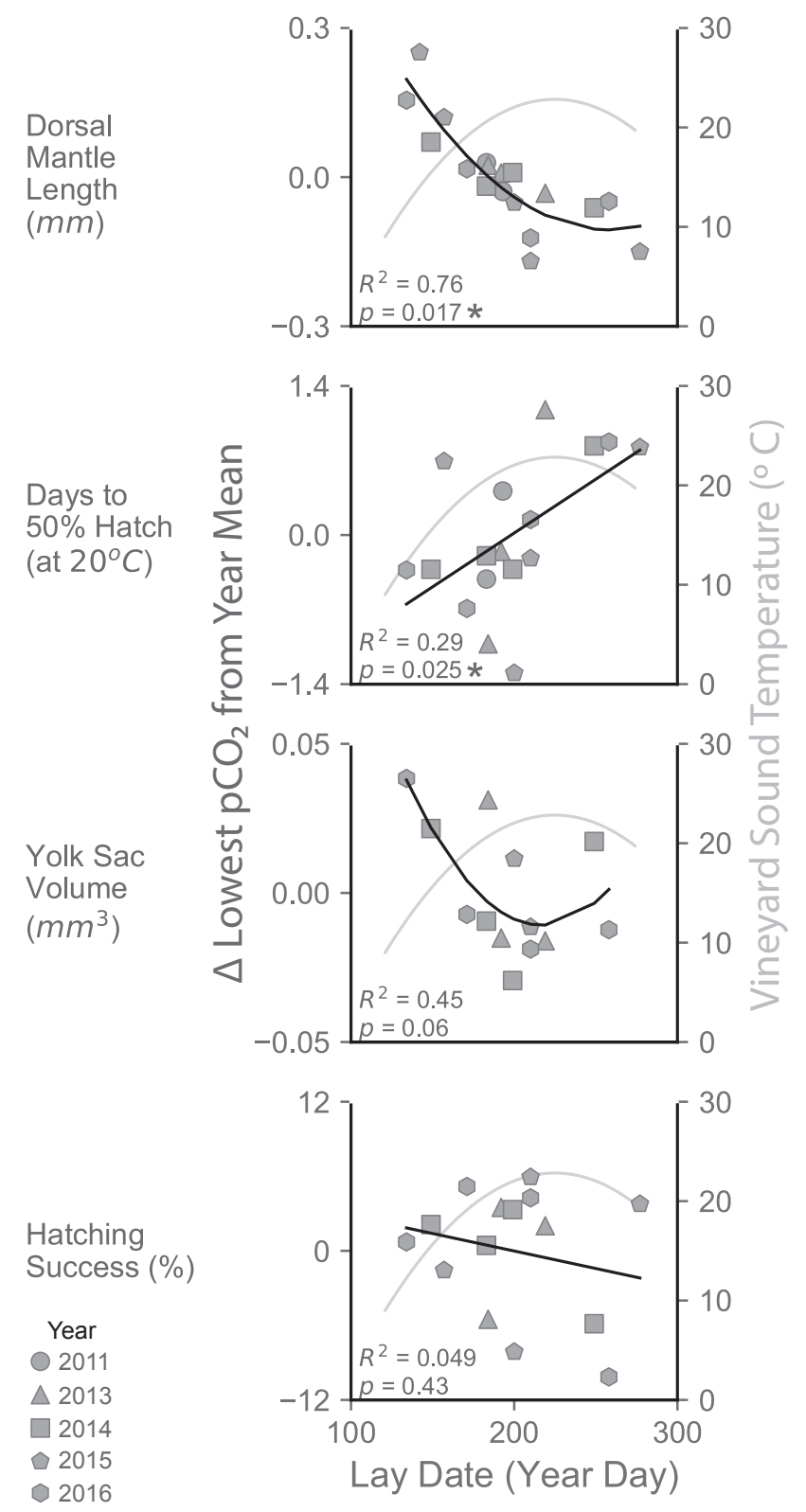

B

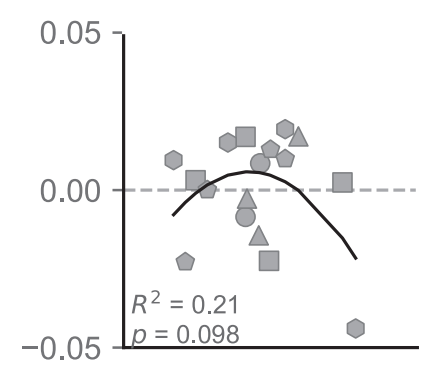

C
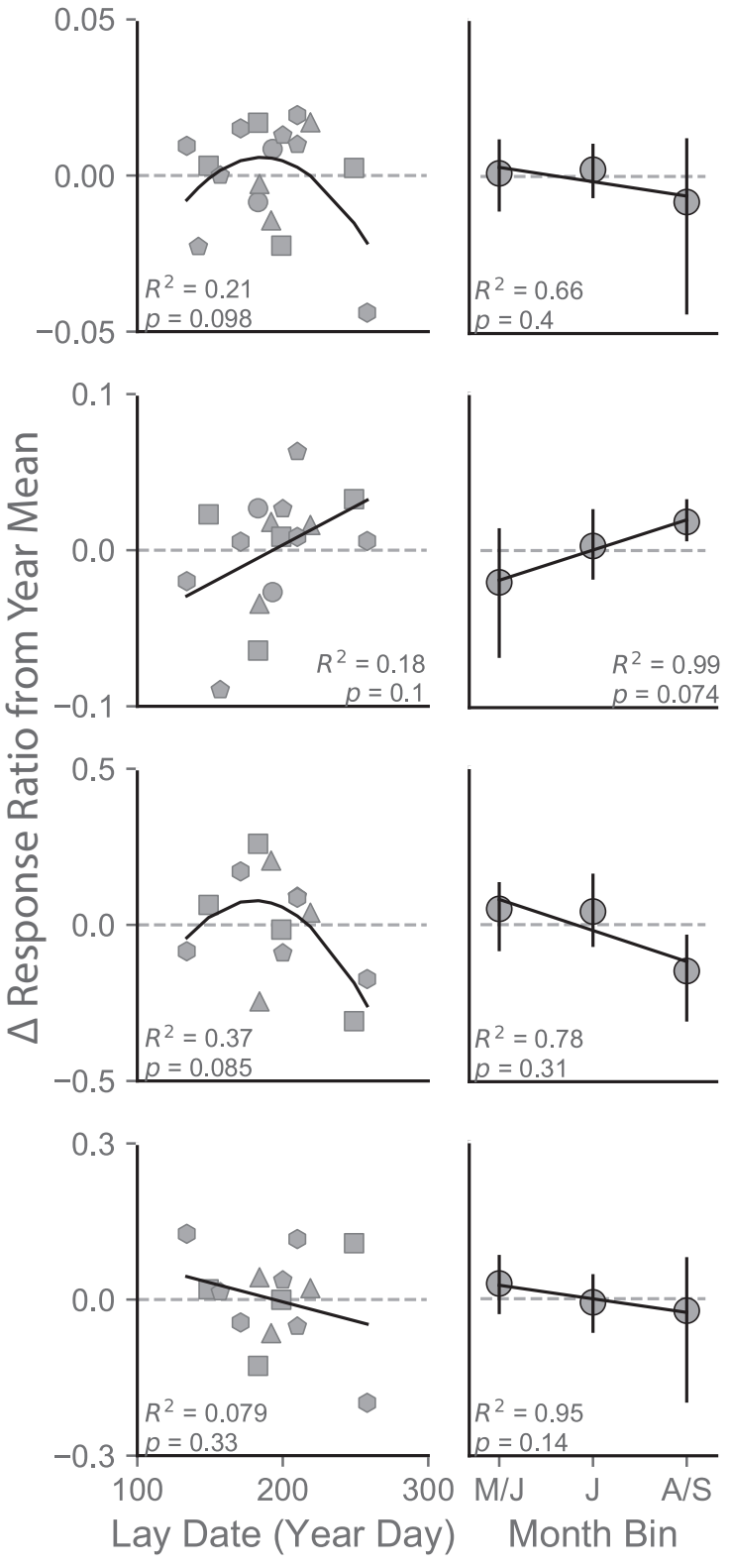


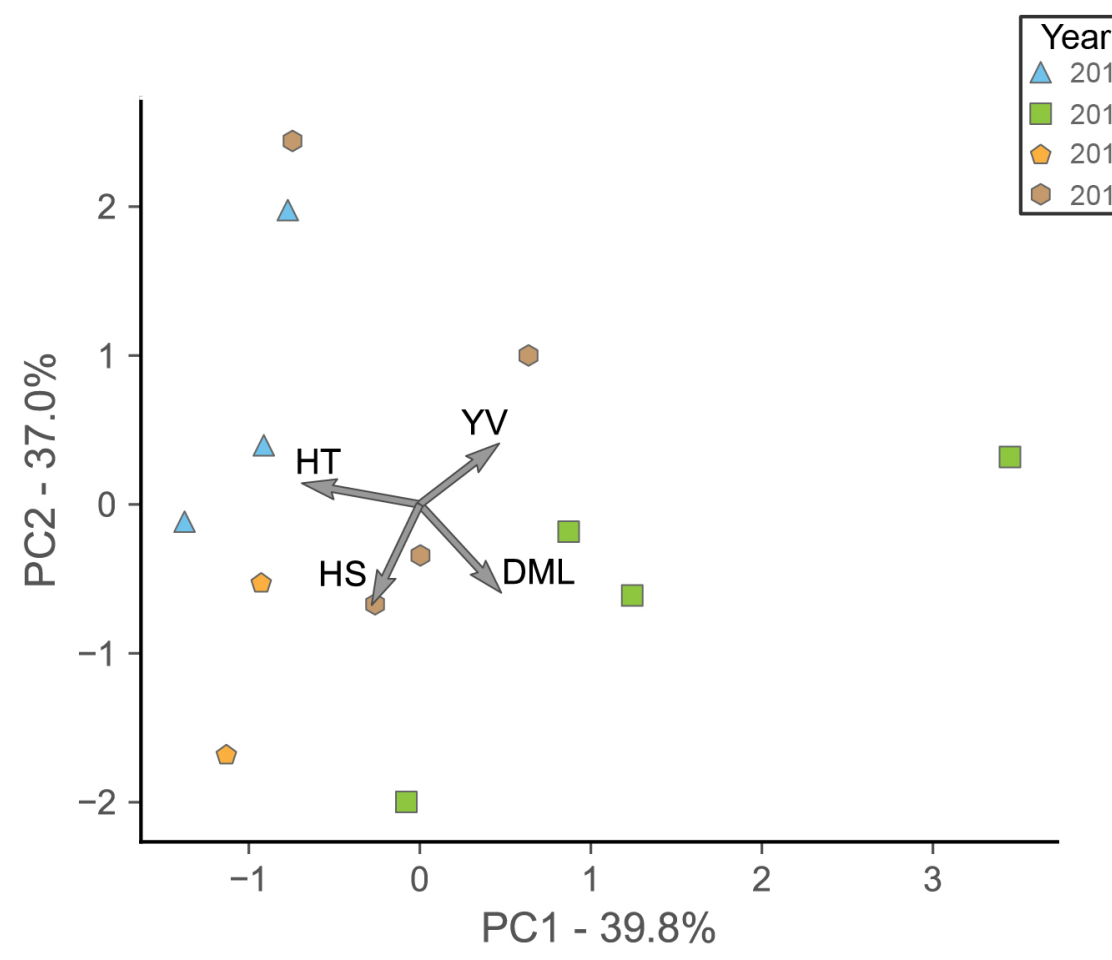

Figure 5. Principle component's analysis using log-transformed response ratios for each metric (for only those trials where all four metrics were measured). Symbols represent principal component value along the first and second components, while shape and color represent year. Axes of variable contribution are imposed to show how metrics (DML: Mantle Length; HT: Hatching Time; YV: Yolk Volume; HS: Hatching Success) influence spread along the PCA axes of variation 


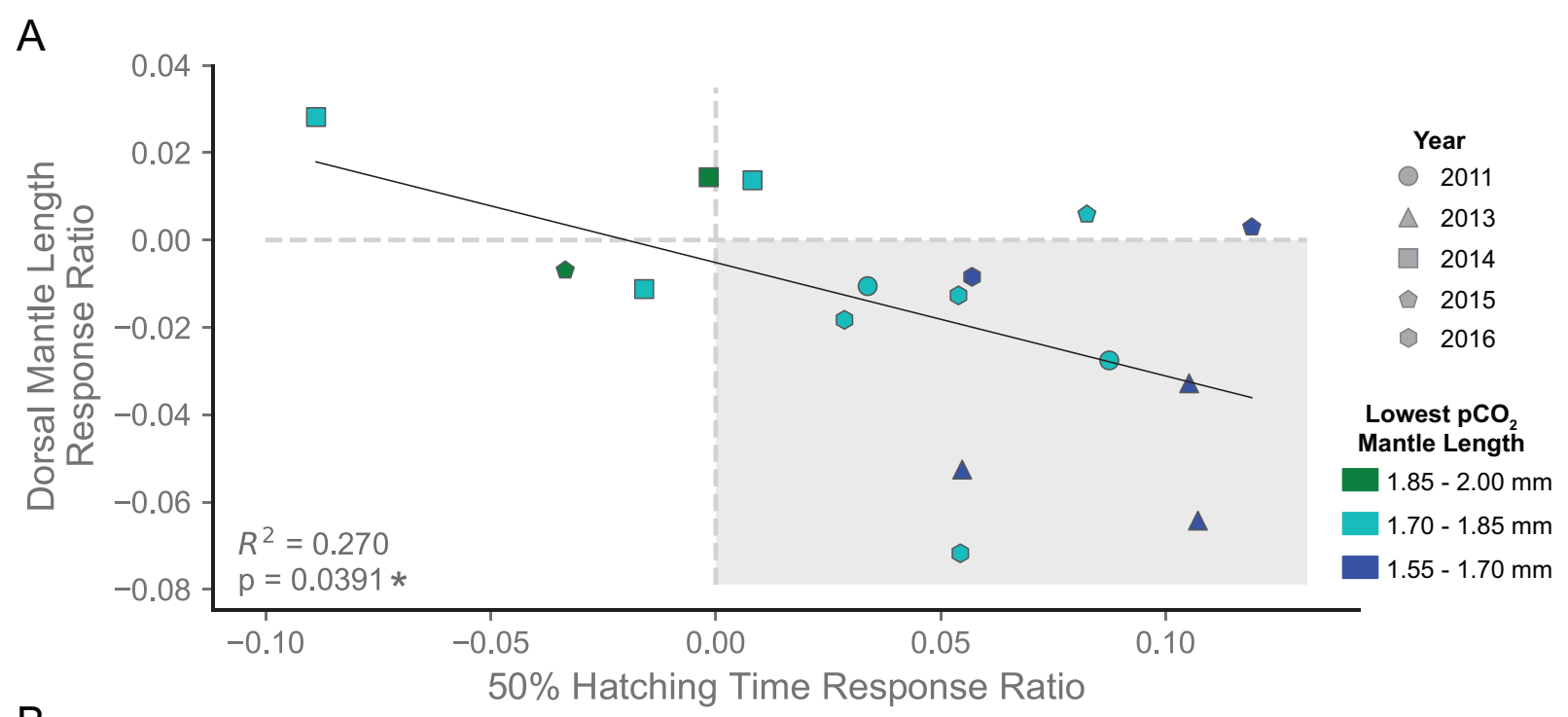

B

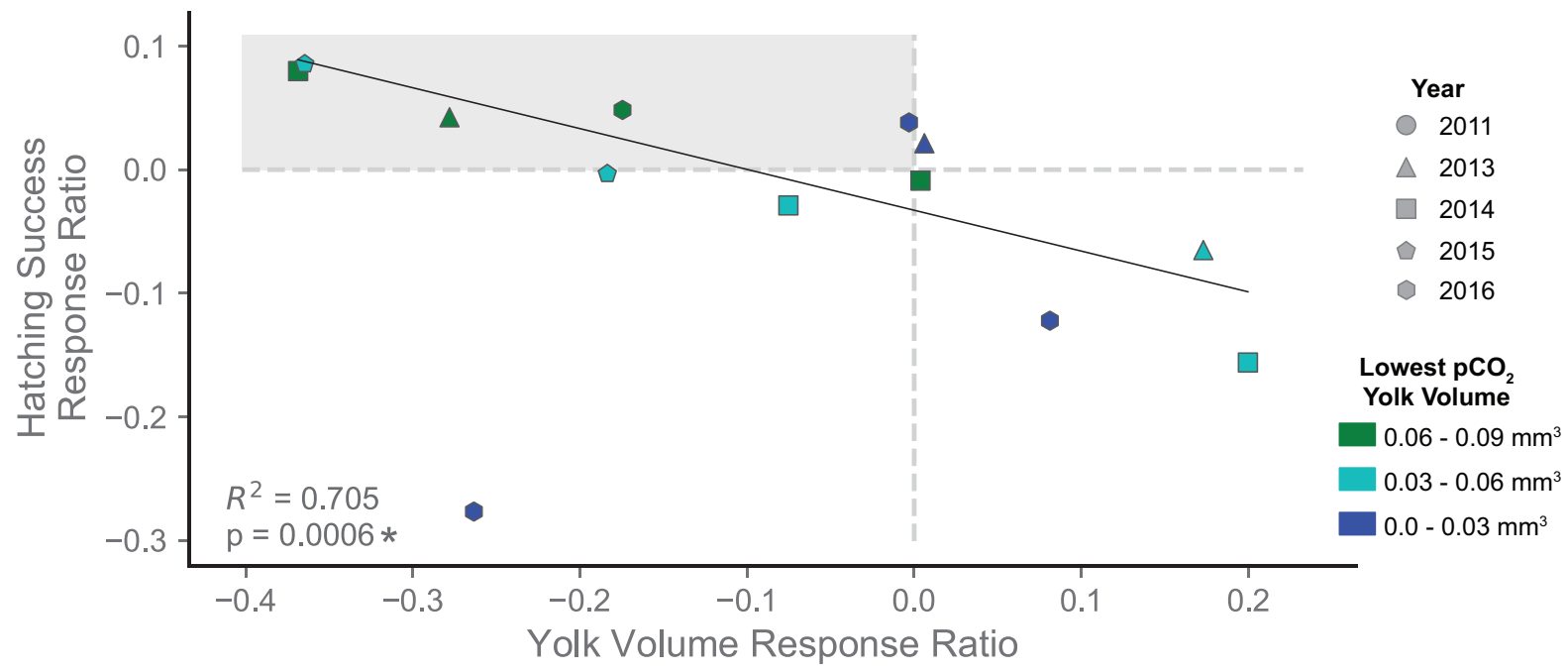

Figure 6. Scatterplots comparing log-transformed response ratio for each trial between metric pairs: (A) Dorsal mantle length vs. hatching time; (B) Hatching success vs. yolk sac volume. In both plots, symbols represent response ratio values for each trial, with the shape of symbol representing the year. Color represents binned values of (A) dorsal mantle length and (B) yolk volume measured in paralarvae from the lowest $\mathrm{pCO}_{2}$ treatment from each trial. Gray boxes are drawn for regions of interest responses: decreased DML and increased HT for sensitive years in (A), decreased yolk volume and increased hatching success in (B). Linear regressions of the data are presented in both plots with corresponding fit and $p$ values presented in the bottom left corner. Significant $p$ values $(\alpha=0.05)$ are marked with an asterisk 


\section{Chapter 5}

\section{Antagonistic interactions and clutch-dependent sensitivity induce variable responses to ocean acidification and warming in squid (Doryteuthis pealeii) embryos}

This chapter is a pre-print of a submitted manuscript, authored by Zakroff, CJ and Mooney, TA, to a special issue of Frontiers in Physiology: Invertebrate Physiology: Cephalopod Research Across Scales - Molecules to Ecosystems. The Supplementary Materials for this chapter can be found in Appendix D. 


\begin{abstract}
Ocean acidification (OA) and warming seas are significant concerns for coastal systems and species. The Atlantic longfin squid, Doryteuthis pealeii, a keystone of the Northwest Atlantic trophic web, has demonstrated impacts under high chronic exposure to acidification (2200 ppm), but the combined effects of OA and warming have not been explored in this species. In this study, D. pealeii egg capsules were reared under a combination of acidification levels (400, $2200, \& 3500 \mathrm{ppm})$ and temperatures $\left(20 \& 27^{\circ} \mathrm{C}\right)$. Hatchlings were measured for a range of metrics (mantle length, yolk volume, malformation, and hatching success) in three trials over the 2016 breeding season (May - Oct). Although notable resistance to stressors was seen, highlighting variability within and between clutches, reduced mantle lengths and malformation of the embryos occurred at the highest OA exposure. Surprisingly, increased temperatures did not appear to exacerbate OA impacts, although responses were variable. Rather, high OAexposed hatchlings from the warmer conditions often showed reduced impacts compared to those reared in ambient temperatures. This may be due to the increased developmental rate and subsequently reduced OA exposure time of embryos in the higher temperature treatment. These results indicate a substantive potential plasticity to multiple stressors during the embryonic development of this species of squid, but do not predict how this species would fare under these future ocean scenarios.
\end{abstract}




\section{Introduction}

Coastal ecosystems are seeing levels of acidification and warming today that are not predicted for the open ocean for hundreds of years (Pachauri and Meyer 2014). Ocean acidification $(\mathrm{OA})$ can be enhanced in coastal regions due to increased $\mathrm{pH}$ variability from freshwater influx, urbanization, and pollution (Gledhill et al. 2015). Rapid coastal warming, particularly in marine hotspots like the northwest Atlantic and the Gulf of Maine region, is already causing substantive impacts to vital marine services and valuable fisheries (Hobday and Pecl 2014; Pershing et al. 2015; Saba et al. 2016). The complexity of this scenario is further compounded by the potential for interactive effects, whether additive, synergistic, or antagonistic, between these stressors for a range of potentially sensitive organisms and processes (Crain et al. 2008; Kroeker et al. 2013; Breitburg et al. 2015). Further, it is becoming clear that while data on global change is limited and generalizations are to some degree necessary, many organismal responses to multistressor scenarios are context-specific to population and/or region (Kroeker et al. 2017). Life stage-specific responses, for example, particularly for early development and dispersal where sensitivities are often thought to be highest, are foundational to understanding how populations may be impacted under multistressor scenarios (Byrne 2011; Haigh et al. 2015).

Coastal squids, the myopsid squids, are a fundamental component of shelf and nearshore food webs, and the longfin inshore squid, Doryteuthis pealeii, takes this place along the northwest Atlantic shelf system (Jacobson 2005). These squid also support a valuable fishery in New England, with landings of 11,000 mt in 2018 (NOAA 2019). These benthopelagic squid overwinter in the deeper, warmer waters of the shelf, but from May to October they aggregate in the shallow nearshore along the northwest Atlantic coastline to breed (Jacobson 2005). They lay their eggs in mucous-bound capsules, which are tied, often in masses, to benthic structures or the substrate (Shashar and Hanlon 2013). These eggs are stationary and must develop under whatever environmental conditions and variability they are exposed to. Observers have recorded eggs laid at depths of up to $50 \mathrm{~m}$, in temperatures of $10-23{ }^{\circ} \mathrm{C}$, and salinities of $30-32 \mathrm{ppt}$, which contextualizes a presumed preferred laying habitat for this species (McMahon and Summers 1971; Jacobson 2005). Less is known about the preferences for $\mathrm{pH}$ of egg laying habitat for this species, but $\mathrm{pH}_{\mathrm{t}}$ calculated from shelf carbonate system profiles throughout their habitat range indicate a typical exposure range of 7.88-8.2 (600-250 $\left.\mathrm{ppm} \mathrm{pCO}_{2}\right)$ during the 
breeding season (Wang et al. 2013; Zakroff et al. 2019). Navarro et al. (2018) reported preferred egg laying habitat for the California market squid, Doryteuthis opalescens, as requiring $\mathrm{pH}_{\mathrm{t}}$ greater than 7.8 and $\mathrm{O}_{2}$ concentrations greater than $160 \mu \mathrm{mol}$ with no apparent temperature limitation within the region. These static ranges do not account for the possible variability of temperature and carbonate system measures across temporal scales, as has been observed in this nearshore system, but at least provide a framework for ideal tolerance windows (Hong et al. 2009; Connolly and Lentz 2014).

Developing embryos are often thought to be particularly sensitive to added stressors because they have limited available energy stores and are actively building the machinery needed to maintain homeostasis (Steer et al. 2004; Hu et al. 2010). This paradigm is challenged, however, by the inherent need for coastal embryos to cope with a highly variable environment and the potential plasticity of an embryo given active and adaptive developmental pathways (Hamdoun and Epel 2007). The question, then, becomes one of the limits of resilience and plasticity during embryonic development, and when human-driven global ocean change will push systems past those limits.

A growing body of research has examined the impacts of various stressors on the early life stages of myopsid squid. Egg capsules of D. opalescens reared under acidification ( $\mathrm{pH} 7.57$, pCO2 1440 ppm) and hypoxia (80 $\mu \mathrm{M} \mathrm{O} 2)$ showed delays to development, potential reductions in yolk volume, and decreases in statolith size relative to the embryo (Navarro et al. 2016). Pimentel et al. (2014) exposed egg capsules of the European market squid, Loligo vulgaris, to warming $\left(+2{ }^{\circ} \mathrm{C}\right.$ above regional seasonal averages) and described a 28 -fold increase in oxygen consumption during embryogenesis, resulting in rapid depletion of available oxygen and causing metabolic suppression in late stage embryos. Further work with L. vulgaris embryos under $+2{ }^{\circ} \mathrm{C}$ warming, removing embryos from the egg capsule, resulted in metabolic suppression that encouraged premature hatching, with an increase in malformations observed among the hatched paralarvae (Rosa et al. 2012). This study also noted that warming-exposed paralarvae, as opposed to encapsulated embryos, activated a stress responses through heat shock proteins and antioxidant enzymes, suggesting the transition to planktonic life could come with the addition of a more robust stress response toolbox (Rosa et al. 2012; Robin et al. 2014). Few studies of warming and acidification have been performed with myopsid squid embryos. Rosa et al. (2014) exposed seasonal clutches of L. vulgaris embryos, removed from their egg capsules, to 
this combination $\left(+2{ }^{\circ} \mathrm{C}\right.$ and $\left.\mathrm{pCO} 2 \sim 1650 \mathrm{ppm}\right)$ of stressors, with summer embryos showing greater sensitivity through: decreased hatching success, increased premature hatching and abnormalities, and acidification-driven delays in development time and decreases in oxygen consumption relative to the increases caused by warming; the effects of removing developing animals from their embroyonic capsule were not determined.

Despite their ecological and economic importance as a myopsid squid, we know little regarding the interactive effects of $\mathrm{OA}$ and temperature on $D$. pealeii. Studies with this species have focused on acidification with early results mirroring those seen in the embryos of $L$. vulgaris and D. opalescens. Kaplan et al. (2013) reared D. pealeii egg capsules under low (550 ppm) and high acidification (2200 ppm), observing development delays of about one day, a decrease in hatchling paralarvae dorsal mantle length, and decreased size and quality of the statoliths. Subsequently, Zakroff et al. (2019) expanded upon this preliminary work, exposing $D$. pealeii egg capsules to a range of $\mathrm{pCO}_{2}$ levels (from 400 to $2200 \mathrm{ppm}$ ), noting delays in development time, decreases in dorsal mantle length, and smaller, rougher statoliths that indicated a potential dose response threshold of $1300 \mathrm{ppm}$. In addition, this study highlighted notable variability in response intensity across the breeding season, demonstrating potentially different physiological strategies or responses to acidification stress within the egg capsules that expressed as different levels of sensitivity or resistance across the season (Zakroff et al. 2019). Further experiments, described in the behavioral studies in Zakroff et al. (2018), showed full years where embryos showed little to no response to acidification even up to the original 2200 ppm level, suggesting greater levels of resilience than had been expected.

In this study, we examined the extent of stress resistance and response variability in developing $D$. pealeii embryos by both increasing the acidification exposure (2200 ppm was considered an exposure level that would elicit a variable response, while $3500 \mathrm{ppm}$ was used as a positive control) and by adding warming $\left(+2{ }^{\circ} \mathrm{C}\right.$ above peak breeding season temperature for Vineyard Sound, MA, USA) as a potentially compounding stressor. Metrics analyzed are the same as those in Zakroff et al. (2019) except that proportions of malformation in the paralarvae were added based on their prevalence in work by Rosa et al. $(2012 ; 2014)$ and statolith data is not included here to maintain concision in this manuscript. Further, unlike most of the previously cited work, which used wild collected or lab produced egg capsules most likely sourced from 
multiple parents, egg capsule maternity was monitored, allowing for an examination of both between and within clutch variability of stress responses.

\section{Methods}

The experiments described here were performed between May and October of 2016 at the Environmental Systems Laboratory (ESL) of the Woods Hole Oceanographic Institution, Woods Hole, Massachusetts, USA during peak breeding season of Doryteuthis pealeii for this region (Arnold et al. 1974; Jacobson 2005). Methods presented throughout are similar to those reported in detail in Zakroff et al. (2019), so will be reiterated in brief, but with differences noted.

\section{Squid capture and care}

Squid were acquired from the Marine Biological Laboratory (MBL) Marine Resources Center from trawls performed at 10-30 meters depth in Vineyard Sound at the Menemsha Bight of Martha's Vineyard. Squid were either selected on ship or following offloading from the ship, but prior to deposition into holding aquaria at the MBL. Females of 15-25 cm dorsal mantle length that exhibited the least signs of stress (calmly resting at bottom or gently hovering with no damage or lesions to fins or skin) and had bright orange accessory nidamental glands were selected. Per trip, three adult females were hand-selected from the catch and each carefully placed into their own seawater filled cooler. The squid were then driven quickly and gently to the

Environmental Systems Laboratory (ESL) at the Woods Hole Oceanographic Institution. Female squid were gently transferred from the coolers into one of three flow-through round tanks (120 $\mathrm{cm}$ in diameter, $70 \mathrm{~cm}$ depth). Overall, time from capture to introduction to the tanks at the ESL was less than six hours from capture to tank.

Each female had her own tank with no males or other squid present. These tanks were fed by Vineyard Sound seawater that had been sand-filtered and cooled to $15^{\circ} \mathrm{C}$ (Salinity $=32 \mathrm{psu}$, $\left.\mathrm{pH}_{\mathrm{nbs}}=7.96\right)$. As noted in Zakroff et al. (2019), this temperature occurs during the breeding season (9.60 - $25.40^{\circ} \mathrm{C}$ from May - October 2016 from NOAA Station BZBM3), but is typically lower than the ambient mean $\left(19.57^{\circ} \mathrm{C}\right.$ from May - October 2016 from NOAA Station BZBM3), and was used to avoid increased damage and stress due to increased metabolism and activity under higher temperatures. Each aquarium had a ca. $2 \mathrm{~cm}$ thick layer of sand at the bottom, was continuously bubbled with air, and was covered throughout the day to avoid startling. Each tank 
included false egg capsules, comprised of the inflated fingers of nitrile gloves, which had been seawater soaked and cleaned, zip-tied to a weight, to encourage egg-laying on a clean surface away from the substrate or air hose. Squid were fed once per day with local killifish, Fundulus heteroclitus. All female squid were fed and maintained in the ESL until they died following egg laying.

New female squid were brought in for each trial. Female squid typically laid egg capsules after two days of capture, producing small mucous-bound mops of around 20-30 egg capsules, each containing 80-300 eggs. These female squid fertilized eggs with stored sperm from breeding that occurred prior to capture, so paternity was unknown (and potentially complex; Buresch et al. 2001), but maternity of all eggs was known. Tanks were checked for eggs each morning. If eggs were present, they were immediately hand-transferred into a clean 5-gallon bucket of $15^{\circ} \mathrm{C}$, filtered seawater and taken to the room containing the egg culturing system. Only egg capsules of high quality (orange-tinted, thin, and oblong fingers with no notable air pockets or other damages) were selected and randomly hand sorted into the experimental system to initiate a trial (described below).

\section{Squid egg culture system: acidification and warming}

Details of the culture and acidification system are the same as those reported in Zakroff et al. (2019). In brief, $15^{\circ} \mathrm{C}, 10 \mu \mathrm{m}$ filtered, and UV-treated Vineyard Sound water was fed into an air-bubbled header tank, which gravity-fed several H-shaped PVC equilibration chambers that each contained four airstones (two per leg) bubbling with gas mixtures (400, 2200, and 3500 ppm $\mathrm{CO}_{2}$ ) for the three acidification treatment lines. Treatment gases were not tested with a $\mathrm{CO}_{2}$ analyzer as they were in Zakroff et al. (2019) because treatments exceeded the range of the meter. Water in the ESL increases in $\mathrm{pCO}_{2}$ compared to environmental ambient, from $400 \mathrm{ppm}$ to about $550 \mathrm{ppm}$, so the water in the $400 \mathrm{ppm}$ control was first degassed with $\mathrm{N}_{2}$ in one $\mathrm{H}$ chamber and then re-equilibrated with ambient air in two subsequent chambers. Given the several stages of holding and filtration input water goes through as it enters both the ESL and the acidification system, it is presumed that it is not subject to small-scale environmental variability due to mixing over time. No such variability was noted in temperature, salinity, or $\mathrm{pH}$, but moderate variability in alkalinity was observed within and between trials (Table 1). 
Acidified water left the equilibration chambers and entered PVC manifolds and was carried by drip lines to the individual experimental culture cups (pre-soaked, 1-liter PET containers, Solo Foodservice, Lake Forest, IL; 5 cups per treatment * 3 treatments per water bath $=15$ cups per water bath). Each cup was sealed with a lid through which a drip line was fed to the bottom of the cup. A bubbling line of the appropriate gas concentration was also fed through the lid to about half way up the cup and aligned underneath the outflow window, so as to not disturb the egg capsule during development, but push hatched paralarvae away from the outflow screen. Water outflowed through a $5 \mu \mathrm{m}$ mesh window in the treatment cups into the surrounding water bath, which then outflowed to the drain.

Each water bath was maintained at $15^{\circ} \mathrm{C}$ by an aquarium chiller (Oceanic Aquarium Chiller 1/10hp, Oceanic Systems, Walnut Creek, California, USA) and heaters (JÄGER 3603, EHEIM GmbH and Co., Deizisau, DE) to match the maternal holding tanks until introduction of eggs for a trial. Although in Zakroff et al. (2019) temperature acclimation between holding tanks and experimental tanks was not performed, as transfer from 15 to $20^{\circ} \mathrm{C}$ showed no impact to the eggs, the shock from 15 to $27^{\circ} \mathrm{C}$ was highly impactful to egg capsule survival in preliminary experiments and so methods were changed to acclimate eggs slowly to temperature. Upon introduction of eggs, water baths were increased in temperature 1 degree every 2 hours until desired treatments temperatures were reached: $20^{\circ} \mathrm{C}$, the average seasonal temperature, and 27 ${ }^{\circ} \mathrm{C}$, two degrees above peak temperature $\left(25^{\circ} \mathrm{C}\right)$ in Vineyard Sound from May - October. At least two water baths were run per trial, although the Jun 18 and Jul 28 trials each had a third temperature treatment $\left(25^{\circ} \mathrm{C}\right.$ and $30^{\circ} \mathrm{C}$ respectively) not reported here. Water bath temperatures were rotated between the Jun 19 and Jul 28 trials (Table 1). Each water bath was monitored with a HOBO data logger (HOBO pendant model UA-004-64, Onset Data Loggers, Bourne, Massachusetts, USA), which recorded temperature and ambient light every 15 minutes. The culture room used ceiling mounted fluorescent lighting, which was set to a 14:10 light:dark photoperiod (broadly that of the natural system during this time).

\section{An experimental trial: egg rearing and monitoring}

Trials were initiated by the presence of eggs in the maternal holding tanks and are demarcated by lay date (Jun 19, Jul 28, and Sep 14). Egg capsules were randomly sorted by hand into four out of the five cups in each treatment (with the last cup acting as an abiotic control for 
monitoring of seawater chemistry). A full trial therefore was comprised of 12 egg capsules per water bath, requiring 24 capsules for two baths or 36 for three. This "full" number of egg capsules was not always reached in each trial, so egg capsules were sorted to prioritize each treatment having as many replicates as possible (see $n$ 's in Table 1). Only the core treatments of acidification $(400,2200$, and $3500 \mathrm{ppm})$ for acclimated temperatures $\left(20\right.$ and $\left.27^{\circ} \mathrm{C}\right)$ consistently repeated across all three trials are reported here.

Following the introduction of eggs and temperature acclimation, water samples were taken from every cup for carbonate system measurements. These methods mirror exactly those described in Zakroff et al. (2019) except that salinity was no longer taken with bottle samples and was instead measured using a salinity probe (Orion Star ${ }^{\mathrm{TM}}$ A329, Thermo Fisher Scientific Inc., Waltham, Massachusetts, USA). Data from spectrophotometric $\mathrm{pH}_{\mathrm{t}}$, alkalinity, salinity, and temperature were input into CO2SYS (Pierrot et al. 2006), calculated with dissociation constants from Mehrbach et al. (1973) and sulfate constants from Dickson (1990), to produce $\mathrm{pCO}_{2}$ values for the seawater treatments (Table 1). These measurements were repeated on just the abiotic control cup every seven days after a trial's initiation (twice more, usually). The $\mathrm{pH}_{\mathrm{nbs}}$ of all cups was measured every three days using a three-point standard calibrated $\mathrm{pH}$ probe (Orion $\mathrm{Star}^{\mathrm{TM}}$ A329, Thermo Fisher Scientific Inc., Waltham, Massachusetts, USA). These pH measurements were used primarily to monitor the stability of the $\mathrm{pH}$ in the system and ensure $\mathrm{pH}$ of the biotic cups did not vary notably from the abiotic controls.

Egg capsules were left to develop undisturbed, with particular care taken during chemical monitoring, within the treatment system. Cups were checked daily to observe development and check for hatchlings. Under ambient $\mathrm{pCO}_{2}$, hatching typically initiated after 13-15 days in the 20 ${ }^{\circ} \mathrm{C}$ temperature control, and 8-10 days in the $27{ }^{\circ} \mathrm{C}$ warming treatment. Each hatching day, all paralarvae were removed, counted, and subsampled for the various measurements described below. All paralarvae not preserved separately as part of a specific analysis were anesthetized with $7.5 \% \mathrm{w} / \mathrm{v} \mathrm{MgCl}_{2}$ mixed with equal part seawater and preserved in $70 \%$ ethanol in microcentrifuge tubes $(0.65 \mathrm{~mL}$ and $1.7 \mathrm{~mL}$ Costar microcentrifuge tubes, Corning, Inc., Corning, New York, USA). No hatched squid remained in the cups across days, so all paralarvae included in the data are from their day of hatching (less than 1 day old).

\section{Metrics}


Methods for measurements of dorsal mantle length (DML) and yolk sac volume (YV) were the same as those reported in Zakroff et al. (2019). In brief, anesthetized paralarvae (around 10 per treatment for the first four days of hatching) were photographed under dissecting microscope (SteREO Discovery.V8, Carl Zeiss AG, Oberkochen, DE) and measured for DML using ImageJ (National Institutes of Health, Rockville, Maryland, USA; Figure 1A). Yolk sac volume was measured by anesthetizing, fixing, and staining paralarvae with oil red $\mathrm{O}$ following Gallager et al. (1986) and processing in ImageJ (Figure 1B) following the methods of Vidal et al. (2002). No premature paralarvae, those with external yolk remaining, were included in either the DML or YV datasets.

Hatching time and success likewise were as described in Zakroff et al. (2019). Hatchling paralarvae were counted and preserved until two days with no paralarvae found in the cup was reached. The egg capsule would then be removed, photographed, and dissected under dissecting scope. The remaining unhatched embryos were counted and categorized by simple visual discrimination of their stage of development (early: stages 1-16, middle: stages 17-26, and late: stages 27-30) adapted from Arnold et al. (1974).

\section{Malformation}

On the largest day of hatching for each cup, a random subsample of around 50 paralarvae were taken and categorized for malformations (sample sizes varied depending on hatching dynamics, but only samples of 20 paralarvae or more were used in the analysis). The subsampled paralarvae were categorized as either Normal: showing no external yolk or malformations, Premature: showing external yolk remaining post-hatch, but no other notable malformations, Eye Bulge: showing an inflation of the membrane around the eyes, or Malformed Head, showing a misshapen, often pointed or oblong head, occasionally also with odd growths or a malformed mantle (Figure 5A).

\section{Statistics}

Statistical analyses were run in a Jupyter Notebook (Project Jupyter) using Python (version 3.5.5, Python Software Foundation). Data were first tested for normality through visual assessment of quantile plots and histograms. Normally distributed data was then processed for group differences of means with multi-factor Type II ANOVAs. ANOVA data are presented 
with calculated effect sizes $\left(\omega^{2}\right)$. A Tukey's HSD posthoc test was used to determine which groups showed statistically significant $(p<0.05)$ differences. All data are reported as means \pm one standard deviation, primarily for easier relation to their visualizations. The yolk volume data was normalized through log transform and then tested as above. It is reported as the back transformed mean and values \pm one standard deviation. Nonparametric water quality data were analyzed with Kruskal-Wallis (KW) tests for difference between treatments. Nonparametric distributional data (hatching time curves, hatching success, and malformation) were analyzed using G-tests and are described for trends with linear regressions (LR), though the statistical power of these regressions is low due to low sample size (data for these metrics is per cup/egg capsule; maximum $n=4$; Table 1). Parametric data are also often presented with a LR trend line, but these are not presented for statistical power; they serve as visual aids of trends.

\section{Assumptions}

Although each trial in this experiment contains eggs from a single, separate mother, since replicates of different mothers were not run at or very near the same time point, the effects of maternity and seasonality cannot be disentangled here. Maternal wet weight is noted in Table 1, but is not included in statistical models, as both the low sample size of mothers and the experimental design did not allow for it to be statistically distinguishable from trial effects.

Number of embryos within an egg capsule was noted in Zakroff et al. (2019) as a potentially impactful continuous variable on the state of hatched paralarvae, particularly for DML. As each cup contained only a single egg capsule in these experiments, any effects particular to the culture cup could not be disentangled from effects of egg capsule (or number of eggs per capsule). Number of eggs per capsule is discussed further in the Results.

On top of trial and cup effects, Zakroff et al. (2019) highlighted different responses to stressors across the days of the squid eggs hatching. While responses do change across hatching days here as well, digging into them is beyond the scope of this manuscript. Further, samples were taken over fewer days of hatching in this dataset (four days compared to six) making the dataset less robust for that type of analysis. Statistical models are presented here with the assumption that effects of hatching date are occurring, but can be ignored in order to investigate the overall impacts of the stressors. 


\section{Results}

\section{Water quality}

Water chemistry, particularly temperature, salinity, and $\mathrm{pH}_{\mathrm{t}}$, was quite stable within and between experiments (Table 1). Seawater alkalinity varied the most in this system, which may have contributed to the variability of the $\mathrm{pCO}_{2}$ equilibrations. Within a treatment, $\mathrm{pH}_{\mathrm{t}}$ and calculated $\mathrm{pCO}_{2}$ were consistent across cups ( $\mathrm{KW}, p>0.05$ for all treatments in all trials). Input gas mixtures were the same across trials, but resultant $\mathrm{pCO}_{2}$ equilibrations were variable between temperature treatments and across trials (Table 1). This variability was most likely due to the flow-through nature of the system and fluctuations in seawater input flow rates, although it may also represent some seawater variability that was note controlled for due to the natural sourcing of water from Vineyard Sound. Equilibrations at higher $\mathrm{CO}_{2}$ concentrations were much more challenging to maintain, resulting in seawater $\mathrm{pCO}_{2}$ values somewhat lower than the input gas concentrations (e.g. $2729.6 \mathrm{ppm}$ for the $20^{\circ} \mathrm{C} \mathrm{X} 3500$ ppm treatment in the Jul 28 trial; Table 1). Despite these variations, data are reported across trials by the input gas concentrations for concision and clarity. However, it should be understood that these three concentrations, 400, 2200, and 3500 ppm, are acting more as a negative control, variable response level, and positive control, respectively, across these experiments rather than a precise representation of response at that equilibrated seawater $\mathrm{CO}_{2}$ concentration.

\section{Dorsal mantle length}

Dorsal mantle length of the paralarvae was impacted by both acidification and warming, but responses varied substantially between trials (Figure 1C). Control treatment $\left(20^{\circ} \mathrm{C} \mathrm{X} 400\right.$ ppm) DML shifted across trials similar to the pattern reported in Zakroff et al. (2019). Notably, this pattern of seasonal DML shift appears unrelated to maternal weight: DML started around the typical parlarvae size of $1.8 \mathrm{~mm}$ (Jun 19, $44.5 \mathrm{~g}$ mother: $1.80 \pm 0.11 \mathrm{~mm}$ ), reached its minimum at the peak of summer (Jul 28, $56.5 \mathrm{~g}$ mother: $1.64 \pm 0.13 \mathrm{~mm}$ ), and then increased again (Sep 14, $62.5 \mathrm{~g}$ mother: $1.74 \pm 0.07 \mathrm{~mm})$. Compiled, the data indicate that while interactions between all factors are significant, the individual factors of trial $\left(\omega^{2}=0.257\right), \mathrm{pCO}_{2}\left(\omega^{2}=0.119\right)$, and temperature $\left(\omega^{2}=0.054\right)$ had the greatest effects on DML (Table 2). Statistical results of significant, relatively strong effects of acidification across trials are driven by the consistent 
efficacy of the $3500 \mathrm{ppm}$ positive control (Figure 1C). Assessments of a response to acidification are therefore focused on results from the $2200 \mathrm{ppm}$ treatment.

Paralarvae from the Jun 19 trial were resistant to both stressors in terms of DML, only showing a notable decrease in size at the $3500 \mathrm{ppm}\left(20^{\circ} \mathrm{C}: 1.63 \pm 0.13 \mathrm{~mm} ; 27^{\circ} \mathrm{C}: 1.64 \pm 0.13\right.$ $\mathrm{mm}$ ) positive control acidification level (Figure 1C, Table 2). The $2200 \mathrm{ppm}$ exposed paralarvae from the $27^{\circ} \mathrm{C}$ treatment $(1.74 \pm 0.11 \mathrm{~mm})$ showed a slight decrease relative to their acidification control $(1.81 \pm 0.14 \mathrm{~mm})$, but were not different from the $2200 \mathrm{ppm}$ from the $20{ }^{\circ} \mathrm{C}$ water bath $(1.78 \pm 0.12 \mathrm{~mm}$; Figure $1 \mathrm{C})$. Interactions between $\mathrm{pCO}_{2}$ and temperature were not significant in this trial (Table 1).

The Jul 28 trial showed a substantial response to temperature in the DML data (decreasing to $1.49 \pm 0.10 \mathrm{~mm}$ at the $27^{\circ} \mathrm{C} \mathrm{X} 400 \mathrm{ppm}$ treatment), but no effect of acidification at the $2200 \mathrm{ppm}$ level (Table 2, Figure 1C). The $3500 \mathrm{ppm}$ positive control resulted in $\mathrm{pCO}_{2}$ having the greatest effect size in this trial $\left(\omega^{2}=0.132\right)$, but temperature was nearly as impactful $\left(\omega^{2}=0.077\right)$, and these stressors appeared to interact slightly $\left(\omega^{2}=0.029\right.$, Table 2$)$.

Decreases in DML were seen in the Sep 14 trial with both acidification at the $2200 \mathrm{ppm}$ treatment $\left(20^{\circ} \mathrm{C}: 1.63 \pm 0.07\right)$ and warming $\left(27^{\circ} \mathrm{C} \mathrm{X} 400\right.$ ppm: $1.53 \pm 0.10 ; 27^{\circ} \mathrm{C} \mathrm{X} 2200$ ppm: $1.49 \pm 0.09 ;$ Figure $1 C)$. Both acidification $\left(\omega^{2}=0.165\right)$ and warming $\left(\omega^{2}=0.252\right)$ had significant impacts on DML, as did their interaction $\left(\omega^{2}=0.090\right)$, which was the largest of all the trials (Table 2).

Notably, warming did not simply transpose the acidification impact downward or exacerbate the slope/severity of acidification effects (Figure 1C). Rather, in trials where warming had a significant effect (Jul 28 and Sep 14), acidification impacts in the warming treatment were decreased (e.g. order of magnitude decrease in slope in Sep $14 ; 20^{\circ} \mathrm{C}$ LR: $-6.97^{*} 10^{-5}, 27^{\circ} \mathrm{C}$ LR: $\left.-8.10^{*} 10^{-6}\right)$. In the compiled data, this results in a shift from a significant decrease with increasing acidification $\left(20^{\circ} \mathrm{C}, \mathrm{LR}\right.$, slope $\left.=-5.75^{*} 10^{-5}, \mathrm{R}^{2}=0.824, p<0.001\right)$ to a slight decrease with increase acidification under warming $\left(27^{\circ} \mathrm{C}, \mathrm{LR}\right.$, slope $=-2.27^{*} 10^{-5}, \mathrm{R}^{2}=0.406, p$ $=0.065$; Figure 1C).

\section{Variance of dorsal mantle length data}

Variance in DML showed broadly similar patterns between the Jun 19 and Sep 14 trials, with variance increasing with acidification at $20^{\circ} \mathrm{C}$ and decreasing with acidification at $27^{\circ} \mathrm{C}$, 
while the Jul 28 trial showed the opposite trends (Figure 1C). As a result, the compiled data show a weak increasing trend with acidification at $20^{\circ} \mathrm{C}\left(\mathrm{LR}\right.$, slope $=1.19^{*} 10^{-6}, \mathrm{R}^{2}=0.445, p=$ $0.057)$ that diminishes to roughly flat line at $27^{\circ} \mathrm{C}\left(\mathrm{LR}\right.$, slope $=4.19^{*} 10^{-7}, \mathrm{R}^{2}=0.025, p=0.682$; Figure 1C). Individual paired t-tests of variance between treatments broadly showed no significant changes in DML variance (two-sample $\mathrm{t}(2), p>0.05$ for most treatment pairings within in each trial), except in the Sep $14,20^{\circ} \mathrm{C}, 400 \times 3500$ test (two-sample t(2) $=-2.96, p=$ 0.042 ), although these results are likely impacted by low sample sizes (Table 1: number of egg capsules per treatment).

Distributions of dorsal mantle length data for each capsule within a treatment were relatively similar in shape, indicating consistency in responses among the egg capsules of a mother's clutch (Sep 14: Figure 2; Jun 19: Figure S1; Jul 28, Figure S2). In the Sep 14 trial, where DML was sensitive to both stressors, egg capsule distribution demonstrated wider spread with decreased peaks under warming (Figure 2). At $20^{\circ} \mathrm{C}$, acidification caused Sep 14 egg capsules to translated to decreased sizes at $2200 \mathrm{ppm}$, but distributions retained the same shape, before flattening, spreading, and become more varied at $3500 \mathrm{ppm}$ (Figure 2).

\section{Yolk sac volume}

Yolk volume responses appear to have been consistently affected (compiled data Type II ANOVA, $p<0.001$ for all factors; Table 2$)$ by both temperature $\left(\omega^{2}=0.045\right)$ and $\mathrm{pCO}_{2}\left(\omega^{2}=\right.$ $0.008)$, but the direction and intensity of those responses shift strongly between trials $\left(\omega^{2}=\right.$ 0.383 , Figure 1C), particularly due to the interaction between trial and warming response $\left(\omega^{2}=\right.$ 0.153). Control treatment hatchling YV followed a similar pattern as DML across trials, decreasing to its minimum in the Jul 28 trial $\left(20^{\circ} \mathrm{C}\right.$ X 400 ppm: Jun 19, $0.030 \mathrm{~mm}^{3}[0.019$ $\left.0.048 \mathrm{~mm}^{3}\right]$; Jul 28, $0.017 \mathrm{~mm}^{3}$ [0.011 $\left.-0.024 \mathrm{~mm}^{3}\right]$; Sep 14, $0.025 \mathrm{~mm}^{3}\left[0.018-0.037 \mathrm{~mm}^{3}\right]$ ).

In the Jun 19 trial, warming appeared to have the most substantial effect $\left(\omega^{2}=0.410\right.$; Table 2) on remaining paralarval yolk reserves, which increased under warming and increased further under combined warming and acidification (Figure 1C). Paralarvae reared at $3500 \mathrm{ppm}$ in the $20^{\circ} \mathrm{C}$ water bath hatched with internal yolk volumes of $0.031 \mathrm{~mm}^{3}\left(0.021-0.046 \mathrm{~mm}^{3}\right)$, similar to the control, while $\mathrm{YV}$ of those in the $27^{\circ} \mathrm{C}$ water bath were $0.240 \mathrm{~mm}^{3}(0.181-0.317$ $\mathrm{mm}^{3}$ ). 
For the Jul 28 trial, while all factors were significant (Table 2), the interaction between warming and acidification had the greatest impact $\left(\omega^{2}=0.045\right)$ on paralarval YV. Similar to the Jun 19 trial, acidification had no notable effect on YV in the $20^{\circ} \mathrm{C}$ water bath and warming increased (though by much less than in Jun 19) remaining YV at the $400 \mathrm{ppm}$ treatment $(0.027$ $\left.\mathrm{mm}^{3}\left[0.014-0.052 \mathrm{~mm}^{3}\right]\right)$. In contrast to Jun 19 , however, $\mathrm{YV}$ in the $27{ }^{\circ} \mathrm{C}$ water bath decreased with increasing acidification in this trial $\left(3500 \mathrm{ppm}, 0.019 \mathrm{~mm}^{3}\left[0.010-0.035 \mathrm{~mm}^{3}\right]\right.$; Figure 1C).

Paralarvae in the Sep 14 clutch showed weak, but significant, overall responses in YV, with temperature having the greatest effect $\left(\omega^{2}=0.109\right.$; Table 2$)$. Unlike the other trials, the Sep 14 paralarvae showed a slight decrease in hatching YV with increasing acidification in the $20^{\circ} \mathrm{C}$ water bath (Figure 1C). Also unique to the Sep 14 trial, warming to $27^{\circ} \mathrm{C}$ resulted in paralarvae hatched with less YV at the $400 \mathrm{ppm}$ treatment $\left(0.016 \mathrm{~mm}^{3}\left[0.010 \mathrm{~mm}^{3}-0.024 \mathrm{~mm}^{3}\right]\right)$. Increasing acidification resulted in slightly increased remnant $\mathrm{YV}$ in the $27{ }^{\circ} \mathrm{C}$ water bath, similar to, but much weaker than, the Jun 19 data (Figure 1C).

Patterns of yolk volume variance were inconsistent across trials and broadly showed no notable trends across acidification (two-sample $\mathrm{t}(2), p>0.05$ for most treatment pairings within in each trial). A significant decrease in yolk volume variance was seen in the $27^{\circ} \mathrm{C}$ water bath of the Jun 19 paralarvae between both the $400 \mathrm{ppm}\left(1.044 \pm 0.079 \mathrm{~mm}^{6}\right.$; two-sample $\mathrm{t}(2)=13.30, p$ $<0.001)$ and $2200 \mathrm{ppm}\left(0.935 \pm 0.061 \mathrm{~mm}^{6}\right.$; two-sample $\left.\mathrm{t}(2)=13.92, p=0.005\right)$ treatments and the 3500 ppm sample $\left(0.083 \pm 0.007 \mathrm{~mm}^{6}\right)$.

\section{Comparing DML \& $Y V$}

In order to investigate clutch-specific patterns of physiological response to both acidification and warming stress, YV was plotted against DML for each egg capsule of each treatment (Figure 1D). The $27^{\circ} \mathrm{C}$ treatment of the Jun 19 showed the strongest trend (LR, slope $=-8.60, \mathrm{R}^{2}=0.865, p=0.002$; Figure $\left.1 \mathrm{D}\right)$, with warming and acidification having resulted in smaller paralarvae with less consumed yolk before hatching. The Jul 28 eggs, conversely, showed a weak trend of smaller paralarvae hatching with more yolk consumed under the same conditions. The Sep 14 clutch demonstrates a much weaker trend under both acidification and warming, but of a similar response type to the Jun 19 clutch. This trial also differs by having the only positive slope of the $20^{\circ} \mathrm{C}$ exposures $(\mathrm{LR}$, slope $=0.020$ ), with paralarvae having hatched smaller and with less yolk under increased acidification (Figure 1D). 


\section{Egg number}

Statistical models were run with number of eggs per capsule as an independent continuous covariate, but did not appear to have a significant effect on DML or YV in these experiments (multi-factor Type II ANOVA, $p>0.05$ for all trials and in combined data) and so are not presented here.

\section{Hatching Time}

Increased temperature increased the rate of embryonic development, resulting in $27{ }^{\circ} \mathrm{C}$ egg capsules consistently hatching sooner (around 9 days) than their $20{ }^{\circ} \mathrm{C}$ counterparts (around 14-15 days) in all trials (Figure 3A). While time to hatching increased for the $20{ }^{\circ} \mathrm{C}$ treatments across the breeding season, as was seen in Zakroff et al. 2019, this seasonal increase in hatching time disappears in the $27^{\circ} \mathrm{C}$ treatments (see y-intercepts in Figure 3B). Increasing acidification broadly delayed hatching by around 1.5 days, but these impacts were somewhat dampened by warming, although responses to combined stressors varied across trials (see slopes in Figure 3B).

In the Jun 19 eggs, time to $50 \%$ hatching was delayed in the $20^{\circ} \mathrm{C}$ treatment from 14.09 \pm 0.40 days at $400 \mathrm{ppm}$ to $14.79 \pm 0.10$ days at $2200 \mathrm{ppm}$ and $15.13 \pm 0.32$ days at $3500 \mathrm{ppm}$ (Figure 3B). Hatching distributions were significantly different between the $400 \mathrm{ppm}$ and both increased acidification treatments $(\mathrm{G}(7), p<<0.001$ for both pairs) at this temperature, but the 2200 and 3500 ppm curves were not statistically distinct $(\mathrm{G}(6)=4.699, \mathrm{p}=0.583)$. In the $27^{\circ} \mathrm{C}$ treatment, time to $50 \%$ hatching was delayed from $9.67 \pm 0.08$ days at $400 \mathrm{ppm}$ and $9.83 \pm 0.05$ days at $2200 \mathrm{ppm}$ to $11.01 \pm 0.32$ days at $3500 \mathrm{ppm} \mathrm{CO}_{2}$. At this temperature, all hatching curves were different from each other $\left(\mathrm{G}(7), p<<0.001\right.$ for all $\mathrm{pCO}_{2}$ treatment pairs), but the differences between 400 and 2200 ppm were two orders of magnitude lower (G statistic of around 50 compared to around 1000) than pairings with the $3500 \mathrm{ppm}$ treatment.

Delays in hatching occurred more consistently and progressively with increasing acidification in the Jul 28 trial (Figure 3). Within each temperature treatment, each hatching distribution at each $\mathrm{pCO}_{2}$ treatment was significantly different from each other $\left(20^{\circ} \mathrm{C}, \mathrm{G}(6), \mathrm{p}<<\right.$ $0.001 \& 27^{\circ} \mathrm{C}, \mathrm{G}(11), p<<0.001$ for all $\mathrm{pCO}_{2}$ treatment pairs). Time to $50 \%$ hatching increased at $20^{\circ} \mathrm{C}$ from $15.04 \pm 0.49$ days at $400 \mathrm{ppm}$ to $15.92 \pm 0.27$ days at $2200 \mathrm{ppm}$ and $16.54 \pm 0.36$ 
days at $3500 \mathrm{ppm}$. At $27^{\circ} \mathrm{C}, 50 \%$ hatching was delayed from $9.81 \pm 0.59$ days, to $10.52 \pm 0.70$ days, then to $11.22 \pm 1.62$ days with increasing acidification (Figure 3B).

While hatching was clearly delayed in the $20^{\circ} \mathrm{C}$ water bath of the Sep 14 trial, acidification responses were strongly dampened at $27^{\circ} \mathrm{C}$ (Figure 3). Distributions of cumulative percent hatching were statistically distinct in both the $20^{\circ} \mathrm{C}\left(\mathrm{G}(6), p<<0.001\right.$ for all $\mathrm{pCO}_{2}$ treatment pairs) and $27^{\circ} \mathrm{C}\left(\mathrm{G}(11), p<<0.001\right.$ for all $\mathrm{pCO}_{2}$ treatment pairs) water baths, but the differences (as assessed by $\mathrm{G}$ statistics and $\mathrm{p}$ values) are an order of magnitude higher in the 20 ${ }^{\circ} \mathrm{C}$ samples. Time to $50 \%$ hatching had the greatest delay in the $20{ }^{\circ} \mathrm{C}$ samples of the Sep 14 trial, increasing from $15.83 \pm 0.06$ days at $400 \mathrm{ppm}$ to $17.87 \pm 0.452$ at $3500 \mathrm{ppm}$ (Figure 3B).

Contrastingly, this trial also had the smallest delay in its $27^{\circ} \mathrm{C}$ samples, increasing from $9.54 \pm$ 0.18 days to $10.04 \pm 0.14$ days.

\section{Hatching Success}

Hatching success decreased both with acidification and warming, with increased acidification typically resulting more late stage losses, while warming resulted in more early to middle stage losses (Figure 4). Response patterns in hatching success were unique in each trial, as with previous metrics.

In the Jul 19 trial, hatching was quite high in the $400(98.9 \pm 1.0 \%)$ and $2200 \mathrm{ppm}(92.7$ $\pm 3.9 \%)$ treatments of the $20^{\circ} \mathrm{C}$ and the $400 \mathrm{ppm}$ at $27^{\circ} \mathrm{C}(96.1 \pm 2.9 \%)$. Distributions of staged failed embryos and hatched paralarvae were significantly different for all $\mathrm{pCO}_{2}$ treatment combinations within each temperature ( $\mathrm{G}(3), p<0.001$ for all $\mathrm{pCO}_{2}$ treatment pairs) and for all $\mathrm{pCO}_{2}$ comparisons across temperatures $(\mathrm{G}(3), p<0.001$ for comparison of 2200 and $3500 \mathrm{ppm}$ across temperatures) except at $400 \mathrm{ppm}(\mathrm{G}(3)=5.313, p=0.150$; Table S1). The $2200 \mathrm{ppm}$ at 27 ${ }^{\circ} \mathrm{C}$ sampled had a single egg capsule completely fail at the middle stages, which drove down over hatching success for the treatment $(59.2 \pm 42.2 \%)$. The $3500 \mathrm{ppm}$ treatments at both temperatures $\left(20^{\circ} \mathrm{C}: 73.2 \pm 10.3 \% ; 27^{\circ} \mathrm{C}: 84.1 \pm 6.6 \%\right)$ had decreased hatching due to losses at late stages.

Egg capsules of the Jul 28 trial showed high hatching success across acidification treatments at $20^{\circ} \mathrm{C}$ (all above $90 \%$; Table S1), but had substantive decreases in the 400 (34.0 \pm $31.9 \%)$ and $3500 \mathrm{ppm}(54.7 \pm 36.3 \%)$ treatments at $27^{\circ} \mathrm{C}$. Embryos halted development in multiple egg capsules of the $400 \mathrm{ppm}$ treatment at all stages, but mostly early, while late stage 
losses drove the decrease in hatching success at $3500 \mathrm{ppm}$ (Table S1). Hatching success distributions were all distinct for all treatments within this trial $(\mathrm{G}(3), p<0.001$ for almost all $\mathrm{pCO}_{2}$ and temperature treatment pairs; Table S1), though the 400 and $3500 \mathrm{ppm}$ treatments at 20 ${ }^{\circ} \mathrm{C}$ were nearly the same due to the presence of slight late stage losses $(\mathrm{G}(3)=10.77, p=0.013)$.

Hatching success was highest in the $400(94.0 \pm 5.0 \%)$ and $2200 \mathrm{ppm}(93.8 \pm 2.8 \%)$ treatments at $27^{\circ} \mathrm{C}$ in the Sep 14 data (Table S1), which showed very similar distributions with only slight early stage losses $(\mathrm{G}(3)=3.524, p=0.318)$. All other treatment combinations showed significant differences in hatching success distribution within this trial $(\mathrm{G}(3), p<0.001$; Table $\mathrm{S} 1)$. The $3500 \mathrm{ppm}$ treatment at $27^{\circ} \mathrm{C}$ had low hatching success $(56.8 \pm 40.7 \%)$ driven by near complete early stage loss in a single capsule, as well as slight early stage losses in the other capsules. The $400 \mathrm{ppm}$ treatment at $20^{\circ} \mathrm{C}$ had relatively high hatching success $(85.1 \pm 8.3 \%)$, although lower compared to the $27{ }^{\circ} \mathrm{C}$ due to high early stage losses. Increased acidification at 20 ${ }^{\circ} \mathrm{C}$ had much higher decreases in hatching success, however, due to large late stage losses in both the $2200(57.8 \pm 41.0 \%)$ and $3500 \mathrm{ppm}(74.2 \pm 15.0 \%)$ treatments.

\section{Malformation}

The patterns of malformation were relatively consistent across trials, with increasing acidification producing greater proportions of premature and eye bulge paralarvae, while warming dampened the acidification impacts slightly and increased the proportion of malformed head paralarvae (Figure 5B). In all trials, proportionally less premature paralarvae were seen in the $3500 \mathrm{ppm}$ treatment at $27^{\circ} \mathrm{C}$ than at $20^{\circ} \mathrm{C}$ (Table S2). Distributions of categorized paralarvae for each treatment combination compared across trials, showed significant shifts as a result of trial in the $20^{\circ} \mathrm{C}$ X $2200 \mathrm{ppm}$ treatment and all $27^{\circ} \mathrm{C}$ treatments $(\mathrm{G}(6), p<0.001$ for listed treatments).

Paralarvae from the Jun 19 trial generally demonstrated the pattern described above, but with particularly notable increases in malformed head paralarvae in all acidification levels at 27 ${ }^{\circ} \mathrm{C}$ (Figure 5; Table S2). All distributions in all within temperature and within $\mathrm{pCO}_{2}$ pairings in this trial significantly differed from each other (G(3), $p<0.01$; Table $\mathrm{S} 2)$.

In the Jul 28 trial, paralarvae showed broadly similar patterns of malformation across temperatures, with acidification impacts being slightly more prominent in the $20^{\circ} \mathrm{C}$ and temperature impacts being minimal (in part possibly driven by sample size shifts between 
temperatures; Table S2). Although all within temperature comparisons of malformation distributions between $\mathrm{pCO}_{2}$ treatments were significant, those at $27{ }^{\circ} \mathrm{C}$ were less different than those at $20^{\circ} \mathrm{C}(\mathrm{G}(3), p \leq 0.001$; see exponents in Table S2). Notably, distributions of malformations between $\mathrm{pCO} 2$ treatments across temperatures were either comparatively weakly, in the case of $400 \mathrm{ppm}(\mathrm{G}(3)=11.57, p=0.009)$, or not significantly different, for $2200(\mathrm{G}(3)=$ $4.485, p=0.214)$ and $3500 \mathrm{ppm}(\mathrm{G}(3)=0.338, p=0.953)$, in this trial.

The Sep 14 paralarvae also demonstrated more prominent acidification impacts at $20^{\circ} \mathrm{C}$ that were slightly dampened with warming, which only showed a slight, but weak, increase in malformed head proportions (Figure 5). Distributions of malformations were significantly different between all $\mathrm{pCO}_{2}$ treatment pairs within temperatures ( $\mathrm{G}(3), p<0.001$; Table S2) owing to increasing premature and eye bulge proportions with increasing acidification. Differences across temperatures, driven by the dampening and shifts described with warming above, were significant in the $400 \mathrm{ppm}(\mathrm{G}(3)=16.32, p<0.001)$ treatment and neared significance in the $2200(\mathrm{G}(3)=6.622, p=0.085)$ and $3500 \mathrm{ppm}(\mathrm{G}(3)=6.829, p=0.078)$ treatments.

\section{Discussion}

These experiments demonstrated clutch-dependent sensitivity to high levels of combined acidification and warming stress in the egg capsules of $D$. pealeii across the 2016 breeding season. Embryos appear to be capable of developing normally, at least in terms of size, yolk consumption, and survival up to at least $2200 \mathrm{ppm} \mathrm{CO}_{2}$, a value which is not predicted in open ocean, no reduction of emissions scenarios until at least the year 2300 (Caldeira and Wickett 2003). The consistent hatching delay, as well as the increased proportion of late stage loss and premature paralarvae observed, suggest that acidification may cause metabolic suppression, particularly late in development, as was described for L. vulgaris embryos under warming (Pimentel et al. 2012; Rosa et al. 2012). Metabolic suppression was long suggested as an expected impact of $\mathrm{OA}$ on squid because squid hemocyanins are very sensitive to $\mathrm{pH}$, squid operate at the very peak of blood oxygen utilization, and the resultant Bohr shift would starve the animal of oxygen (Pörtner 1990, 1994; Fabry et al. 2008; Seibel 2016). While metabolic suppression was observed in jumbo squid, Dosidicus gigas, exposed to $1000 \mathrm{ppm} \mathrm{CO}_{2}$, more recent studies have demonstrated no metabolic impacts to adults of bigfin reef squid, 
Sepioteuthis lessoniana, and pygmy reef squid, Idiosepius pygmaeus, as well as adults and juvenile D. gigas, and D. pealeii at equal or greater levels of acidification (Rosa and Seibel 2008; Hu et al. 2014; Birk et al. 2018; Spady et al. 2019). It is possible that metabolic sensitivity to acidification is life stage-dependent in cephalopods with juveniles and adults having the robust physiological machinery needed to manage under OA stress, while the gears of development may be slowed in embryos. Of particular interest, then, is the metabolic scope of the paralarvae under acidification, which are thought to be quite sensitive based on aquaculture studies $(\mathrm{pH}$ range of 8.1-8.4 for loliginid paralarvae), and the transition from paralarvae to juvenile in squid (Vidal et al. 2002b).

Observed impacts of acidification to the squid suggest systems of $\mathrm{pH}$ and ionic/osmotic balance may be strained, particularly under the severe dosage of $3500 \mathrm{ppm}$. Zakroff et al. (2019) discussed potential mechanisms of acidification impact to DML and YV, in the context of a limited energy store and energy budget, suggesting that reductions in growth and yolk volume under hatching are a potential product of upregulation and increased activity of energeticallycostly proton secreting transporters in ion-transport epithelia (Hu et al. 2010, 2013). The increased proportion of paralarvae showing inflation of the membrane around the eyes under increased OA further suggests a breakdown in osmoregulatory controls, particularly given the prevalence of ionocytes in the epidermis of cephalopod embryos (Hu et al. 2011b). Though it is also plausible that this inflation is related to poorly known osmotic mechanisms that cause the swelling of the egg capsule during development (Hu and Tseng 2017).

In sensitive clutches, warming strongly impacted development time, DML and YV, hatching success, and malformations, likely through an increase in metabolic and developmental rates. Warming of $+2{ }^{\circ} \mathrm{C}$ is a standard experimental choice given predicted scenarios under no emission reductions (Pachauri and Meyer 2014). $27^{\circ} \mathrm{C}\left(+2{ }^{\circ} \mathrm{C}\right.$ above peak for Vineyard Sound) is within the habitat window reported for $D$. pealeii juveniles and adults, but well above the 23 ${ }^{\circ} \mathrm{C}$ maximum reported for egg laying habitats (Jacobson 2005). Cephalopod eggs typically demonstrate curves wherein hatching success is quite high $(>80 \%)$ within the preferred window and then drops off rapidly and precipitously above and below certain temperature thresholds (Cinti et al. 2004; Staaf et al. 2011; Zeidberg et al. 2011). This threshold was reported to be between $22-25^{\circ} \mathrm{C}$ in $D$. opalescens, but appears to be higher for D. pealeii (Zeidberg et al. 2011). Loss of embryos under warming was primarily in early- and mid-stages of development, 
suggesting, despite acclimation, a clear and immediate impact to physiology that could easily push embryos past their limits. Disruption of the developmental machinery by warming likely also explains the increased proportion of paralarvae with malformed heads and odd growths, as has been described in L. vulgaris (Rosa et al. 2012).

Contrary to some of the observations of temperature and acidification compounding stress effects in L. vulgaris embryos and paralarvae, these stressors appear to act antagonistically for most of the factors we measured in the early life stages of D. pealeii (Rosa et al. 2014a). In part, this antagonism may be due to the much stronger effect size of warming in sensitive clutches. In the DML data, for example, warming may have driven embryos to their size floor, the minimum size viable for a paralarvae to hatch, and therefore no further decreases due to acidification could be observed. The two stressors counteract most clearly in hatching time, where warming increases oxygen consumption and developmental rate, while acidification causes developmental delay and potentially metabolic suppression (Pimentel et al. 2012; Rosa et al. 2014a; Navarro et al. 2016; Zakroff et al. 2019). In hatching success and malformation, the dampening of acidification impacts is likely driven by a reduction in acidification exposure time as a result of the drastic decrease in time to hatching. The data compiled here suggests that warming impacts $D$. pealeii eggs early in development with disruptions, like malformed bodies, potentially propagating to hatching if development doesn't cease altogether. OA, conversely, appears to be a slow burn across development, compounding the buildup of $\mathrm{CO}_{2}$ and acidification that would naturally occur due to respiration and thereby causing greater impacts to late stage embryos (Gutowska and Melzner 2009; Long et al. 2016).

Each clutch of eggs (each trial) demonstrated a different set of responses to acidification and warming across most metrics, particularly DML and YV. As in Zakroff et al. (2019), the comparison of these metrics provides potential insight into the range of physiological coping responses available to D. pealeii embryos under multiple stressors (Figure 1D). In the Jun 19 trial, eggs under warming and acidification were smaller in size with less consumed yolk, suggesting an overall metabolic suppression that resulted in a relatively resistant clutch. The Jul 28 trial showed the reverse, with more yolk consumed in smaller paralarvae of the combined acidification and warming treatment, suggesting warming outpaced acidification, taxing embryos to consume more yolk. The Sep 14 clutch showed larger paralarvae with slightly more yolk in the control condition, indicating both acidification and warming taxed the energy budgets of 
these developing embryos. Unfortunately, while it is possible to culture myopsid paralarvae in aquaria (Vidal et al. 2002b), it is a very challenging proposition for D. pealeii that we tried, but could not accomplish here. Thus, a question that remains is: given these differential responses to the same stressors between clutches, which strategy would produce the most viable paralarvae in a stressful ocean?

There were two aspects of clutch variability highlighted in these experiments, the first of which is variability between clutches/mothers (which cannot be disentangled from seasonality here). Parental conditioning has been shown to impact sensitivities conferred to offspring in fishes and corals (Miller et al. 2012; Putnam and Gates 2015; Schunter et al. 2016, 2018). Murray et al. described seasonal pH conditioning of parents in a coastal fish, Menidia menidia, which resulted in differential pH sensitivity in offspring (Murray et al. 2014). In cephalopods, embryos from winter and summer cohorts of L. vulgaris were shown to respond very differently to acidification and warming stress, with summer cohorts being more sensitive (Rosa et al. 2014a). Scientists, staff, and fisherman that work with D. pealeii at the various scientific institutions in Woods Hole, MA, USA anecdotally acknowledge the presence of cohorts within the breeding season, or at least a succession of size classes, but this shift has only roughly been described in the literature as a transition between an early two-year old cohort and the new oneyear old cohort (Arnold et al. 1974; Mesnil 1977). In Zakroff et al. (2019) sensitivity to acidification started strong and decreased as the season went on, while here, the earliest trial was the most resistant and the latest the most sensitive. This appears to indicate some form of change in parental conferred sensitivity across the 2013 and 2016 seasons from the early summer squid to the early autumn squid, although in opposite directions between these years, which may support the idea of shifting cohorts within the breeding season and suggests that it is parentage rather than seasonality that is driving offspring sensitivity.

The second form of egg clutch variability examined here is variability between the egg capsules of a single mother's clutch. Even among egg capsules of a single female, squid parentage is a complex proposition since mating can occur with, and sperm can be stored from, multiple males (Buresch et al. 2006). Stress responses and statolith elemental composition have been observed to vary between egg capsules in D. opalescens (Navarro et al. 2014, 2016). Ikeda et al. noted variability within the paralarvae from a single $S$. lessoniana mother, indicating a range of mantle lengths correlated with statolith size and hatching time (1999). The results here 
suggest that, for DML at least, each egg capsule results in a hatch of paralarvae with sizes approximating a normal distribution, but each egg capsule from an individual mother has about the same distribution (Figure 2). Under stress however, these distributions shift. Relatively light stress appears to simply shift the distributions, while heavier stressors cause flattening and spreading of these curves (Figure 2). It has been theorized that size variation among offspring acts as a kind of adaptation to unpredictable environments, with selection pressures (in this case increased environmental stress) acting upon both the mean and variance of an offspring distribution (Marshall et al. 2008).

In cephalopods, paralarval size is, on a taxonomic level, known to correlate to egg size (Laptikhovsky et al. 2013). Squid do not retain lipid reserves, so maternal investment in reproduction is primarily driven by the allocation of resources between somatic vs. reproductive growth (Pecl and Moltschaniwskyj 2006). Energy for egg production is captured through recent feeding, so while successive clutches of eggs may degrade in quality as the state of the mother degrades, maternal input within a clutch may, as appears to be supported here by DML evidence, be relatively consistent across egg capsules (Steer et al. 2004). It is plausible then that the breakdown of similarities between egg capsules under stress could owe to differences in genetic background due to paternity, though this is purely speculative without much more robust experimentation.

This study demonstrated that D. pealeii embryos and paralarvae reared under severe, chronic acidification and warming could show a range of responses from sensitive to resistant. These responses are driven by between clutch differences, which are likely representations of parentage, but may also be influenced by seasonality. Responses are also variable given the complexity of interacting and antagonistic physiological processes influenced by warming and acidification in this system. These experiments were limited in a number of key ways. As an in lab experiment, factors of flow, egg capsule density, and variability that occur in the natural system are not represented here. Variability of $\mathrm{pH}$ in natural systems is thought to decrease impacts in some organisms by reducing exposure time, which appears to be an important factor in acidification's impact on D. pealeii eggs (Shaw et al. 2013). While a growing body of literature is beginning to suggest squid, at least embryos and adults (there is still a great deal left to understand with respect to paralarvae), may be fairly robust in the face of ocean acidification, these responses may be taxon-, population-, or region-specific making it difficult to generalize 
(Kroeker et al. 2017; Birk et al. 2018; Spady et al. 2019). Warming, however, clearly places limits on D. pealeii embryonic development, but squid have the advantage of mobility in coping with that (Doubleday et al. 2016). A fecund, plastic, year class species, such as Doryteuthis pealeii, appears well suited to rapid adaptability under rapid global ocean change. It is important, therefore, to continue to describe the signs and understand the mechanisms of that adaptability, and to investigate its limits, in order to inform how we design experiments to diagnose sensitivity and adaptability in other marine taxa.

\author{
Abbreviations \\ DML - Dorsal mantle length \\ ESL - Environmental systems laboratory \\ KW - Kruskal-Wallis test \\ LR - Linear regression \\ MBL - Marine biological laboratories \\ OA - Ocean acidification \\ YV - Yolk sac volume
}

\title{
Funding
}

This research was supported by National Science Foundation Grant No. 1220034 to TAM and the National Science Foundation Graduate Research Fellowship under Grant No. 1122374 to CZ.

\section{Acknowledgments}

We thank D. Remsen, the MBL Marine Resources Center staff, and MBL Gemma crew for their help acquiring squid. R. Galat and WHOI facilities staff aided with set up, maintenance, and system support at the ESL. D. McCorkle provided guidance and insight on the acidification system and water quality monitoring, as well as the spectrophotometric $\mathrm{pH}$ system, with methods from M. White. Assistance and advice from A. Schlunk and L. Fitzgerald during these experiments was incredibly beneficial. 


\section{Bioethics}

All applicable international, national, and/or institutional guidelines for the care and use of cephalopods were adhered to. 


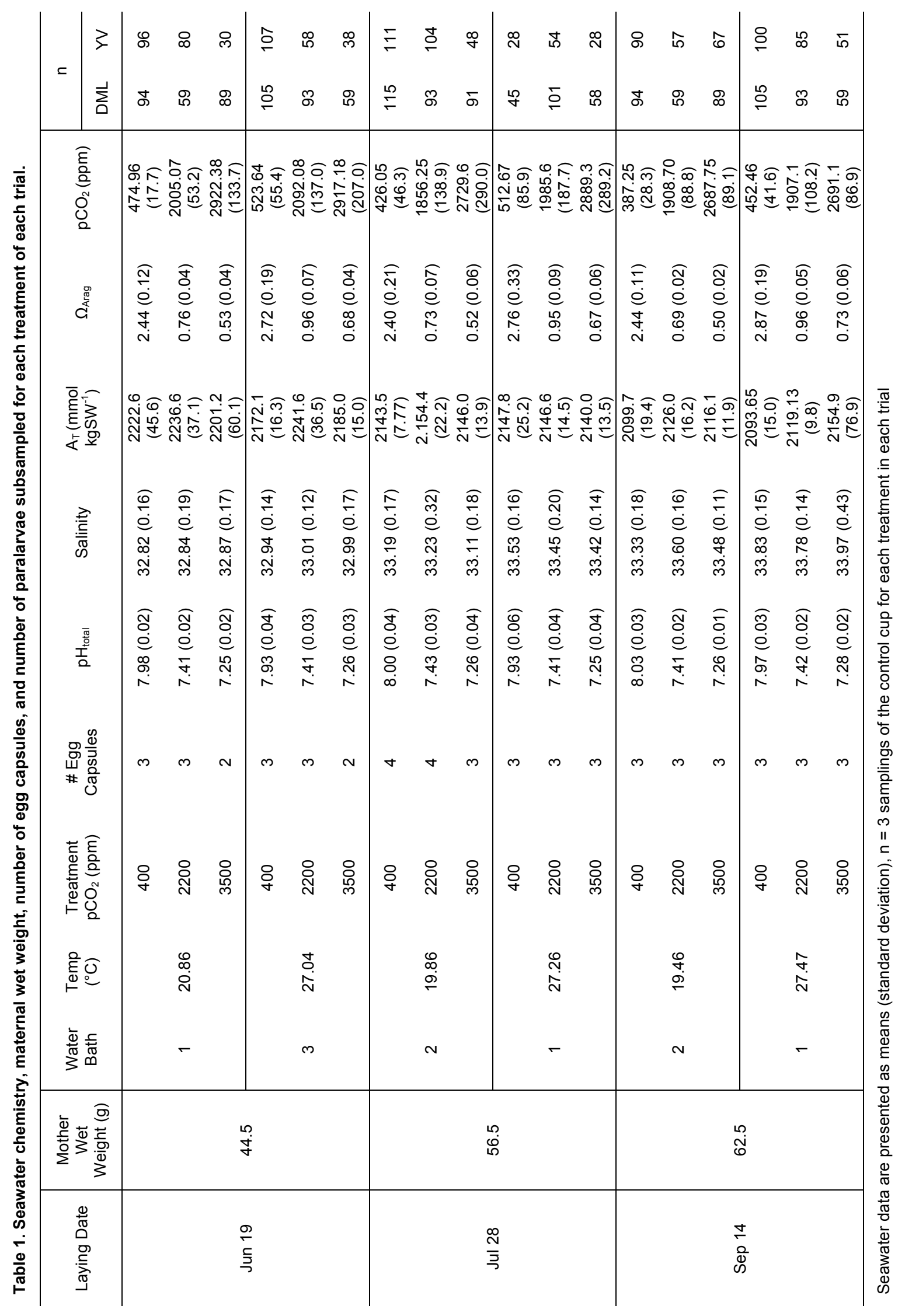


Table 2. Three-way Type II ANOVAs for dorsal mantle length and (log-transformed) yolk volume data, for each trial and compiled across trials. Significant $p$ values $(\alpha=0.05)$ in bold. ' $<<$ ' indicates a $p$-value with a negative exponent of 50 or greater.

\begin{tabular}{|c|c|c|c|c|c|c|c|c|c|c|}
\hline \multirow[b]{2}{*}{ Source } & \multicolumn{5}{|c|}{ Mantle Length } & \multicolumn{5}{|c|}{ Yolk Sac Volume } \\
\hline & SS & $\mathrm{df}$ & $F$ & $P$ & $\omega^{2}$ & SS & $\mathrm{df}$ & $F$ & $P$ & $\omega^{2}$ \\
\hline \multicolumn{11}{|l|}{ Jun 19} \\
\hline Temp & 0.011 & 1 & 0.728 & 0.394 & -0.000 & 165.9 & 1 & 326.3 & $<<0.001$ & 0.410 \\
\hline $\mathrm{pCO}_{2}$ & 2.146 & 2 & 69.37 & $<0.001$ & 0.217 & 18.52 & 2 & 18.21 & $<0.001$ & 0.043 \\
\hline Temp : $\mathrm{pCO}_{2}$ & 0.064 & 2 & 2.059 & 0.129 & 0.003 & 13.86 & 2 & 13.63 & $<0.001$ & 0.032 \\
\hline Residual & 7.516 & 486 & & & & 204.9 & 403 & & & \\
\hline \multicolumn{11}{|l|}{ Jul 28} \\
\hline Temp & 0.887 & 1 & 52.11 & $<0.001$ & 0.077 & 1.780 & 1 & 9.091 & 0.002 & 0.020 \\
\hline $\mathrm{pCO}_{2}$ & 1.514 & 2 & 44.46 & $<0.001$ & 0.132 & 1.358 & 2 & 3.466 & 0.032 & 0.012 \\
\hline Temp : $\mathrm{pCO}_{2}$ & 0.362 & 2 & 10.62 & $<0.001$ & 0.029 & 3.942 & 2 & 10.06 & $<0.001$ & 0.045 \\
\hline Residual & 8.460 & 497 & & & & 71.87 & 367 & & & \\
\hline \multicolumn{11}{|l|}{ Sep 14} \\
\hline Temp & 2.203 & 1 & 256.4 & $<0.001$ & 0.252 & 9.148 & 1 & 59.41 & $<0.001$ & 0.109 \\
\hline $\mathrm{pCO}_{2}$ & 1.453 & 2 & 84.52 & $<0.001$ & 0.165 & 1.928 & 2 & 6.273 & 0.002 & 0.020 \\
\hline Temp : $\mathrm{pCO}_{2}$ & 0.800 & 2 & 46.51 & $<0.001$ & 0.090 & 3.341 & 2 & 10.87 & $<0.001$ & 0.037 \\
\hline Residual & 4.237 & 493 & & & & 68.25 & 444 & & & \\
\hline \multicolumn{11}{|l|}{ Compiled } \\
\hline Trial & 10.32 & 2 & 376.6 & $<<0.001$ & 0.257 & 352.2 & 2 & 619.5 & $<<0.001$ & 0.383 \\
\hline Temperature & 2.148 & 1 & 156.9 & $<0.001$ & 0.054 & 41.59 & 1 & 146.3 & $<0.001$ & 0.045 \\
\hline $\mathrm{pCO}_{2}$ & 4.768 & 2 & 174.1 & $<<0.001$ & 0.119 & 7.062 & 2 & 12.42 & $<0.001$ & 0.008 \\
\hline Trial : Temp & 1.056 & 2 & 38.52 & $<0.001$ & 0.026 & 140.2 & 2 & 246.7 & $<<0.001$ & 0.153 \\
\hline Trial : $\mathrm{pCO}_{2}$ & 0.408 & 4 & 7.443 & $<0.001$ & 0.010 & 11.93 & 4 & 10.50 & $<0.001$ & 0.013 \\
\hline Temp : $\mathrm{pCO}_{2}$ & 0.782 & 2 & 28.54 & $<0.001$ & 0.019 & 6.433 & 2 & 11.32 & $<0.001$ & 0.007 \\
\hline $\begin{array}{l}\text { Trial : Temp } \\
: \mathrm{pCO}_{2}\end{array}$ & 0.443 & 4 & 8.093 & $<0.001$ & 0.011 & 14.71 & 4 & 12.94 & $<0.001$ & 0.016 \\
\hline Residual & 20.21 & 147 & & & & 345.1 & 121 & & & \\
\hline
\end{tabular}


Figure 1. Morphometrics measured on Doryteuthis pealeii paralarva through microscope imagery. (A) Dorsal mantle length (DML; the superimposed yellow line) of an anesthetized paralarvae. (B) Squid paralarvae were fixed and stained with oil red $\mathrm{O}$ for measurement of yolk sac volume. The length and width of both the anterior yolk sac (AYS), which was modeled as either a cone or cylinder (cone, in this case), and the posterior yolk sac (PYS), which was modeled as an ellipsoid were measured (superimposed yellow lines). Each image has a unique 1 $\mathrm{mm}$ white scale bar at bottom left. (C) Dorsal mantle length, its variance, and yolk sac volume data from three experiments rearing squid eggs under acidification (in parts per million $\mathrm{CO}_{2}$; $\mathrm{x}-$ axis) and warming (color; blue/dark $=20^{\circ} \mathrm{C}$, red/light $=27^{\circ} \mathrm{C}$ ). Yolk data was transformed to logarithmic scale for statistical analyses and has been back transformed for the depiction of trial data. The scale of the y-axis in the yolk data changes from the Jun 19 and Jul 28 trials because the Jun 19 paralarvae showed substantially more yolk than the Jul 28 and Sep 14 trials. Symbols represent trial, demarcated by dates eggs were laid (titles). The compiled plots show data from all trials normalized by subtracting the average value for a trial from its data; relative differences in yolk data have not been back transformed from the logarithmic scale. Symbols depict means with error bars of one standard deviation. Letters indicate statistical groups across acidification levels within a temperature treatment from a Tukey's HSD. Asterisks indicate statistically significant differences between temperature treatments at the same acidification level. Regression lines are presented primarily as an aid to visualizing trends in the data and are not intended to indicate statistical power. (D) Response of individual egg capsules within a clutch to acidification and warming as indicated by comparing yolk volume (y-axis; note again scale shift from Jun 19 to Jul 28) with DML (x-axis). Shape indicates trial (also denoted by title). Color and symbols within a plot denote temperature treatments: blue/dark with circle/square/downward triangle $=20^{\circ} \mathrm{C}$; red/light with hexagon/diamond/upward triangle $=27^{\circ} \mathrm{C}$ ) while shade indicates acidification treatment $($ darker $=$ more acidic). Symbols represent the means for a single egg capsule (one cup within the experimental system). No error bars are depicted for visual clarity. Trend lines are regressions of each temperature treatment, but are only presented as visual aides 

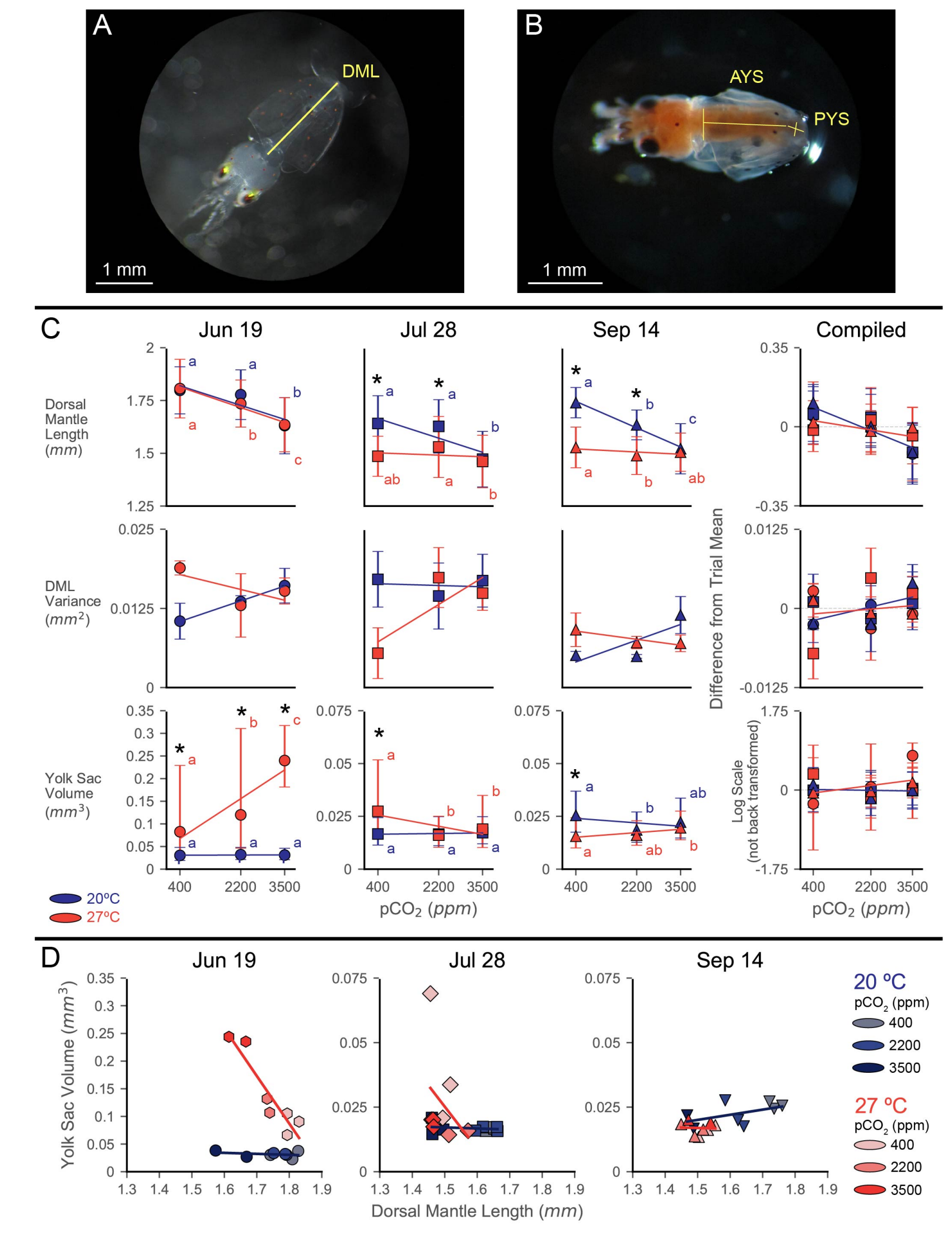


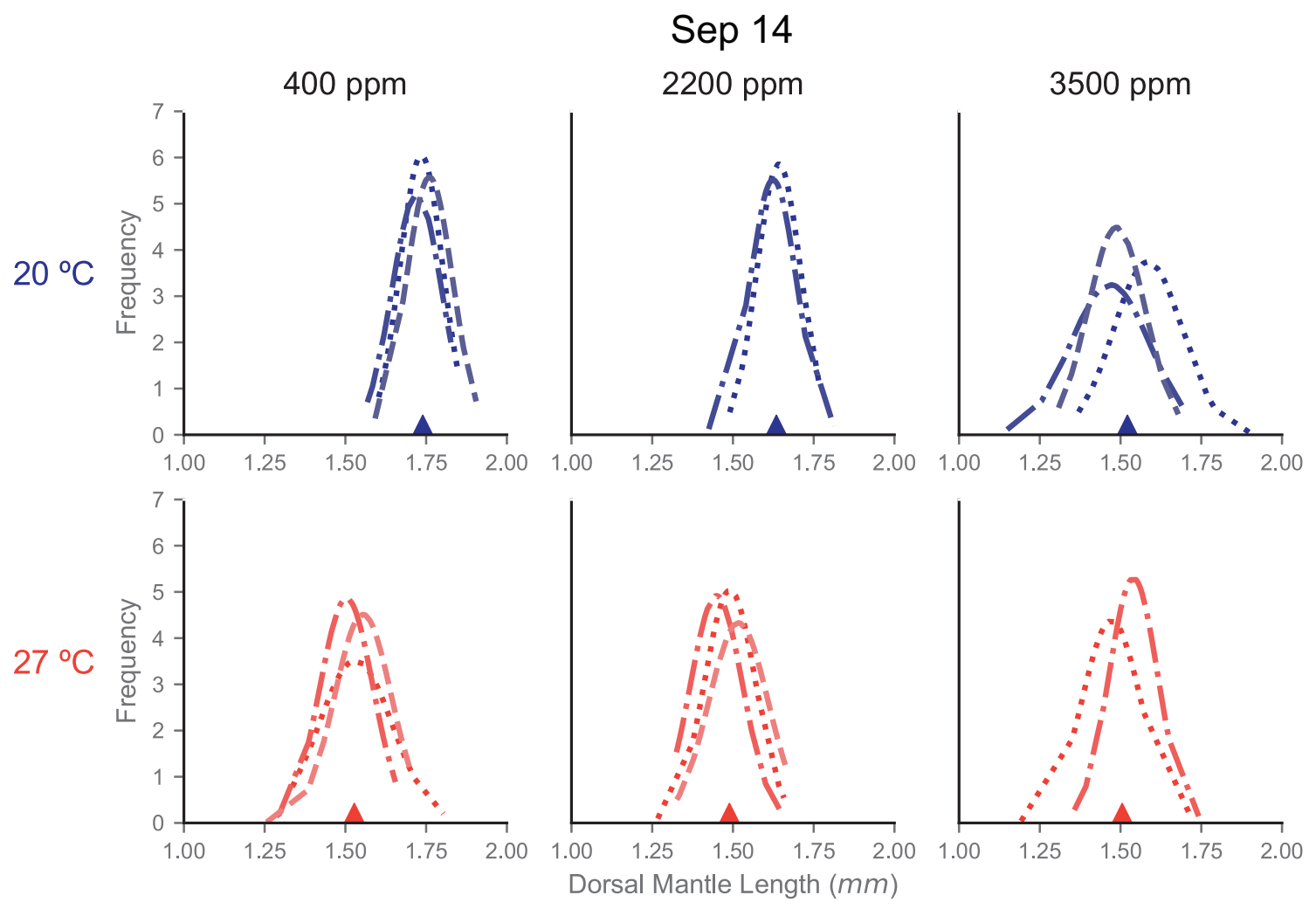

Figure 2. Fitted normal distributions of dorsal mantle length frequency histograms from individual egg capsules in each treatment of the September 14 trial. Histograms are not shown for clarity of curves, but DML data were segmented in $0.05 \mathrm{~mm}$ bins. Each plot is a treatment combination, with column determining acidification (titles) and row determining temperature treatment (also differentiated by color: top/blue $=20^{\circ} \mathrm{C}$, bottom $/ \mathrm{red}=27^{\circ} \mathrm{C}$ ). Each line represents the curve from the sampling of an individual egg capsule for dorsal mantle length (xaxis). Lines are shaded and patterned to help differentiate individual egg capsules within a plot, but this carries no relationship or meaning across the plots. The filled triangle on the $\mathrm{x}$-axis marks the mean value for the compiled sample of that treatment 

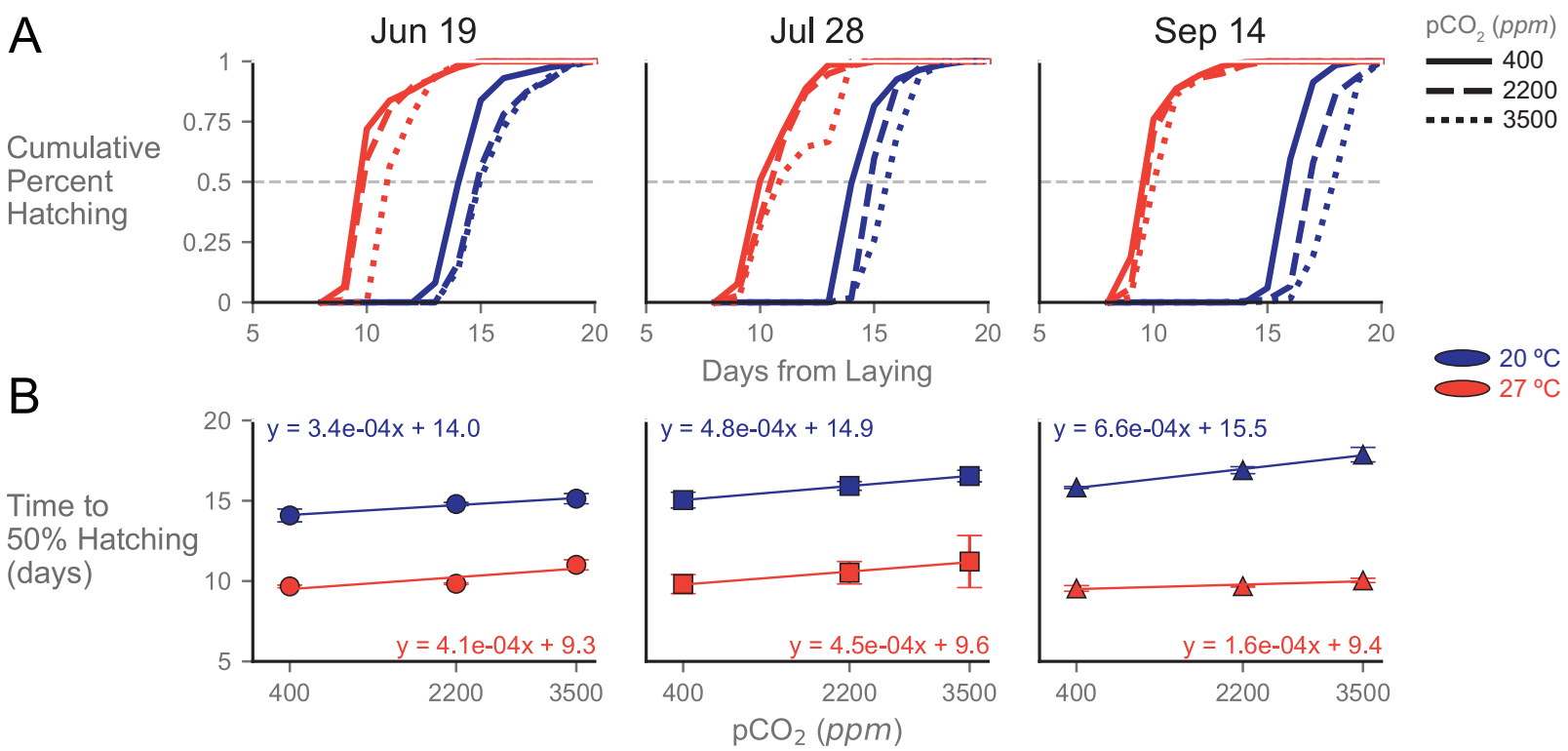

Figure 3. Hatching data from experiments depicted as (A) cumulative hatching curves and (B) plots of time to $50 \%$ hatch. (A) Cumulative hatching curves are shown for each trial (lay date in title). Patterning of lines represents acidification treatments (smaller hash $=$ more acidic), while color represents temperature treatment (blue/dark $=20{ }^{\circ} \mathrm{C}$, red/light $=27^{\circ} \mathrm{C}$ ). Error bars $/$ shading omitted for visual clarity of the curves. The gray dashed line represents the $50 \%$ hatching mark. (B) Time to $50 \%$ hatching data across acidification exposures, calculated from the curves in (A), plotted for each trial. Symbols depict means (and represent trial) with errorbars of one standard deviation (most error ranges are so small that errorbars are contained within the symbol). Color represents temperature treatment as in (A). Regression lines with related equations are presented to visualize trends and assess changes in slope and y-intercept across treatments and trials 

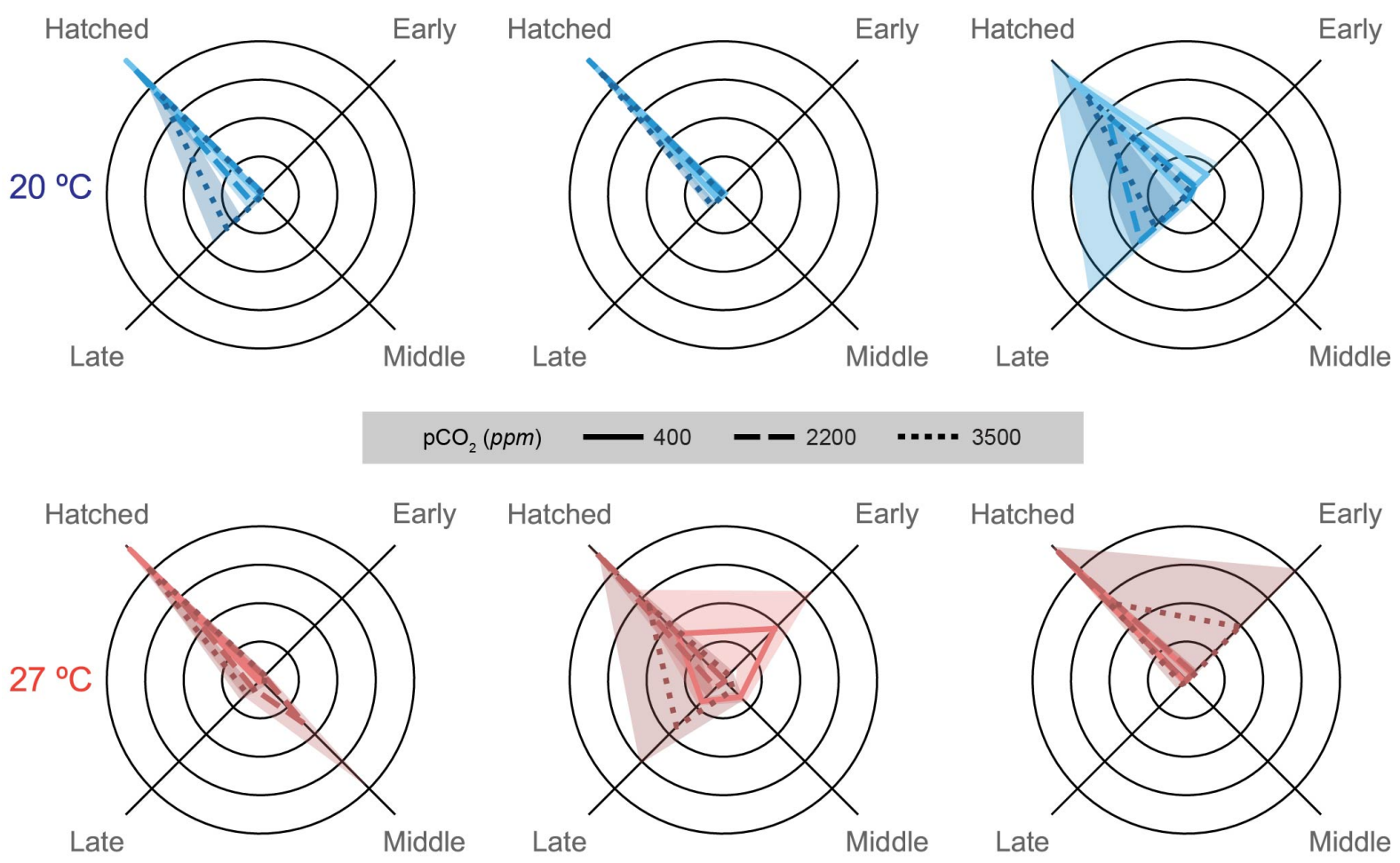

Figure 4. Embryonic survival data depicted for each trial (column title) on spider plots: axes represent categorical variables, while rings represent proportions of embryos in each category (labeled in Jun 19 plots). Categories include, clockwise from top left, Hatched: embryos that successfully hatched from the egg capsules (includes premature and malformed hatchlings), and embryos that ceased development either Early: Arnold stages 1 - 16, Middle: Arnold stages 17 26, or Late: Arnold stages 27 - 30 (Arnold et al., 1974). Lines represent means, while shading represents one standard deviation. Line patterns and shade of color represent acidification treatments (smaller hash $/$ darker color $=$ more acidic). Plot position and data coloration represent temperature treatment (top/blue $=20^{\circ} \mathrm{C}$, bottom $/ \mathrm{red}=27^{\circ} \mathrm{C}$ ) 
Figure 5. (A) Images of types of hatched Doryteuthis pealeii paralarvae corresponding to the axes categories in the data figure (B), as referenced by the 'X.' From top left: Normal: a typical hatchling paralarva, Premature: a paralarva showing remaining external yolk, Eye Bulge: a paralarva with inflation of the membrane around the eye, and Malformed Head: a paralarva with misshapen head, can also present with strange growths or misshapen mantle. All images have a unique $1 \mathrm{~mm}$ white scale line in the bottom left. (B) Malformation data for each trial (column title) on spider plots: axes represent categorical variables, while rings represent proportions of embryos in each category (labeled in Jun 19 plots). Categories include, clockwise from top left, Normal, Premature, Eye Bulge, and Malformed Head as depicted in (A). Lines represent means, while shading represents one standard deviation. Line patterns and shade of color represent acidification treatments ( maller hash/ darker color $=$ more acidic). Plot position and data coloration represent temperature treatment (top $/$ blue $=20^{\circ} \mathrm{C}$, bottom $/ \mathrm{red}=27^{\circ} \mathrm{C}$ ) 


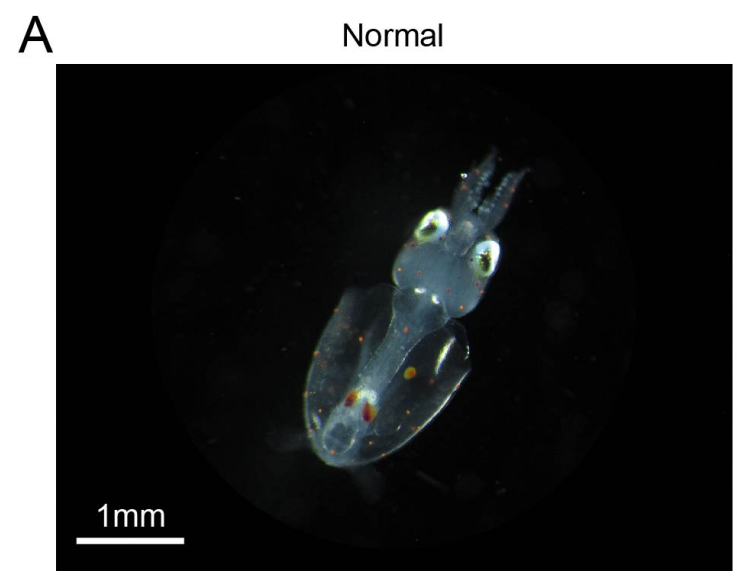

Malformed Head

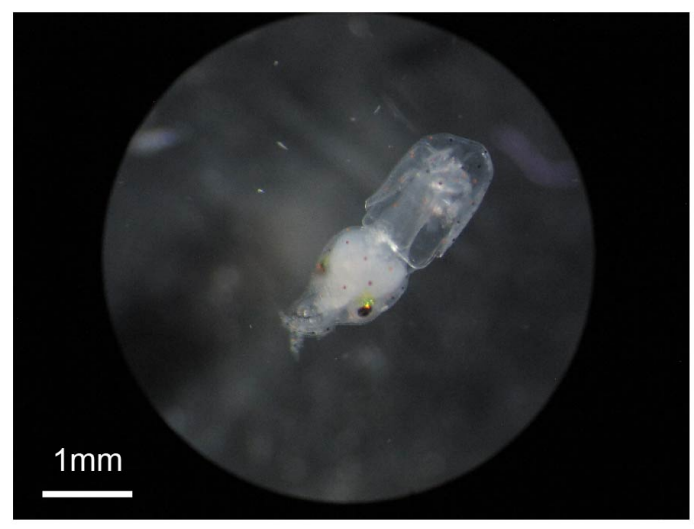

Premature

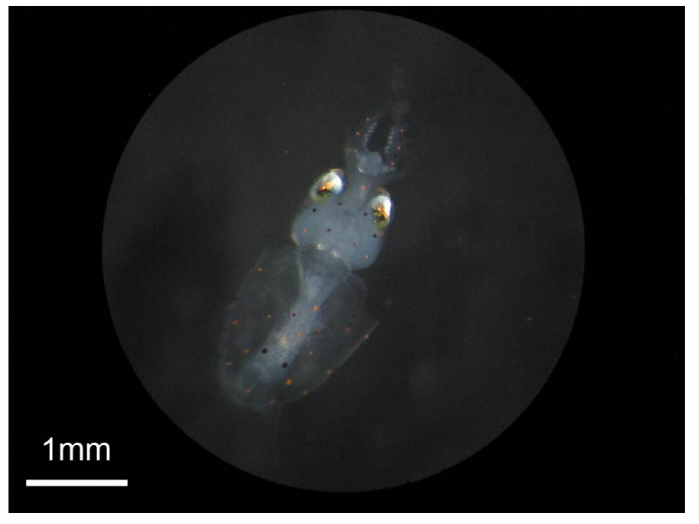

Eye Bulge

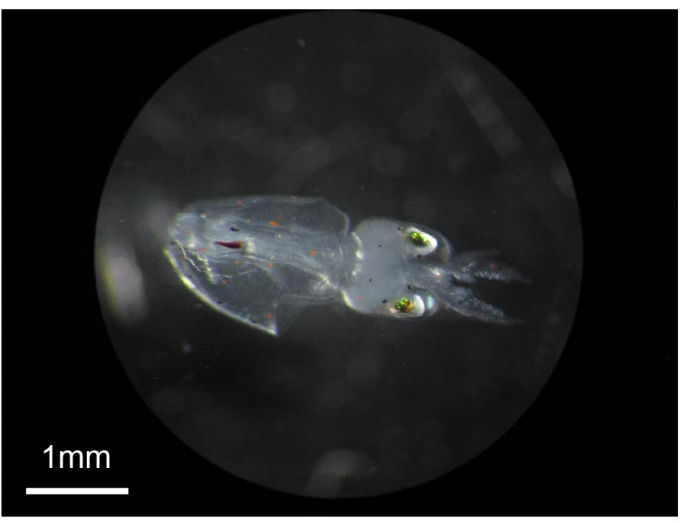

B

Jun 19

Jul 28

Sep 14
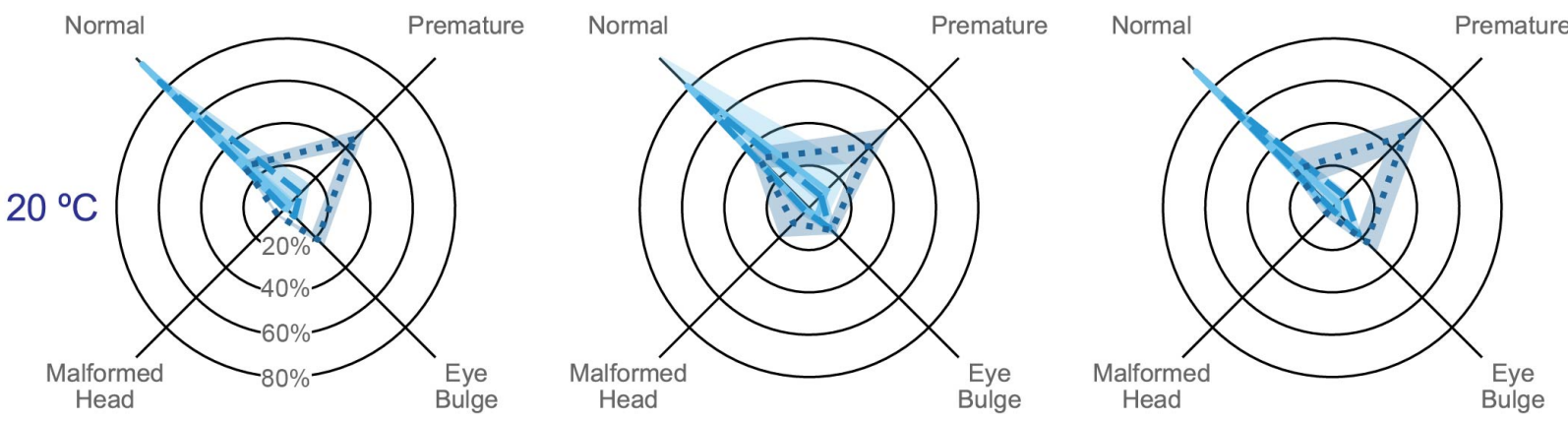

$\mathrm{pCO}_{2}(\mathrm{ppm}) \quad 400 \quad-\quad 2200 \quad \cdots 3500$
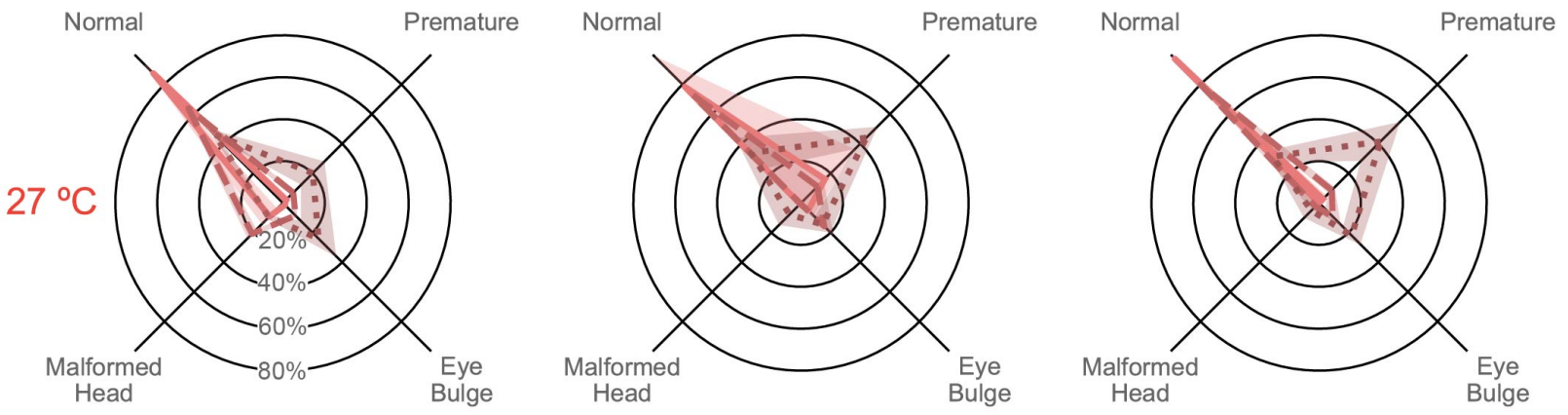


\section{Chapter 6}

\section{Conclusion and Future Directions}

Early squid development under acidification: advances to the conceptual model following this dissertation 


\subsection{Summary}

Coastal systems, particularly those in the northwest Atlantic, are undergoing rapid change due to anthropogenic stressors from industrialization and carbon dioxide emissions, such as ocean acidification and warming (Hobday and Pecl 2014; Pachauri and Meyer 2014; Gledhill et al. 2015). While we still struggle to understand the expected extent of damage already done, it is important that we continue to study what impacts these changes may have on the organisms that support the fisheries and ecosystems on which we rely (Pecl and Jackson 2008; Kroeker et al. 2013; Hobday et al. 2016). Organismal studies of key, representative, and accessible species inform models and provide the foundation for further mechanistic and ecological hypotheses. Performing these studies with a non-model organism that is hard to maintain in aquarium systems is another challenge, but one where because of, rather than in spite of, the mess, there is a lot to learn (Vidal et al. 2014). Cephalopods, and squid in particular, are interesting because they are so plastic and complex and unpredictable. You cannot put a dynamic animal in a dynamic situation and expect straightforward results, but if you stick with it, learn from it, and adapt along with it, some really interesting and valuable patterns can emerge.

This dissertation sought to more deeply examine the impacts of ocean acidification on the early life history of the inshore longfin squid, Doryteuthis pealeii, by measuring metrics of embryonic development and morphology, physiology, and behavior of the hatchling paralarvae. I expanded upon the preliminary data collected by Kaplan et al. (2013) and the early analyses performed in my Master's degree (Zakroff 2013) by developing novel, quantitative methodologies and applying them to measurements of the morphology of paralarvae reared under chronic acidification over a range of $\mathrm{pCO}_{2}$ concentrations. In parallel to that I expanded into behavioral analyses, which constantly evolved in order to try to best capture change in a dynamic and plastic organism. While the squid did not act predictably and replicate the results seen in 2013 in subsequent years, I adapted and shifted experimental treatments and added methodologies (many of which were not successful and are not discussed here, e.g. survival chambers for paralarvae from six multiple stressor treatments was too challenging a system for me to build and maintain on top of everything else) as needed to endeavor to capture what was going on with this squid's remarkable resistance to high levels of acidification and the variability of its sensitivity to OA on larger time scales. And doing so I found interannual patterns of variability, relationships to parental conditioning from the environment, and relationships 
between metrics I would otherwise not have thought to examine. Pushing into multistressor experimentation by adding warming increased the complexity of experiments, but also allowed for some real insights into when and how these stressors affect embryonic development and the state of hatchling paralarvae. In sum, I found that these benthically bound eggs are quite robust in the face of acidification, though there are factors that can cause sensitive clutches through sensitive cohorts/year classes. It is likely then that this population can manage under predicted levels of future change, assuming replete food, but worth considering both that these experiments do not assess the viability of the paralarvae and their ability to reach the juvenile stage and that as warming increases, the likelihood of a particularly OA sensitive year may also increase.

\section{1a The conceptual model when we started}

Prior to beginning this dissertation work, our understanding of acidification impacts on the embryonic development of coastal squid eggs had to be pieced together from a number of sources. What is broadly true among this taxon, the loliginid squids that lay multiple eggs per capsule, is that an individual fertilized egg is packaged with anywhere between 50-300 siblings (sometimes half-siblings because paternity and fertilization is complicated; Arnold et al., 1974; Jacobson, 2005; Buresch et al., 2006). Each embryo is instilled with a certain ration of maternal resources, and it is this maternal input in combination with genetic recombination and epigenetic conditioning that provides the starting point for development (Steer et al. 2004; Jensen et al. 2014). This encapsulated embryo is a discrete energy system, it consumes what is available in the yolk, but cannot intake additional energy from the environment (Villanueva et al. 2011). This limited energy resource must be budgeted amongst needed processes, most prominently development and homeostasis (Sokolova et al. 2012; Sokolova 2013).

What the squid embryo does consume from its environment as it develops is oxygen, and likewise it produces carbon dioxide. Aerobic respiration of the squid and its encapsulated siblings drives down available oxygen and increases acidity within the egg capsule (Long et al. 2016). The degree to which this process makes the embryos surroundings anoxic and acidic may depend greatly upon not only its position within the egg capsule, but also the egg capsule's position with the egg mass, and the seawater chemistry and flow conditions of the laying habitat by affecting the thickness of the boundary layer and the rate of chemical exchange the egg capsule has with its environment (Steer and Moltschaniwskyj 2007; Long et al. 2016; Navarro et 
al. 2018). As the squid embryo develops, it constructs machinery, in the form of proton pumps in ion regulatory epithelia, to manage intracellular $\mathrm{pH}$ as its environment naturally acidifies (Hu et al. 2010).

Under acidification, we presume that the proton concentration gradient between the capsule and its environment is initially reversed and ultimately weakened, such that the capsule acidifies much more quickly, and to a greater extent, during development than it would under ambient conditions. The capsule potentially provides a strong barrier to initial acidification though, and its presence in our experiments likely confers resistance while experiments with embryos that have been removed from the capsule see more direct and immediate stress (Rosa et al. 2014a). Doryteuthis pealeii egg capsules cultured in high dose acidification $\left(2200\right.$ ppm $\left.\mathrm{CO}_{2}\right)$ had been shown to result in delayed hatching, decreases in size of the paralarvae, and degradation of the statoliths (Kaplan et al. 2013). Hatching delays suggest a slowing of the developmental machinery, either because metabolism is being suppressed or available energy is being diverted from processes of development to homeostasis. Decreased size of hatched pararlavae on top of hatching delay, suggests that rather than dampen growth rate, energy contributing to somatic growth is being reduced to compensate for increased strain on homeostatic machinery. Although it is also possible that growth rate is dampened and the delay was not sufficient for size at hatching to reach what it was under control conditions. Changes in the size of statolith are likely driven by changes in the size of the paralarvae (Ikeda et al. 1999; Steer et al. 2003). Degradation of the statolith surface, however, suggests either an acidifying of the statocyst space or a disruption to the production and/or activity of the proteins responsible for constructing the statolith (Lipinski 1993; Cohen and Holcomb 2009). It is from this foundational conceptual model that the experiments described in this dissertation were designed.

\section{1b Dissertation findings}

Chapter 2: Dose-dependence and small-scale variability in responses to ocean acidification during squid, Doryteuthis pealeii, development

The piecewise linear regressions I used in Chapter 2 reinforce the shift in response patterns seen in the 2013 sample at $1300 \mathrm{ppm} \mathrm{CO}_{2}$. This fits with observed impacts to Loligo vulgaris embryos at $\sim 1650 \mathrm{ppm}$ and Doryteuthis opalescens impacts at $\sim 1440 \mathrm{ppm}$, but these are individual data points from experiments that are challenging to compare with this work due to 
differences in stressor exposure and other methodology (Rosa et al. 2014a; Navarro et al. 2016). No other studies that we know of have examined dose response to acidification in cephalopod embryos, so this research serves as a foundation for further research into this stressor for this taxon. This potential threshold for sensitive egg clutches is still remarkably high considering the adults of the species do not spend time in highly $\mathrm{pH}$ variable waters outside of estuaries and the paralarvae are expected to be quite $\mathrm{pH}$ sensitive (Vidal et al. 2002b; Jacobson 2005). This value falls within predictions for open ocean $\mathrm{pH}$ between the years 2100 and 2250, but can observed in coastal systems on short, variable timescales (Caldeira and Wickett 2003; Baumann et al. 2015; Gledhill et al. 2015). This suggests that in the immediate scope, acidification may not be the highest concern for Doryteuthis pealeii egg laying habitat, compared to warming, hypoxia, and other more imminent, potentially more impactful stressors; although it should be kept in mind that OA may contribute to interactions with these more dominant stressors.

The responses seen in these data reflect those seen in the preliminary Kaplan et al. (2013) study, but provide a richer picture with the addition of hatching success, yolk volume, and quantitative statolith analyses. In particular, the comparison of mantle length and yolk volume provides insight into the possible physiological and energy budget management strategies available to the squid embryos. I found that portfolio of possible strategies to be pretty diversified, as response intensities and corresponding yolk consumption vs. growth strategies shifted across the breeding season. I also note shifts in these dynamics over the course of hatching, providing insight into both a new dimension of hatching variability and trends in response that further inform possible physiological and metabolic strategies (e.g. a decrease in both yolk and size over hatching suggests a stressed system that has to sacrifice somatic growth and tap additional resources to reach a viable hatching state). This lends credence to the potential for seasonal effects or microcohorts among this population of squid (Moltschaniwskyj and Pecl 2007; Murray et al. 2014; Rosa et al. 2014a). It also suggests that within a breeding season, a diversity of responses are common allowing for differential success under varying conditions, which likely hedges bets for population success (Pecl and Jackson 2008; Crean and Marshall 2009; Hoving et al. 2013).

Chapter 3 - Ocean acidification responses in paralarval squid swimming behavior using a novel $3 D$ tracking system 
Given the presence of statolith impacts in Kaplan et al. (2013) and in the data from Chapter 2, there was strong interest in seeing if these impacts translated to the swimming behavior of the hatchling paralarvae. In Chapter 3, I ran experiments in 2013 in a 2D, primarily vertical, arena with paralarvae from the sensitive system described above. I found that paralarvae were less able to maintain station near the surface, they sank more than they hopped, with increased acidification, but the system did not allow for the robust examination of orientation and turning I had intended. I therefore developed a 3D arena and organismal position model system to collect better data to address these hypotheses, and implemented this system in 2014 and 2015, when paralarvae no longer showed the sensitivities or statolith impacts under acidification they had prior. Still, I found, using this system, decreases to 3D velocity and swimming activity of the paralarvae under acidification, even as relatively low as $1000 \mathrm{ppm}$ $\mathrm{CO}_{2}$. This suggests that the paralarvae might indeed show greater sensitivity to chronic $\mathrm{pH}$ exposure than the encapsulated, developing embryos do (Vidal et al. 2002b, 2014; Robin et al. 2014). Further, it is a concern for survival if paralarvae cannot maintain position, as part of placing themselves in desired currents and performing vertical migrations, or swim as quickly, both as fledgling predators and potential prey (Martins et al. 2010a; York and Bartol 2016; York et al. 2016). Substantive individual variability, however, suggests that paralarvae survival may hinge on a scattershot, somewhat luck-based approach, although even slight reductions to overall cohort survival could translate to population scale impacts (Robin et al. 2014; Doubleday et al. 2016).

Chapter 4 - Interannual and seasonal variability in the response of squid embryos and paralarvae reared under ocean acidification

In Chapter 4 I highlight the shifts in OA sensitivity across all four years of experiments (and the preliminary work by Kaplan et al., 2013). Response ratios provide a discrete and efficient means to compare effect size across years and metrics; while they are typically used for meta-analyses, using them to synthesize across the common acidification exposure used across experiments (2200 ppm $\mathrm{CO}_{2}$ ) here bore a range of insights (Kroeker et al. 2013; Baumann et al. 2018). I describe variability in acidification response between and within years, showing that, indeed, 2011 (the preliminary work), 2013, and 2016 showed sensitive trials while 2014 and 2015 did not. Further, I found that response intensity shifts across the season within a sensitive 
year, but with no consistent pattern, suggesting something unique about the tails of the season compared to its peak. I also highlight shifting baselines throughout the breeding season, which are strongly correlated to ambient temperature conditioning of the parents, although these effects do not appear to affect acidification response intensities. These data further support the potential for microcohorts, and could be explained by the model of two year old squid making up the breeding population at the start of the season and one year old squid making up the breeding population at the tail of the season (Arnold et al. 1974; Mesnil 1977; Moltschaniwskyj and Pecl 2007).

Using response ratios, I also uncovered previously undescribed relationships between the metrics I measured in the squid. Dorsal mantle length and hatching time correlated with one another, and these metrics varied on the interannual scale. Yolk volume and hatching success, on the other hand, correlated with each other, with responses in these metrics varying within years, across the breeding season. I found that embryos that consumed relatively more yolk during development were more likely to hatch, and that these were the embryos that had relatively more yolk at hatching under control conditions. Therefore, within a season, maternal condition and rationing to eggs is key to hatching success under stress (Steer et al. 2004). Impacts to growth and development, metabolic responses, are seemingly driven instead by environmental conditioning of the parental year class (Pecl et al. 2004a; Murray et al. 2014; Putnam and Gates 2015). I hypothesize that these annual and seasonal sensitivities relate broadly to the overwintering temperatures of the squid year class, with anomalously warmer years resulting in greater sensitivity. This indicates that management of the squid population hinges on a better understanding of the factors of environmental history that define epigenetic transference, abundance, and population success in cephalopods (Yatsu et al. 2000; Pierce and Boyle 2003; Roberts 2005). These results also suggest that while the eggs of Doryteuthis pealeii are relatively robust under acidification, there may be cause for concern under a combined warming and acidification scenario.

Chapter 5 - Antagonistic interactions and clutch-dependent sensitivity induce variable responses to ocean acidification and warming in squid (Doryteuthis pealeii) embryos

In 2016, I combined acidification with warming to increase stresses upon the developing squid eggs. Unsurprising by this point, but still novel, in Chapter 5 I found different sensitivities 
and response strategies to both stressors across this breeding season. Surprisingly, I found that warming appeared to either dominate or act antagonistically to acidification impacts rather than compound or amplify them as has been seen in other species (Rosa et al. 2014a). Warming has been shown to be a dominating stressor in larvae of other ectothermic taxa, so perhaps this result is not quite so shocking (Nguyen et al. 2012). Here, it appears that since warming drives embryonic development to occur much faster, the developing embryos reduce their exposure time to the acidification. This indicates that under a warming and acidification scenario, warming could mitigate acidification impacts, but raises concerns about the changes to the viability and life history strategies of the hatched squid due to the increased rearing temperatures (Keyl et al. 2011; Pimentel et al. 2012; Hoving et al. 2013). On top of this, there is likely a sharp limit to the temperature at which these eggs can successfully develop, as is known in other squids, which would be important to determine for a viable egg habitat window (Sen 2005; Zeidberg et al. 2011).

The other unique factor of Chapter 5 is that each trial was run with egg capsules from a single female squid. While the experiments were not robust or replicated enough to discriminate differences between maternity and seasonality, I could examine the variability in stress response within a clutch of eggs. For dorsal mantle length, I found that egg capsules within a clutch under control conditions all respond quite similarly with tight, relatively tall bell curves around a mean paralarval size. Slight stress appears to simply cause this mean to shift, while severe stress causes this mean shift and an increase in variability, resulting in a flattening of the curve. I found that egg capsule under stress diverge in their responses, suggesting that while maternity may set the baseline state, paternity/the recombinant genetic background may play a role in response under stress (Buresch et al. 2001; Marshall 2015; Guillaume et al. 2016; Bonduriansky and Crean 2018).

\section{1c The conceptual model after this dissertation}

How, then, has our understanding of this system, D. pealeii egg capsules reared under acidification (and warming), changed as a consequence of this dissertation? Much of the foundation leading to the egg being laid remains the same, but we must now purposefully consider both the environmental history of the parents and the ambient seasonal temperature in assuming some combination of maternal investment, genetic predisposition, and epigenetic 
conditioning being transmitted to the fertilized egg. If those factors are such that a year class and clutch will be sensitive to acidification, we expect that under doses of $1300 \mathrm{ppm} \mathrm{CO}_{2}$ or greater, the egg capsule acidifies enough in late development to cause stress. The embryos may then respond by either consuming more available yolk (more likely if more yolk is initially available, which is seasonally dependent), suppressing development and metabolism, sacrificing energy from somatic growth (likely both of these latter options in sensitive years), or some combination of any of the three.

If warming is co-occurring in our hypothetical system, embryos will develop faster, increasing metabolism and decreasing time to hatching. On its own, this can have dramatic effects on the viability of the embryos from the outset of development, resulting in disrupted growth and malformation. Warming drives increased aerobic respiration, which can expedite hypoxic and anoxic conditions in the egg capsules resulting in a greater hatch of premature paralarvae (Pimentel et al. 2012; Rosa et al. 2012). Increased temperature, however, also functionally reduces the exposure time of embryos under acidification, thus slightly reducing acidification impacts in late stage development.

Slight decreases and surface degradations in statoliths caused by acidification do not appear to disrupt their functionality enough to impair paralarval swimming. However, paralarvae, lacking the protections of the egg capsule, may show metabolic suppression via decreased swimming activity and speed at much lower doses of acidification $\left(1000 \mathrm{ppm} \mathrm{CO}_{2}\right.$ or above). The full range of impacts due to the transition from embryo to paralarvae under these conditions remains unclear.

\subsection{Future directions}

Several datasets remain to be processed from this dissertation concerning topics ranging from statolith morphometrics and relative density analysis, to phototactic behavior, to gene expression. Beyond these data, there is still much to uncover in regards to squid embryo tolerance, epigenetic transference of resistance, and the role of encapsulation and plasticity in dealing with rapidly changing and variable environments (Noisette et al. 2014). The exposures used here were all chronic and constant whereas in a natural system they would vary on daily, tidal, and seasonal cycles (Baumann et al. 2015; Gledhill et al. 2015). It would be worth investigating if encapsulated eggs and hatchling paralarvae do better or worse in these varying 
conditions. The model of an egg being more robust under stress, due to its developmental machinery and its physical protections, than the resultant larvae is one that is intuitive, but not robustly studied across taxa; and little is known about the physiological transitions that occur here in squid naturally, much less under stress (Noisette et al. 2014; Robin et al. 2014). A better understanding of the viability of the paralarvae and their ability to transition into juveniles under stress is vital to informing strong predictive models of population success for management of this species. Given these studies as a framework, it would be worth expanding into other, potentially more impactful stressors, such as hypoxia and pollutants (Lacoue-Labarthe et al. 2008; Navarro et al. 2016). While $D$. pealeii is not yet in a place where it can be viably and sustainably reared in aquaculture, many bobtail squids are, so transgenerational studies in cephalopods to examine the epigenetic mechanisms hinted at in this dissertation are becoming more and more possible.

\subsection{Broader implications}

Our global ocean is changing rapidly due to human activity and it is vital that science keep pace by assessing potential impacts across stressors and taxa (Crain et al. 2008; Pachauri and Meyer 2014; Kroeker et al. 2017). This drive to rapidly produce key multistressor studies with key organisms has led to a large number of individual, novel experiments, with results that, while valuable, are not always substantiated by replication. Few organismal stress studies are repeated in earnest, fewer still over significant time periods, but those that do can demonstrate large scale trends and temporal variability that is deeply relevant to predicting population survival under ocean change (Kroeker et al. 2017; Baumann et al. 2018). This dissertation, beyond its specific utility in better understanding squid development and stress physiology and the potential impacts of ocean acidification, serves as a representation of the importance and need for longer term, repeated studies and of the value in collecting and robustly analyzing complicated results as part of describing the natural complexity and variability of an organism. 


\section{Appendix A}

\section{Chapter 2 Supplementary Materials}

This chapter was originally published as: Zakroff C, Mooney TA, Berumen ML (2019) Dose-dependence and smallscale variability in responses to ocean acidification during squid, Doryteuthis pealeii, development. Marine Biology 166:62. doi: 10.1007/s00227-019-3510-8. 
Table S1. One-way ANOVAs of $\mathrm{pCO}_{2}$ separated by metric and trial; significant $\mathrm{p}$-values $(<0.05)$ in bold. Yolk volume measurements were log-transformed prior to statistical analysis. Compiled data is difference from trial mean.

\begin{tabular}{|c|c|c|c|c|c|c|c|c|c|c|}
\hline \multirow[b]{2}{*}{ Source } & \multicolumn{5}{|c|}{ Mantle Length } & \multicolumn{5}{|c|}{ Yolk Sac Volume } \\
\hline & SS & Df & $F$ & $p$ & $\Omega^{2}$ & SS & $d f$ & $F$ & $p$ & $\Omega^{2}$ \\
\hline \multicolumn{11}{|l|}{ Jul 3} \\
\hline $\mathrm{pCO}_{2}$ & 0.573 & 2 & 20.98 & $<0.001$ & 0.079 & 5.604 & 2 & 9.10 & $<0.001$ & 0.038 \\
\hline Residual & 6.305 & 462 & & & & 125.9 & 409 & & & \\
\hline \multicolumn{11}{|l|}{ Jul 11} \\
\hline $\mathrm{pCO}_{2}$ & 1.035 & 2 & 38.27 & $<0.001$ & 0.132 & 2.480 & 2 & 3.38 & 0.035 & 0.010 \\
\hline Residual & 6.598 & 488 & & & & 180.6 & 492 & & & \\
\hline \multicolumn{11}{|l|}{ Aug 7} \\
\hline $\mathrm{pCO}_{2}$ & 0.297 & 2 & 9.24 & $<0.001$ & 0.032 & 0.703 & 2 & 2.19 & 0.113 & 0.005 \\
\hline Residual & 7.867 & 490 & & & & 76.52 & 477 & & & \\
\hline \multicolumn{11}{|c|}{ Compiled } \\
\hline $\mathrm{pCO}_{2}$ & 1.568 & 5 & 21.43 & $<0.001$ & 0.066 & 4.243 & 5 & 3.02 & 0.010 & 0.007 \\
\hline Residual & 21.11 & 1443 & & & & 387.6 & 1381 & & & \\
\hline
\end{tabular}


Table S2. One-way ANOVAs of hatching date separated by metric, trial, and $\mathrm{pCO}_{2}$ treatment; significant $\mathrm{p}$-values $(<0.05)$ in bold. Yolk volume measurements were log-transformed prior to statistical analysis.

\begin{tabular}{|c|c|c|c|c|c|c|c|c|c|c|c|}
\hline \multirow[b]{2}{*}{ Trial } & \multirow[b]{2}{*}{ Source } & \multicolumn{5}{|c|}{ Mantle Length } & \multicolumn{5}{|c|}{ Yolk Sac Volume } \\
\hline & & SS & df & $F$ & $\mathrm{p}$ & $\Omega^{2}$ & SS & df & $F$ & $\mathrm{p}$ & $\Omega^{2}$ \\
\hline \multirow{9}{*}{ Jul 3} & \multicolumn{11}{|c|}{ ESL Ambient } \\
\hline & Date & 0.071 & 5 & 1.219 & 0.303 & 0.007 & 1.396 & 5 & 0.788 & 0.560 & -0.007 \\
\hline & Residual & 1.816 & 156 & & & & 52.772 & 149 & & & \\
\hline & \multicolumn{11}{|l|}{1300 ppm } \\
\hline & Date & 0.037 & 5 & 5.771 & $<0.001$ & 0.141 & 9.624 & 5 & 10.429 & $<0.001$ & 0.280 \\
\hline & Residual & 1.756 & 139 & & & & 21.224 & 115 & & & \\
\hline & \multicolumn{11}{|l|}{2200 ppm } \\
\hline & Date & 0.153 & 5 & 2.168 & 0.061 & 0.036 & 6.870 & 4 & 6.616 & $<0.001$ & 0.142 \\
\hline & Residual & 2.145 & 152 & & & & 34.007 & 131 & & & \\
\hline \multirow{9}{*}{ Jul 11} & \multicolumn{11}{|l|}{850 ppm } \\
\hline & Date & 0.286 & 5 & 4.666 & $<0.001$ & 0.096 & 9.343 & 5 & 6.727 & $<0.001$ & 0.152 \\
\hline & Residual & 2.038 & 166 & & & & 42.780 & 154 & & & \\
\hline & \multicolumn{11}{|l|}{1300 ppm } \\
\hline & Date & 0.351 & 5 & 6.446 & $<0.001$ & 0.135 & 1.878 & 5 & 1.290 & 0.271 & 0.009 \\
\hline & Residual & 1.839 & 169 & & & & 46.606 & 160 & & & \\
\hline & \multicolumn{11}{|l|}{2200 ppm } \\
\hline & Date & 0.300 & 4 & 5.843 & $<0.001$ & 0.119 & 8.603 & 5 & 3.926 & 0.002 & 0.080 \\
\hline & Residual & 1.784 & 139 & & & & 71.438 & 163 & & & \\
\hline \multirow{9}{*}{ Aug 7} & \multicolumn{11}{|l|}{400 ppm } \\
\hline & Date & 0.169 & 5 & 2.202 & 0.057 & 0.036 & 3.813 & 5 & 5.519 & $<0.001$ & 0.124 \\
\hline & Residual & 2.382 & 155 & & & & 21.141 & 153 & & & \\
\hline & \multicolumn{11}{|l|}{1900 ppm } \\
\hline & Date & 0.426 & 5 & 5.653 & $<0.001$ & 0.125 & 1.214 & 5 & 1.430 & 0.216 & 0.010 \\
\hline & Residual & 2.365 & 157 & & & & 26.310 & 155 & & & \\
\hline & \multicolumn{11}{|l|}{2200 ppm } \\
\hline & Date & 0.605 & 5 & 10.267 & $<0.001$ & 0.215 & 4.229 & 5 & 6.575 & $<0.001$ & 0.148 \\
\hline & Residual & 1.920 & 163 & & & & 19.812 & 154 & & & \\
\hline
\end{tabular}


Table S3. One-way ANOVAs of culture cup separated by metric, trial, and $\mathrm{pCO}_{2}$ treatment; significant $\mathrm{p}$-values $(<0.05)$ in bold. Yolk volume measurements were log-transformed prior to statistical analysis.

\begin{tabular}{|c|c|c|c|c|c|c|c|c|c|c|c|}
\hline \multirow[b]{2}{*}{ Trial } & \multirow[b]{2}{*}{ Source } & \multicolumn{5}{|c|}{ Mantle Length } & \multicolumn{5}{|c|}{ Yolk Sac Volume } \\
\hline & & SS & df & $F$ & $\mathrm{p}$ & $\Omega^{2}$ & SS & df & $F$ & $\mathrm{p}$ & $\Omega^{2}$ \\
\hline \multirow{9}{*}{ Jul 3} & \multicolumn{11}{|c|}{ ESL Ambient } \\
\hline & Cup & 0.113 & 2 & 5.059 & 0.007 & 0.048 & 7.538 & 2 & 12.286 & $<0.001$ & 0.127 \\
\hline & Residual & 1.774 & 159 & & & & 46.629 & 152 & & & \\
\hline & \multicolumn{11}{|l|}{1300 ppm } \\
\hline & Cup & 0.258 & 2 & 9.851 & $<0.001$ & 0.109 & 0.452 & 2 & 0.877 & 0.419 & -0.002 \\
\hline & Residual & 1.863 & 142 & & & & 30.396 & 118 & & & \\
\hline & \multicolumn{11}{|l|}{2200 ppm } \\
\hline & Cup & 0.033 & 2 & 1.113 & 0.331 & 0.001 & 0.323 & 2 & 0.530 & 0.590 & -0.007 \\
\hline & Residual & 2.265 & 155 & & & & 40.553 & 133 & & & \\
\hline \multirow{9}{*}{ Jul 11} & \multicolumn{11}{|l|}{850 ppm } \\
\hline & Cup & 0.201 & 2 & 8.014 & $<0.001$ & 0.075 & 0.804 & 2 & 1.231 & 0.295 & 0.003 \\
\hline & Residual & 2.123 & 169 & & & & 51.318 & 157 & & & \\
\hline & \multicolumn{11}{|l|}{1300 ppm } \\
\hline & Cup & 0.048 & 2 & 1.937 & 0.147 & 0.011 & 1.323 & 2 & 2.287 & 0.105 & 0.015 \\
\hline & Residual & 2.142 & 172 & & & & 47.161 & 163 & & & \\
\hline & \multicolumn{11}{|l|}{2200 ppm } \\
\hline & Cup & 0.148 & 2 & 5.380 & 0.006 & 0.057 & 6.832 & 2 & 7.745 & $<0.001$ & 0.074 \\
\hline & Residual & 1.936 & 141 & & & & 73.210 & 166 & & & \\
\hline \multirow{9}{*}{ Aug 7} & \multicolumn{11}{|l|}{400 ppm } \\
\hline & Cup & 0.289 & 2 & 10.105 & $<0.001$ & 0.102 & 2.038 & 2 & 6.938 & 0.001 & 0.070 \\
\hline & Residual & 2.262 & 158 & & & & 22.915 & 156 & & & \\
\hline & \multicolumn{11}{|l|}{1900 ppm } \\
\hline & Cup & 0.437 & 2 & 14.853 & $<0.001$ & 0.145 & 1.392 & 2 & 4.209 & 0.017 & 0.038 \\
\hline & Residual & 2.354 & 160 & & & & 26.131 & 158 & & & \\
\hline & \multicolumn{11}{|l|}{2200 ppm } \\
\hline & Cup & 0.068 & 2 & 2.283 & 0.105 & 0.015 & 0.503 & 2 & 1.677 & 0.190 & 0.008 \\
\hline & Residual & 2.458 & 166 & & & & 23.539 & 157 & & & \\
\hline
\end{tabular}


Table S4. Counts of staged, failed embryos and hatchling paralarvae, compiled by treatment. Failed embryos were staged as early (embryonic stages $1-16)$, middle $(17-26)$, and late (27 - 30). G-tests of hatching distributions are reported with significant $p$-values $(p<0.05)$ in bold. Since all G-tests were significant, exponents of the $p$-value are listed to compare degrees of significance.

\begin{tabular}{|c|c|c|c|c|c|c|c|c|c|c|}
\hline Treatment $\mathrm{pCO}_{2}$ & Early & Middle & Late & Hatched & $\begin{array}{l}\text { Percent } \\
\text { Hatched }\end{array}$ & Source & G & $d f$ & $\mathrm{p}$ & $\exp$ \\
\hline \multicolumn{11}{|l|}{ Jul 3} \\
\hline ESL Ambient & 149 & 9 & 0 & 948 & 85.71 & ESL x 1300 & 162.857 & 3 & $<0.001$ & -35 \\
\hline 1300 ppm & 13 & 0 & 2 & 1117 & 98.67 & ESL x 2200 & 32.510 & 3 & $<0.001$ & -07 \\
\hline 2200 ppm & 87 & 0 & 2 & 993 & 91.77 & $1300 \times 2200$ & 67.514 & 2 & $<0.001$ & -15 \\
\hline & & & & & & All & 171.110 & 6 & $<0.001$ & -34 \\
\hline \multicolumn{11}{|l|}{ Jul 11} \\
\hline 850 ppm & 25 & 6 & 0 & 814 & 96.33 & $850 \times 1300$ & 19.027 & 2 & $<0.001$ & -05 \\
\hline 1300 ppm & 44 & 33 & 0 & 940 & 92.43 & $850 \times 2200$ & 51.866 & 2 & $<0.001$ & -12 \\
\hline 2200 ppm & 26 & 61 & 0 & 796 & 90.15 & $1300 \times 2200$ & 15.649 & 2 & $<0.001$ & -04 \\
\hline & & & & & & All & 56.021 & 4 & $<0.001$ & -11 \\
\hline \multicolumn{11}{|l|}{ Aug 7} \\
\hline 400 ppm & 10 & 7 & 20 & 835 & 95.76 & $400 \times 1900$ & 13.566 & 3 & 0.003 & -03 \\
\hline 1900 ppm & 10 & 27 & 16 & 799 & 93.89 & $400 \times 2200$ & 13.902 & 3 & 0.003 & -03 \\
\hline 2200 ppm & 13 & 11 & 4 & 922 & 91.77 & $1900 \times 2200$ & 18.520 & 3 & $<0.001$ & -04 \\
\hline & & & & & & All & 30.544 & 6 & $<0.001$ & -05 \\
\hline
\end{tabular}




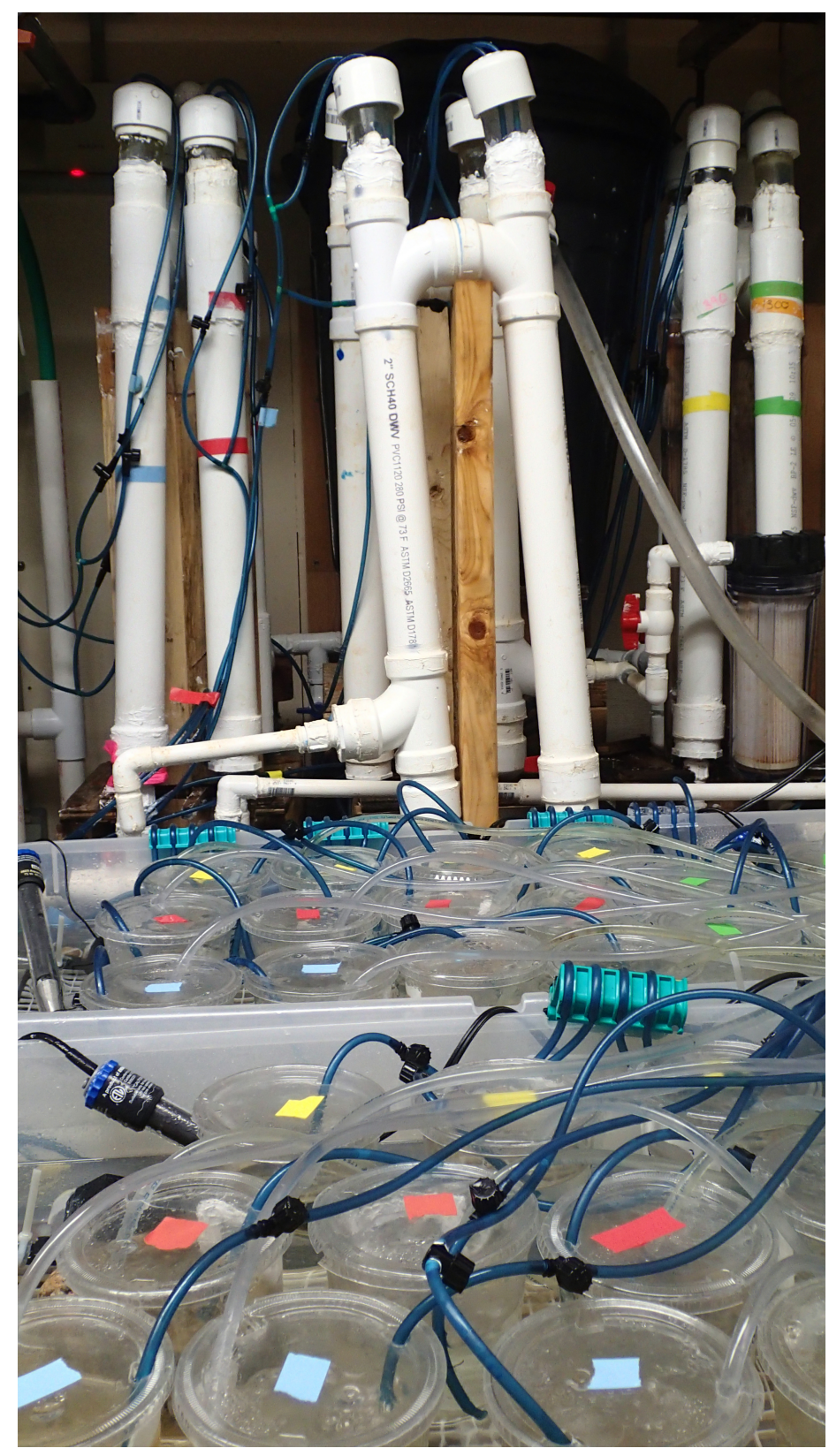

Figure S1. Acidification and egg culture system. Filtered, temperature controlled water from Vineyard Sound was piped into a header tank (behind PVC towers depicted) and flowed into PVC towers where airstones equilibrated water to the desired $\mathrm{pCO}_{2}$ levels. Water exiting the chamber was split in a manifold (not depicted), which fed drip lines into the egg culture cups of each water bath 


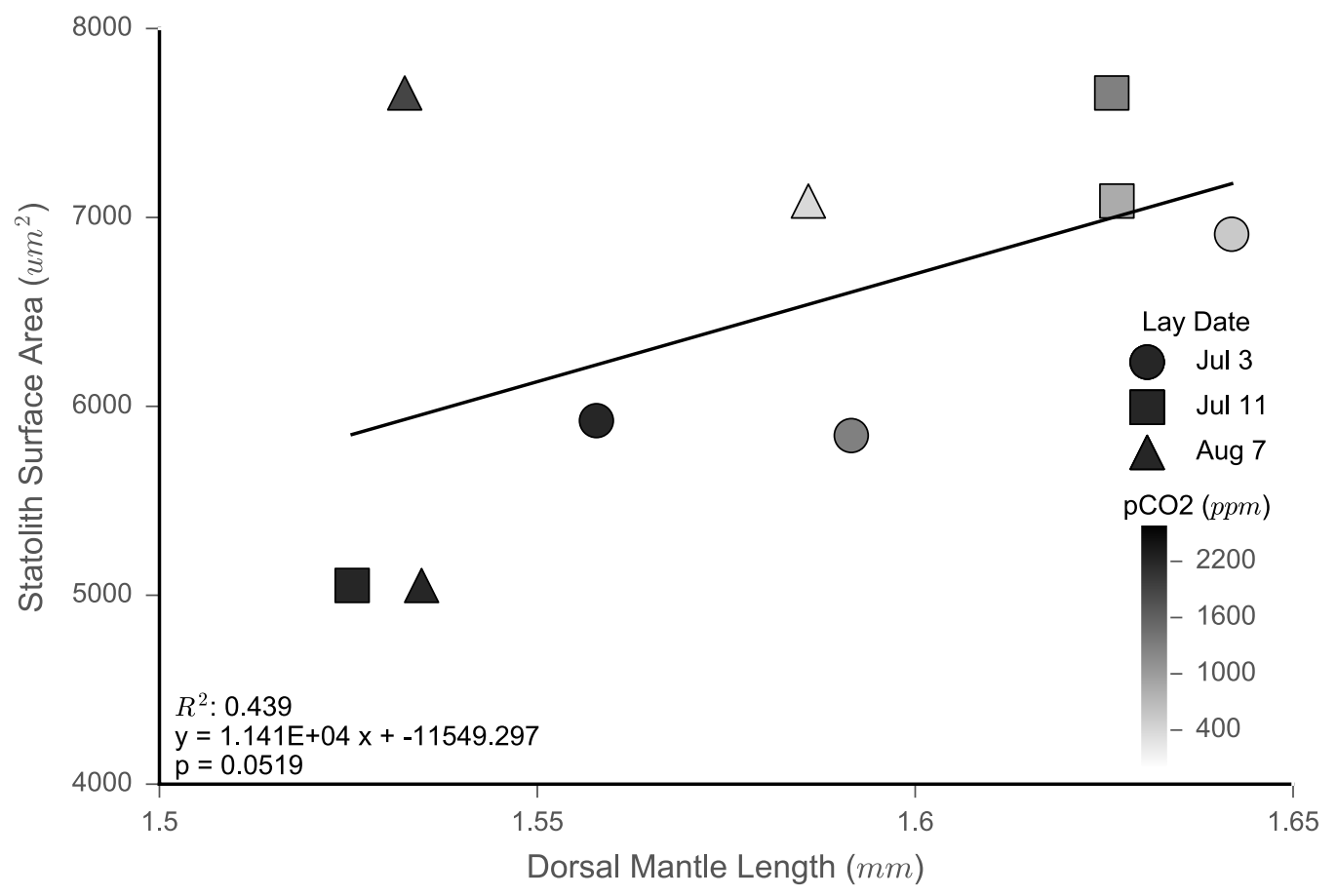

Figure S2. Comparison of average statolith surface area and average mantle length of experimental paralarvae. Data are compiled within each treatment and all trials are plotted together. Symbols represent means, with shape corresponding to trial, and color corresponding to $\mathrm{pCO}_{2}$ treatment (according to the color bar at right). The line depicts a linear regression, with $\mathrm{R}^{2}$, equation, and $\mathrm{p}$ value reported in the lower left corner of the plot 


\section{A1. Morphometric analysis of statoliths}

\section{A1.1a Statolith morphometrics protocol \& $R$ code}

The following R (Version 3.3.3) code was implemented within an R Notebook (StatolithMorphometrics.rmd; available at github.com/czakroff/Statoliths) within RStudio (Version 1.0.136 for Mac OS X). It provides the method for analyzing the silhouetted statolith (black statolith on white background) JPEG's produced in Adobe Photoshop (as described in the main text). This program requires a CSV of metadata with at least the following columns (presumably there will be other relevant sample data as well):

1. Path (path in your system to folder containing the silhouetted images)

2. PicName (image names/ID's of your samples)

Note: The program is written to access a metadata CSV where the PicName column refers to the original SEM TIFF image. This is why the "\#Rename Files" block removes the ".tif" and adds "_BW.jpg." This step can be removed if the PicName column in the metadata refers directly to your silhouetted JPEG's.

The program accesses the silhouetted statolith JPEG's provided by the metadata CSV to build Outline objects using the Momocs package (Bonhomme et al., 2013), which are then analyzed for basic morphometrics as well as the additional metric of rugosity (internal angle variance) developed as part of this manuscript. A few notes on the operation of this code:

- The coo_alignxax method horizontally flips the alignment of the statoliths (they are set with dome pointing right and toward the top of the image and wing pointing left during Photoshop processing). It doesn't affect the outcome, but is important to note.

- The Momocs package contains a number of additional, more complex methods for visualizing and analyzing the shape of objects (elliptical fourier analysis, for instance), which may be worth adding to your analysis depending on the questions of your study.

- The 150 point resolution was determined through an assessment of a sample of 50 test shapes (Fig. S3) and is discussed in detail following the code. 
$--$

title: "Statolith Morphometrics"

output: html_notebook

$---$

This R notebook contains the basic methods to read in and analyze the morphometrics of squid statoliths using the Momocs package (Bonhomme et al., 2013). In addition, the code for calculating the rugosity of the statolith edge (the variance of the internal angles of the outline) is included. The program uses a metadata CSV file (you must supply the PATH to this file) to access the paths and filenames of your image samples (silhouetted JPEGs: black statolith on white background) and then processes the outlines through Momocs to get the basic morphometrics (area, rectangularity, circularity, length, width, and length:width) as well as the rugosity and then outputs this data to a CSV containing an appended version of the original metadata table (you must supply the PATH and name of this file).

More on Momocs can be found at:

Bonhomme, V., Picq, S., Gaucherel, C. and Claude, J. (2013). Momocs: outline analysis using R. J. Stat. Softw. 56, $1-24$.

Version 1.1 written by Casey Zakroff (czakroff@whoi.edu) May 32018

in R Version 3.3.3 on Mac. Code and protocols available at:

https://github.com/czakroff/Statoliths

\#\#\#Step 1

Turn on X11! (Required for running in Mac)

\#\#\#Process statoliths for outlines

"' $\{\mathrm{r}\}$

\#Add Momocs to your active library

library(Momocs)

$\cdots\{r\}$

\#Load in statolith metadata (add your own path \& filename)

data $<$ - read.csv('path/filename.csv') \#read in metadata CSV

path $<$ - as.character(data \$Path) \#read path column for statolith silhouettes

samples $<$ - as.numeric(length(path)) \#number of samples

‥

" $\{r\}$

\#Set Pixel to Micrometer Ratio

$\mathrm{cf}<-6$ \#conversion factor $=6 \mathrm{px} / \mathrm{um}$ for my data. Depends on magnification of your images (but should all be the same).

$\cdots$

$\cdots\{r\}$

\#Rename Files

$\mathrm{x}<-\mathrm{c}()$ \#temporary array for PicNames

for (i in data $\$$ PicName) \{

$\mathrm{p}<$ - substring(i, 1, nchar(i)-4) \#put String in p, but remove ".tif" from SEM image filename

p <- paste (p, '_BW.jpg', sep = "') \#adds silhouette JPEG file ending

$\mathrm{x}<-\mathrm{c}(\mathrm{x}, \mathrm{p})$ \#add to temporary array

\}

data\$PicName $<-\mathrm{x}$ \#reassign to PicName column in image metadata 
$\cdots\{r\}$

\#Write Statolith Outlines

statolith <- import_jpg(paste $($ path,data\$PicName, sep = "')) \#read in all statolith images

stato <- Out(statolith,factor(data\$pCO2)) \#build Outline objects

$\cdots$

$\cdots\{r\}$

\#Optional: view statolith outlines

panel(stato)

$\cdots\{r\}$

\#Reorganize Statoliths

stato_align $<-$ coo_alignxax(stato) \#align along x-axis

stato_align $<-$ coo_center(stato_align) \#center all outlines

stato_align $<-$ coo_slidedirection(stato_align, "N") \#place start point of outline at top ,

$\cdots\{r\}$

\#Optional: view all aligned statoliths superimposed

stack(stato_align)

\#Or view as panel

\#panel(stato_align)

$\cdots$

\#\#\#Basic Morphometrics

' $\{\mathrm{r}\}$

\#Get basic morphometrics

mets <- measure(stato_align, coo_area, coo_circularity, coo_rectangularity) \#measure area, circularity, and rectangularity

$\mathrm{df}<$ - data.frame(matrix(unlist(mets), nrow $=$ samples, byrow $=\mathrm{F})$ ) \#store in temporary dataframe

lw $<-$ coo_lw(stato_align) \#measure length and width

df\$X4 <- NULL \#remove extraneous column

df\$X1<- $(\mathrm{df} \$ \mathrm{X} 1) /\left(\mathrm{cf}^{\wedge} 2\right)$ \#convert area to micrometers, overwrite in dataframe

df\$Length $<-\operatorname{lw}[1$,$] / cf \# convert length to micrometers, add to dataframe$

df\$Width $<-1 w[2$,$] / cf \#convert width to micrometers, add to dataframe$

df\$LWRatio <- df\$Length/df\$Width \#calculate length/width ratio, add to dataframe

names(df) <- c("Area","Circularity","Rectangularity", "Length", "Width", "LWRatio") \#rename columns $\cdots$

' $\{r\}$

\#Add morphometric data to metadata

len $<$ - length(data)

for ( $\mathrm{i}$ in $\mathrm{c}(1$ :length $(\mathrm{df})))\{$

data[len $+\mathrm{i}]<-$ df $[\mathrm{i}]$

\}

\#\#\#Statolith Rugosity

$\cdots\{r\}$

\#Functions for statolith rugosity (internal angle variance) calculation

\#Calculate the angle between two vectors (in radians)

angleCalc $<$ - function(M,N) \{

$\operatorname{abs}(\operatorname{atan} 2(\mathrm{~N}[2], \mathrm{N}[1])-\operatorname{atan} 2(\mathrm{M}[2], \mathrm{M}[1]))$

\} 
\#Check the calculated angle (in degrees) is the internal angle (toward center, so less than 180 degrees) checkAngle $<$ - function(cent, p1, p2, p3, theta) \{

$\mathrm{p} 4<-\mathrm{c}(((\mathrm{p} 1[1]+\mathrm{p} 3[1]) / 2),((\mathrm{p} 1[2]+\mathrm{p} 3[2]) / 2))$ \#calculate point half way between endpoints $(\mathrm{n}$ and $\mathrm{n}+2)$

$\mathrm{d} 1<-\operatorname{sqrt}\left((\mathrm{p} 2[1]-\operatorname{cent}[1])^{\wedge} 2+(\mathrm{p} 2[2]-\operatorname{cent}[2])^{\wedge} 2\right)$ \#distance from centroid to original mid point $(\mathrm{n}+1)$

$\mathrm{d} 2<-\operatorname{sqrt}\left((\mathrm{p} 4[1]-\operatorname{cent}[1])^{\wedge} 2+(\mathrm{p} 4[2]-\operatorname{cent}[2])^{\wedge} 2\right) \#$ distance from centroid to halfway point

if $(\mathrm{d} 1==\mathrm{d} 2)\{\#$ if distances are equal, then its a 180 degree line

theta $<-180.0$

\} else if $(\mathrm{d} 1>\mathrm{d} 2)\{$ \#if mid point $(\mathrm{n}+1)$ farther away than calculated halfway point, then angle is internal

theta $<$ - theta

\} else $\{$ \#if calculated halfway point farther away than mid point $(n+1)$, then angle is external

theta $<-360$-theta \#subtract from 360 degrees to get internal angle

\}

\}

$\cdots\{r\}$

\#Set outline resolution

res $<-150$ \#number of points

statoSam <-_coo_sample(stato_align, res) \#sample outlines with new resolution …

$\cdots\{r\}$

\#Get the position of the centroids of each statolith outline

center $<$ - coo_centpos(stato_align)

$\cdots$

' $\{r\}$

\#Get the position of the centroids of each statolith outline

$\mathrm{s}<-$ statoSam[h]

$\cdots$

$\cdots\{r\}$

\#Calculate internal angle variances

iAngVar <- c () \#empty array for results

for(h in $\mathrm{c}(1$ :length(statoSam $)))\{$

$\mathrm{s}<-$ statoSam[h] \#pull statolith outline

$\mathrm{s}<-\mathrm{s}[[1]]$ \#pull list of outline points

angle $<-\mathrm{c}()$ \#empty array for angles

for (i in c(1:res)) \{

$\mathrm{x} 1<-\mathrm{s}[\mathrm{i}, 1]$ \#pull xpos of $n$th point

y $1<-s[i, 2]$ \#pull ypos of nth point

if $(i<$ res-1) \#for most point along the outline

$\mathrm{x} 2<-\mathrm{s}[(\mathrm{i}+1), 1]$ \#pull xpos of $\mathrm{n}+1$ th point

$\mathrm{x} 3<-\mathrm{s}[(\mathrm{i}+2), 1]$ \#pull xpos of $\mathrm{n}+2$ th point

$\mathrm{y} 2<-\mathrm{s}[(\mathrm{i}+1), 2]$ \#pull ypos of $\mathrm{n}+1$ th point

y3 <- s[(i+2),2] \#pull ypos of $n+2$ th point

\} else if $(i==$ res-1) $\{$ \#but for the penultimate point on the outline

$\mathrm{x} 2<-\mathrm{s}[(\mathrm{i}+1), 1]$ \#pull xpos of $\mathrm{n}+1$ th point

$\mathrm{x} 3<-\mathrm{s}[1,1]$ \#pull xpos of first point on the outline

$\mathrm{y} 2<-\mathrm{s}[(\mathrm{i}+1), 2]$ \#pull ypos of $\mathrm{n}+1$ th point

y3 <- s[1,2] \#pull ypos of first point on the outline

\} else $\{$ \#and for the last point on the outline

$\mathrm{x} 2<-\mathrm{s}[1,1]$ \#pull xpos of first point on the outline

$\mathrm{x} 3<-\mathrm{s}[2,1]$ \#pull xpos of second point on the outline

y $2<-s[1,2]$ \#pull ypos of first point on the outline 


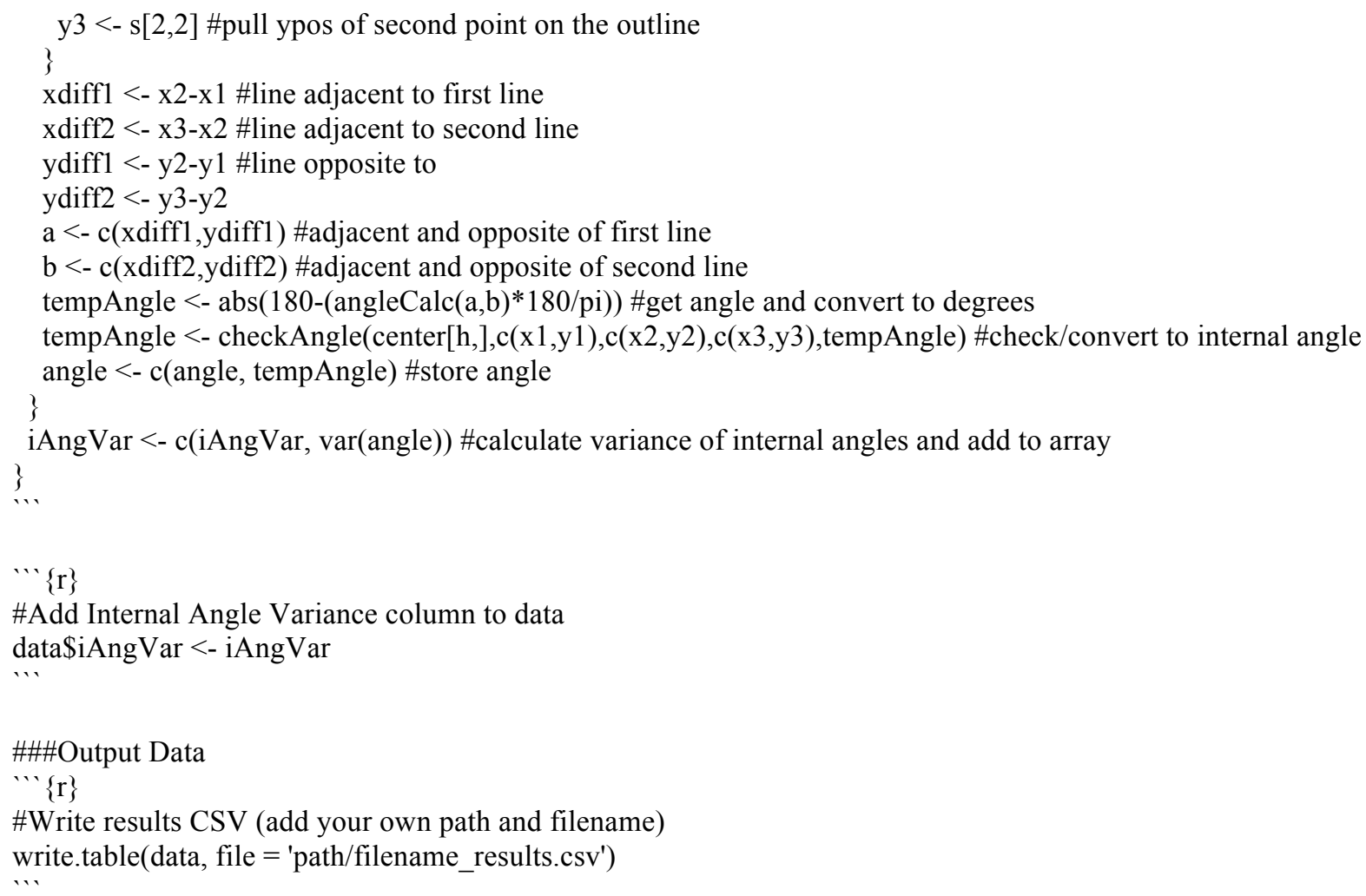




\section{A1.1b Internal angle variance: Resolution assessment}

Two metrics were developed as a potential quantification of statolith edge rugosity:

- Variance of the internal angle of the outline

- Variance of the angle of lines tangent to the outline

A sample set of 50 shapes (Fig. S3), drawn by CZ, was assessed with both metrics at a range of outline resolutions $(50,100,150,250,350$, and 450 points). The five highest and five lowest ranking shapes were assessed for each metric at each resolution. Produced outlines of shapes were also visually compared to their originals to determine maintenance of shape integrity in the resulting outline. Three series of shapes were also assessed separately for their progression within each metric and resolution. These were (shapes within series are listed from most rugose to least):

- Circle-studded squares (CSS)

○ Size 1 [Fig. S3 \#39]

○ Flattened Size 1 [Fig. S3 \#40]

○ Size 2 [Fig. S3 \#38]

○ Size 3 [Fig. S3 \#37]

- $\quad$ Triangle-studded rectangles (TSR)

○ Size 1 [Fig. S3 \#33]

○ Size 2 [Fig. S3 \#34]

○ Size 3 [Fig. S3 \#35]

○ Flattened Size 3 [Fig. S3 36]

- Corals

○ Coral 4 [Fig. S3 \#47]

○ Coral 3 [Fig. S3 \#46]

○ Coral 2 [Fig. S3 \#45]

○ Coral 1 [Fig. S3 \#44]

A subset of the results of these analyses is presented in Tables S5 and S6. The data demonstrated that internal angle variance better represented rugosity (complexity/variability of the outline) while tangent angles represented the "sharpness" or "pointiness" of a shape (e.g. shapes \#12 and \#15 ranked very highly in tangent angle variance despite not being particularly 
rugose; Fig. S3). The 150-point outline resolution maintained shape integrity and produced the best results in both metrics, and the internal angle variance results best fit the concept of rugosity at this resolution, so these were used for the statolith analysis described in the main text. 


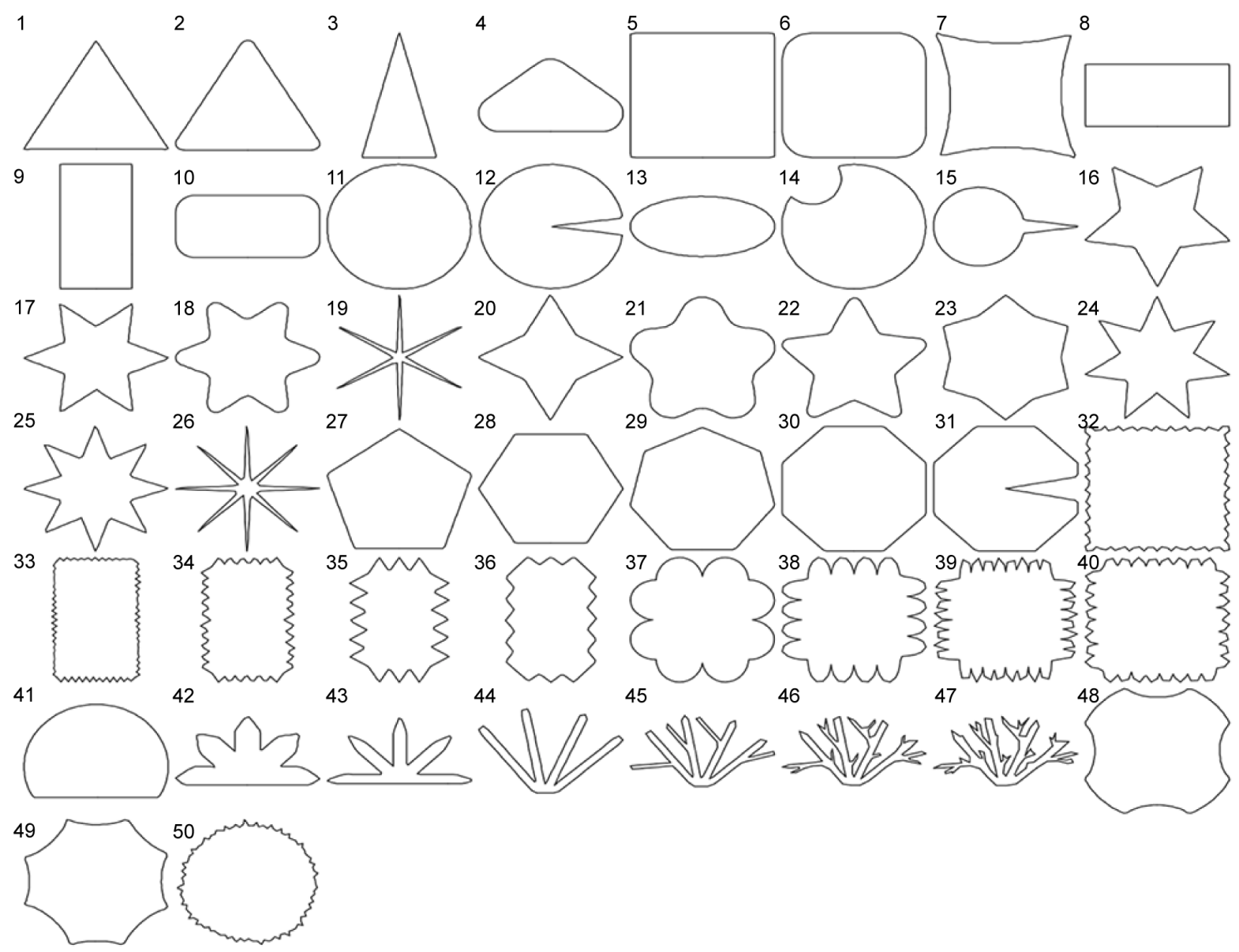

Figure S3. Sample set of shapes for assessment of rugosity metrics and resolution (outlined here at 150 points). Shape outlines presented here were produced using the panel() method of the Momocs R Package. CZ superimposed the numbers for reference 
Table S5. Subset of shape testing data at three of six point resolutions, showing the top 5 and bottom 5 shapes determined by each metric. The datasets were assessed to determine which metric at which resolution best captured and quantified edge variability of the shapes.

\begin{tabular}{|c|c|c|c|c|c|c|c|}
\hline & & Internal Angle Variance & & & Tangent Angle Variance & & \\
\hline Resolution & Rank & Shape Name & \# & Value & Shape Name & \# & Value \\
\hline \multirow{10}{*}{$50 \mathrm{pt}$} & 1 & Coral 4 & 47 & 11040.1 & Circle-studded square, Size 1 & 39 & 0.043 \\
\hline & 2 & Coral 3 & 46 & 9976.5 & Circle-studded square, Flat Size 1 & 40 & 0.020 \\
\hline & 3 & Circle-studded square, Size 4 & 38 & 7915.6 & Coral 4 & 47 & 0.013 \\
\hline & 4 & 8-pointed star & 26 & 6244.1 & Circle-studded square, Size 2 & 38 & 0.010 \\
\hline & 5 & Circle-studded square, Size 1 & 39 & 6117.3 & Coral 3 & 46 & 0.008 \\
\hline & 46 & Cut circle / Brain coral & 41 & 107.3 & Oval & 13 & 1.4E-4 \\
\hline & 47 & Rounded square & 06 & 94.3 & Rounded Square & 06 & 1.3E-4 \\
\hline & 48 & Flat rounded triangle & 04 & 83.9 & Flat rounded triangle & 04 & 1.3E-4 \\
\hline & 49 & Oval & 13 & 66.1 & Rounded rectangle & 10 & $7.5 \mathrm{E}-5$ \\
\hline & 50 & Circle & 11 & 7.1 & Circle & 11 & $3.6 \mathrm{E}-6$ \\
\hline
\end{tabular}

\begin{tabular}{l|l|lll|lll}
\hline \multirow{5}{*}{} & 1 & Circle-studded square, Size 1 & 39 & 7636.1 & Circle-studded square, Size 1 & 39 & 0.013 \\
& 2 & Triangle-studded rectangle, Size 1 & 33 & 6991.3 & Coral 4 & 47 & 0.007 \\
& 3 & Coral 4 & 47 & 6748.1 & Circle-studded square, Flat Size 1 & 40 & 0.006 \\
& 4 & Circle-studded square, Flat Size 1 & 40 & 5581.5 & Coral 3 & 46 & 0.005 \\
& 4 & Spiky circle & 50 & 4517.3 & 8-pointed star & 26 & 0.005 \\
\cline { 2 - 8 } & 46 & Octagon & 30 & 67.5 & Flat rounded triangle & 04 & $1.1 \mathrm{E}-4$ \\
& 47 & Cut circle / Brain coral & 41 & 65.6 & Rounded square & 06 & $1.1 \mathrm{E}-4$ \\
& 48 & Flat rounded triangle & 04 & 27.5 & Cut circle / Brain coral & 41 & $1.0 \mathrm{E}-4$ \\
& 49 & Rounded square & 06 & 25.9 & Rounded rectangle & 10 & $6.7 \mathrm{E}-5$ \\
& 50 & Rounded rectangle & 10 & 22.4 & Circle & 11 & $9.3 \mathrm{E}-7$ \\
\hline
\end{tabular}

\begin{tabular}{|c|c|c|c|c|c|c|c|}
\hline \multirow{10}{*}{$350 \mathrm{pt}$} & 1 & Triangle-studded rectangle, Size 1 & 33 & 3397.7 & Circle-studded square, Size 1 & 39 & 0.006 \\
\hline & 2 & Circle-studded square, Size 1 & 39 & 3126.6 & 8-pointed star & 26 & 0.004 \\
\hline & 3 & Coral 4 & 47 & 2879.2 & 6-pointed star & 19 & 0.004 \\
\hline & 4 & Circle-studded square, Flat Size 1 & 40 & 2270.5 & Coral 3 & 46 & 0.004 \\
\hline & 5 & Spiky circle & 50 & 2159.9 & Coral 4 & 47 & 0.003 \\
\hline & 46 & Tall rectangle & 9 & 64.4 & Octagon & 30 & 7.6E-5 \\
\hline & 47 & Square & 5 & 48.5 & Cut circle / Brain coral & 41 & $6.6 \mathrm{E}-5$ \\
\hline & 48 & Long rectangle & 8 & 48.3 & Rounded rectangle & 10 & $5.9 \mathrm{E}-5$ \\
\hline & 49 & Octagon & 30 & 39.8 & Spiky circle & 50 & $5.5 \mathrm{E}-5$ \\
\hline & 50 & Rounded rectangle & 10 & 34.8 & Circle & 11 & 4.5E-7 \\
\hline
\end{tabular}


Table S6. Subset of shape series data showing results for circle-studded squares (CSS), triangle-studded rectangles (TSR) and coral sets at three outline resolutions. Both metrics produced the correct progression in all three series at 150 points.

\begin{tabular}{|c|c|c|c|c|c|c|c|}
\hline \multirow[b]{2}{*}{ Resolution } & \multirow[b]{2}{*}{ Rank } & \multicolumn{3}{|c|}{ Internal Angle Variance } & \multicolumn{3}{|c|}{ Tangent Angle Variance } \\
\hline & & CSS & TSR & Coral & cSS & TSR & Coral \\
\hline \multirow{4}{*}{$50 \mathrm{pt}$} & 1 & Size 2, \#38 & Size 3, \#35 & 4 & Size 1, \#39 & Size 1, \#33 & 4 \\
\hline & 2 & Size 1, \#39 & Size 2, \#34 & 3 & Flat Size $1, \# 40$ & Size 2, \#34 & 3 \\
\hline & 3 & Flat Size $1, \# 40$ & Flat Size 3, \#36 & 2 & Size 2, \#38 & Size 3, \#35 & 2 \\
\hline & 4 & Size 3, \#37 & Size 1, \#33 & 1 & Size 3, \#37 & Flat Size 3, \#36 & 1 \\
\hline \multirow{4}{*}{$150 \mathrm{pt}$} & 1 & Size 1, \#39 & Size 1 , \#33 & 4 & Size 1, \#39 & Size 1, \#33 & 4 \\
\hline & 2 & Flat Size $1, \# 40$ & Size 2, \#34 & 3 & Flat Size $1, \# 40$ & Size 2, \#34 & 3 \\
\hline & 3 & Size 2, \#38 & Size 3, \#35 & 2 & Size 2, \#38 & Size 3, \#35 & 2 \\
\hline & 4 & Size 3, \#37 & Flat Size 3, \#36 & 1 & Size 3, \#37 & Flat Size 3, \#36 & 1 \\
\hline \multirow{4}{*}{$350 \mathrm{pt}$} & 1 & Size 1, \#39 & Size 1 , \#33 & 4 & Size 1, \#39 & Size 2, \#34 & 3 \\
\hline & 2 & Flat Size $1, \# 40$ & Size 2, \#34 & 3 & Size 2, \#38 & Size 3, \#35 & 4 \\
\hline & 3 & Size 2, \#38 & Size $3, \# 35$ & 2 & Flat Size $1, \# 40$ & Size 1, \#33 & 2 \\
\hline & 4 & Size 3, \#37 & Flat Size 3, \#36 & 1 & Size 3, \#37 & Flat Size 3, \#36 & 1 \\
\hline
\end{tabular}




\section{A1.2 Average pixel intensity variance protocol \& MATLAB code}

The following MATLAB code provides the method for analyzing statolith surface variance (StatoSurfVar) by calculating the average pixel intensity variance across five user-

placed analysis boxes. This code uses the cut PNG statolith images created in Adobe Photoshop (as described in the main text). The path to and the filenames of those images must be saved in a text file in the MATLAB folder entitled 'ssvFiles.txt', with the path as the first line and each individual image filename on its own subsequent line.

The code is run with a String that acts to label the analysis run (e.g. dataset or date of analysis) and uses this to name the output tables. Once the code is running, it will present the first image in the provided list as a MATLAB figure, which you can then click on five times in order to place the pixel variance analysis boxes. The boxes will then appear on the image and you will be prompted to accept them (by clicking 'Yes' or 'No' in the pop-up box) before the program will move on to the next image. The intent is that these boxes are placed haphazardly, but this can result in overlap or capturing surface contaminants within the boxes, so this interface allows you to iteratively place the boxes as desired to fit the sample.

Once you have accepted the squares on the last image in your dataset, the program will output the centroids of all squares of all images to a CSV file entitled with your 'runName' and the variance data to a CSV file entitled 'runName_results'. The final MATLAB Figure will not automatically close, but the program will still have completed. JPEG's of all images with the analysis squares superimposed are output during the program's operation. It is recommended to move all of these to a subfolder named with your 'runName' because they can get substantive with high sample sizes and if a sample is rerun the original JPEG will be overwritten.

The 'runName_results.csv' file has proxy titles of "Variance_\#_\#' (if you can fix this, please do). Otherwise manually rename 'Variance_1_1' as 'Statolith ID', 'Variance_2_1 - 5' as 'Box 1 - 5 Variance', and 'Variance_3_1' as 'Average Variance'. 
function statoSurfVar(runName)

\%\{StatoSurfVar(String) analyzes average pixel variance among five ouser-defined boxes on a statolith image. Per run (named by the input oString runName), StatoSurfvar takes a file path (line 1) and list of image \%file names (remaining lines [separated by $\backslash n]$ ) stored in ssvFiles.txt and oprompts the user to define the centroids of the $100 \mathrm{px}$ x $100 \mathrm{px}$ analysis oboxes by clicking the image five times. The program will then display the oanalysis boxes and prompt the user to confirm them before continuing with othe analysis. StatoSurfVar outputs.jpg images of the input images with othe boxes drawn on top, a .csv containing the centroid positions of the \%five boxes for all input images, and a . Csv with the resultant average \%variances. Note: sID assumes the creator's naming convention and should be \%redefined based on how you ID/how you want to ID your statolith images. $\%$

\%Version 1.1 written by Casey Zakroff (czakroff@whoi.edu) May 22018

oin MATLAB version 2016b on Mac. Code and protocols available at:

\%https://github.com/czakroff/Statoliths

\% \}

\% Read file list

fileID = fopen('ssvFiles.txt','r'); \%open your path/data list

formatspec $={ }^{\prime} \% \mathrm{~s}^{\prime}$;

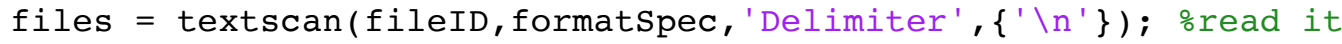

fclose(fileID); \%close it

path $=$ files $\{1\}\{1\}$; ofile path is the first line of ssvFiles.txt

results $=\operatorname{cell}($ length $(f i l e s\{1\})-1,1)$; \%array for the results

\% Image processing loop

for $i=2:($ length $($ files $\{1\}))$ \%from the line after the path to the end

을 Read and display image

filename $=$ files $\{1\}\{i\}$; oread in filename of an individual.png

if isempty(filename) odeals with multiple empty lines at end of list results (length (results)) $=[$ ];

end break;

SID $=$ filename(1:length(filename)-8); \%Alter this based on your ID's im = imread(strcat (path,filename)); \%read in image

imshow(im); \%display image

\%

while(1)

sqrCents $=$ int32(ginput(5)); \%reads 5 user clicks for centroids

sqrpos $=[]$;

for $j=1: 5$ odraw $5100 \mathrm{px}$ squares with input centroids

pos $=[\operatorname{sqrCents}(j,:)-50,100,100]$;

end sqrPos $=\operatorname{cat}(1$, sqrPos, pos $)$;

sqIm = insertshape (im, 'Rectangle', sqrPos); \%draw squares on image imshow(sqIm); oshow image with squares

oprompt user

choice = menu('Accept Analysis Squares?','Yes', 'No');

if choice $=1$ \%end loop if user chooses 'Yes' break;

end

end

imwrite(sqIm, strcat(SID,'_gradSqr.jpg')); ooutput image with squares

\%으일 centroid table

if $i==2$ obuild initial table for centroids of 5 squares

$\mathrm{T}=$ table ( [string(SID); string(SID); string(SID); string(SID); . . string (SID) ], [ $1 ; 2 ; 3 ; 4 ; 5]$, sqrCents);

else oxpand table for each additional image processed 


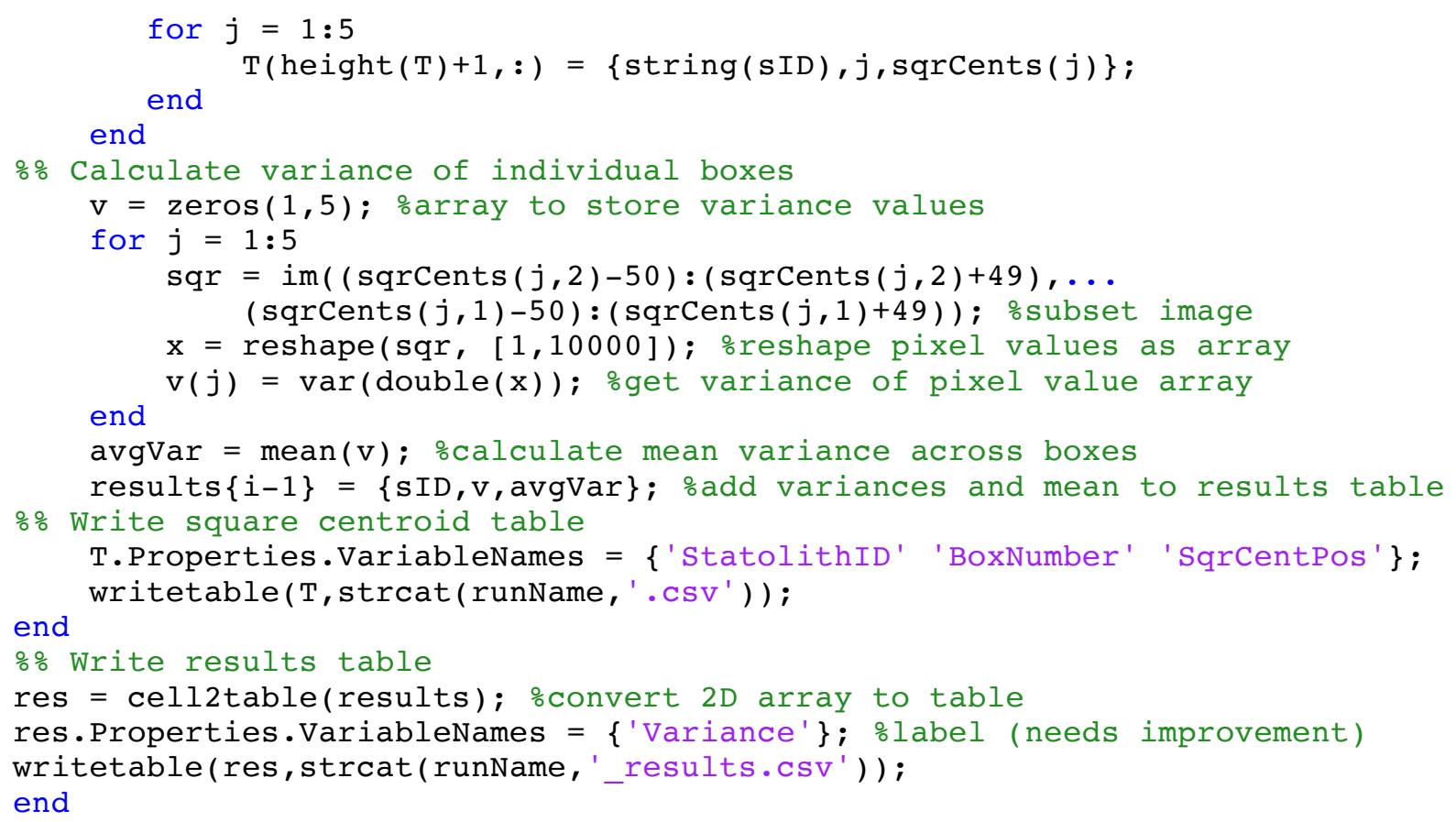




\section{A2. Egg number covariate analyses}

\section{Dorsal Mantle Length}

As stated in the manuscript, egg number shows a weak correlation as a continuous covariate with dorsal mantle length (Fig. S4). Assessing all data, there is only a weak, nonsignificant ( $\mathrm{LR}, P=0.34$ ) trend in the baseline, low $\mathrm{pCO}_{2}$ treatments, but a significant trend across treatments (LR, $P<0.05)$. These trends are skewed in the data by what appears to be an outlier point (although data is very limited related to the population). When this point (Jul 11/ 100 eggs) is removed, the linear relationship between dorsal mantle length and egg number is consistently strong, even when normalized for the shifting baseline state between clutches/trials (Fig. S4).

The slope of the relationship between DML and egg number appears relatively consistent across $\mathrm{CO}_{2}$ treatment bins, suggesting what may be a broadly independent covariate given more data. However, an ANOVA on our data demonstrated significant interactions between egg number and $\mathrm{pCO}_{2}$ in all cases, indicating significantly different slopes (Tables S7, S8). Across trials, egg number was a consistently significant factor in determining paralarvae mantle length and significantly interacted with $\mathrm{pCO}_{2}$ treatment, date, and cup (the last, unsurprising as it functionally is a categorical representation of the same factor in our experimental system; Table S7). In the data compiled across trials, egg number and trial showed the strongest impact on differences in paralarvae mantle length across $\mathrm{pCO}_{2}$ treatments, demonstrating the major influence of season, parentage, and clutch on the state of the paralarvae, although $\mathrm{pCO}_{2}$ was still a significant influence as well (Table S7).

\section{Yolk Volume}

A cursory examination of yolk volume and egg number also suggests a potential relationship, however it is clear in the raw data that this trend is strongly driven by trial differences (Fig. S4). When the data is normalized for shifting baselines between trials, there is still a trend of increasing yolk volume with increasing egg number, but it is non-significant (LR, $P>0.05$ ) and highly variable (Fig. S4). More data would be needed to see if this relationship holds for the population, but would be worthwhile to collect, as it suggests egg capsules with more eggs are broadly more invested in by mothers and may fare better. 
Despite the relative weakness in the correlation between yolk volume and egg number in the regression plots, the statistical models indicate that egg number and $\mathrm{pCO}_{2}$ significantly interact to impact paralarval yolk volume (Table S8). Within trials, the interactions of egg number and $\mathrm{pCO}_{2}$ with both hatching date and cup consistently showed significant impacts on yolk volume (Table S7). When compiled across trials, egg number and $\mathrm{pCO}_{2}$ (as a factor of trial) show independent effects on yolk volume (Table S7).

\section{Embryonic Survival and Hatching Time}

Neither embryonic survival (measured as percent hatched) nor hatching time (measured as time in days to $50 \%$ hatching) showed consistent or significant correlations with egg number. Embryonic survival broadly shows decreases with increasing egg number, but these wash out when differences are normalized for trial differences (Fig. S4). The data across treatments suggests that if a capsule is not entirely successful ( $<95 \%$ hatched) then increasing egg number may exacerbate losses (Fig. S4). As increasing egg number increases the number of oxygen consumers, it may be that embryonic survival is more driven by oxygenation state in our system. Our data may then represent the result of variability in oxygenation due to both the experimental system and the egg capsule.

Hatching time shows decreases in the raw data, with a significant trend across all treatments ( $\mathrm{LR}, P=0.01)$, but this again is clearly driven by trial differences (Fig. S4). When

normalized for shifting baselines between trials, no effect of egg number on hatching time is seen, although the slope of response appears to vary between low (slight increase) and high (decrease) $\mathrm{pCO}_{2}$ treatment bins (Fig. S4). 
Table S7. Type II nested ANOVAs for individual trials and compiled data (normalized by difference from trial mean) of both mantle length and (log-transformed) yolk volume. Egg number is included as an independent continuous covariate. Significant $p$ values ( $\alpha=$ 0.05 ) in bold; ' $<<$ ' indicates a negative exponent of 50 or greater.

\begin{tabular}{|c|c|c|c|c|c|c|c|c|}
\hline \multirow[b]{2}{*}{ Source } & \multicolumn{4}{|c|}{ Mantle Length } & \multicolumn{4}{|c|}{ Yolk Sac Volume } \\
\hline & SS & $\mathrm{df}$ & $F$ & $p$ & SS & $\mathrm{df}$ & $F$ & $p$ \\
\hline \multicolumn{9}{|l|}{ Jul 3} \\
\hline $\mathrm{pCO}_{2}$ & $-8.686 * 10^{-13}$ & 2 & $-3.687^{*} 10^{-11}$ & 1.000 & $8.260^{*} 10^{-15}$ & 2 & $2.765^{*} 10^{-12}$ & 1.000 \\
\hline $\mathrm{pCO}_{2}$ : Date & 3.298 & 15 & 18.663 & $<0.001$ & 0.097 & 15 & 4.336 & $<0.001$ \\
\hline $\mathrm{pCO}_{2}:$ Cup & 5.899 & 6 & 83.462 & $<<0.001$ & 0.016 & 6 & 1.731 & 0.112 \\
\hline Egg Number & 507.5 & 1 & 43,082 & $<<0.001$ & 1.481 & 1 & 991.4 & $<<0.001$ \\
\hline Egg Number: $\mathrm{pCO}_{2}$ & 0.368 & 2 & 15.634 & $<0.001$ & $3.453^{*} 10^{-6}$ & 2 & $1.156^{*} 10^{-3}$ & 0.999 \\
\hline $\begin{array}{l}\text { Egg Number : } \mathrm{pCO}_{2} \text { : } \\
\text { Date }\end{array}$ & 0.286 & 15 & 1.616 & 0.062 & 0.103 & 15 & 4.589 & $<0.001$ \\
\hline $\begin{array}{l}\text { Egg Number : } \mathrm{pCO}_{2} \text { : } \\
\text { Cup }\end{array}$ & 4.450 & 6 & 62.960 & $<<0.001$ & 0.035 & 6 & 3.951 & $<0.001$ \\
\hline Residual & 5.030 & 427 & & & 0.563 & 377 & & \\
\hline \multicolumn{9}{|l|}{ Jul 11} \\
\hline $\mathrm{pCO}_{2}$ & $-9.320 * 10^{-12}$ & 2 & $-4.175^{*} 10^{-10}$ & 1.000 & $-1.169 * 10^{-14}$ & 2 & $-8.596 * 10^{-12}$ & 1.000 \\
\hline $\mathrm{pCO}_{2}:$ Date & 9.271 & 15 & 55.373 & $<<0.001$ & 0.023 & 15 & 2.287 & $<0.001$ \\
\hline $\mathrm{pCO}_{2}:$ Cup & 0.152 & 6 & 2.267 & 0.036 & $-2.268^{*} 10^{-4}$ & 6 & -0.056 & 1.000 \\
\hline Egg Number & 482.6 & 1 & 43,223 & $<<0.001$ & 0.127 & 1 & 187.4 & $<0.001$ \\
\hline Egg Number : $\mathrm{pCO}_{2}$ & 0.011 & 2 & 0.515 & 0.598 & $6.778 * 10^{-3}$ & 2 & 4.984 & $<0.001$ \\
\hline $\begin{array}{l}\text { Egg Number: } \mathrm{pCO}_{2} \text { : } \\
\text { Date }\end{array}$ & 9.572 & 15 & 5.717 & $<<0.001$ & 0.017 & 15 & 1.661 & 0.056 \\
\hline $\begin{array}{l}\text { Egg Number: } \mathrm{pCO}_{2} \text { : } \\
\text { Cup }\end{array}$ & 4.812 & 6 & 7.185 & $<<0.001$ & 0.026 & 6 & 6.463 & $<0.001$ \\
\hline Residual & 5.068 & 454 & & & 0.310 & 456 & & \\
\hline \multicolumn{9}{|l|}{ Aug 7} \\
\hline $\mathrm{pCO}_{2}$ & $-4.320^{*} 10^{-11}$ & 2 & $-1.802 * 10^{-9}$ & 1.000 & $2.316^{*} 10^{-15}$ & 2 & $7.880 * 10^{-12}$ & 1.000 \\
\hline $\mathrm{pCO}_{2}$ : Date & 1.284 & 15 & 7.143 & $<0.001$ & $8.847^{*} 10^{-3}$ & 15 & 4.013 & $<0.001$ \\
\hline $\mathrm{pCO}_{2}:$ Cup & 0.388 & 6 & -5.393 & 1.000 & $-1.135^{*} 10^{-3}$ & 6 & -1.287 & 1.000 \\
\hline Egg Number & 158.8 & 1 & 13,254 & $<<0.001$ & 0.050 & 1 & 342.5 & $<<0.001$ \\
\hline Egg Number : $\mathrm{pCO}_{2}$ & 0.314 & 2 & 13.108 & $<0.001$ & $2.179 * 10^{-4}$ & 2 & 0.741 & 0.477 \\
\hline $\begin{array}{l}\text { Egg Number : } \mathrm{pCO}_{2} \text { : } \\
\text { Date }\end{array}$ & 0.348 & 15 & 1.934 & 0.019 & $3.761 * 10^{-3}$ & 15 & 1.706 & 0.047 \\
\hline $\begin{array}{l}\text { Egg Number : } \mathrm{pCO}_{2} \text { : } \\
\text { Cup }\end{array}$ & 3.838 & 6 & 53.376 & $<0.001$ & $4.667^{*} 10^{-3}$ & 6 & 5.292 & $<0.001$ \\
\hline Residual & 5.441 & 454 & & & 0.065 & 441 & & \\
\hline \multicolumn{9}{|l|}{ Compiled Data } \\
\hline Trial & 0.002 & 2 & 0.065 & 0.937 & 328.1 & 2 & $2.227^{*} 10^{5}$ & $<<0.001$ \\
\hline Trial : $\mathrm{pCO}_{2}$ & 21.08 & 15 & 120.8 & $\ll 0.001$ & 1373 & 15 & $1.243^{*} 10^{5}$ & $<<0.001$ \\
\hline Trial : $\mathrm{pCO}_{2}$ : Date & 425.0 & 306 & 119.3 & $<<0.001$ & -0.433 & 306 & -1.920 & 1.000 \\
\hline Trial : $\mathrm{pCO}_{2}$ : Cup & 0.033 & 36 & 0.078 & 0.999 & $6 * 10^{-6}$ & 36 & $2.345^{*} 10^{-4}$ & 1.000 \\
\hline Egg Number & 0.209 & 1 & 17.98 & $<0.001$ & 32.22 & 1 & 43,739 & $<<0.001$ \\
\hline Egg Number : Trial & 302.3 & 2 & 12,987 & $<<0.001$ & -0.026 & 2 & -17.74 & 1.000 \\
\hline $\begin{array}{l}\text { Egg Number : Trial : } \\
\mathrm{pCO}_{2}\end{array}$ & 67.91 & 15 & 389.0 & $<<0.001$ & $-34,307$ & 15 & $-3.105^{*} 10^{6}$ & 1.000 \\
\hline $\begin{array}{l}\text { Egg Number : Trial : } \\
\mathrm{pCO}_{2} \text { : Date }\end{array}$ & 590.7 & 306 & 165.9 & $<<0.001$ & 0.218 & 306 & 0.969 & 0.325 \\
\hline $\begin{array}{l}\text { Egg Number : Trial : } \\
\mathrm{pCO}_{2}: \text { Cup }\end{array}$ & 92.10 & 36 & 219.8 & $<<0.001$ & 0.026 & 36 & 0.969 & 0.325 \\
\hline Residual & 15.54 & 1335 & & & 0.939 & 1274 & & \\
\hline
\end{tabular}


Table S8. Type II ANOVAs of $\mathrm{pCO}_{2}$, with egg number as an independent covariate, separated by metric and trial. Significant p-values $(<0.05)$ in bold. Yolk volume measurements were log-transformed prior to statistical analysis.

\begin{tabular}{|c|c|c|c|c|c|c|c|c|c|c|}
\hline \multirow[b]{2}{*}{ Source } & \multicolumn{5}{|c|}{ Mantle Length } & \multicolumn{5}{|c|}{ Yolk Sac Volume } \\
\hline & SS & df & $F$ & $p$ & $\Omega^{2}$ & SS & $\mathrm{df}$ & $F$ & $p$ & $\Omega^{2}$ \\
\hline \multicolumn{11}{|l|}{ Jul 3} \\
\hline $\mathrm{pCO}_{2}$ & 0.501 & 2 & 19.008 & $<0.001$ & 0.070 & 0.027 & 2 & 7.466 & $<0.001$ & 0.070 \\
\hline Egg Number & 0.060 & 1 & 4.558 & 0.033 & 0.007 & 0.040 & 1 & 22.087 & $<0.001$ & 0.007 \\
\hline Egg Number: $\mathrm{pCO}_{2}$ & 0.199 & 2 & 7.540 & $<0.001$ & 0.025 & 0.019 & 2 & 5.101 & $<0.01$ & 0.025 \\
\hline Residual & 6.047 & 459 & & & & 0.743 & 406 & & & \\
\hline \multicolumn{11}{|l|}{ Jul 11} \\
\hline $\mathrm{pCO}_{2}$ & 1.108 & 2 & 41.903 & $<0.001$ & 0.140 & 0.008 & 2 & 5.014 & $<0.01$ & 0.016 \\
\hline Egg Number & 0.074 & 1 & 5.563 & 0.019 & 0.008 & 0.001 & 1 & 1.747 & 0.187 & 0.001 \\
\hline Egg Number : $\mathrm{pCO}_{2}$ & 0.115 & 2 & 4.351 & 0.013 & 0.011 & 0.006 & 2 & 3.798 & 0.023 & 0.011 \\
\hline Residual & 6.409 & 485 & & & & 0.367 & 489 & & & \\
\hline \multicolumn{11}{|l|}{ Aug 7} \\
\hline $\mathrm{pCO}_{2}$ & 0.390 & 2 & 13.368 & $<0.001$ & 0.044 & $3.27^{*} 10^{-4}$ & 2 & 0.983 & 0.375 & 0.000 \\
\hline Egg Number & 0.591 & 1 & 40.523 & $<0.001$ & 0.070 & $6.12^{*} 10^{-4}$ & 1 & 3.676 & 0.056 & 0.005 \\
\hline Egg Number: $\mathrm{pCO}_{2}$ & 0.169 & 2 & 5.774 & $<0.01$ & 0.017 & 0.002 & 2 & 7.345 & $<0.001$ & 0.026 \\
\hline Residual & 7.107 & 490 & & & & 0.079 & 474 & & & \\
\hline \multicolumn{11}{|l|}{ Compiled } \\
\hline $\mathrm{pCO}_{2}$ & 2.060 & 5 & 29.702 & $<0.001$ & 0.086 & 0.186 & 5 & 39.483 & $<0.001$ & 0.111 \\
\hline Egg Number & 0.245 & 1 & 17.677 & $<0.001$ & 0.010 & 0.055 & 1 & 58.470 & $<0.001$ & 0.033 \\
\hline Egg Number : $\mathrm{pCO}_{2}$ & 0.772 & 5 & 11.125 & $<0.001$ & 0.031 & 0.099 & 5 & 21.045 & $<0.001$ & 0.058 \\
\hline Residual & 19.93 & 1437 & & & & 1.296 & 1375 & & & \\
\hline
\end{tabular}


Figure S4. Relationships between metrics and number of eggs per capsule (next page). Data are presented as raw data (left two columns) and normalized (right two columns: "Lowest $\mathrm{pCO}_{2}$ " $[550,850,400 \mathrm{ppm}]$ by treatment mean, "All Treatments" by trial mean). Hatching time is presented here as the time (in days) to $50 \%$ hatching calculated from the cumulative hatching curves presented in Fig. 7 of the manuscript. Symbols represent means, shape represent egg clutch lay date/trial $($ circle $=$ Jul 3, square $=$ Jul 11, triangle $=$ Aug 7), color represents binned $\mathrm{pCO}_{2}$ treatments (light gray $=$ low $[550,850,400 \mathrm{ppm}]$, medium gray $=$ mid $[1300,1300,1900$ ppm], dark gray = high [2200 ppm]; bins were used instead of a gradient for simplicity). Yolk volume means are back calculated from logarithmic scale in the raw data, but not for the differences used in the normalized data. Lines represent linear regressions; significant $p$ values $(\alpha=0.05)$ are marked by an asterisk. Black regression line and statistics represent all data points. The gray dashed regression line and statistics in the "Dorsal Mantle Length" row have removed the 'Jul 11/ 100 egg' data point as an outlier 


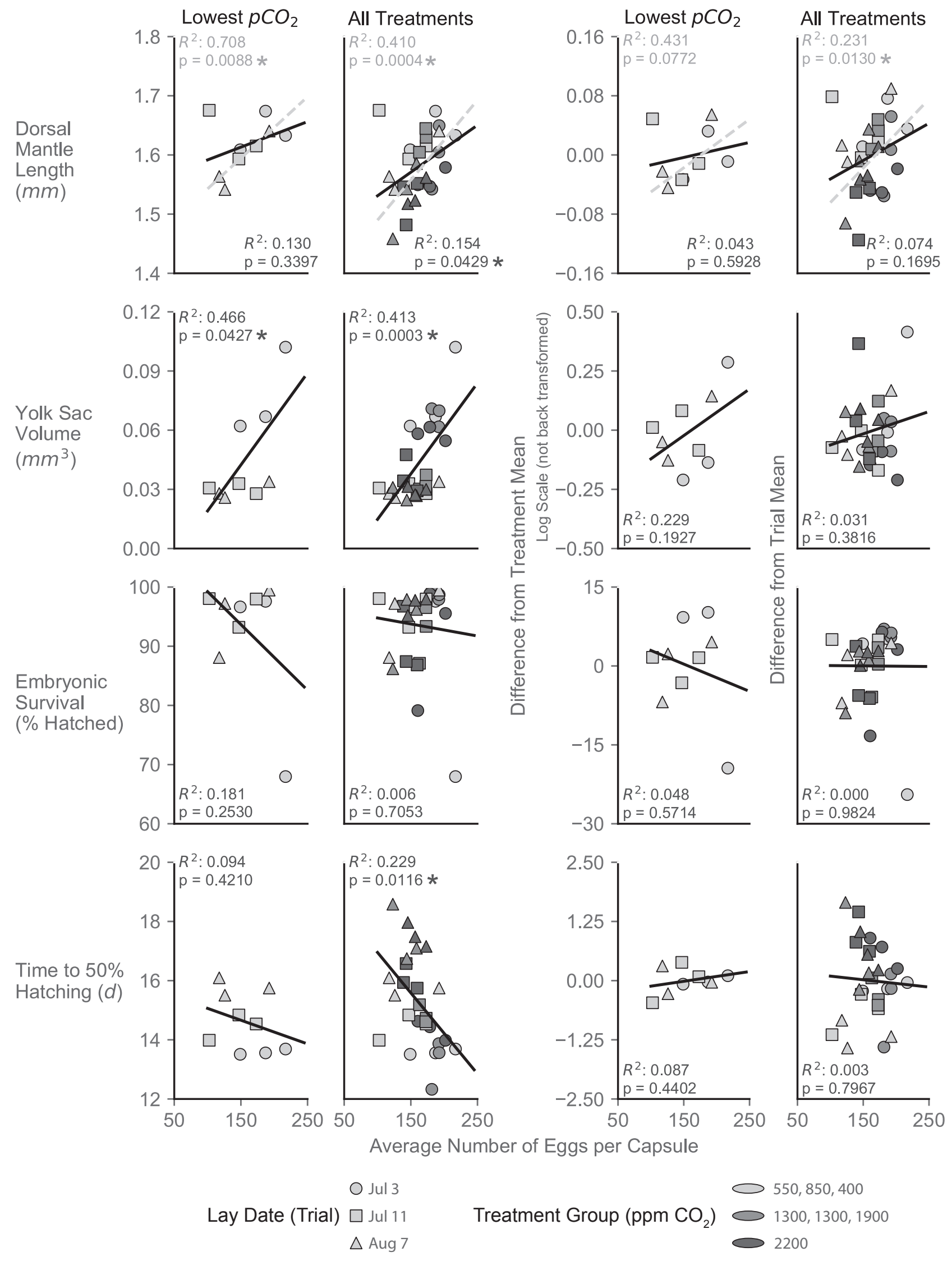




\section{Appendix B}

\section{Chapter 3 Supplementary Materials}

This chapter was originally published as: Zakroff C, Mooney TA, Wirth C (2018) Ocean acidification responses in paralarval squid swimming behavior using a novel 3D tracking system. Hydrobiologia 808:83-106. doi: 10.1007/s10750-017-3342-9. 


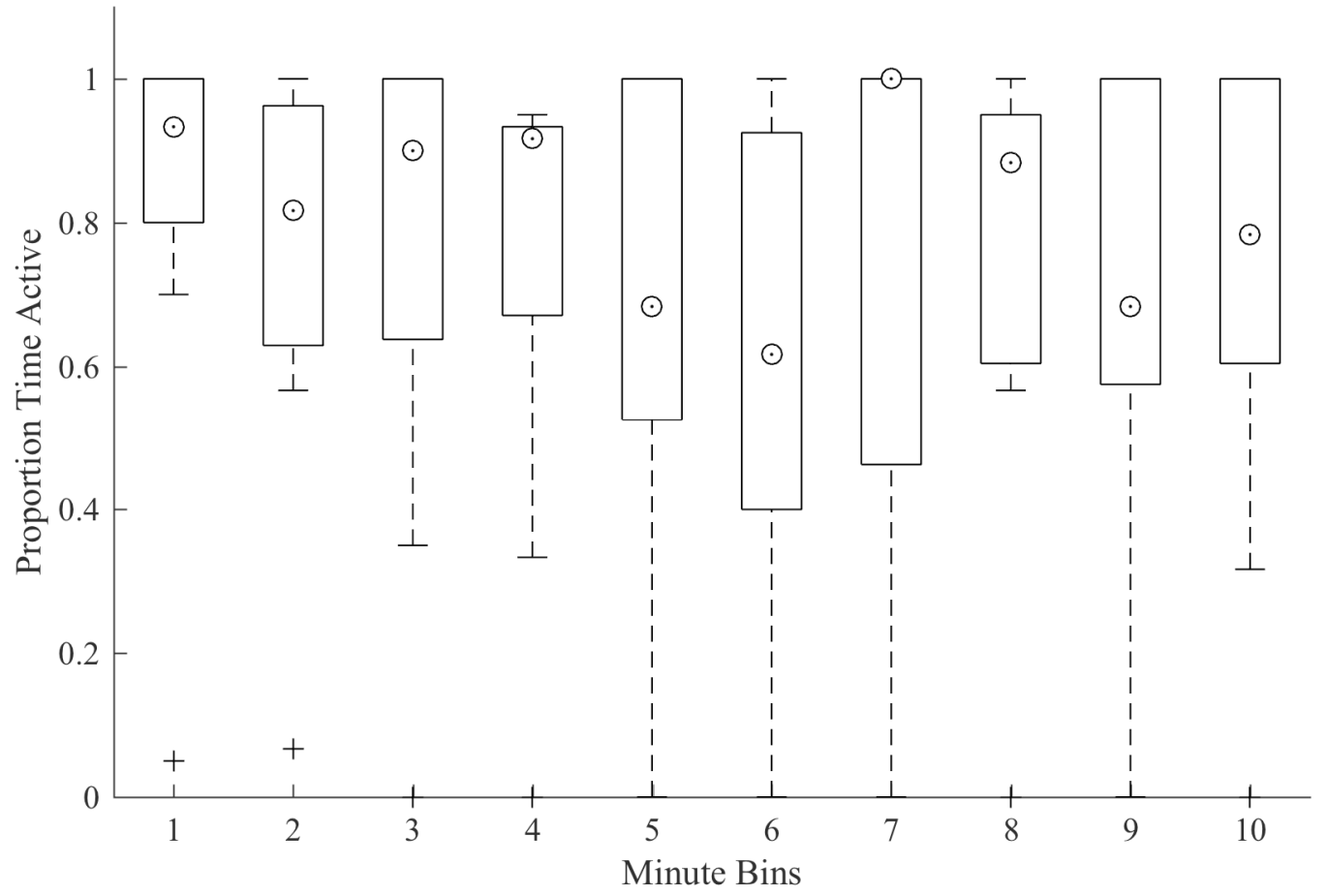

Figure S1 The proportion of individual paralarval $(n=9)$ time spent active in one-minute bins over a 10-minute period in the 3D experimental arena. Paralarvae from all $4 \mathrm{CO}_{2}$ treatments were used and no consistent pattern with $\mathrm{CO}_{2}$ exposure was seen, so all data is compiled here. Dotted circles denote medians and plus signs denote outliers 
A

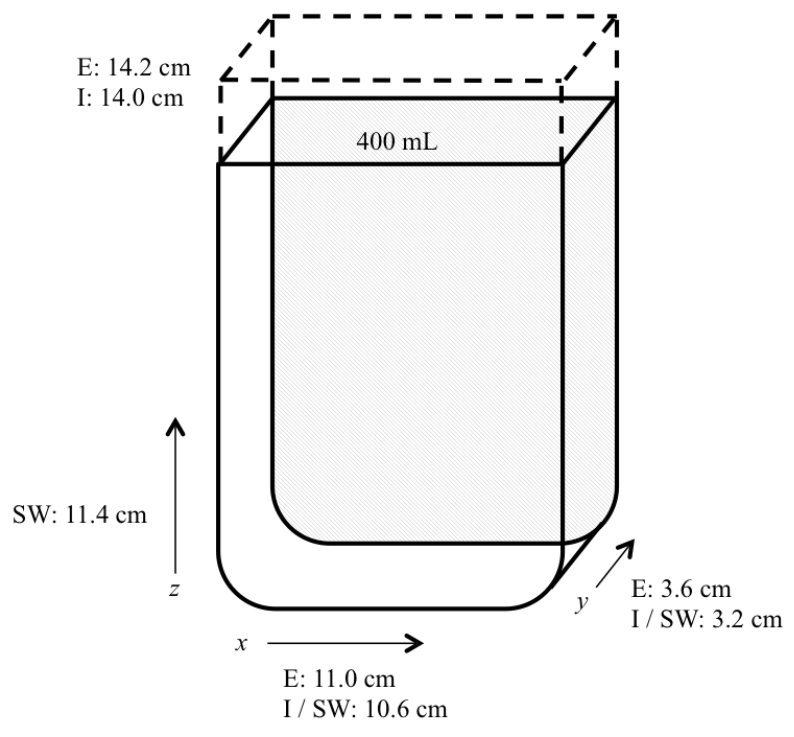

B

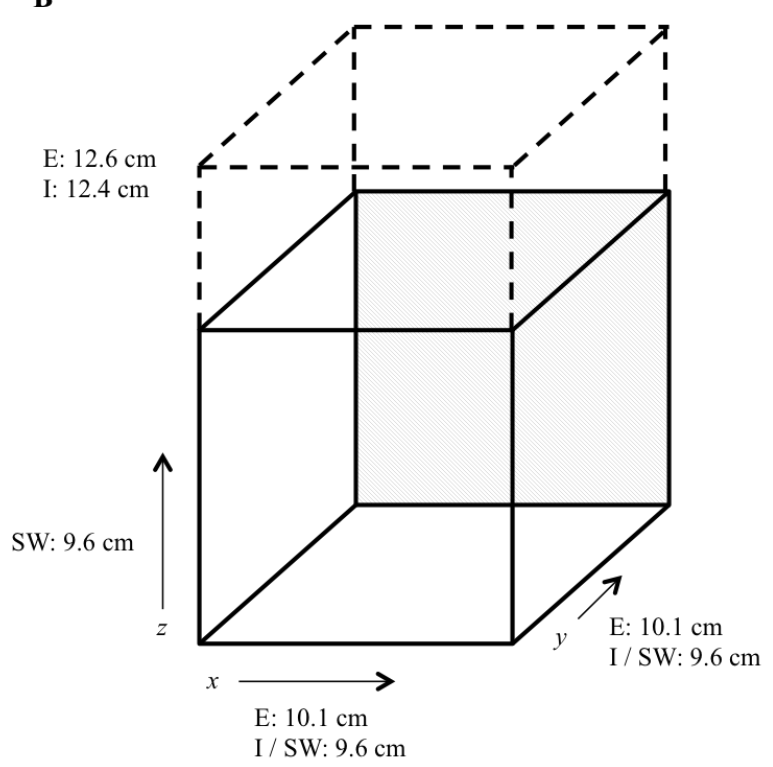

Figure S2 (A) Dimensions and axes of the 2D arena, constructed from a tissue culture flask. 400 $\mathrm{mL}$ of treatment-appropriate seawater was used. (B) Dimensions and axes of the 3D arena constructed from a plastic box. Seawater was added to fill to an internal cube of $9.6 \mathrm{~cm}$. For both schematics, solid lines indicate the seawater space used in the experiments, while dotted lines indicate the additional makeup of the container. Measurements are provided for both external (E) and internal (I) dimensions of the containers, and seawater volume (SW) utilized. The $z$ axis is oriented as it was for tracking, in the bottom, front, left $(0,0,0)$ corner. Note that this was transformed, so that 0 was at the water surface, for manuscript figures depicting depth. Figures are not drawn to scale 


\section{B1. Ocean Acidification and Squid Egg Capsule Culture System}

Experiments took place at the WHOI Environmental Systems Laboratory (ESL), Woods Hole, MA from May - October of 2013, 2014, and 2015. A flow-through ocean acidification (OA) system was equilibrated to different $\mathrm{CO}_{2}$ levels for each trial. Ambient ocean water was pumped in from the ESL intake, passed through the facility's sand-filtration system, and was heated to $20^{\circ} \mathrm{C}$. This temperature reflected peak values reached during the breeding season and resulted in a consistent two-week development period for the ambient paralarvae. The seawater was then passed through a $10 \mu \mathrm{m}$ filter (Hayward FLV Series industrial filter equipped with $10 \mu \mathrm{m}$ felt bag) to remove major particulates and a UV sterilizer (Emperor Aquatics Smart HO UV Sterilizer, Model 025150) to eliminate potentially harmful protozoans. The cleaned water subsequently flowed into the experimental system's header tank and was bubbled with compressed air. Water flowed in a gravity-based system from the header tank and was split among four treatment lines into H-shaped PVC equilibration (EQ) chambers. Each leg of an EQ chamber contained two air stones, which bubbled in the appropriate $\mathrm{CO}_{2}$ mixture. Due to the elevated $\mathrm{CO}_{2}$ concentration of ESL seawater (550 ppm in facility compared to $400 \mathrm{ppm}$ at the pump intake in Vineyard Sound) the ambient treatment line was comprised of three EQ chambers, the first degassing with $\mathrm{N}_{2}$ and the following two reintroducing $\mathrm{O}_{2}$ and $\mathrm{CO}_{2}$ at ambient concentrations. Measurements of dissolved oxygen downstream of the nitrogen system were all above 95\%. Due to a facility malfunction, Trial 3 of 2013 lost oxygenation downstream of the nitrogen degassing and was not used. To reduce the growth of brown algae, which was encouraged by the nitrogen enrichment, lines were cleaned between each trial and a $1 \mu \mathrm{m}$ filter was placed downstream of the EQ chambers on the ambient line.

Compressed air, delivered at 30 psi from an indoor air compressor, was delivered using a six-way manifold to the header tank aeration, ambient EQ chambers' air stones, and three mass flow controllers (Aalborg GFC17, Orangeburg, NY, USA) with a flow rate of $4.51 \mathrm{~min}^{-1}$. Pure $\mathrm{CO}_{2}$ was delivered from a cylinder at 30 psi to three further mass flow controllers (Aalborg GFC17), which were set at various flow rates to produce the desired $\mathrm{CO}_{2}$ concentrations in the gas mixtures. Air and $\mathrm{CO}_{2}$ lines were connected downstream of the mass flow controllers and allowed to mix before being split among the EQ chambers' air stones and experimental aquaria bubbling lines. $\mathrm{CO}_{2}$ mixtures covered a range of values between ambient (400 ppm) and 2200 ppm (Main Text, Table 1). $\mathrm{CO}_{2}$ concentrations for each level were measured before the start of 
each trial on a Qubit Systems $\mathrm{CO}_{2}$ Analyzer (model s151) calibrated with three commercial reference standards $(0,362$, and $1036 \mathrm{ppm})$.

Equilibrated water in each treatment line outflowed into a PVC manifold and entered drip lines connected to the experimental aquaria, consisting of individual 1-liter PET food service containers (Solo Foodservice, Lake Forest, IL). The containers had been seawater soaked for 24 hours and DI-water rinsed in advance to remove any potential chemical residues or toxins. Cups were sealed with fitted lids pierced with two holes for the gas and equilibrated water lines. Each cup had a small $(2 \times 4 \mathrm{~cm})$, screened $(5 \mu \mathrm{m}$ mesh) hole to permit water outflow while retaining paralarvae. The bubbler of the gas line was placed under the screen to create flow and prevent paralarvae from sticking to the screening. Water outflowed from the cups into a common water bath maintained at a temperature of $20^{\circ} \mathrm{C}$ via aquarium heaters (JÄGER 3603, EHEIM GmbH and Co., Deizisau, DE) and chillers (Oceanic Aquarium Chiller 1/10hp, Oceanic Systems, Walnut Creek, CA, USA).

In 2013, each trial consisted of three $\mathrm{CO}_{2}$ treatments, each with three experimental cups and one organism-free control cup, totaling twelve cups per water bath. In 2014 and 2015, each trial consisted of four $\mathrm{CO}_{2}$ treatments, totaling sixteen cups per water bath. The system was run for several days prior to a trial to ensure stable water and gas levels. Flow rates to the cups were set at a slow drip, approximately 20 liters day $^{-1}$, which prevented waste accumulation. The flow rate also allowed sufficient time for bubbled gas to equilibrate with water in the EQ chambers. The ESL room containing the experimental set up was kept on a 14:10 hour light:dark photoperiod, reflecting the average photoperiod of the region, using ceiling mounted fluorescent lighting. Water bath temperature and ambient light levels were monitored using an Onset HOBO data logger (pendant model UA-004-64), one in each water bath, with recordings taken every 15 minutes. Temperatures were $20.49 \pm 0.69{ }^{\circ} \mathrm{C}, 20.36 \pm 1.80{ }^{\circ} \mathrm{C}$, and $20.01 \pm 1.00{ }^{\circ} \mathrm{C}($ mean $\pm \mathrm{SE}$ ) in water bath 1 and $20.26 \pm 0.49{ }^{\circ} \mathrm{C}, 20.18 \pm 1.74{ }^{\circ} \mathrm{C}$, and $20.51 \pm 1.91{ }^{\circ} \mathrm{C}$ in water bath 2 in 2013, 2014, and 2015, respectively.

Once squid were brought to the ESL holding tanks, females began laying eggs within two to three days, producing small egg mats typically found at the bottom of the tank or attached to the air hose. The morning an egg cluster was found, it was removed to a holding container and examined for quality. Individual egg capsules, each containing between 50-200 eggs, were then 
randomly selected and randomly assigned to each experimental cup, with two egg capsules per cup (18 egg capsules per trial for 2013, 24 for 2014 and 2015), marking the start of a trial.

During a trial, $\mathrm{pH}$ measurements were taken for samples from each cup every three days using a pH meter (Orion Star ${ }^{\mathrm{TM}}$ A329, Thermo Fisher Scientific Inc., Waltham, MA, USA) in order to monitor $\mathrm{CO}_{2}$ level stability. The fourth cup from each $\mathrm{CO}_{2}$ level contained no eggs sacs and was used as a control for water quality and carbonate chemistry measurements. Spectrometric $\mathrm{pH}$ measurements were taken with a spectrophotometer using methods adapted from Clayton and Byrne (1993) and Dickson et al. (2007) Salinity was measured using a salinity probe (Orion Star ${ }^{\mathrm{TM}}$ A329, Thermo Fisher Scientific Inc., Waltham, MA, USA) in parallel to spectrophotometric $\mathrm{pH}$ readings. Total alkalinity (AT) samples were taken in $20 \mathrm{~mL}$ acid-washed, glass scintillation vials and poisoned with $10 \mu \mathrm{L}$ saturated mercuric chloride $(\mathrm{HgCl} 2)$. Alkalinity samples were analyzed post-trial using an automated small volume titrator (Titrando 808, Metrohm AG, Herisau, $\mathrm{CH}$ ) programmed to run Gran titrations of $1 \mathrm{~mL}$ subsamples. Samples were run in duplicate and calibrated against standards of ESL water of known alkalinity. For duplicates with a difference of $4 \mu \mathrm{mol} \mathrm{kg}{ }^{-1}$ seawater (SW) or greater, samples were rerun and an average of the four values was taken. Carbonate chemistry metrics (temperature, salinity, $\mathrm{pH}$, and alkalinity) were input into CO2SYS, using dissociation constants from Mehrbach (1973) and sulfate constants from Dickson (1990), to calculate $\mathrm{pCO}_{2}$ and aragonite saturation state $\left(\Omega_{\text {arag }}\right)$ for each treatment of each trial. A baseline measurement of all cups was taken prior to the initiation of a trial, followed by weekly readings of only the control cups once a trial had begun. Measured seawater $\mathrm{CO}_{2}$ concentrations fell within reasonable range of the equilibrations desired from the gas concentrations (Main Text, Table 1).

\section{B2. Recording 3D Swimming Behavior Videos}

\section{B2.1 3D Swimming Behavior Recording Protocol}

1. Before sampling your organism, make sure the setup is complete as follows (Note: all cameras and the organism aquaria/chamber were placed within a covered photography light box to block ambient light):

a) Fill the chamber with seawater appropriate to the treatment you are testing. The water level needed to make a perfect cube $(9.6 \mathrm{~cm}$ for this chamber) is marked with a small black line. Remove any bubbles by scraping with a ruler. 
b) Secure the top camera to a sturdy frame above where your chamber will be placed.

c) Place the chamber beneath the top camera. Adjust the top camera zoom so the entire chamber is clearly visible within the image frame.

d) Center the chamber in the camera view. This can be done by measuring the distance between the sides of the chamber and the edge of the image frame for each side and making this distance on both sides equal.

e) Ensure the camera is viewing the center of the chamber straight on and is not skewed to any angle. This can be done by measuring the length (in the camera image) from the top (front) edge of each side of the chamber to its bottom (back) edge and adjusting the position of the camera and chamber to make these distances equal.

f) Place the side camera on a raised block in front of the chamber, such that the camera points to the center of the chamber. Adjust the zoom so the entire chamber is clearly visible. Center the camera on the chamber and adjust skew, as above. Connect the cameras to viewing monitors outside your light box.

g) Place one light on either side of the chamber (perpendicular, non-biased lighting is necessary for photopositive organisms only). Set lighting so your organism is clearly visible in both camera images and reflections from your chamber are reduced as much as possible.

2. Select one individual and place it into the center of the chamber.

3. Close the cover of the light box. Allow organism to acclimate to chamber (one minute was suitable for squid paralarvae).

4. Start timer and record for desired length (two minutes was suitable for squid paralarvae).

5. Before stopping the recording, use a light, laser, or other visual cue so that both videos can easily by synced for 3D analysis.

6. Stop the recording. Remove organism. Repeat steps 3-7 for as many individuals as you have per treatment.

7. Record file number for top and side camera and relevant experimental data/individual identification on a data sheet, along with any notes on video quality (good swimming, corner, etc). 
8. Note: Remember to change water in filming chamber as needed as you progress through your treatments. The camera adjustments outlined in step 1 must be repeated/checked each time the organism chamber is removed or replaced.

9. Upload video files to an external hard drive (recommended due to size and number of videos). Save files in a dated folder, and within that, either a "top" or "side" subfolder.

10. Double check to ensure your recordings are saved and backed up before reformatting the memory cards for the next recording session.

\section{B3. Analysis of 3D Swimming Behavior Data}

\section{B3.1 Organism Tracking Protocol}

1. Drag your video into Tracker (available at OpenSourcePhysics.org). It is best to analyze videos one minute at a time because Tracker can slow down and can run out of memory (amount of memory available to Tracker can be adjusted by clicking memory in use $\rightarrow$ Set memory size...). Save as you go. If the memory allotment gets overloaded the program can crash. At a higher memory allotment, a full two-minute video can be analyzed in one Tracker run. In order to clear the memory cache, it is easiest to close and reopen Tracker for each video analyzed.

2. In the top right corner, click the "refresh" button then deselect Auto-refresh. This prevents available memory from filling up as you track, which slows down the process.

3. You may need to correct for stretching due to Tracker's pixel size, which may differ from those used your camera. Click Video $\rightarrow$ Filters $\rightarrow$ New $\rightarrow$ Resize, then adjust. For the Sony HD Handycams used in the squid experiment the adjustment was:

\begin{tabular}{|c|c|c|}
\hline & Width & Height \\
\hline Input & $\mathbf{1 4 4 0}$ & $\mathbf{1 0 8 0}$ \\
\hline Output & $\mathbf{1 4 4 0}$ & $\mathbf{8 0 0}$ \\
\hline
\end{tabular}

4. Adjust the axis to the desired position by clicking the magenta axis button (show or hide the coordinate axes) on the tool bar. From the side view, the origin $(0,0)$ point should be in the top left corner of the chamber with the y-axis aligned with the inside of the left edge of the chamber and the x-axis aligned with the water level. You can tilt the axes, if needed, by clicking and dragging them. The axes for the top view depend on how your camera is oriented in relation to chamber. The origin for all the data should be the top, 
left, front corner of the chamber. Our top camera was placed such that it viewed the chamber with the front edge at the top of the image. Thus, we placed the origin for tracking top videos at the top right corner of the chamber with the y-axis aligned with the inside right edge of the chamber and the $\mathrm{x}$-axis aligned with the inside top edge. This produced negative values for both $\mathrm{x}$ and $\mathrm{y}$ data that had to be later reoriented (multiply by -1) to the correct reference frame.

5. Adjust the scale by pressing the blue Show, hide, or create calibration tools on the toolbar. Click on new $\rightarrow$ calibration tape. A blue, double-headed arrow will appear on screen. Drag the ends of the calibration tape across a known distance, typically the length of your chamber ( $9.6 \mathrm{~cm}$ between the inside edges of the chamber). You can zoom in to be as accurate as possible placing the ends of the tape. Make sure the angle of the tape to the $\mathrm{x}$-axis is 0 . Double click on the box with the blue number and change it to your known length (9.6 in our case) once adjusted perfectly.

6. Frame selection:

a. Calculate the total number of frames analyzed for 1 minute of video. For our cameras this was 1799 , since the camera filmed at 29.97 frames per second.

b. To calculate the frames you would like to analyze, find the frame where the laser pointer first appears signaling the end of the recording and use the frame before as the last. Subtract your desired number of frames -1 (1798, in our case) from the last frame to get the first frame (the frame range is inclusive, hence we subtracted 1798 not 1799).

7. To apply the desired frame range, click on the Clip Settings button on the toolbar. Enter the frame numbers in the window that appears. You can tell the range has been applied because the small black arrows beneath the video scanning bar at the bottom of the screen will have moved to enclose the desired range.

8. To start a new track, click Create a new track $\rightarrow$ Point Mass. Click on the mass a button in the Track Control window and click the Autotracker... button, which will open the Autotracker window.

9. Autotracker: (refer to Help $\rightarrow$ Tracker Help $\rightarrow$ Autotracker for further explanation)

a. Autotracker works best when the organism is a well-lit white dot and against a black background. 
b. To start, shift + control + click on the animal to be tracked. The numbered point is the target; its position is what will be marked in the data column. The circle selects the template image autotracker will search for, it will appear in the autotracker window. It can be manually resized and moved in relation to the target, but the target will stay wherever you have placed it in relation to the template image while tracking (should be inside the circle).

c. The dashed rectangle is the search area, in which autotracker will search for the template image in the next frame. Depending on how fast/far per frame the animal is moving, make this as small as possible.

d. Press the search button for autotracker to automatically check each frame for the image.

e. Watch Autotracker for the entire time! If it is no longer following the animal, push the stop button and backtrack to the frame where the target lost the animal.

f. To create a new template image, delete the current point and shift + control + click on the animal again to create a new key frame. If you do this without deleting the current point you will just create a new template image, the target will not be moved and will place data points incorrectly. Always make sure the target is not placing data points outside the template image.

g. You can also try dragging the search area box back over the organism and then clicking search.

h. If tracker is having a hard time, you can manually track by repeatedly using shift + click to place the target on the animal frame-by-frame until autotracker can be used again.

i. Use the evolution rate to tell autotracker how much the template image will change frame-to-frame; usually it can be lower than default. Use the automark to set the minimum match score needed to mark points. Lower numbers result in more false-matches but fewer stoppages.

10. Autotracker data:

a. Adjust the frame range in Clip Settings to include the entire tracked time, including first and last frames. Select your point mass (usually mass A) from the 
Plot or Table dropdown menu, and then click the Refresh data and views button on the right side of the toolbar.

b. Copy and paste the table data into an Excel document.

c. Autotracker occasionally skips frames, so it is necessary to check for missing data points. In Excel, select all the data and then use the Go To function (Fn+F5 on PC), then click Special and check Blanks. This highlights all blank cells. You can then change fill color to red to be more visible, and then scroll through to find missing data. Then, go back into the Tracker file and find the skipped frame, shift-click to manually mark the position of the organism and copy the needed data back into Excel.

11. Save the tracker file and the Excel file of your data.

12. In Tracker, use a calibration tape (Show, hide, or create calibration tools $\rightarrow$ new $\rightarrow$ calibration tape) to measure the length of the back of the chamber for $q_{b s}$ or $q_{b t}$ (See list of model variables below). These values are the same throughout a certain placement and orientation of the cameras and chamber, so only need to be measured once for each block of recording (i.e. if the cameras or chamber get moved/readjusted, then measure these values again).

\section{B3.2 3D Data Analysis Protocol}

1. Compile the side and top data for each individual into a single Excel file with the columns: time (s), xs, zs, xt, yt (time can be converted to seconds from frame number, $\mathrm{xs}$ and $\mathrm{zs}$ are the $\mathrm{x}$ and $\mathrm{y}$ data from the side view while $\mathrm{xt}$ an $\mathrm{yt}$ are the $\mathrm{x}$ and $\mathrm{y}$ data from the top view, respectively). Ensure that your data is oriented to an origin $(0,0,0)$ at the top, left, front corner of the chamber (particularly your top view data).

2. Open Matlab and run the Behavior3DCorrection code (below). Enter your Excel file name, sheet name, data range, $q_{b s}$ and $q_{b t}$ for the video you are analyzing. The code usually takes several minutes (5 - 10) to run for two minutes of data. This code outputs your corrected time, $\mathrm{x}, \mathrm{y}, \mathrm{z}$ position data in a new .xls or .csv file and automatically calls Behavior3DAnalysis to calculate metrics of movement.

3. Behavior3DAnalysis calculates a number of 3D movement metrics and prints the summary results of this data to a results text file as well as full data to individual .xls or 
.csv files. It also produces several preliminary plots of the individual organism's movement to aid in visualization and further analysis. 


\section{B3.3 List of Model Variables}

\section{List of Model Variables}

$q_{b s} \quad$ length of back plane of chamber in side video image

$q_{b t} \quad$ length of back plane of chamber in top video image

$q_{i s} \quad$ hypotenuse formed between front plane and side view image plane

$q_{i t} \quad$ hypotenuse formed between front plane and top view image plane

$Q \quad$ length of cubic chamber side

$Q_{s} \quad$ length of image plane of organism in side view

$Q_{t} \quad$ length of image plane of organism in top view

$x$

true $\mathrm{x}$ position of organism

$x_{i s} \quad \mathrm{x}$ position in side view image plane

$x_{i t}$

$\mathrm{x}$ position in top view image plane

$x_{s}$

measured $\mathrm{x}$ position in side view tracking

$x_{t}$

measured $\mathrm{x}$ position in top view tracking

y

true y position of organism

$y_{i t}$

y position in organism's top view image plane

$y_{t}$

measured y position in top view tracking

$z$

true $\mathrm{z}$ position of organism

$z_{i s}$

$\mathrm{z}$ position in side view image plane

$z_{S}$

measured $\mathrm{z}$ position in side view tracking 


\section{B3.4 3D Behavior Data Correction MATLAB code}

function Behavior3DCorrection

$\%\{$ Behavior3DCorrection is designed to merge and correct $\mathrm{x}, \mathrm{z}$ (side view) and $\% x, y$ (top view) tracking data derived from video of an organism (a squid oparalarvae) swimming in a cubic arena taken with two perpendicular cameras. \%This code assumes the data is stored in a single Excel spreadsheet where othe columns are: time (s), xs, zs, $x t, y t$. These known data are input into oan overconstrained system of equations and assessed using least sum of osquares to approximate the true $x, y$, and $z$ values with the most minimal oerror. This code accompanies the publication: Zakroff, C. Mooney, TA, oWirth, C. Ocean Acidification Responses in Paralarval Squid Swimming \%Behavior Using a Novel 3D Tracking System. (2017). Hydrobiologia Vol: Pages. DOI :

\%

\&Version 1.5 written by Casey Zakroff (czakroffewhoi.edu) May 112017

oin MATLAB version $2016 \mathrm{~b}$ on Mac. Code and protocols available at:

ohttps://github.com/czakroff/3D-Swimming-Behavior

$\circ\}$

¿User Input of Excel Data Sheet

filename $=$ input('\nEnter the name of the Excel file: ', 's');

sheet $=$ input ('Enter the name of the Excel sheet: ', 's');

dataRange = input('Enter the range of the data cells, i.e. "A5:E3602":', 's'); $\frac{0}{T h i s}$ data range works for 2 minute videos (3598 frames)

pLcode = strcat (filename,'_', sheet); paralarvae ( $\mathrm{pL})$

oRead in $t, x s, z s, x t, y t$

rawData $=$ xlsread (filename, sheet, dataRange);

oEstablish known values of from your arena

$\mathrm{Q}=9.6 ; \% \mathrm{Q}$ is the length of the side of your cubic arena (in $\mathrm{cm}$ )

oRead in Qbs - the length of the back of the arena in the side video image

Qbs = input('Enter Qbs value: ');

oRead in Qbt - the length of the back of the arena in the top video image

Qbt = input('Enter Qbt value: ');

oCalculate the hypoteneuse of the right triangle between $Q$ and $Q$ back for both the side and top.

$\mathrm{qbs}=(\mathrm{Q}-\mathrm{Qbs}) / 2 * \operatorname{sqrt}(2) ;$

qbt $=(Q-Q b t) / 2 * \operatorname{sqrt}(2)$;

oset up output data array and assign time to first column

correctedData $=\operatorname{zeros}(\operatorname{size}($ rawData, 1$), 4)$;

correctedData $(:, 1)=\operatorname{rawData}(:, 1)$;

oAssign xs, zs, $x t$, and yt

$\mathrm{xs}=\operatorname{rawData}(1: \operatorname{size}(\operatorname{rawData}, 1), 2) ;$

$\mathrm{zs}=\operatorname{rawData}(1: \operatorname{size}(\operatorname{rawData}, 1), 3) ;$

$\mathrm{xt}=\operatorname{rawData}(1: \operatorname{size}(\operatorname{rawData}, 1), 4)$;

yt $=\operatorname{rawData}(1: \operatorname{size}(\operatorname{rawData}, 1), 5)$;

oAssign limits and options for fmincon

$A=\operatorname{ones}(1,11) * 0.001$;

$\mathrm{B}=\operatorname{ones}(1,11) * Q$; 


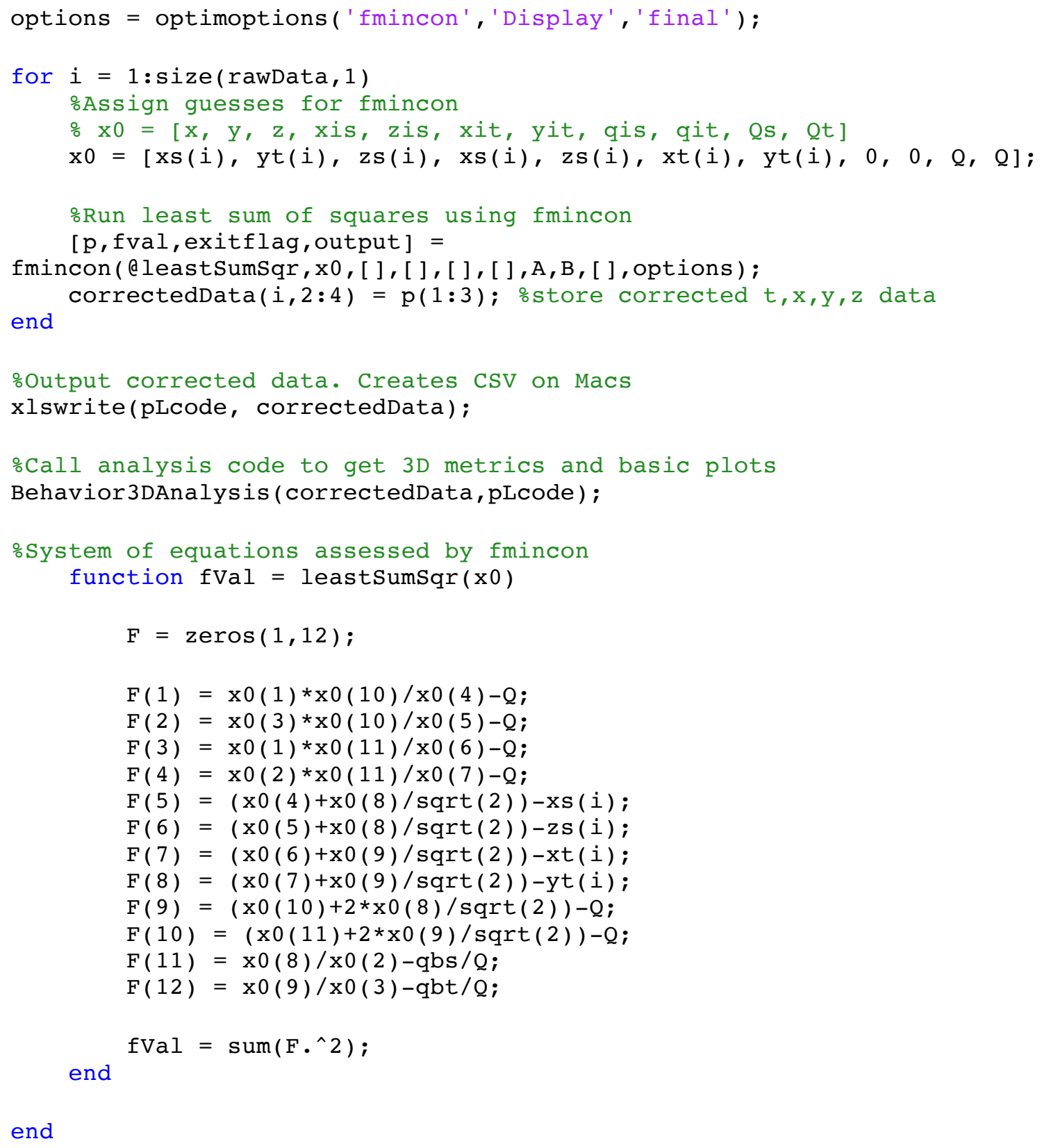

end 


\section{B3.5 3D Behavior Analysis MATLAB code}

\section{function Behavior3DAnalysis(data, filename)}

$\circ\{$ Behavior3DAnalysis takes the corrected $\mathrm{x}, \mathrm{y}, \mathrm{z}$ positional data from ○Behavior3DCorrection and calculates total distance traveled, average, opeak, min, max, and median of 3D, horizontal, and vertical velocity, 3D ovolume covered, 2D turning angles, and path tortuosity (a ratio of the odistance transitited between two points and the true distance between those opoints) of an individual swimming organism. These data are output to a \%'results' text file and to an Excel file named with the individual oorganism ID code. A 3D plot of the organism's swimming track, a plot of othe tortuosity of that path, and a 3D polygon of the volume covered by the oorganism are produced and output as tiff files. A histogram of the oorganism's turning angles over the entire track is output as a png. oThis code accompanies the publication: Zakroff, C. Mooney, TA, \%Wirth, C. Ocean Acidification Responses in Paralarval Squid Swimming \%Behavior Using a Novel 3D Tracking System. (2017). Hydrobiologia. Vol: Pages.

○DOI :

$\%$

\%Version 1.5 written by Casey Zakroff (czakroffewhoi.edu) May 112017 ○in MATLAB version $2016 \mathrm{~b}$ on Mac. Code and protocols available at: ohttps://github.com/czakroff/3D-Swimming-Behavior $\circ\}$

oset variables to set sampling resolution for tortuosity and turning angle res $=30$; $\%$ Sampling resolution (value is in frames; $30 \mathrm{fps}=1 \mathrm{~s}$ segments) numpts $=$ floor $($ size $($ data, 1$) /$ res $) ;$ oNumber of points sampled from the data

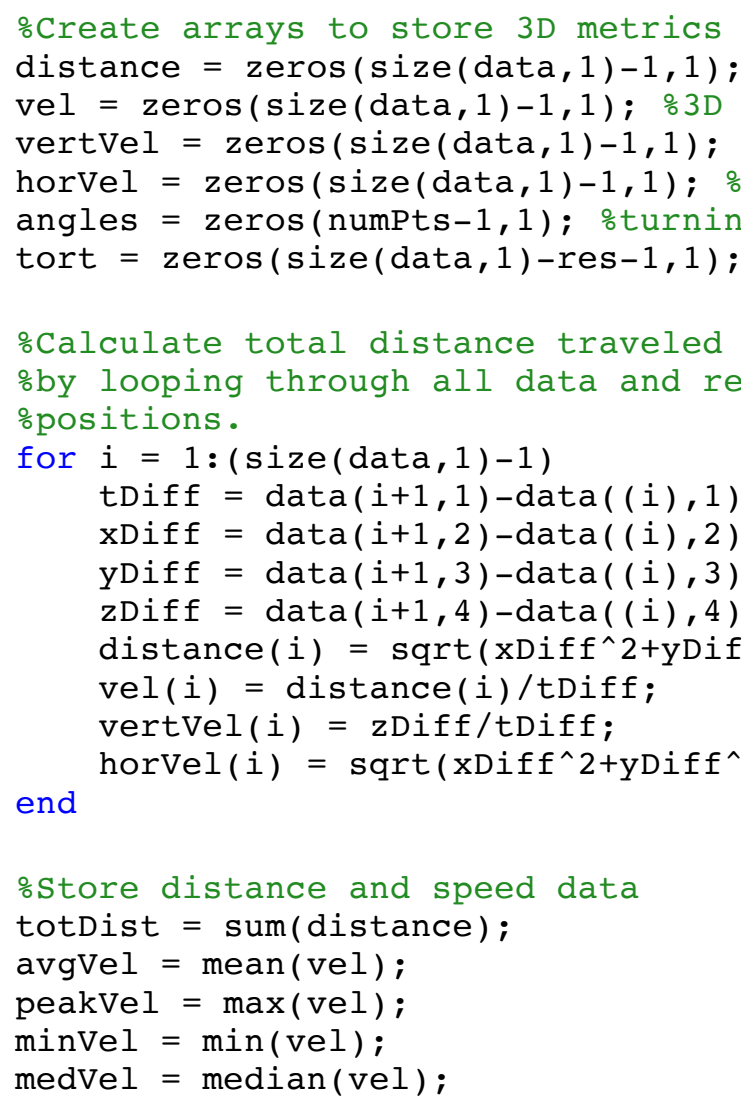




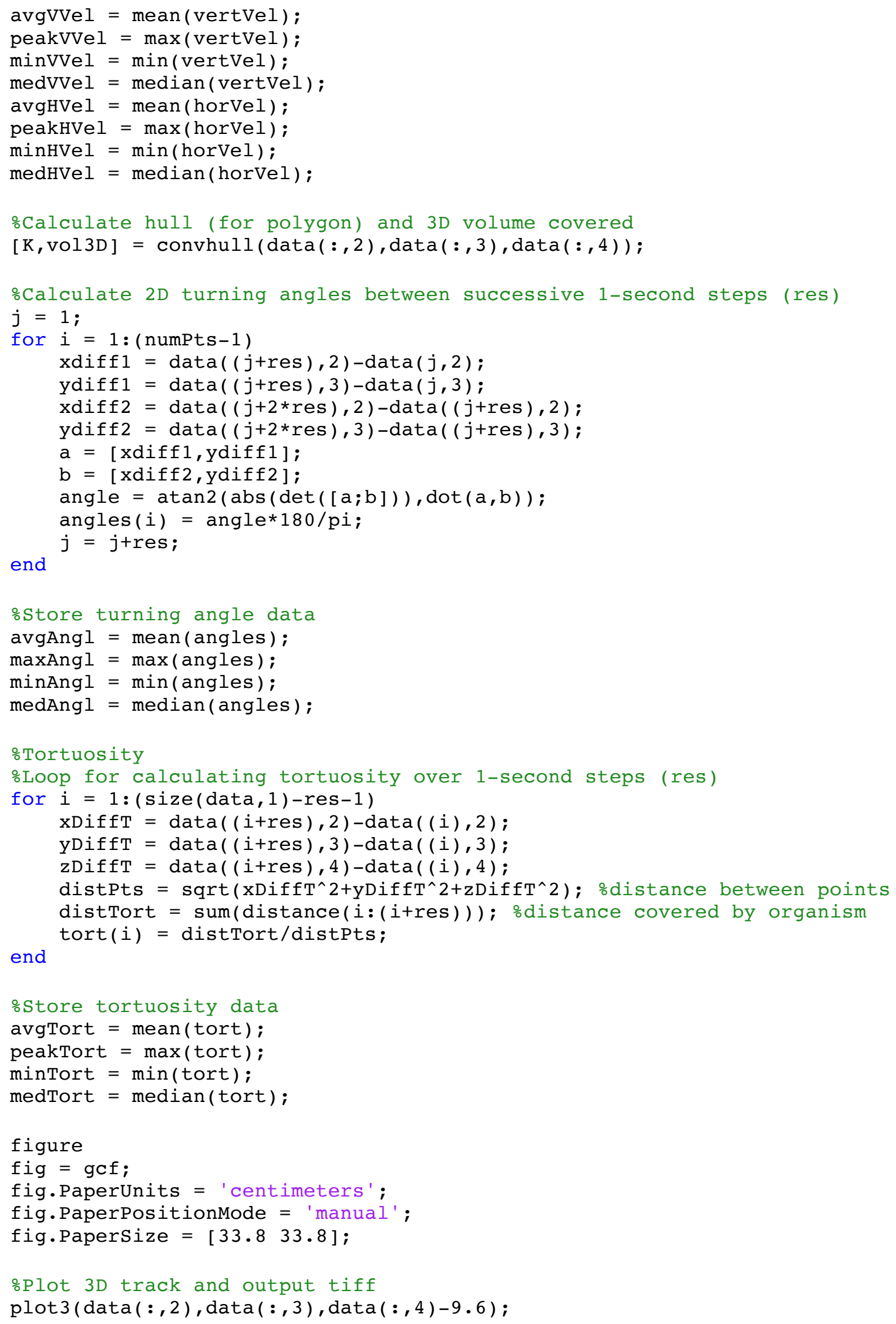




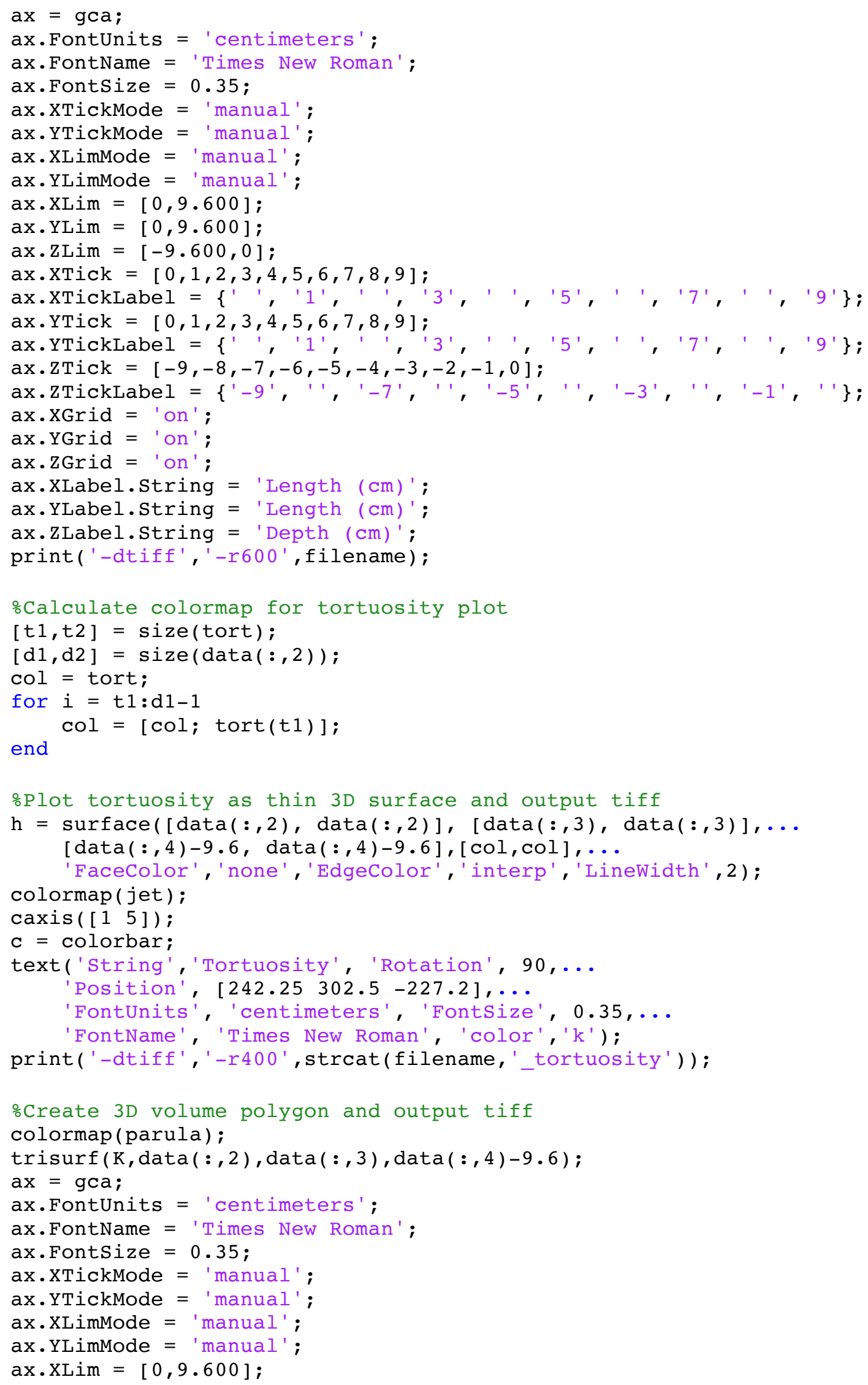




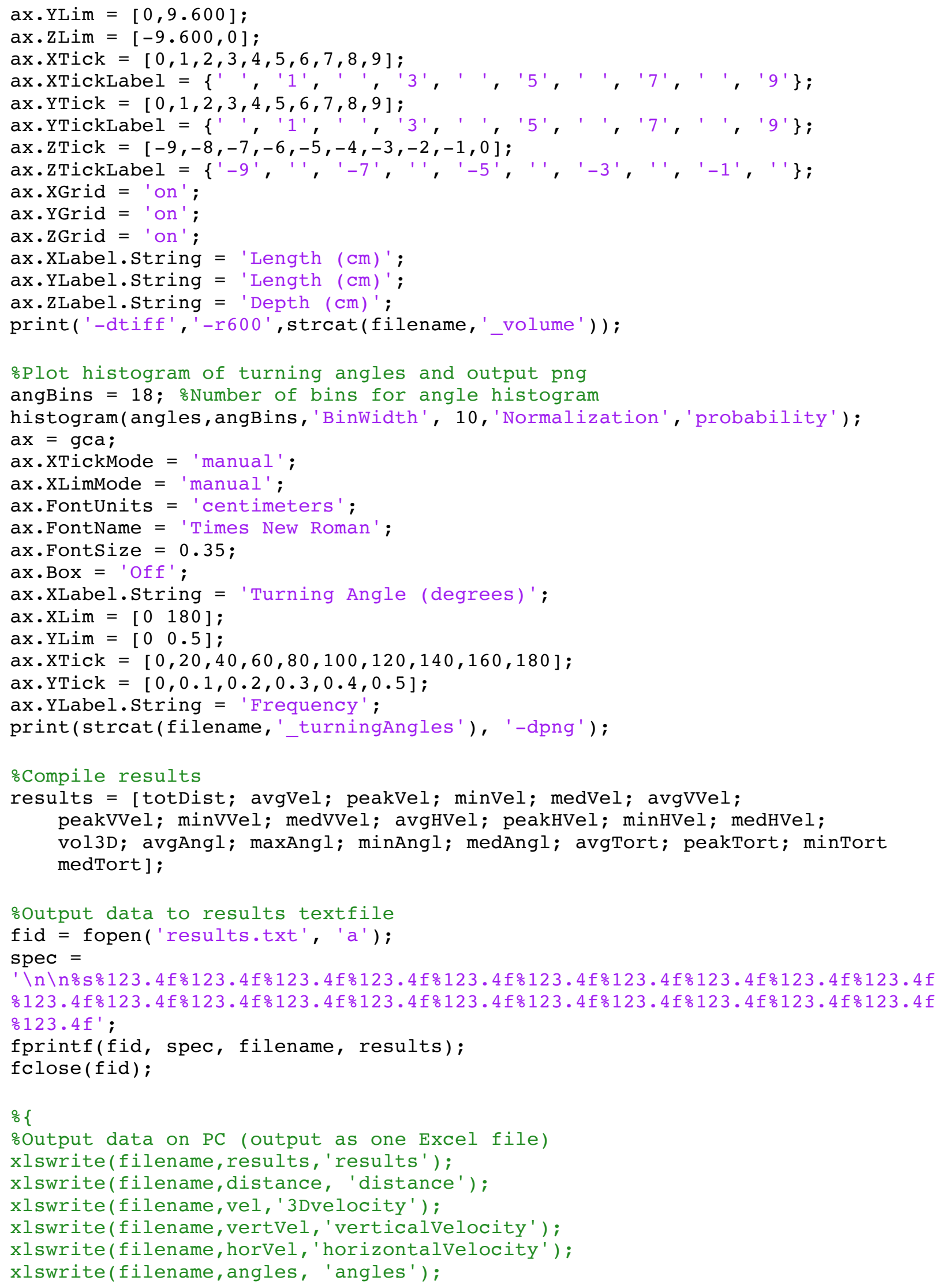


xlswrite(filename, tort, 'tortuosity');

\%

oOutput data on Mac (output as individual CSVs)

xlswrite (strcat (filename, '_results'), results);

xlswrite (strcat (filename, '_distance' ), distance);

xlswrite (strcat (filename, '_3Dvelocity'), vel);

xlswrite (strcat (filename, '_verticalvelocity'), vertvel);

xlswrite (strcat (filename, '_horizontalvelocity'), horvel);

xlswrite (strcat (filename, '_angles '), angles);

xlswrite (strcat (filename, '_tortuosity'), tort);

end 


\section{Appendix C}

\section{Chapter 4 Supplementary Materials}

In preparation for submission to Global Change Biology. 

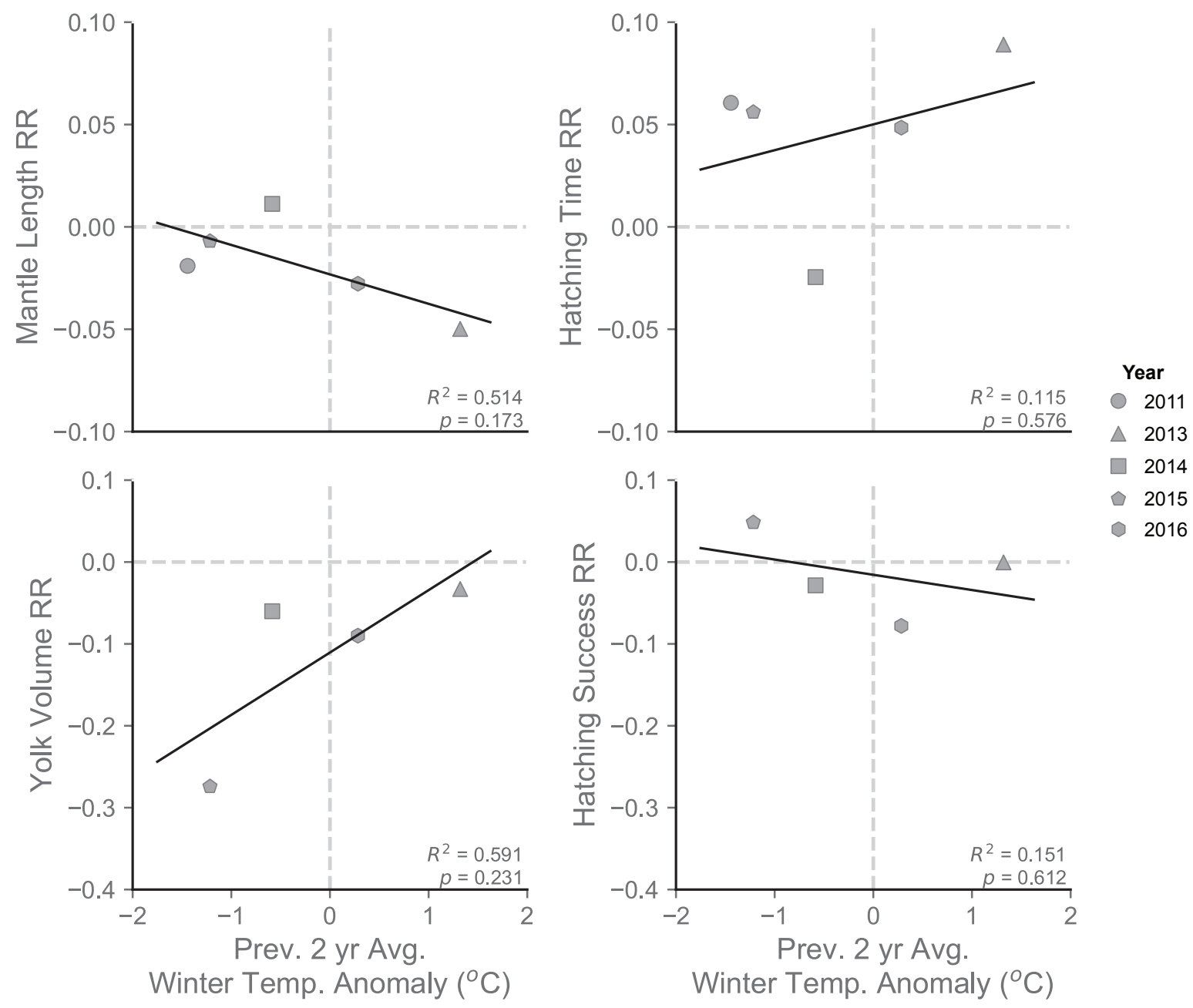

Figure S1. Log-transformed response ratios, compiled by year, for each metric plotted against the winter temperature anomaly (average anomaly for Dec-Feb) averaged for the previous two winters in Vineyard Sound. Symbols represent mean response ratios for each year; error is not shown for visual clarity. Shape corresponds to year. Lines represent linear regressions, with $\mathrm{R}^{2}$ and $p$ value reported at the bottom right of each graph 

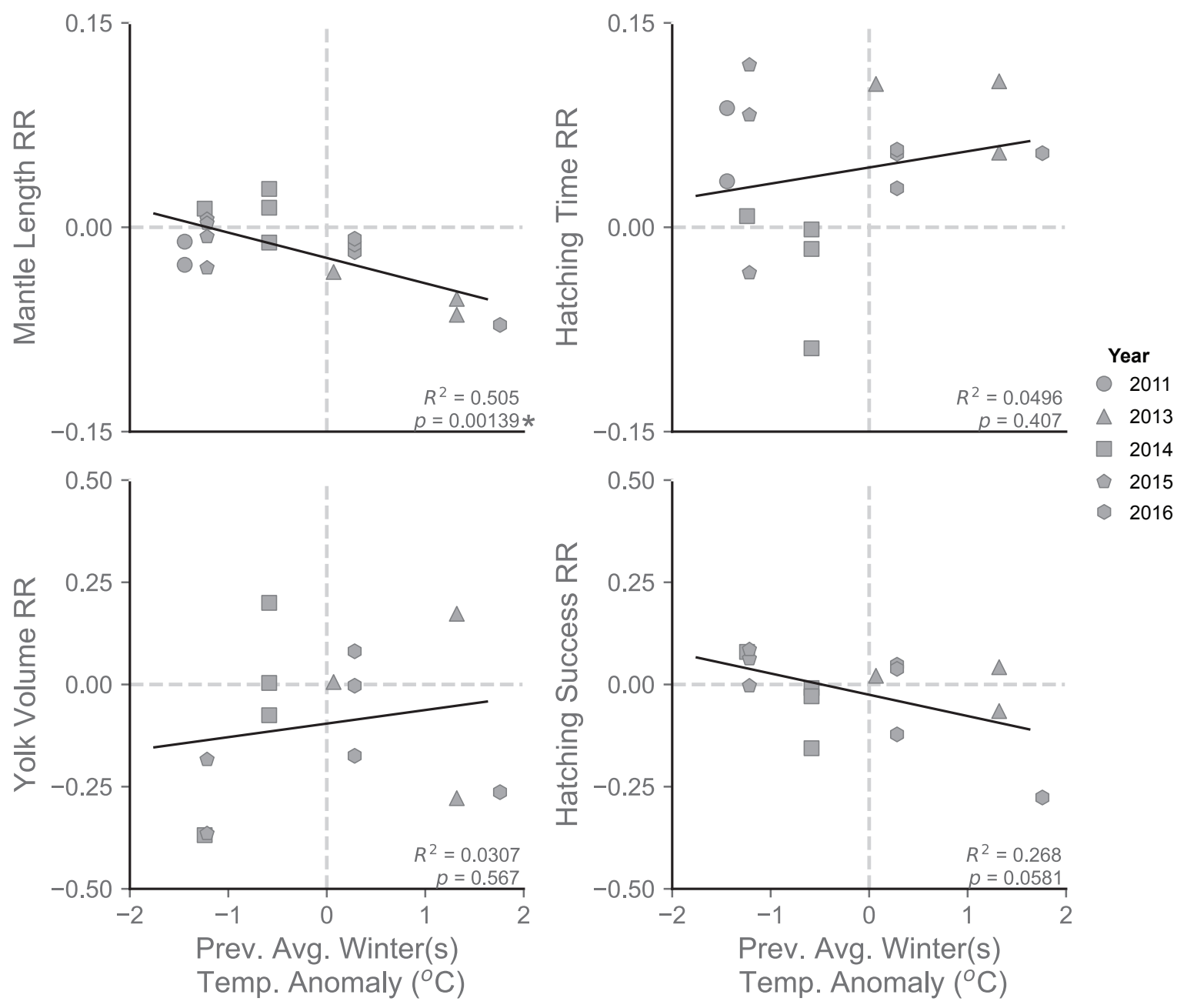

Figure S2. Log-transformed response ratios for each trial and metric plotted against the winter temperature anomaly (average anomaly for Dec-Feb) in Vineyard Sound experienced by adults (Apr - Jul: 2 year previous winter average; Aug - Oct: 1 year previous winter average) for the hypothesized population succession outlined in Mesnil (1977). Symbols represent mean response ratios for each year. Shape corresponds to year. Lines represent linear regressions, with $\mathbf{R}^{2}$ and $p$ value reported at the bottom right of each graph. Significant $p$ values $(\alpha=0.05)$ are marked with an asterisk 


\section{Appendix D}

\section{Chapter 5 Supplementary Materials}

This chapter is a pre-print of a submitted manuscript, authored by Zakroff, CJ and Mooney, TA, to a special issue of Frontiers in Physiology: Invertebrate Physiology: Cephalopod Research Across Scales - Molecules to Ecosystems. 
Table S1. Hatching success distributions and G-tests. Counts of staged, failed embryos and paralarvae, presented by trial and treatment. Staged embryos were categorized using Arnold et al. (1974) as Early (embryonic stages 1 - 16), Middle (17 - 26), or Late $(27-30)$. G-tests are reported with significant $p$-values $(p<0.05)$ in bold. Exponents of the $p$-value are listed to compare degrees of significance among comparisons with $p$-values less than 0.001

\begin{tabular}{|c|c|c|c|c|c|c|c|c|c|c|c|}
\hline $\begin{array}{l}\text { Temperature } \\
\left({ }^{\circ} \mathrm{C}\right)\end{array}$ & $\mathrm{pCO}_{2}(\mathrm{ppm})$ & Early & Middle & Late & Hatched & $\begin{array}{l}\text { Percent } \\
\text { Hatched }\end{array}$ & Source & $G$ & $d f$ & $\mathrm{p}$ & $\exp$ \\
\hline \multicolumn{12}{|l|}{ Jun 19} \\
\hline \multirow{4}{*}{20} & 400 & 1 & 1 & 1 & 273 & 98.9 & $400 \times 2200$ & 22.01 & 3 & $<0.001$ & -05 \\
\hline & 2200 & 5 & 0 & 20 & 310 & 92.7 & $400 \times 3500$ & 90.45 & 3 & $<0.001$ & -19 \\
\hline & 3500 & 2 & 2 & 48 & 132 & 73.2 & $2200 \times 3500$ & 45.16 & 3 & $<0.001$ & -10 \\
\hline & & & & & & & All $20^{\circ} \mathrm{C}$ & 100.6 & 6 & $<0.001$ & -19 \\
\hline \multirow{4}{*}{27} & 400 & 5 & 4 & 4 & 314 & 96.1 & $400 \times 2200$ & 158.4 & 3 & $<0.001$ & -34 \\
\hline & 2200 & 9 & 105 & 18 & 190 & 59.2 & $400 \times 3500$ & 27.45 & 3 & $<0.001$ & -06 \\
\hline & 3500 & 7 & 6 & 22 & 183 & 84.1 & $2200 \times 3500$ & 87.82 & 3 & $<0.001$ & -19 \\
\hline & & & & & & & All $27^{\circ} \mathrm{C}$ & 205.0 & 6 & $<0.001$ & -41 \\
\hline \multirow{3}{*}{$20 \times 27$} & & & & & & & $400 \times 400$ & 5.313 & 3 & 0.150 & -01 \\
\hline & & & & & & & $2200 \times 2200$ & 175.7 & 3 & $<0.001$ & -38 \\
\hline & & & & & & & $3500 \times 3500$ & 20.34 & 3 & $<0.001$ & -04 \\
\hline \multicolumn{12}{|l|}{ Jul 28} \\
\hline \multirow{4}{*}{20} & 400 & 3 & 1 & 19 & 473 & 95.4 & $400 \times 2200$ & 20.21 & 3 & $<0.001$ & -04 \\
\hline & 2200 & 3 & 0 & 2 & 562 & 99.1 & $400 \times 3500$ & 10.77 & 3 & 0.013 & -02 \\
\hline & 3500 & 0 & 0 & 28 & 323 & 92.1 & $2200 \times 3500$ & 45.10 & 3 & $<0.001$ & -10 \\
\hline & & & & & & & All $20^{\circ} \mathrm{C}$ & 48.08 & 6 & $<0.001$ & -08 \\
\hline \multirow{4}{*}{27} & 400 & 135 & 45 & 59 & 144 & 34.0 & $400 \times 2200$ & 317.5 & 3 & $<0.001$ & -86 \\
\hline & 2200 & 1 & 3 & 18 & 330 & 93.2 & $400 \times 3500$ & 134.4 & 3 & $<0.001$ & -68 \\
\hline & 3500 & 10 & 19 & 93 & 165 & 54.7 & $2200 \times 3500$ & 126.4 & 3 & $<0.001$ & -29 \\
\hline & & & & & & & All $27^{\circ} \mathrm{C}$ & 414.1 & 6 & $<0.001$ & -27 \\
\hline \multirow{3}{*}{$20 \times 27$} & & & & & & & $400 \times 400$ & 408.4 & 3 & $<0.001$ & -88 \\
\hline & & & & & & & $2200 \times 2200$ & 30.20 & 3 & $<0.001$ & -06 \\
\hline & & & & & & & $3500 \times 3500$ & 122.7 & 3 & $<0.001$ & -26 \\
\hline \multicolumn{12}{|l|}{ Sep 14} \\
\hline \multirow{4}{*}{20} & 400 & 71 & 0 & 0 & 385 & 85.1 & $400 \times 2200$ & 257.6 & 3 & $<0.001$ & -55 \\
\hline & 2200 & 21 & 7 & 119 & 181 & 57.8 & $400 \times 3500$ & 179.0 & 3 & $<0.001$ & -39 \\
\hline & 3500 & 10 & 0 & 85 & 275 & 74.2 & $2200 \times 3500$ & 36.37 & 3 & $<0.001$ & -08 \\
\hline & & & & & & & All $20^{\circ} \mathrm{C}$ & 299.9 & 6 & $<0.001$ & -62 \\
\hline \multirow{4}{*}{27} & 400 & 15 & 2 & 4 & 346 & 94.0 & $400 \times 2200$ & 3.524 & 3 & 0.318 & -01 \\
\hline & 2200 & 21 & 0 & 4 & 375 & 93.8 & $400 \times 3500$ & 172.3 & 3 & $<0.001$ & -37 \\
\hline & 3500 & 165 & 4 & 13 & 231 & 56.8 & $2200 \times 3500$ & 171.6 & 3 & $<0.001$ & -37 \\
\hline & & & & & & & All $27^{\circ} \mathrm{C}$ & 252.0 & 6 & $<0.001$ & -51 \\
\hline \multirow{3}{*}{$20 \times 27$} & & & & & & & $400 \times 400$ & 40.37 & 3 & $<0.001$ & -09 \\
\hline & & & & & & & $2200 \times 2200$ & 206.9 & 3 & $<0.001$ & -44 \\
\hline & & & & & & & $3500 \times 3500$ & 232.1 & 3 & $<0.001$ & -50 \\
\hline
\end{tabular}


Table S2. Malformation distributions and G-tests. Counts of normal and malformed paralarvae, presented by trial and treatment. Malformed paralarvae were categorized as Premature (external yolk remaining), Eye Bulge (inflated eye membrane), or Malformed Head (malformations/growths on head and mantle). G-tests are reported with significant $p$-values $(p<0.05)$ in bold. Exponents of the $p$-value are listed to compare degrees of significance among comparisons with $p$-values less than 0.001 .

\begin{tabular}{|c|c|c|c|c|c|c|c|c|c|c|}
\hline $\begin{array}{l}\text { Temperature } \\
\left({ }^{\circ} \mathrm{C}\right)\end{array}$ & $\mathrm{pCO}_{2}(\mathrm{ppm})$ & Normal & Premature & $\begin{array}{l}\text { Eye } \\
\text { Bulge }\end{array}$ & $\begin{array}{l}\text { Malformed } \\
\text { Head }\end{array}$ & Source & $G$ & df & $\mathrm{p}$ & $\exp$ \\
\hline \multicolumn{11}{|l|}{ Jun 19} \\
\hline \multirow{4}{*}{20} & 400 & 168 & 6 & 2 & 2 & $400 \times 2200$ & 12.51 & 3 & 0.006 & -03 \\
\hline & 2200 & 190 & 20 & 12 & 1 & $400 \times 3500$ & 139.7 & 3 & $<0.001$ & -30 \\
\hline & 3500 & 27 & 43 & 21 & 5 & $2200 \times 3500$ & 99.8 & 3 & $<0.001$ & -21 \\
\hline & & & & & & All $20^{\circ} \mathrm{C}$ & 155.7 & 6 & $<0.001$ & -31 \\
\hline \multirow{4}{*}{27} & 400 & 179 & 4 & 3 & 18 & $400 \times 2200$ & 25.15 & 3 & $<0.001$ & -05 \\
\hline & 2200 & 61 & 7 & 8 & 21 & $400 \times 3500$ & 101.7 & 3 & $<0.001$ & -22 \\
\hline & 3500 & 77 & 34 & 39 & 16 & $2200 \times 3500$ & 25.85 & 3 & $<0.001$ & -05 \\
\hline & & & & & & All $27^{\circ} \mathrm{C}$ & 112.4 & 6 & $<0.001$ & -22 \\
\hline \multirow{3}{*}{$20 \times 27$} & & & & & & $400 \times 400$ & 13.90 & 3 & 0.003 & -03 \\
\hline & & & & & & $2200 \times 2200$ & 48.29 & 3 & $<0.001$ & -10 \\
\hline & & & & & & $3500 \times 3500$ & 18.73 & 3 & $<0.001$ & -04 \\
\hline \multicolumn{11}{|l|}{ Jul 28} \\
\hline \multirow{4}{*}{20} & 400 & 202 & 13 & 8 & 3 & $400 \times 2200$ & 22.77 & 3 & $<0.001$ & -05 \\
\hline & 2200 & 260 & 28 & 48 & 9 & $400 \times 3500$ & 137.2 & 3 & $<0.001$ & -29 \\
\hline & 3500 & 46 & 63 & 21 & 14 & $2200 \times 3500$ & 105.8 & 3 & $<0.001$ & -23 \\
\hline & & & & & & All $20^{\circ} \mathrm{C}$ & 166.8 & 6 & $<0.001$ & -33 \\
\hline \multirow{4}{*}{27} & 400 & 121 & 25 & 6 & 1 & $400 \times 2200$ & 15.49 & 3 & 0.001 & -03 \\
\hline & 2200 & 148 & 20 & 31 & 1 & $400 \times 3500$ & 61.25 & 3 & $<0.001$ & -13 \\
\hline & 3500 & 45 & 54 & 17 & 12 & $2200 \times 3500$ & 73.33 & 3 & $<0.001$ & -16 \\
\hline & & & & & & All $27^{\circ} \mathrm{C}$ & 99.58 & 6 & $<0.001$ & -19 \\
\hline \multirow{3}{*}{$20 \times 27$} & & & & & & $400 \times 400$ & 11.57 & 3 & 0.009 & -03 \\
\hline & & & & & & $2200 \times 2200$ & 4.485 & 3 & 0.214 & -01 \\
\hline & & & & & & $3500 \times 3500$ & 0.338 & 3 & 0.953 & -01 \\
\hline \multicolumn{11}{|l|}{ Sep 14} \\
\hline \multirow{4}{*}{20} & 400 & 234 & 8 & 10 & 3 & $400 \times 2200$ & 30.94 & 3 & $<0.001$ & -07 \\
\hline & 2200 & 125 & 14 & 31 & 1 & $400 \times 3500$ & 241.1 & 3 & $<0.001$ & -52 \\
\hline & 3500 & 48 & 98 & 44 & 7 & $2200 \times 3500$ & 111.9 & 3 & $<0.001$ & -24 \\
\hline & & & & & & All $20^{\circ} \mathrm{C}$ & 266.6 & 6 & $<0.001$ & -54 \\
\hline \multirow{4}{*}{27} & 400 & 210 & 7 & 0 & 0 & $400 \times 2200$ & 44.30 & 3 & $<0.001$ & -09 \\
\hline & 2200 & 161 & 18 & 20 & 4 & $400 \times 3500$ & 216.0 & 3 & $<0.001$ & -46 \\
\hline & 3500 & 55 & 64 & 41 & 11 & $2200 \times 3500$ & 89.74 & 3 & $<0.001$ & -19 \\
\hline & & & & & & All $27^{\circ} \mathrm{C}$ & 227.3 & 6 & $<0.001$ & -46 \\
\hline \multirow{3}{*}{$20 \times 27$} & & & & & & $400 \times 400$ & 16.32 & 3 & $<0.001$ & -04 \\
\hline & & & & & & $2200 \times 2200$ & 6.622 & 3 & 0.085 & -02 \\
\hline & & & & & & $3500 \times 3500$ & 6.829 & 3 & 0.078 & -02 \\
\hline
\end{tabular}




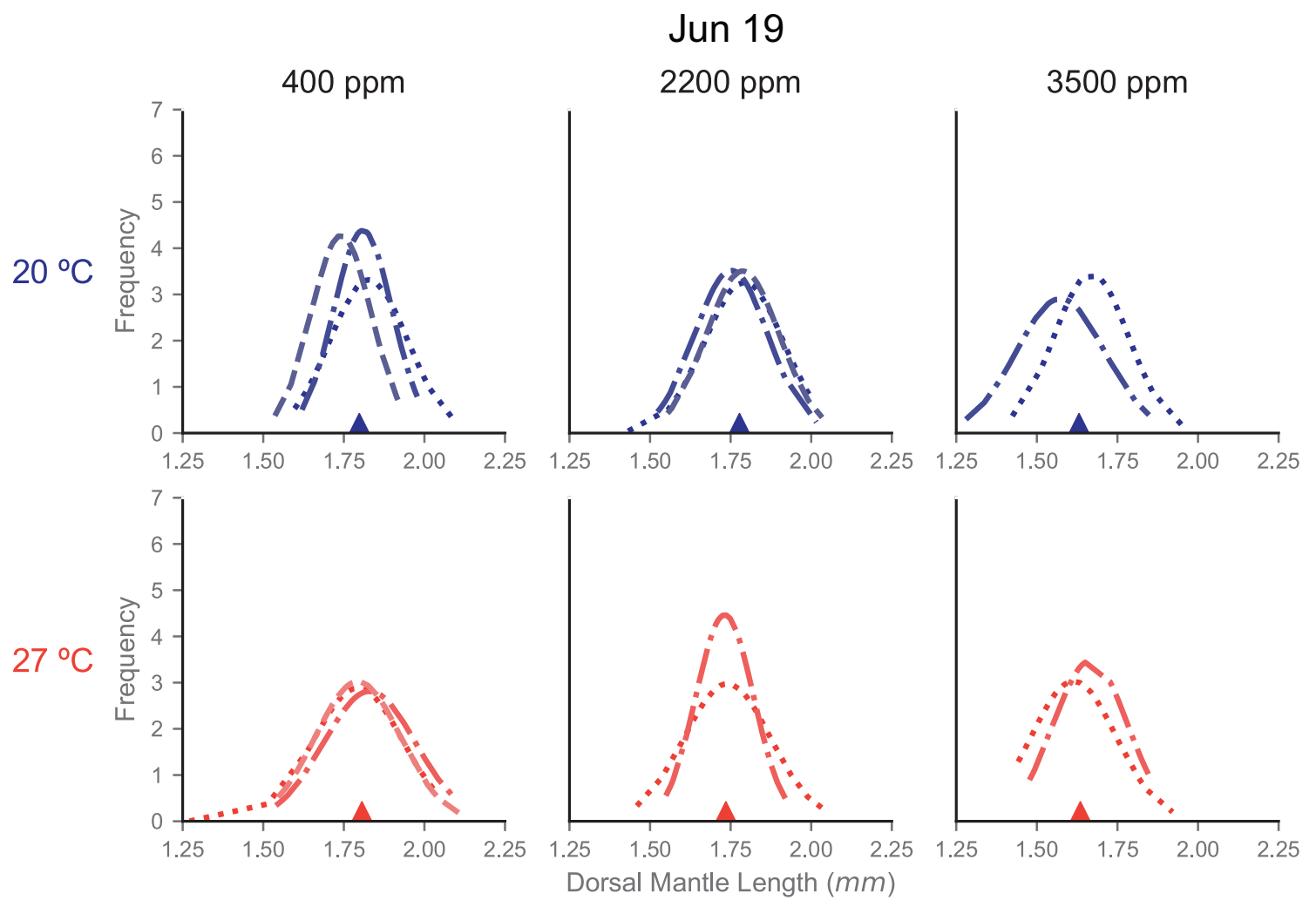

Figure S1. Distributions of mantle length from individual egg capsules of the June 19 trial. Fitted normal distributions of dorsal mantle length frequency histograms from individual egg capsules in each treatment of the June 19 trial. Histograms are not shown for clarity of curves, but DML data were segmented in $0.05 \mathrm{~mm}$ bins. Each plot is a treatment combination, with column determining acidification (titles) and row determining temperature treatment (also differentiated by color: top $/$ blue $=20^{\circ} \mathrm{C}$, bottom $/ \mathrm{red}=27^{\circ} \mathrm{C}$ ). Each line represents the curve from the sampling of an individual egg capsule for dorsal mantle length (x-axis). Lines are shaded and patterned to help differentiate individual egg capsules within a plot, but this carries no relationship or meaning across the plots. The filled triangle on the $\mathrm{x}$-axis marks the mean value for the compiled sample of that treatment 


\section{Jul 28}
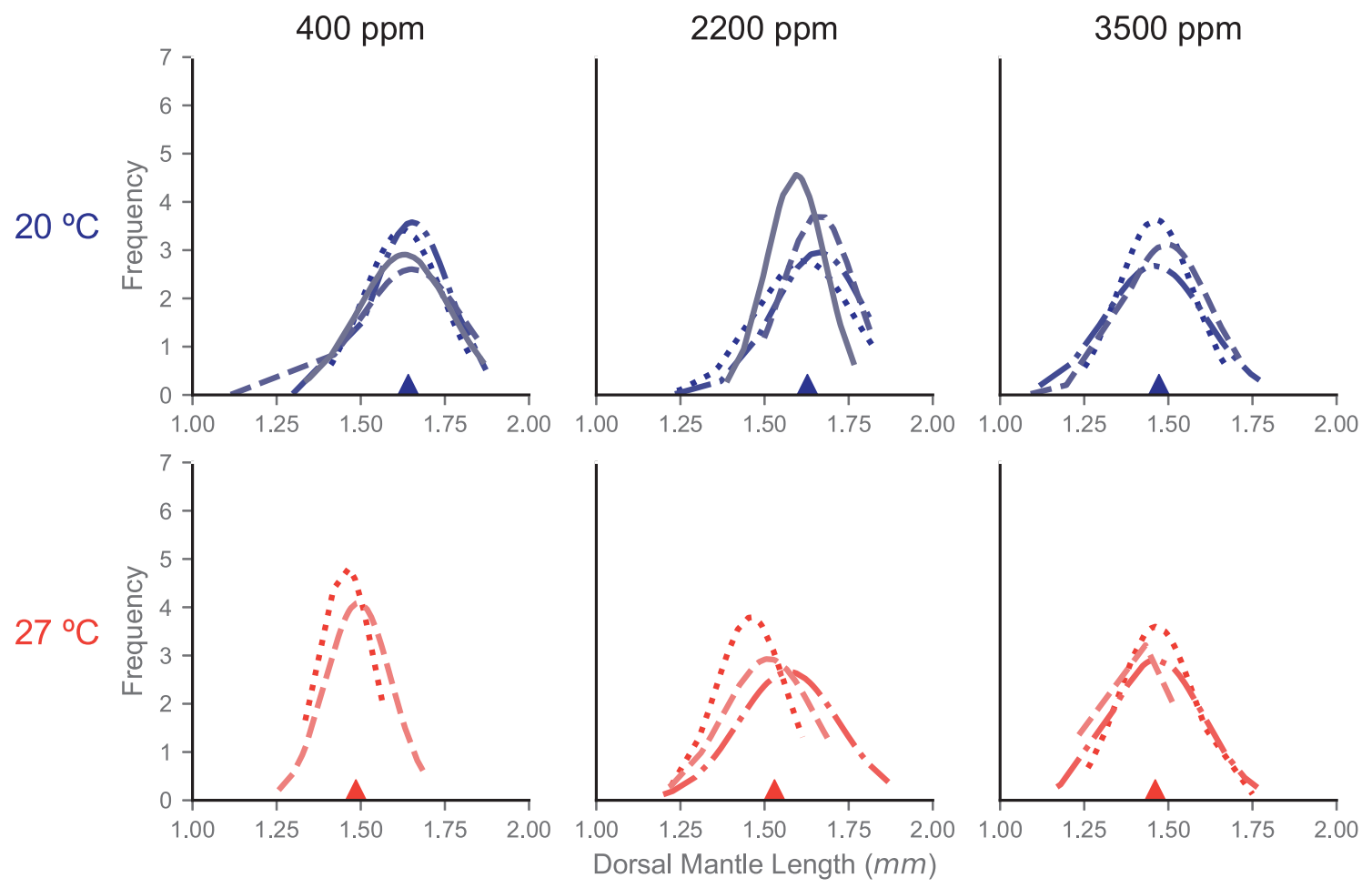

Figure S2. Distributions of mantle length from individual egg capsules of the July 28 trial. Fitted normal distributions of dorsal mantle length frequency histograms from individual egg capsules in each treatment of the July 28 trial. Histograms are not shown for clarity of curves, but DML data were segmented in $0.05 \mathrm{~mm}$ bins. Each plot is a treatment combination, with column determining acidification (titles) and row determining temperature treatment (also differentiated by color: top/blue $=20^{\circ} \mathrm{C}$, bottom $/ \mathrm{red}=27^{\circ} \mathrm{C}$ ). Each line represents the curve from the sampling of an individual egg capsule for dorsal mantle length (x-axis). Lines are shaded and patterned to help differentiate individual egg capsules within a plot, but this carries no relationship or meaning across the plots. The filled triangle on the $\mathrm{x}$-axis marks the mean value for the compiled sample of that treatment 


\section{BIBLIOGRAPHY}

Aiken B, Man JPH (1978) Positive phototaxis of the brine shrimp Artemia salina to monochromatic light.

Albertin CB, Bonnaud L, Brown CT, Crookes-Goodson WJ, da Fonseca RR, Di Cristo C, Dilkes BP, Edsinger-Gonzales E, Freeman RM, Hanlon RT, Koenig KM, Lindgren AR, Martindale MQ, Minx P, Moroz LL, Nödl M-T, Nyholm S V., Ogura A, Pungor JR, Rosenthal JJC, Schwarz EM, Shigeno S, Strugnell JM, Wollesen T, Zhang G, Ragsdale CW (2012) Cephalopod genomics: A plan of strategies and organization. Stand Genomic Sci 7:175188. doi: 10.4056/sigs. 3136559

Albertin CB, Simakov O, Mitros T, Wang ZY, Pungor JR, Edsinger-Gonzales E, Brenner S, Ragsdale CW, Rokhsar DS (2015) The octopus genome and the evolution of cephalopod neural and morphological novelties. Nature 524:220-224. doi: 10.1038/nature14668

Alon S, Garrett SC, Levanon EY, Olson S, Graveley BR, Rosenthal JJC, Eisenberg E (2015) The majority of transcripts in the squid nervous system are extensively recoded by A-to-I RNA editing. Elife. doi: 10.7554/eLife.05198

Anthony KRN, Kline DI, Diaz-Pulido G, Dove S, Hoegh-Guldberg O (2008) Ocean acidification causes bleaching and productivity loss in coral reef builders. Proc Natl Acad Sci 105:17442-17446. doi: 10.1073/pnas.0804478105

Appelhans Y, Thomsen J, Pansch C, Melzner F, Wahl M (2012) Sour times: seawater acidification effects on growth, feeding behaviour and acid-base status of Asterias rubens and Carcinus maenas. Mar Ecol Prog Ser 459:85-98. doi: 10.3354/meps09697

Arkhipkin AI (2003) Towards identification of the ecological lifestyle in nektonic squid using statolith morphometry. J Molluscan Stud 69:171-178. doi: 10.1093/mollus/69.3.171

Arkhipkin AI, Bizikov VA (1997) Statolith shape and microstructure in studies of systematics, age and growth in planktonic paralarvae of gonatid squids (Cephalopoda, Oegopsida) from the western Bering Sea. J Plankton Res 19:1993-2030. doi: 10.1093/plankt/19.12.1993

Arkhipkin AI, Bizikov VA (2000) Role of the statolith in functioning of the acceleration receptor system in squids and sepioids. J Zool 250:31-55. doi: 10.1111/j.1469-7998.2000.tb00575.x

Arnold JM, Summers WC, Gilbert DL, Manalis RS, Daw NW, Lasek RJ (1974) A guide to laboratory use of the squid Loligo pealei. Marine Biological Laboratory, Woods Hole, MA

Atkinson D (1994) Temperature and organism size - a biological law for ectotherms? Adv Ecol Res 25:1-58.

Auster PJ, Shackell NL (2000) Marine protected areas for the temperate and boreal northwest atlantic: The potential for sustainable fisheries and conservation of biodiversity. Northeast Nat 7:419-434.

Barón PJ (2003) The paralarvae of two South American sympatric squid: Loligo gahi and Loligo sanpaulensis. J Plankton Res 25:1347-1358. doi: 10.1093/plankt/fbg093

Bartol IK, Krueger PS, Thompson JT, Stewart WJ (2008) Swimming dynamics and propulsive efficiency of squids throughout ontogeny. Integr Comp Biol 48:720-733. doi: 10.1093/icb/icn043 
Bartol IK, Krueger PS, Stewart WJ, Thompson JT (2009a) Hydrodynamics of pulsed jetting in juvenile and adult brief squid Lolliguncula brevis: evidence of multiple jet "modes" and their implications for propulsive efficiency. J Exp Biol 212:1889-1903. doi: 10.1242/jeb.027771

Bartol IK, Krueger PS, Stewart WJ, Thompson JT (2009b) Pulsed jet dynamics of squid hatchlings at intermediate Reynolds numbers. J Exp Biol 212:1506-1518. doi: $10.1242 /$ jeb.033241

Baumann H, Wallace RB, Tagliaferri T, Gobler CJ (2015) Large Natural pH, CO2 and O2 Fluctuations in a Temperate Tidal Salt Marsh on Diel, Seasonal, and Interannual Time Scales. Estuaries and Coasts 38:220-231. doi: 10.1007/s12237-014-9800-y

Baumann H, Cross EL, Murray CS (2018) Robust quantification of fish early life CO2 sensitivities via serial experimentation.

Beck MW, Heck KL, Able KW, Childers DL, Eggleston DB, Gillanders BM, Halpern B, Hays CG, Hoshino K, Minello TJ, Orth RJ, Sheridan PF, Weinstein MP (2001) The identification, conservation, and management of estuarine and marine nurseries for fish and invertebrates. Bioscience 51:633-641. doi: 10.1641/00063568(2001)051[0633:TICAMO]2.0.CO;2

Benoit-Bird KJ, Gilly WF (2012) Coordinated nocturnal behavior of foraging jumbo squid Dosidicus gigas. Mar Ecol Prog Ser 455:211-228. doi: 10.3354/meps09664

Birk MA, McLean EL, Seibel BA (2018) Ocean acidification does not limit squid metabolism via blood oxygen supply. J Exp Biol jeb.187443. doi: 10.1242/jeb.187443

Boisclair D (1992) An Evaluation of the Stereocinematographic Method to Estimate Fish Swimming Speed. Can J Fish Aquat Sci 49:523-531.

Bonduriansky R, Crean AJ (2018) What are parental condition-transfer effects and how can they be detected? Methods Ecol Evol 9:450-456. doi: 10.1111/2041-210X.12848

Bonhomme V, Picq S, Gaucherel C, Claude J (2013) Momocs: outline analysis using R. J Stat Softw 56:1-24. doi: 10.18637/jss.v056.i13

Boyle PR, Pierce GJ, Hastie LC (1995) Flexible reproductive strategies in the squid Loligo forbesi. Mar Biol 121:501-508.

Breitburg DL, Salisbury J, Bernhard JM, Cai W-J, Dupont S, Doney SC, Kroeker KJ, Levin LA, Long WC, Milke LM, Miller SH, Phelan B, Passow U, Seibel BA, Todgham AE, Tarrant AM (2015) And on top of all that... Coping with ocean acidification in the midst of many stressors. Oceanography 28:48-61. doi: http://dx.doi.org/10.5670/oceanog.2015.31

Brodziak J, Hendrickson L (1999) An analysis of environmental effects on survey catches of squids Loligo pealei and Illex illecebrosus in the northwest Atlantic. Fish Bull 97:9-24.

Browman HI (2016) Applying organized scepticism to ocean acidification research. ICES J Mar Sci 73:529-536. doi: 10.1126/science.158.3803.950

Budick SA, O’Malley DM (2000) Locomotor repertoire of the larval zebrafish: swimming, turning and prey capture. J Exp Biol 203:2565-2579.

Buresch KC, Gerlach G, Hanlon RT (2006) Multiple genetic stocks of longfin squid Loligo pealeii in the NW Atlantic: stocks segregate inshore in summer, but aggregate offshore in 
winter. Mar Ecol Prog Ser 310:263-270. doi: 10.3354/meps310263

Buresch KC, Maxwell MR, Cox MR, Hanlon RT (2009) Temporal dynamics of mating and paternity in the squid Loligo pealeii. Mar Ecol Prog Ser 387:197-203. doi: 10.3354/meps08052

Buresch KM, Hanlon RT, Maxwell MR, Ring S (2001) Microsatellite DNA markers indicate a high frequency of multiple paternity within individual field-collected egg capsules of the squid Loligo pealeii. Mar Ecol Prog Ser 210:161-165.

Burgess SC, Marshall DJ (2011) Temperature-induced maternal effects and environmental predictability. 2329-2336. doi: 10.1242/jeb.054718

Byrne M (2011) Impact of ocean warming and ocean acidification on marine invertebrate life history stages: Vulnerabilities and potential for persistence in a changing ocean. Ocean Mar Biol Annu Rev 49:1-42. doi: doi:10.1016/j.marenvres.2011.10.00

Cachat J, Stewart A, Utterback E, Hart P, Gaikwad S, Wong K, Kyzar E, Wu N, Kalueff A V. (2011a) Three-dimensional neurophenotyping of adult zebrafish behavior. PLoS One. doi: 10.1371/journal.pone.0017597

Cachat JM, Canavello PR, Elkhayat SI, Bartels BK, Hart PC, Elegante MF, Esther C, Laffoon AL, Haymore WAM, Tien DH, Tien AK, Mohnot S, Kalueff A V (2011b) Chapter 16: Deconstructing Adult Zebrafish Behavior with Swim Trace Visualizations. In: Kalueff A V., Cachat JM (eds) Zebrafish Neurobehavioral Protocols. pp 191-201

Caldeira K, Wickett ME (2003) Oceanography: anthropogenic carbon and ocean pH. Nature 425:365. doi: $10.1038 / 425365 a$

Campus P (1999) Response of Squids to Different Colours and Intensities of Artificial Light. 22:19-24.

Carré M, Bentaleb I, Bruguier O, Ordinola E, Barrett NT, Fontugne M (2006) Calcification rate influence on trace element concentrations in aragonitic bivalve shells: Evidences and mechanisms. Geochim Cosmochim Acta 70:4906-4920. doi: 10.1016/j.gca.2006.07.019

Cattano C, Claudet J, Domenici P, Milazzo M (2018) Living in a high $\mathrm{CO}_{2}$ world: a global metaanalysis shows multiple trait-mediated fish responses to ocean acidification. Ecol Monogr 88:320-335. doi: $10.1002 / \mathrm{ecm} .1297$

Chan KYK, Grünbaum D, Arnberg M, Thorndyke M, Dupont ST (2013) Ocean acidification induces budding in larval sea urchins. Mar Biol 160:2129-2135. doi: 10.1007/s00227-0122103-6

Chan KYK, García E, Dupont S (2015) Acidification reduced growth rate but not swimming speed of larval sea urchins. Sci Rep 5:9764. doi: 10.1038/srep09764

Checkley DM, Dickson AG, Takahashi M, Radich JA, Eisenkolb N, Asch R (2009) Elevated $\mathrm{CO} 2$ enhances otolith growth in young fish. Science 324:1683. doi:

10.1126/science. 1169806

Cheung WWL, Lam VWY, Pauly D (2008) Dynamic bioclimate envelope model to predict climate-induced changes in distribution of marine fishes and invertebrates. Fish Cent Res Rep 16 16:5-50.

Cheung WWL, Lam VWY, Sarmiento JL, Kearney K, Watson R, Pauly D (2009) Projecting 
global marine biodiversity impacts under climate change scenarios. Fish Fish 10:235-251. doi: 10.1111/j.1467-2979.2008.00315.x

Chung W, Marshall NJ, Watson S, Munday PL, Nilsson GE (2014) Ocean acidification slows retinal function in a damselfish through interference with GABAA receptors. J Exp Biol 217:323-6. doi: 10.1242/jeb.092478

Cinti A, Barón PJ, Rivas AL (2004) The effects of environmental factors on the embryonic survival of the Patagonian squid Loligo gahi. J Exp Mar Bio Ecol 313:225-240. doi: 10.1016/j.jembe.2004.05.017

Clayton TD, Byrne RH (1993) Spectrophotometric seawater pH measurements: total hydrogen results. Deep Res 40:2115-2129.

Cohen AL, Holcomb M (2009) Why corals care about ocean acidification: Uncovering the mechanism. Oceanography 22:118-127. doi: 10.5670/oceanog.2009.102

Coll M, Navarro J, Olson RJ, Christensen V (2013) Assessing the trophic position and ecological role of squids in marine ecosystems by means of food-web models. Deep Res Part II Top Stud Oceanogr 95:21-36. doi: 10.1016/j.dsr2.2012.08.020

Collins MA, Burnell GM, Rodhouse PG (1995) Reproductive strategies of male and female Loligo forbesi (Cephalopoda: Loliginidae). J Mar Biol Assoc UK 75:621-634.

Colmers WF, Hixon RF, Hanlon RT, Forsythe JW, Ackerson M V., Wiederhold ML, Hulet WH (1984) Spinner cephalopods: defects of statocyst suprastructures in an invertebrate analogue of the vestibular apparatus. Cell Tissue Res. doi: 10.1007/BF00217217

Comeau S, Carpenter RC, Lantz C a., Edmunds PJ (2015) Ocean acidification accelerates dissolution of experimental coral reef communities. Biogeosciences 12:365-372. doi: $10.5194 /$ bg-12-365-2015

Connolly TP, Lentz SJ (2014) Interannual variability of wintertime temperature on the inner continental shelf of the Middle Atlantic Bight. J Geophys Res Ocean 119:6269-6285. doi: 10.1002/2014JC010153

Coughlin DJ, Strickler JR, Sanderson B (1992) Swimming and Search Behavior in Clownfish, Amphiprion-Perideraion, Larvae. Anim Behav 44:427-440.

Cowen RK, Sponaugle S (2009) Larval dispersal and marine population connectivity. Ann Rev Mar Sci 1:443-466. doi: 10.1146/annurev.marine.010908.163757

Crain CM, Kroeker K, Halpern BS (2008) Interactive and cumulative effects of multiple human stressors in marine systems. Ecol Lett 11:1304-1315. doi: 10.1111/j.14610248.2008.01253.x

Crean AJ, Marshall DJ (2009) Coping with environmental uncertainty: Dynamic bet hedging as a maternal effect. Philos Trans R Soc B Biol Sci 364:1087-1096. doi: 10.1098/rstb.2008.0237

DeCarlo TM, Cohen AL, Barkley HC, Cobban Q, Young C, Shamberger KE, Brainard RE, Golbuu Y (2015a) Coral macrobioerosion is accelerated by ocean acidification and nutrients. Geology 43:7-10. doi: 10.1130/G36147.1

DeCarlo TM, Gaetani GA, Holcomb M, Cohen AL (2015b) Experimental determination of factors controlling $\mathrm{U} / \mathrm{Ca}$ of aragonite precipitated from seawater: Implications for interpreting coral skeleton. Geochim Cosmochim Acta 162:151-165. doi: 


\subsection{6/j.gca.2015.04.016}

Dickson AG (1990) Standard potential of the reaction: $\mathrm{AgCl}(\mathrm{s})+(1 / 2) \mathrm{H}_{2}(\mathrm{~g})=\mathrm{Ag}(\mathrm{s})+\mathrm{HCl}(\mathrm{aq})$, and and the standard acidity constant of the ion $\mathrm{HSO}_{4}{ }^{-}$in synthetic sea water from 273.15 to 318.15 K. J Chem Thermodyn 22:113-127. doi: 10.1016/0021-9614(90)90074-Z

Dickson AG, Sabine CL, Christian JR (2007) Guide to best practices for ocean $\mathrm{CO}_{2}$ measurements. PICES Spec Publ 3:p191. doi: 10.1159/000331784

Dissard D, Nehrke G, Reichart GJ (2009) Impact of seawater pCO2 changes on calcification and on $\mathrm{mG} / \mathrm{cA}$ and $\mathrm{sR} / \mathrm{cA}$ in benthic foraminifera calcite ( Ammonia tepida ): results from culturing experiments. 3771-3802.

Dixson DL, Munday PL, Jones GP (2010) Ocean acidification disrupts the innate ability of fish to detect predator olfactory cues. Ecol Lett 13:68-75. doi: 10.1111/j.14610248.2009.01400.x

Dixson DL, Jennings AR, Atema J, Munday PL (2015) Odor tracking in sharks is reduced under future ocean acidification conditions. Glob Chang Biol 21:1454-1462. doi:

$10.1111 / \mathrm{gcb} .12678$

Donelson JM, McCormick MI, Munday PL (2008) Parental condition affects early life-history of a coral reef fish. J Exp Mar Bio Ecol 360:109-116. doi: 10.1016/j.jembe.2008.04.007

Donelson JM, Munday PL, Mccormick MI (2009) Parental effects on offspring life histories : when are they important? 262-265. doi: 10.1098/rsbl.2008.0642

Doney SC, Fabry VJ, Feely RA, Kleypas JA (2009) Ocean acidification: the other $\mathrm{CO}_{2}$ problem. Ann Rev Mar Sci 1:169-192. doi: 10.1146/annurev.marine.010908.163834

Dorey N, Melzner F, Martin S, Oberhänsli F, Teyssié JL, Bustamante P, Gattuso JP, LacoueLabarthe T (2013) Ocean acidification and temperature rise: Effects on calcification during early development of the cuttlefish Sepia officinalis. Mar Biol 160:2007-2022. doi: $10.1007 / \mathrm{s} 00227-012-2059-6$

Doubleday ZA, Prowse TAA, Arkhipkin A, Pierce GJ, Semmens J, Steer M, Leporati SC, Lourenço S, Quetglas A, Sauer W, Gillanders BM (2016) Global proliferation of cephalopods. Curr Biol 26:R406-R407. doi: 10.1016/j.cub.2016.04.002

Drenkard EJ, Cohen AL, McCorkle DC, de Putron SJ, Starczak VR, Zicht AE (2013) Calcification by juvenile corals under heterotrophy and elevated CO2. Coral Reefs 32:727735. doi: 10.1007/s00338-013-1021-5

Dupont S, Ortega-Martínez O, Thorndyke M (2010) Impact of near-future ocean acidification on echinoderms. Ecotoxicology 19:449-62. doi: 10.1007/s10646-010-0463-6

Emery AM, Wilson IJ, Craig S, Boyle PR, Noble LR (2001) Assignment of paternity groups without access to parental genotypes: multiple mating and developmental plasticity in squid. Mol Ecol 10:1265-78. doi: 10.1046/j.1365-294X.2001.01258.x

Fabioux C, Huvet A, Lelong C, Robert R, Pouvreau S, Daniel JY, Minguant C, Le Pennec M (2004a) Oyster vasa-like gene as a marker of the germline cell development in Crassostrea gigas. Biochem Biophys Res Commun 320:592-598. doi: 10.1016/j.bbrc.2004.06.009

Fabioux C, Pouvreau S, Le Roux F, Huvet A (2004b) The oyster vasa-like gene: A specific marker of the germline in Crassostrea gigas. Biochem Biophys Res Commun 315:897-904. 
doi: $10.1016 / j . b b r c .2004 .01 .145$

Fabry VJ, Seibel BA, Feely RA, Orr JC (2008) Impacts of ocean acidification on marine fauna and ecosystem processes. ICES J Mar Sci 65:414. doi: 10.1093/icesjms/fsn048

Feely RA, Alin SR, Newton J, Sabine CL, Warner M, Devol A, Krembs C, Maloy C (2010) The combined effects of ocean acidification, mixing, and respiration on $\mathrm{pH}$ and carbonate saturation in an urbanized estuary. Estuar Coast Shelf Sci 88:442-449. doi:

10.1016/j.ecss.2010.05.004

Fernández-Reiriz M, Range P, Álvarez-Salgado X, Espinosa J, Labarta U (2012) Tolerance of juvenile Mytilus galloprovincialis to experimental seawater acidification. Mar Ecol Prog Ser 454:65-74. doi: 10.3354/meps09660

Ferrari MCO, McCormick MI, Munday PL, Meekan MG, Dixson DL, Lonnstedt Ö, Chivers DP (2011) Putting prey and predator into the $\mathrm{CO} 2$ equation - qualitative and quantitative effects of ocean acidification on predator-prey interactions. Ecol Lett 14:1143-1148. doi: 10.1111/j.1461-0248.2011.01683.x

Findlay HS, Kendall MA, Spicer JI, Widdicombe S (2010) Relative influences of ocean acidification and temperature on intertidal barnacle post-larvae at the northern edge of their geographic distribution. Estuar Coast Shelf Sci 86:675-682. doi: 10.1016/j.ecss.2009.11.036

Flickinger AL, Bruins RJF, Winner RW, Skillings JH (1982) Filtration and phototactic behavior as indices of chronic copper stress in Daphnia magna Straus. Arch Environ Contam Toxicol 11:457-463. doi: 10.1007/BF01056072

Franco-Santos RM, Perales-Raya C, Almansa E, De Troch M, Garrido D (2016) Beak microstructure analysis as a tool to identify potential rearing stress for Octopus vulgaris paralarvae. Aquac Res 47:3001-3015. doi: 10.1111/are.12753

Freitas PS, Clarke LJ, Kennedy H, Richardson CA, Abrantes F (2006) Environmental and biological controls on elemental $(\mathrm{Mg} / \mathrm{Ca}, \mathrm{Sr} / \mathrm{Ca}$ and $\mathrm{Mn} / \mathrm{Ca})$ ratios in shells of the king scallop Pecten maximus. Geochim Cosmochim Acta 70:5119-5133. doi: 10.1016/j.gca.2006.07.029

Fuchs HL, Mullineaux LS, Solow AR (2004) Sinking behavior of gastropod larvae (Ilyanassa obsoleta) in turbulence. Limnol Oceanogr 49:1937-1948. doi: 10.4319/lo.2004.49.6.1937

Gaetani GA, Cohen AL (2006) Element partitioning during precipitation of aragonite from seawater: A framework for understanding paleoproxies. Geochim Cosmochim Acta 70:4617-4634. doi: 10.1016/j.gca.2006.07.008

Gallager SM, Mann R, Sasaki GC (1986) Lipid as an index of growth and viability in three species of bivalve larvae. Aquaculture 56:81-103. doi: 10.1016/0044-8486(86)90020-7

García-Fernández P, García-Souto D, Almansa E, Morán P, Gestal C (2017) Epigenetic DNA methylation mediating Octopus vulgaris early development: Effect of essential fatty acids enriched diet. Front Physiol. doi: 10.3389/fphys.2017.00292

Gazeau F, Quiblier C, Jansen JM, Gattuso J-P, Middelburg JJ, Heip CHR (2007) Impact of elevated CO2 on shellfish calcification. Geophys Res Lett. doi: 10.1029/2006GL028554

Gazeau F, Gattuso JP, Dawber C, Pronker AE, Peene F, Peene J, Heip CHR, Middelburg JJ 
(2010) Effect of ocean acidification on the early life stages of the blue mussel Mytilus edulis. Biogeosciences 7:2051-2060. doi: 10.5194/bg-7-2051-2010

Gazeau F, Parker LM, Comeau S, Gattuso J-PP, O'Connor WA, Martin S, Pörtner H-O, Ross PM (2013) Impacts of ocean acidification on marine shelled molluscs. Mar Biol 160:22072245. doi: 10.1007/s00227-013-2219-3

Gledhill DK, White MM, Salisbury J, Thomas H, Misna I, Liebman M, Mook B, Grear J, Candelmo AC, Chambers RC, Gobler CJ, Hunt CW, King AL, Price NN, Signorini SR, Stancioff E, Stymiest C, Wahle RA, Waller JD, Rebuck ND, Wang ZA, Capson TL, Morrison JR, Cooley SR, Doney SC (2015) Ocean and coastal acidification off New England and Nova Scotia. Oceanography 28:182-197. doi:

http://dx.doi.org/10.5670/oceanog.2015.41

González AF, Otero J, Guerra A, Prego R, Rocha FJ, Dale AW (2005) Distribution of common octopus and common squid paralarvae in a wind-driven upwelling area (Ria of Vigo, northwestern Spain). J Plankton Res 27:271-277. doi: 10.1093/plankt/fbi001

Gray CL (1992) Long-finned squid (Loligo pealei) species profile. In: Current Report: The Narragansett Bay Project NBP-92-106. Rhode Island Department of Environmental Management, Division of Fish \& Wildlife, Marine Fisheries Section, pp 1-54

Guerra Á, Allcock L, Pereira J (2010) Cephalopod life history, ecology and fisheries: An introduction. Fish Res 106:117-124. doi: 10.1016/j.fishres.2010.09.002

Guillaume AS, Monro K, Marshall DJ (2016) Transgenerational plasticity and environmental stress: do paternal effects act as a conduit or a buffer? Funct Ecol 30:1175-1184. doi: $10.1111 / 1365-2435.12604$

Guppy M, Withers P (1999) Metabolic depression in animals: physiological perspectives and biochemical generalizations. Biol Rev Camb Philos Soc 74:1-40. doi: $10.1017 / \mathrm{s} 0006323198005258$

Gutowska MA, Melzner F (2009) Abiotic conditions in cephalopod (Sepia officinalis) eggs: Embryonic development at low pH and high pCO2. Mar Biol 156:515-519. doi: 10.1007/s00227-008-1096-7

Gutowska MA, Pörtner HO, Melzner F (2008) Growth and calcification in the cephalopod Sepia officinalis under elevated seawater pCO2. Mar Ecol Prog Ser 373:303-309. doi: 10.3354/meps07782

Gutowska MA, Melzner F, Langenbuch M, Bock C, Claireaux G, Pörtner HO (2010a) Acid-base regulatory ability of the cephalopod (Sepia officinalis) in response to environmental hypercapnia. J Comp Physiol B 180:323-335. doi: 10.1007/s00360-009-0412-y

Gutowska MA, Melzner F, Pörtner HO, Meier S (2010b) Cuttlebone calcification increases during exposure to elevated seawater pCO2 in the cephalopod Sepia officinalis. Mar Biol 157:1653-1663. doi: 10.1007/s00227-010-1438-0

Haigh R, Ianson D, Holt CA, Neate HE, Edwards AM (2015) Effects of ocean acidification on temperate coastal marine ecosystems and fisheries in the northeast Pacific. PLoS One 10:e0117533. doi: 10.1371/journal.pone.0117533

Hamdoun A, Epel D (2007) Embryo stability and vulnerability in an always changing world. Proc Natl Acad Sci 104:1745-1750. doi: 10.1073/pnas.0610108104 
Hanlon R, Bidwell J, Tait R (1989) Strontium is required for statolith development and thus normal swimming behaviour of hatchling cephalopods. J Exp Biol 141:187-195.

Hanlon RT, Messenger JB (1998) Cephalopod Behaviour. Cambridge University Press, Cambridge, UK

Hanlon RT, Hixon RF, Hulet WH (1983) Survival, growth, and behavior of the loliginid squids Lologi plei, Loligo pealei, and Lolliguncula brevis (Mollusca: Cephalopoda) in closed sea water systems. Biol Bull 637-685. doi: 10.2307/1541470

Harvey BP, Gwynn-Jones D, Moore PJ (2013) Meta-analysis reveals complex marine biological responses to the interactive effects of ocean acidification and warming. Ecol Evol 3:10161030. doi: 10.1002/ece3.516

Hastie LC, Pierce GJ, Wang J, Bruno I, Moreno A (2009) Cephalopods in the North-Eastern Atlantic: Species, Biogeography, Ecology, Exploitation and Conservation. Oceanogr Mar Biol An Annu Rev 47:111-190. doi: 10.1201/9781420094220.ch3

Hatfield EMC, Cadrin SX (2002) Geographic and temporal patterns in size and maturity of the longfin inshore squid (Loligo pealeii) off the northeastern United States. Fish Bull 100:200213.

Haury L, Weihs D (1976) Energetically efficient swimming behavior of negatively buoyant zooplankton. Limnol Oceanogr 21:797-803. doi: 10.4319/1o.1976.21.6.0797

Hendriks IE, Duarte CM, Olsen YS, Steckbauer A, Ramajo L, Moore TS, Trotter J a., McCulloch M (2015) Biological mechanisms supporting adaptation to ocean acidification in coastal ecosystems. Estuar Coast Shelf Sci 152:A1-A8. doi: 10.1016/j.ecss.2014.07.019

Herke SW, Foltz DW (2002) Phylogeography of two squid (Loligo pealei and L. plei) in the Gulf of Mexico and northwestern Atlantic Ocean. Mar Biol 140:103-115. doi: $10.1007 / \mathrm{s} 002270100680$

Hidu H, Haskin HH (1978) Swimming speeds of oyster larvae Crassostrea virginica in different salinities and temperatures. Estuaries 1:252-255. doi: 10.1007/BF02762480

Hobday AJ, Pecl GT (2014) Identification of global marine hotspots: Sentinels for change and vanguards for adaptation action. Rev Fish Biol Fish 24:415-425. doi: 10.1007/s11160-0139326-6

Hobday AJ, Cochrane K, Downey-Breedt N, Howard J, Aswani S, Byfield V, Duggan G, Duna E, Dutra LXC, Frusher SD, Fulton EA, Gammage L, Gasalla MA, Griffiths C, Guissamulo A, Haward M, Jarre A, Jennings SM, Jordan T, Joyner J, Ramani NK, Shanmugasundaram SLP, Malherbe W, Cisneros KO, Paytan A, Pecl GT, Plagányi ÉE, Popova EE, Razafindrainibe H, Roberts M, Rohit P, Sainulabdeen SS, Sauer W, Valappil ST, Zacharia PU, van Putten EI (2016) Planning adaptation to climate change in fast-warming marine regions with seafood-dependent coastal communities. Rev Fish Biol Fish 26:249-264. doi: 10.1007/s11160-016-9419-0

Hofmann GE, Todgham AE (2010) Living in the now: Physiological mechanisms to tolerate a rapidly changing environment. Annu Rev Physiol 72:127-145. doi: 10.1146/annurevphysiol-021909-135900

Holcomb M, Cohen AL, Gabitov RI, Hutter JL (2009) Compositional and morphological features of aragonite precipitated experimentally from seawater and biogenically by corals. 
Geochim Cosmochim Acta 73:4166-4179. doi: 10.1016/j.gca.2009.04.015

Holcomb M, Mccorkle DC, Cohen AL (2010) Journal of Experimental Marine Biology and Ecology Long-term effects of nutrient and $\mathrm{CO} 2$ enrichment on the temperate coral Astrangia. J Exp Mar Bio Ecol 386:27-33. doi: 10.1016/j.jembe.2010.02.007

Holcomb M, Venn AA, Tambutté E, Tambutté S, Allemand D, Trotter J, McCulloch M (2014) Coral calcifying fluid $\mathrm{pH}$ dictates response to ocean acidification. Sci Rep 4:1-4. doi: 10.1038/srep05207

Hong X, Martin PJ, Wang S, Rowley C (2009) High SST variability south of Martha's Vineyard: Observation and modeling study. J Mar Syst 78:59-76. doi: 10.1016/j.jmarsys.2009.03.001

Honisch B, Ridgwell A, Schmidt DN, Thomas E, Gibbs SJ, Sluijs A, Zeebe R, Kump L, Martindale RC, Greene SE, Kiessling W, Ries J, Zachos JC, Royer DL, Barker S, Marchitto TM, Moyer R, Pelejero C, Ziveri P, Foster GL, Williams B (2012) The Geological Record of Ocean Acidification. Science (80- ) 335:1058-1063. doi: 10.1126/science.1208277

Hoving HJT, Gilly WF, Markaida U, Benoit-Bird KJ, Brown ZW, Daniel P, Field JC, Parassenti L, Liu B, Campos B (2013) Extreme plasticity in life-history strategy allows a migratory predator (jumbo squid) to cope with a changing climate. Glob Chang Biol 19:2089-2103. doi: $10.1111 / \mathrm{gcb} .12198$

Hu M, Tseng Y-C (2017) Acid--Base Regulation and Ammonia Excretion in Cephalopods: An Ontogenetic Overview. In: Weihrauch D, O’Donnell M (eds) Acid-Base Balance and Nitrogen Excretion in Invertebrates: Mechanisms and Strategies in Various Invertebrate Groups with Considerations of Challenges Caused by Ocean Acidification. Springer International Publishing, Cham, pp 275-298

Hu MY, Sucre E, Charmantier-Daures M, Charmantier G, Lucassen M, Himmerkus N, Melzner F (2010) Localization of ion-regulatory epithelia in embryos and hatchlings of two cephalopods. Cell Tissue Res 339:571-583.

Hu MY, Tseng Y-C, Stumpp M, Gutowska MA, Kiko R, Lucassen M, Melzner F (2011a) Elevated seawater $\mathrm{pCO}_{2}$ differentially affects branchial acid-base transporters over the course of development in the cephalopod Sepia officinalis. Am J Physiol Regul Integr Comp Physiol 300:R1100-R1114. doi: 10.1152/ajpregu.00653.2010

Hu MY, Tseng Y-C, Lin L-Y, Chen P-Y, Charmantier-Daures M, Hwang P-P, Melzner F (2011b) New insights into ion regulation of cephalopod molluscs: a role of epidermal ionocytes in acid-base regulation during embryogenesis. AJP Regul Integr Comp Physiol 301:R1700-R1709. doi: 10.1152/ajpregu.00107.2011

Hu MY, Lee J-R, Lin L-Y, Shih T-H, Stumpp M, Lee M-F, Hwang P-P, Tseng Y-C (2013) Development in a naturally acidified environment: $\mathrm{Na}^{+} / \mathrm{H}^{+}$-exchanger 3-based proton secretion leads to $\mathrm{CO}_{2}$ tolerance in cephalopod embryos. Front Zool 10:51. doi: 10.1186/1742-9994-10-51

Hu MY, Guh Y-J, Stumpp M, Lee J-R, Chen R-D, Sung P-H, Chen Y-C, Hwang P-P, Tseng Y-C (2014) Branchial $\mathrm{NH}_{4}{ }^{+}$-dependent acid-base transport mechanisms and energy metabolism of squid (Sepioteuthis lessoniana) affected by seawater acidification. Front Zool 11:55. doi: 10.1186/s12983-014-0055-z

Hunsicker ME, Essington TE (2006) Size-structured patterns of piscivory of the longfin inshore 
squid (Loligo pealeii) in the mid-Atlantic continental shelf ecosystem. Can J Fish Aquat Sci 63:754-765. doi: 10.1139/f05-258

Hunsicker ME, Essington TE (2008) Evaluating the potential for trophodynamic control of fish by the longfin inshore squid (Loligo pealeii) in the Northwest Atlantic Ocean. Can J Fish Aquat Sci 65:2524-2535. doi: 10.1139/F08-154

Ikeda Y, Wada Y, Arai N, Sakamoto W (1999) Note on size variation of body and statoliths in the oval squid Sepioteuthis lessoniana hatchlings. J Mar Biol Assoc UK 79:757-759. doi: $10.1017 / \mathrm{S} 0025315498000939$

Jacobson LD (2005) Longfin inshore squid, Loligo pealeii, life history and habitat characteristics. In: NOAA Technical Memorandum NMFS-NE-193. U.S. Department of Commerce, National Oceanic and Atmospheric Administration, National Marine Fisheries Service, Northeast Fisheries Science Center, Woods Hole, MA, pp 1-42

Jensen N, Allen RM, Marshall DJ (2014) Adaptive maternal and paternal effects: Gamete plasticity in response to parental stress. Funct Ecol 28:724-733. doi: 10.1111/13652435.12195

Jin Y, Lin F, Chen X, Liu B, Li J (2019) Microstructure comparison of hard tissues (statoliths, beaks, and eye lenses) of Uroteuthis chinensis in the South China Sea. Bull Mar Sci 95:1326.

Juárez OE, Galindo-Sánchez CE, Díaz F, Re D, Sánchez-García AM, Camaal-Monsreal C, Rosas C (2015) Is temperature conditioning Octopus maya fitness? J Exp Mar Bio Ecol 467:7176. doi: $10.1016 /$ j.jembe.2015.02.020

Kaplan MB, Mooney TA, McCorkle DC, Cohen AL (2013) Adverse effects of ocean acidification on early development of squid (Doryteuthis pealeii). PLoS One 8:e63714. doi: 10.1371/journal.pone.0063714

Keyl F, Argüelles J, Tafur R (2011) Interannual variability in size structure, age, and growth of jumbo squid (Dosidicus gigas) assessed by modal progression analysis. ICES J Mar Sci 68:507-518. doi: 10.1093/icesjms/fsq167

Kingston ACN, Wardill TJ, Hanlon RT, Cronin TW (2015) An Unexpected Diversity of Photoreceptor Classes in the Longfin Squid, Doryteuthis pealeii. PLoS One 10:e0135381. doi: 10.1371/journal.pone.0135381

Klimley AP, Brown ST (1983) Stereophotography for the field biologist: measurement of lengths and three-dimensional positions of free-swimming sharks. Mar Biol 74:175-185. doi: 10.1007/BF00413921

Kroeker KJ, Kordas RL, Crim RN, Singh GG (2010) Meta-analysis reveals negative yet variable effects of ocean acidification on marine organisms. Ecol Lett 13:1419-1434. doi: 10.1111/j.1461-0248.2010.01518.x

Kroeker KJ, Kordas RL, Crim R, Hendriks IE, Ramajo L, Singh GS, Duarte CM, Gattuso JP (2013) Impacts of ocean acidification on marine organisms: Quantifying sensitivities and interaction with warming. Glob Chang Biol 19:1884-1896. doi: 10.1111/gcb.12179

Kroeker KJ, Sanford E, Jellison BM, Gaylord B (2014) Predicting the effects of ocean acidification on predator-prey interactions: a conceptual framework based on coastal molluscs. Biol Bull 226:211-222. 
Kroeker KJ, Kordas RL, Harley CDG (2017) Embracing interactions in ocean acidification research: confronting multiple stressor scenarios and context dependence. Biol Lett 13:20160802. doi: 10.1098/rsbl.2016.0802

Lacoue-Labarthe T, Warnau M, Oberhänsli F, Teyssié JL, Koueta N, Bustamante P (2008) Differential bioaccumulation behaviour of $\mathrm{Ag}$ and $\mathrm{Cd}$ during the early development of the cuttlefish Sepia officinalis. Aquat Toxicol 86:437-446. doi: 10.1016/j.aquatox.2007.12.005

Lacoue-Labarthe T, Réveillac E, Oberhänsli F, Teyssié JL, Jeffree R, Gattuso JP (2011) Effects of ocean acidification on trace element accumulation in the early-life stages of squid Loligo vulgaris. Aquat Toxicol 105:166-176. doi: 10.1016/j.aquatox.2011.05.021

Lacoue-Labarthe T, Martin S, Oberhänsli F, Teyssié JL, Jeffree R, Gattuso JP, Bustamante P (2012) Temperature and $\mathrm{pCO}_{2}$ effect on the bioaccumulation of radionuclides and trace elements in the eggs of the common cuttlefish, Sepia officinalis. J Exp Mar Bio Ecol 413:45-49. doi: 10.1016/j.jembe.2011.11.025

Langenbuch M, Pörtner HO (2002) Changes in metabolic rate and N excretion in the marine invertebrate Sipunculus nudus under conditions of environmental hypercapnia: identifying effective acid-base variables. J Exp Biol 205:1153-1160.

Langsrud Ø (2003) ANOVA for unbalanced data: Use Type II instead of Type III sums of squares. Stat Comput 13:163-167. doi: 10.1023/A:1023260610025

Laptikhovsky V V., Rogov MA, Nikolaeva S V., Arkhipkin AI (2013) Environmental impact on ectocochleate cephalopod reproductive strategies and the evolutionary significance of cephalopod egg size. Bull Geosci 88:83-93. doi: 10.3140/bull.geosci.1351

Laptikhovsky V, Nikolaeva S, Rogov M (2018) Cephalopod embryonic shells as a tool to reconstruct reproductive strategies in extinct taxa. Biol Rev 93:270-283. doi: $10.1111 /$ brv. 12341

Lee PN, McFall-Ngai MJ, Callaerts P, de Couet HG (2009) The hawaiian bobtail squid (Euprymna scolopes): A model to study the molecular basis of eukaryote-prokaryote mutualism and the development and evolution of morphological novelties in cephalopods. Cold Spring Harb Protoc 4:1-10. doi: 10.1101/pdb.emo135

Leporati SC, Pecl GT, Semmens JM (2007) Cephalopod hatchling growth: The effects of initial size and seasonal temperatures. Mar Biol 151:1375-1383. doi: 10.1007/s00227-006-0575-y

Levin L, Honisch B, Frieder C (2015) Geochemical proxies for estimating faunal exposure to ocean acidification. Oceanography 28:62-73. doi: 10.5670/oceanog.2015.32

Lipinski MR (1993) The deposition of statoliths: a working hypothesis. In: Okutani T, O’Dor RK, Kubodera T (eds) Recent Advances in Cephalopods Fisheries Biology. Tokai University Press, Tokyo, Japan, pp 241-262

Lischka S, Büdenbender J, Boxhammer T, Riebesell U (2011) Impact of ocean acidification and elevated temperatures on early juveniles of the polar shelled pteropod Limacina helicina: Mortality, shell degradation, and shell growth. Biogeosciences 8:919-932. doi: 10.5194/bg8-919-2011

Liscovitch-Brauer N, Alon S, Porath HT, Elstein B, Unger R, Ziv T, Admon A, Levanon EY, Rosenthal JJC, Eisenberg E (2017) Trade-off between transcriptome plasticity and genome evolution in cephalopods. Cell 169:191-202.e11. doi: 10.1016/j.cell.2017.03.025 
Long MH, Mooney TA, Zakroff C (2016) Extreme low oxygen and decreased pH conditions naturally occur within developing squid egg capsules. Mar Ecol Prog Ser 550:111-119. doi: $10.3354 /$ meps 11737

Lunden JJ, McNicholl CG, Sears CR, Morrison CL, Cordes E (2014) Acute survivorship of the deep-sea coral Lophelia pertusa from the Gulf of Mexico under acidification, warming, and deoxygenation. Front Mar Sci 1:74. doi: 10.3389/fmars.2014.00078

Maas AE, Wishner KF, Seibel BA (2012) The metabolic response of pteropods to acidification reflects natural CO2-exposure in oxygen minimum zones. Biogeosciences 9:747-757. doi: DOI 10.5194/bg-9-747-2012

Macy III WK (1982) Feeding patterns of the long-finned squid, Loligo pealei, in New England waters. Biol Bull 162:28-38. doi: 10.2307/1540967

Macy III WK, Brodziak JKT (2001) Seasonal maturity and size at age of Loligo pealeii in waters of southern New England. ICES J Mar Sci 58:852-864. doi: 10.1006/jmsc.2001.1076

Maneja RH, Frommel AY, Geffen AJ, Folkvord A, Piatkowski U, Chang MY, Clemmesen C (2013) Effects of ocean acidification on the calcification of otoliths of larval Atlantic cod Gadus morhua. Mar Ecol Prog Ser 477:251-258. doi: 10.3354/meps10146

Markow TA (1979) Phototactic behavior of Drosophila species at different temperatures. Am Nat 114:884-892.

Marshall DJ (2015) Environmentally induced (co)variance in sperm and offspring phenotypes as a source of epigenetic effects. J Exp Biol 218:107-113. doi: 10.1242/jeb.106427

Marshall DJ, Bonduriansky R, Bussière LF (2008) Offspring size variation within broods as a bet-hedging strategy in unpredictable environments. Ecology 89:2506-2517.

Martin S, Richier S, Pedrotti M-L, Dupont S, Castejon C, Gerakis Y, Kerros M-E, Oberhänsli F, Teyssié J-L, Jeffree R, Gattuso J-P (2011) Early development and molecular plasticity in the Mediterranean sea urchin Paracentrotus lividus exposed to CO2-driven acidification. J Exp Biol 214:1357-1368. doi: 10.1242/jeb.051169

Martins RS, Roberts MJ, Chang N, Verley P, Moloney CL, Vidal EAG (2010a) Effect of yolk utilization on the specific gravity of chokka squid (Loligo reynaudii) paralarvae: Implications for dispersal on the Agulhas Bank, South Africa. ICES J Mar Sci 67:13231335. doi: 10.1093/icesjms/fsq098

Martins RS, Roberts MJ, Vidal ÉAG, Moloney CL (2010b) Effects of temperature on yolk utilization by chokka squid (Loligo reynaudii d'Orbigny, 1839) paralarvae. J Exp Mar Bio Ecol 386:19-26. doi: 10.1016/j.jembe.2010.02.014

Maxwell MR, Hanlon RT (2000) Female reproductive output in the squid Loligo pealeii: Multiple egg clutches and implications for a spawning strategy. Mar Ecol Prog Ser 199:159-170. doi: 10.3354/meps199159

McCorkle DC, Weidman C, Cohen AL (2012) Time series of pCO2, pH, and aragonite saturation state in Waquoit Bay National Estuarine Research Reserve: "estaurine acidification" and shellfish. In: Ocean Sciences Meeting. Salt Lake City, UT,

McMahon JJ, Summers WC (1971) Temperature effects on the developmental rate of squid (Loligo pealei) embryos. Biol Bull 141:561-567. 
Mehrbach C, Culberson CH, Hawley JE, Pytkowicz RM (1973) Measurement of the apparent dissociation constants of carbonic acid in seawater at atmospheric pressure. Limnol Oceanogr 18:897-907. doi: 10.4319/lo.1973.18.6.0897

Mesnil B (1977) Growth and Life Cycle of Squid, Loligo pealei and Illex illecebrosus, from the Northwest Atlantic. In: Selected Papers Number 2. International Commission for the Northwest Atlantic Fisheries, Dartmouth, Canada, pp 55-69

Messenger JB (1970) Optomotor responses and nystagmus in intact, blinded and statocystless cuttlefish (Sepia officinalis L.). J Exp Biol 53:789-796.

Michaelidis B, Ouzounis C, Paleras A, Pörtner H-O (2005) Effects of long-term moderate hypercapnia on acid-base balance and growth rate in marine mussels Mytilus galloprovincialis. Mar Ecol Prog Ser 293:109-118. doi: 10.3354/meps293109

Miller GM, Watson S-A, Donelson JM, McCormick MI, Munday PL (2012) Parental environment mediates impacts of increased carbon dioxide on a coral reef fish. Nat Clim Chang 2:858-861. doi: 10.1038/nclimate1599

Moltschaniwskyj NA, Pecl GT (2007) Spawning aggregations of squid (Sepioteuthis australis) populations: a continuum of 'microcohorts.' Rev Fish Biol Fish 17:183-195. doi: 10.1007/s11160-006-9025-7

Moreno A, Dos Santos A, Piatkowski U, Santos AMP, Cabral H (2008) Distribution of cephalopod paralarvae in relation to the regional oceanography of the western Iberia. $\mathrm{J}$ Plankton Res 31:73-91. doi: 10.1093/plankt/fbn103

Moreno A, Pierce GJ, Azevedo M, Pereira J, Santos AMP (2012) The effect of temperature on growth of early life stages of the common squid Loligo vulgaris. J Mar Biol Assoc United Kingdom 92:1619-1628. doi: 10.1017/S0025315411002141

Munday PL (2014) Transgenerational acclimation of fishes to climate change and ocean acidification. F1000Prime Rep 6:1-7. doi: 10.12703/P6-99

Munday PL, Leis JM, Lough JM, Paris CB, Kingsford MJ, Berumen ML, Lambrechts J (2009a) Climate change and coral reef connectivity. Coral Reefs 28:379-395. doi: 10.1007/s00338008-0461-9

Munday PL, Donelson JM, Dixson DL, Endo GGK (2009b) Effects of ocean acidification on the early life history of a tropical marine fish. Proc R Soc B Biol Sci 276:3275-3283. doi: 10.1098/rspb.2009.0784

Munday PL, Dixson DL, Donelson JM, Jones GP, Pratchett MS, Devitsina G V, Døving KB (2009c) Ocean acidification impairs olfactory discrimination and homing ability of a marine fish. Proc Natl Acad Sci U S A 106:1848-52. doi: 10.1073/pnas.0809996106

Munday PL, Hernaman V, Dixson DL, Thorrold SR (2011) Effect of ocean acidification on otolith development in larvae of a tropical marine fish. Biogeosciences 8:1631-1641. doi: 10.5194/bg-8-1631-2011

Munday PL, Pratchett MS, Dixson DL, Donelson JM, Endo GGK, Reynolds AD, Knuckey R (2013) Elevated CO2 affects the behavior of an ecologically and economically important coral reef fish. Mar Biol 160:2137-2144. doi: 10.1007/s00227-012-2111-6

Munday PL, Cheal AJ, Dixson DL, Rummer JL, Fabricius KE (2014) Behavioural impairment in 
reef fishes caused by ocean acidification at CO 2 seeps. Nat Clim Chang 4:1-6. doi: 10.1038/NCLIMATE2195

Murray CS, Malvezzi A, Gobler CJ, Baumann H (2014) Offspring sensitivity to ocean acidification changes seasonally in a coastal marine fish. Mar Ecol Prog Ser 504:1-11. doi: 10.3354/meps10791

Nakamura M, Ohki S, Suzuki A, Sakai K (2011) Coral larvae under ocean acidification: Survival, metabolism, and metamorphosis. PLoS One 6:1-7. doi: 10.1371/journal.pone.0014521

Navarro J, Coll M, Somes CJ, Olson RJ (2013) Trophic niche of squids: Insights from isotopic data in marine systems worldwide. Deep Res Part II Top Stud Oceanogr 95:93-102. doi: 10.1016/j.dsr2.2013.01.031

Navarro MO, Bockmon EE, Frieder CA, Gonzalez JP, Levin LA (2014) Environmental pH, $\mathrm{O}_{2}$ and capsular effects on the geochemical composition of statoliths of embryonic squid Doryteuthis opalescens. Water 2233-2254. doi: 10.3390/w6082233

Navarro MO, Kwan GT, Batalov O, Choi CY, Pierce NT, Levin LA (2016) Development of embryonic market squid, Doryteuthis opalescens, under chronic exposure to low environmental pH and [O2]. PLoS One 11:e0167461. doi: 10.1371/journal.pone.0167461

Navarro MO, Parnell PE, Levin LA (2018) Essential market squid (Doryteuthis opalescens) Embryo Habitat: A Baseline for Anticipated Ocean Climate Change. J Shellfish Res 37:601-614. doi: 10.2983/035.037.0313

Nguyen HD, Doo SS, Soars NA, Byrne M (2012) Noncalcifying larvae in a changing ocean: Warming, not acidification/hypercapnia, is the dominant stressor on development of the sea star Meridiastra calcar. Glob Chang Biol 18:2466-2476. doi: 10.1111/j.13652486.2012.02714.x

Nilsson GE, Dixson DL, Domenici P, McCormick MI, Sørensen C, Watson S, Munday PL (2012) Near-future carbon dioxide levels alter fish behaviour by interfering with neurotransmitter function. Nat Clim Chang 2:201-204. doi: 10.1038/nclimate1352

NOAA (2019) Squid, Mackerel, and Butterfish Quota Monitoring Page. In: NOAA Fish. - Gt. Atl. Reg. https://www.greateratlantic.fisheries.noaa.gov/aps/monitoring/longfinsquid.html. Accessed 16 Mar 2019

Noisette F, Comtet T, Legrand E, Bordeyne F, Davoult D, Martin S (2014) Does encapsulation protect embryos from the effects of ocean acidification? The example of Crepidula fornicata. PLoS One 9:e93021. doi: 10.1371/journal.pone.0093021

O’Dor RK, Webber DM (1986) The constraints on cephalopods: why squid aren't fish. Can J Zool 64:1591-1605. doi: 10.1139/z86-241

Oyarzun FX, Strathmann RR (2011) Plasticity of hatching and the duration of planktonic development in marine invertebrates. Integr Comp Biol 51:81-90. doi: 10.1093/icb/icr009

Pachauri RK, Meyer L a. (2014) Climate Change 2014 Synthesis Report. Contribution of Working Groups I, II, and III to the Fifth Assessment Report of the Intergovernmental Panel on Climate Change.

Pansch C, Nasrolahi A, Appelhans YS, Wahl M (2012) Impacts of ocean warming and 
acidification on the larval development of the barnacle Amphibalanus improvisus. J Exp Mar Bio Ecol 420-421:48-55. doi: 10.1016/j.jembe.2012.03.023

Parker LM, Ross PM, O’Connor WA, Pörtner HO, Scanes E, Wright JM (2013) Predicting the response of molluscs to the impact of ocean acidification. Biology (Basel) 2:651-92. doi: 10.3390/biology2020651

Parmesan C (2006) Ecological and Evolutionary Responses to Recent Climate Change. Annu Rev Ecol Evol Syst 37:637-669. doi: 10.2307/annurev.ecolsys.37.091305.30000024

Pecl GT (2004) The in situ relationships between season of hatching, growth and condition in the southern calamary, Sepioteuthis australis. Mar Freshw Res 55:429-438. doi: 10.1071/MF03150

Pecl GT, Jackson GD (2008) The potential impacts of climate change on inshore squid: Biology, ecology and fisheries. Rev Fish Biol Fish 18:373-385. doi: 10.1007/s11160-007-9077-3

Pecl GT, Moltschaniwskyj NA (2006) Life history of a short-lived squid (Sepioteuthis australis): resource allocation as a function of size, growth, maturation, and hatching season. ICES J Mar Sci 63:995-1004. doi: 10.1016/j.icesjms.2006.04.007

Pecl GT, Moltschaniwskyj NA, Tracey SR, Jordan AR (2004a) Inter-annual plasticity of squid life history and population structure: Ecological and management implications. Oecologia 139:515-524. doi: 10.1007/s00442-004-1537-z

Pecl GT, Steer MA, Hodgson KE (2004b) The role of hatchling size in generating the intrinsic size-at-age variability of cephalopods: Extending the Forsythe Hypothesis. Mar Freshw Res 55:387-394. doi: 10.1071/MF03153

Perales-Raya C, Jurado-Ruzafa A, Bartolomé A, Duque V, Carrasco MN, Fraile-Nuez E (2014) Age of spent Octopus vulgaris and stress mark analysis using beaks of wild individuals. Hydrobiologia 725:105-114. doi: 10.1007/s10750-013-1602-x

Pershing AJ, Alexander MA, Hernandez CM, Kerr LA, Bris A Le, Mills KE, Nye JA, Record NR, Scannell HA, Scott JD, Sherwood GD, Thomas AC (2015) Slow adaptation in the face of rapid warming leads to collapse of the Gulf of Maine cod fishery. Science (80- ). doi: 10.1126/science.aac9819

Pierce GJ, Boyle PR (2003) Empirical modelling of interannual trends in abundance of squid (Loligo forbesi) in Scottish waters. Fish Res 59:305-326. doi: 10.1016/S01657836(02)00028-0

Pierrot D, Lewis E, Wallace DWR (2006) MS Excel program developed for CO2 system calculations. In: ORNL/CDIAC-105a. Carbon Dioxide Information Analysis Center, Oak Ridge National Laboratory, U.S. Department of Energy, Oak Ridge, Tennessee, pp 1-17

Pimentel M, Pegado M, Repolho T, Rosa R (2014a) Impact of ocean acidification in the metabolism and swimming behavior of the dolphinfish (Coryphaena hippurus) early larvae. Mar Biol 161:725-729. doi: 10.1007/s00227-013-2365-7

Pimentel MS, Trübenbach K, Faleiro F, Boavida-Portugal J, Repolho T, Rosa R, Trubenbach K, Faleiro F, Boavida-Portugal J, Repolho T, Rosa R (2012) Impact of ocean warming on the early ontogeny of cephalopods: A metabolic approach. Mar Biol 159:2051-2059. doi:

10.1007/s00227-012-1991-9 
Pimentel MS, Faleiro F, Dionísio G, Repolho T, Pousão-Ferreira P, Machado J, Rosa R (2014b) Defective skeletogenesis and oversized otoliths in fish early stages in a changing ocean. $\mathrm{J}$ Exp Biol 217:2062-70. doi: 10.1242/jeb.092635

Pörtner H-O (1990) An analysis of the effects of $\mathrm{pH}$ on oxygen binding by squid (Illex illecebrosus, Loligo pealei) hemocyanin. J Exp Biol 424:407-424.

Pörtner H-O, Webber DM, Boutilier RG, O’Dor RK (1991) Acid-Base Regulation in Exercising Squid. Am. J. Physiol. 261:R239-R246.

Pörtner H-O, Reipschlager A, Heisler N (1998) Acid-base regulation, metabolism and energetics in Sipunculus nudus as a function of ambient carbon dioxide level. J Exp Biol 201:43-55.

Pörtner HO (1994) Coordination of metabolism, acid-base regulation and haemocyanin function in cephalopods. Mar Freshw Behav Physiol 25:131-148.

Putnam HM, Gates RD (2015) Preconditioning in the reef-building coral Pocillopora damicornis and the potential for trans-generational acclimatization in coral larvae under future climate change conditions. J Exp Biol 218:2365-2372. doi: 10.1242/jeb.123018

Radtke RL (1983) Chemical and structural characteristics of statoliths from the short-finned squid Illex illecebrosus. Mar Biol 76:47-54. doi: 10.1007/BF00393054

Redfield AC, Goodkind R (1929) The significance of the Bohr Effect in the respiration and asphyxiation of the squid, Loligo pealei. J Exp Biol 6:340-349.

Réveillac E, Lacoue-Labarthe T, Oberhänsli F, Teyssié J-L, Jeffree R, Gattuso J-P, Martin S (2015) Ocean acidification reshapes the otolith-body allometry of growth in juvenile sea bream. J Exp Mar Bio Ecol 463:87-94. doi: 10.1016/j.jembe.2014.11.007

Ries JB, Cohen AL, McCorkle DC (2009) Marine calcifiers exhibit mixed responses to CO2induced ocean acidification. Geology 37:1131-1134. doi: 10.1130/G30210A.1

Rivest EB, Hofmann GE (2014) Responses of the metabolism of the larvae of Pocillopora damicornis to ocean acidification and warming. PLoS One 9:e96172. doi: 10.1371/journal.pone.0096172

Roberts MJ (2005) Chokka squid (Loligo vulgaris reynaudii) abundance linked to changes in South Africa's Agulhas Bank ecosystem during spawning and the early life cycle. ICES J Mar Sci 62:33-55. doi: 10.1016/j.icesjms.2004.10.002

Robin JP, Roberts M, Zeidberg L, Bloor I, Rodriguez A, Briceño F, Downey N, Mascaró M, Navarro M, Guerra A, Hofmeister J, Barcellos DD, Lourenço SAP, Roper CFE, Moltschaniwskyj NA, Green CP, Mather J (2014) Transitions during cephalopod life history: The role of habitat, environment, functional morphology and behaviour. In: Vidal EAG (ed) Advances in Cephalopod Science: Biology, Ecology, Cultivation and Fisheries. Academic Press, Cambridge, MA, pp 361-437

Rodhouse PGK, Pierce GJ, Nichols OC, Sauer WHH, Arkhipkin AI, Laptikhovsky V V., Lipiński MR, Ramos JE, Gras M, Kidokoro H, Sadayasu K, Pereira J, Lefkaditou E, Pita C, Gasalla M, Haimovici M, Sakai M, Downey N (2014) Environmental effects on cephalopod population dynamics: Implications for management of fisheries. Adv Mar Biol 67:99-233. doi: 10.1016/B978-0-12-800287-2.00002-0

Rodolfo-Metalpa R, Houlbrèque F, Tambutté É, Boisson F, Baggini C, Patti FP, Jeffree R, Fine 
M, Foggo A, Gattuso J-P, Hall-Spencer JM (2011) Coral and mollusc resistance to ocean acidification adversely affected by warming. Nat Clim Chang 1:308-312. doi:

10.1038/nclimate1200

Rosa R, Seibel BA (2008) Synergistic effects of climate-related variables suggest future physiological impairment in a top oceanic predator. Proc Natl Acad Sci 105:20776-20780. doi: 10.1073/pnas.0806886105

Rosa R, Seibel BA (2010) Metabolic physiology of the Humboldt squid, Dosidicus gigas: Implications for vertical migration in a pronounced oxygen minimum zone. Prog Oceanogr 86:72-80. doi: 10.1016/j.pocean.2010.04.004

Rosa R, Pimentel MS, Boavida-Portugal J, Teixeira T, Trübenbach K, Diniz M (2012) Ocean warming enhances malformations, premature hatching, metabolic suppression and oxidative stress in the early life stages of a keystone squid. PLoS One. doi:

10.1371/journal.pone.0038282

Rosa R, Trübenbach K, Repolho T, Pimentel M, Faleiro F, Boavida-Portugal J, Baptista M, Lopes VM, Dionísio G, Leal MC, Calado R, Pörtner HO (2013) Lower hypoxia thresholds of cuttlefish early life stages living in a warm acidified ocean. Proc R Soc B Biol Sci 280:20131695. doi: 10.1098/rspb.2013.1695

Rosa R, Trübenbach K, Pimentel MS, Boavida-Portugal J, Faleiro F, Baptista M, Dionísio G, Calado R, Pörtner HO, Repolho T (2014a) Differential impacts of ocean acidification and warming on winter and summer progeny of a coastal squid (Loligo vulgaris). J Exp Biol 217:518-25. doi: 10.1242/jeb.096081

Rosa R, O’Dor R, Pierce G (2014b) Myopsid Squids. Nova Science Publishers, Inc, New York, NY

Rosenthal JJC (2015) The emerging role of RNA editing in plasticity. J Exp Biol 218:18121821. doi: 10.1242/jeb.119065

Ross PM, Parker L, O’Connor W a., Bailey E a. (2011) The Impact of Ocean Acidification on Reproduction, Early Development and Settlement of Marine Organisms. Water 3:10051030. doi: 10.3390/w3041005

Ross PM, Parker L, Byrne M (2012) Transgenerational responses of molluscs and echinoderms to changing ocean conditions. 69:380-388. doi: 10.1093/icesjms/fst048

Ruby EG, McFall-Ngai MJ (1992) A squid that glows in the night: Development of an animalbacterial mutualism. J Bacteriol 174:4865-4870.

Saba VS, Griffies SM, Anderson WG, Winton M, Alexander MA, Delworth TL, Hare JA, Harrison MJ, Rosati A, Vecchi GA, Zhang R (2016) Enhanced warming of the Northwest Atlantic Ocean under climate change. J Geophys Res Ocean 121:118-132. doi: 10.1002/2015JC011346

Sabine CL, Feely R a, Gruber N, Key RM, Lee K, Bullister JL, Wanninkhof R, Wong CS, Wallace DWR, Tilbrook B, Millero FJ, Peng T-H, Kozyr A, Ono T, Rios AF (2004) The oceanic sink for anthropogenic CO2. Science 305:367-371. doi: 10.1126/science. 1097403

Schunter C, Welch MJ, Ryu T, Zhang H, Berumen ML, Nilsson GE, Munday PL, Ravasi T (2016) Molecular signatures of transgenerational response to ocean acidification in a species of reef fish. Nat Clim Chang 6:1014-1018. doi: 10.1038/nclimate3087 
Schunter C, Welch MJ, Nilsson GE, Rummer JL, Munday PL, Ravasi T (2018) An interplay between plasticity and parental phenotype determines impacts of ocean acidification on a reef fish. Nat Ecol Evol 2:334-342. doi: 10.1038/s41559-017-0428-8

Seibel BA (2013) The jumbo squid, Dosidicus gigas (Ommastrephidae), living in oxygen minimum zones II: Blood-oxygen binding. Deep Res Part II Top Stud Oceanogr 95:139144. doi: $10.1016 /$ j.dsr2.2012.10.003

Seibel BA (2015) Environmental physiology of the jumbo squid, Dosidicus gigas (d 'Orbigny, 1835) (Cephalopoda : Ommastrephidae): Implications for changing climate. Am Malacol Bull 33:1-13.

Seibel BA (2016) Cephalopod susceptibility to asphyxiation via ocean incalescence, deoxygenation, and acidification. Physiology 31:418-429. doi: 10.1152/physiol.00061.2015

Seibel BA, Hochberg FG, Carlini DB (2000) Life history of Gonatus onyx (Cephalopoda: Teuthoidea): deep-sea spawning and post-spawning egg care. Mar Biol 137:519-526. doi: $10.1007 / \mathrm{s} 002270000359$

Seibel BA, Maas AE, Dierssen HM (2012) Energetic plasticity underlies a variable response to ocean acidification in the pteropod, Limacina helicina antarctica. PLoS One 7:e30464. doi: 10.1371/journal.pone.0030464

Seibel BA, Hafker NS, Trubenbach K, Zhang J, Tessier SN, Portner H-O, Rosa R, Storey KB (2014) Metabolic suppression during protracted exposure to hypoxia in the jumbo squid, Dosidicus gigas, living in an oxygen minimum zone. J Exp Biol 217:2555-2568. doi: 10.1242/jeb.100487

Semmens JM, Pecl GT, Gillanders BM, Waluda CM, Shea EK, Jouffre D, Ichii T, Zumholz K, Katugin ON, Leporati SC, Shaw PW (2007) Approaches to resolving cephalopod movement and migration patterns. Rev Fish Biol Fish 17:401-423. doi: 10.1007/s11160007-9048-8

Sen H (2005) Temperature tolerance of loliginid squid (Loligo vulgaris Lamarck, 1798) eggs in controlled conditions. Turkish J Fish Aquat Sci 5:53-56.

Shadwick RE, O’Dor RK, Gosline JM (1990) Respiratory and cardiac function during exercise in squid. Can J Zool 68:792-798. doi: 10.1139/z90-114

Shashar N, Hanlon RT (2013) Spawning behavior dynamics at communal egg beds in the squid Doryteuthis (Loligo) pealeii. J Exp Mar Bio Ecol 447:65-74. doi: 10.1016/j.jembe.2013.02.011

Shaw EC, Munday PL, McNeil BI (2013) The role of $\mathrm{CO}_{2}$ variability and exposure time for biological impacts of ocean acidification. Geophys Res Lett 40:4685-4688. doi: $10.1002 /$ grl.50883

Shea EK, Vecchione M (2010) Ontogenic changes in diel vertical migration patterns compared with known allometric changes in three mesopelagic squid species suggest an expanded definition of a paralarva. ICES J Mar Sci 67:1436-1443. doi: 10.1093/icesjms/fsq104

Sigwart JD, Lyons G, Fink A, Gutowska M a, Murray D, Melzner F, Houghton JDR, Hu MY (2016) Elevated $\mathrm{pCO}_{2}$ drives lower growth and yet increased calcification in the early life history of the cuttlefish Sepia officinalis. ICES J Mar Sci 73:970-980. doi: 10.1093/icesjms/fst176 
Smith AM, Wolfe K, Byrne M (2012) Argonauta at risk: dissolution and carbonate mineralogy of egg cases. 1:2010-2013.

Sokolova IM (2013) Energy-limited tolerance to stress as a conceptual framework to integrate the effects of multiple stressors. Integr Comp Biol 53:597-608. doi: 10.1093/icb/ict028

Sokolova IM, Frederich M, Bagwe R, Lannig G, Sukhotin AA (2012) Energy homeostasis as an integrative tool for assessing limits of environmental stress tolerance in aquatic invertebrates. Mar Environ Res 79:1-15. doi: 10.1016/j.marenvres.2012.04.003

Spady BL, Watson S, Chase TJ, Munday PL (2014) Projected near-future $\mathrm{CO}_{2}$ levels increase activity and alter defensive behaviours in the tropical squid Idiosepius pygmaeus. Biol Open 3:1063-70. doi: 10.1242/bio.20149894

Spady BL, Nay TJ, Rummer JL, Munday PL, Watson S-A (2019) Aerobic performance of two tropical cephalopod species unaltered by prolonged exposure to projected future carbon dioxide levels. Conserv Physiol 7:1-11. doi: 10.1093/conphys/coz024

Staaf DJ, Zeidberg LD, Gilly WF (2011) Effects of temperature on embryonic development of the Humboldt squid Dosidicus gigas. Mar Ecol Prog Ser 441:165-175. doi: 10.3354/meps09389

Staaf DJ, Gilly WF, Denny MW (2014) Aperture effects in squid jet propulsion. J Exp Biol 217:1588-600. doi: $10.1242 /$ jeb.082271

Stamhuis E, Videler J (1995) Quantitative flow analysis around aquatic animals using laser sheet particle image velocimetry. J Exp Biol 198:283-94.

Steer M, Moltschaniwskyj N, Nichols D, Miller M (2004) The role of temperature and maternal ration in embryo survival: Using the dumpling squid Euprymna tasmanica as a model. J Exp Mar Bio Ecol 307:73-89. doi: 10.1016/j.jembe.2004.01.017

Steer MA, Moltschaniwskyj NA (2007) The effects of egg position, egg mass size, substrate and biofouling on embryo mortality in the squid Sepioteuthis australis. Rev Fish Biol Fish 17:173-182. doi: 10.1007/s11160-006-9023-9

Steer MA, Pecl GT, Moltschaniwskyj NA (2003) Are bigger calamary Sepioteuthis australis hatchlings more likely to survive? A study based on statolith dimensions. Mar Ecol Prog Ser 261:175-182. doi: 10.3354/meps261175

Strobel A, Hu MYA, Gutowska MA, Lieb B, Lucassen M, Melzner F, Pörtner HO, Mark FC (2012) Influence of Temperature, Hypercapnia, and Development on the Relative Expression of Different Hemocyanin Isoforms in the Common Cuttlefish Sepia officinalis. J Exp Zool Part A Ecol Genet Physiol 317:511-523. doi: 10.1002/jez.1743

Stumpp M, Dupont S, Thorndyke MC, Melzner F (2011) CO2 induced seawater acidification impacts sea urchin larval development II: Gene expression patterns in pluteus larvae. Comp Biochem Physiol Part A Mol Integr Physiol 160:320-330. doi: 10.1016/j.cbpa.2011.06.023

Summers WC (1971) Age and growth of Loligo pealei, a population study of the common Atlantic coast squid. Biol Bull 141:189-201.

Summers WC, McMahon JJ, Ruppert GNPA (1974) Studies on the maintenance of adult squid (Loligo peali). II. Empirical Extensions. Biol Bull 146:291-301.

Sunday JM, Calosi P, Dupont S, Munday PL, Stillman JH, Reusch TBH (2014) Evolution in an 
acidifying ocean. Trends Ecol Evol 29:117-125. doi: 10.1016/j.tree.2013.11.001

Takesue RK, Bacon CR, Thompson JK (2008) Influences of organic matter and calcification rate on trace elements in aragonitic estuarine bivalve shells. Geochim Cosmochim Acta 72:5431-5445. doi: 10.1016/j.gca.2008.09.003

Tambutté E, Venn AA, Holcomb M, Segonds N, Techer N, Zoccola D, Allemand D, Tambutté S (2015) Morphological plasticity of the coral skeleton under CO2-driven seawater acidification. Nat Commun 6:7368. doi: 10.1038/ncomms8368

Thompson JT, Bartol IK, Baksi AE, Li KY, Krueger PS (2010) The ontogeny of muscle structure and locomotory function in the long-finned squid Doryteuthis pealeii. J Exp Biol 213:1079-1091. doi: 10.1242/jeb.034553

Tian Y (2009) Interannual-interdecadal variations of spear squid Loligo bleekeri abundance in the southwestern Japan Sea during 1975-2006: Impact of the trawl fishing and recommendations for management under the different climate regimes. Fish Res 100:78-85. doi: 10.1016/j.fishres.2009.06.005

Trueblood LA, Seibel BA (2013) The jumbo squid, Dosidicus gigas (Ommastrephidae), living in oxygen minimum zones I: Oxygen consumption rates and critical oxygen partial pressures. Deep Res Part II Top Stud Oceanogr 95:218-224. doi: 10.1016/j.dsr2.2012.10.004

van der Sman J, Phillips NE, Pfister CA (2009) Relative effects of maternal and juvenile food availability for a marine snail. Ecology 90:3119-3125.

Vargas CA, Lagos NA, Lardies MA, Duarte C, Manríquez PH, Aguilera VM, Broitman B, Widdicombe S, Dupont S (2017) Species-specific responses to ocean acidification should account for local adaptation and adaptive plasticity. Nat Ecol Evol 1:1-7. doi: 10.1038/s41559-017-0084

Vecchione M (1981) Aspects of the early life history of Loligo pealeii (Cephalopoda; Myopsida). J Shellfish Res 1:171-180.

Vecchione M, Roper CFE, Sweeney MJ, Lu CC (2001) Distribution, relative abundance and developmental morphology of paralarval cephalopods in the western North Atlantic Ocean. In: NOAA Technical Report NMFS 152.

Vézina A, Hoegh-Guldberg O (2008) Effects of ocean acidification on marine ecosystems. Mar Ecol Prog Ser 373:199-201. doi: 10.3354/meps07868

Vidal EAG, Haimovici M (1998) Feeding and the possible role of the proboscis and mucus cover in the ingestion of microorganism by rhynchoteuthion paralarvae (Cephalopoda: Ommastrephidae). Bull Mar Sci 63:305-316.

Vidal EAG, DiMarco FP, Wormuth JH, Lee PG (2002a) Influence of temperature and food availability on survival, growth and yolk utilization in hatchling squid. Bull Mar Sci 71:915-931.

Vidal EAG, DiMarco FP, Wormuth JH, Lee PG (2002b) Optimizing rearing conditions of hatchling loliginid squid. Mar Biol 140:117-127. doi: 10.1007/s002270100683

Vidal EAG, Villanueva R, Andrade JP, Gleadall IG, Iglesias J, Koueta N, Rosas C, Segawa S, Grasse B, Franco-Santos RM, Albertin CB, Caamal-Monsreal C, Chimal ME, EdsingerGonzales E, Gallardo P, Le Pabic C, Pascual C, Roumbedakis K, Wood J (2014) 
Cephalopod culture: Current status of main biological models and research priorities. Adv Mar Biol 67:1-98. doi: 10.1016/B978-0-12-800287-2.00001-9

Villanueva R, Nozais CC, Boletzky S v. (1997) Swimming behaviour and food searching in planktonic Octopus vulgaris Cuvier from hatching to settlement. J Exp Mar Bio Ecol 208:169-184. doi: 10.1016/S0022-0981(96)02670-6

Villanueva R, Arkhipkin A, Jereb P, Lefkaditou E, Lipinski MR, Perales-Raya C, Riba J, Rocha F (2003) Embryonic life of the loliginid squid Loligo vulgaris: Comparison between statoliths of Atlantic and Mediterranean populations. Mar Ecol Prog Ser 253:197-208. doi: $10.3354 /$ meps 253197

Villanueva R, Quintana D, Petroni G, Bozzano A (2011) Factors influencing the embryonic development and hatchling size of the oceanic squid Illex coindetii following in vitro fertilization. J Exp Mar Bio Ecol 407:54-62. doi: 10.1016/j.jembe.2011.07.012

Vogel S (1981) Life in Moving Fluids: The Physical Biology of Flow, 2nd Editio. Princeton University Press, Princeton, New Jersey

Wang ZA, Wanninkhof R, Cai W-J, Byrne RH, Hu X, Peng T-H, Huang W-J (2013) The marine inorganic carbon system along the Gulf of Mexico and Atlantic coasts of the United States : Insights from a transregional coastal carbon study. Limnol Oceanogr 58:325-342. doi: 10.4319/1o.2013.58.1.0325

Wannamaker CM, Rice JA (2000) Effects of hypoxia on movements and behavior of selected estuarine organisms from the southeastern United States. J Exp Mar Bio Ecol 249:145-163. doi: 10.1016/S0022-0981(00)00160-X

Wassersug R, von Seckendorf Hoff K (1985) The kinematics of swimming in anuran larvae. J Exp Biol 119:1-30.

Webber DM, O'Dor RK (1986) Monitoring the metabolic rate and activity of free-swimming squid with telemetered jet pressure. J Exp Biol 224:205-224.

Webber DM, Aitken JP, O'Dor RK (2010) Costs of locomotion and vertic dynamics of cephalopods and fish. Physiol Biochem Zool 73:651-62. doi: 10.1086/318100

Wheeler JD, Helfrich KR, Anderson EJ, McGann B, Staats P, Wargula AE, Wilt K, Mullineaux LS (2013) Upward swimming of competent oyster larvae Crassostrea virginica persists in highly turbulent flow as detected by PIV flow subtraction. Mar Ecol Prog Ser 488:171-185. doi: $10.3354 /$ meps 10382

Wheeler JD, Helfrich KR, Anderson EJ, Mullineaux LS (2015) Isolating the hydrodynamic triggers of the dive response in eastern oyster larvae. Limnol Oceanogr. doi: 10.1002/lno.10098

White MM, McCorkle DC, Mullineaux LS, Cohen AL (2013) Early exposure of bay scallops (Argopecten irradians) to high $\mathrm{CO}_{2}$ causes a decrease in larval shell growth. PLoS One 8:29. doi: 10.1371/journal.pone.0061065

Winans AK, Purcell JE (2010) Effects of $\mathrm{pH}$ on asexual reproduction and statolith formation of the scyphozoan, Aurelia labiata. Hydrobiologia 645:39-52. doi: 10.1007/s10750-010-02249

Xavier JC, Allcock AL, Cherel Y, Lipinski MR, Pierce GJ, Rodhouse PGK, Rosa R, Shea EK, 
Strugnell JM, Vidal EAG, Villanueva R, Ziegler A (2015) Future challenges in cephalopod research. J Mar Biol Assoc United Kingdom 95:999-1015. doi:

$10.1017 / \mathrm{S} 0025315414000782$

Yamaoka K, Nanbu T, Miyagawa M, Isshiki T, Kusaka a. (2000) Water surface tension-related deaths in prelarval red-spotted grouper. Aquaculture 189:165-176. doi: 10.1016/S00448486(00)00354-9

Yatsu A, Watanabe T, Mori J, Nagasawa K, Ishida Y, Meguro T, Kamei Y, Sakurai Y (2000) Interannual variability in stock abundance of the neon flying squid, Ommastrephes bartramii, in the North Pacific Ocean during 1979-1998: impact of driftnet fishing and oceanographic conditions. Fish Oceanogr 9:163-170.

York CA, Bartol IK (2016) Anti-predator behavior of squid throughout ontogeny. J Exp Mar Bio Ecol 480:26-35. doi: 10.1016/j.jembe.2016.03.011

York CA, Bartol IK, Krueger PS (2016) Multiple sensory modalities used by squid in successful predator evasion throughout ontogeny. J Exp Biol jeb.140780. doi: 10.1242/jeb.140780

Zakroff C, Mooney TA, Wirth C (2018) Ocean acidification responses in paralarval squid swimming behavior using a novel 3D tracking system. Hydrobiologia 808:83-106. doi: 10.1007/s10750-017-3342-9

Zakroff C, Mooney TA, Berumen ML (2019) Dose-dependence and small-scale variability in responses to ocean acidification during squid, Doryteuthis pealeii, development. Mar Biol 166:62. doi: 10.1007/s00227-019-3510-8

Zakroff CJ (2013) CO2-level dependent effects of ocean acidification on squid, Doryteuthis pealeii, early life history. Master's thesis. King Abdullah University of Science and Technology

Zeidberg LD, Isaac G, Widmer CL, Neumeister H, Gilly WF (2011) Egg capsule hatch rate and incubation duration of the California market squid, Doryteuthis (=Loligo) opalescens: Insights from laboratory manipulations. Mar Ecol 32:468-479. doi: 10.1111/j.14390485.2011.00445.x

Zielinski S, Sartoris FJ, Portner HO (2001) Temperature effects on hemocyanin oxygen binding in an Antartic cephalopod. Biol Bull 200:67-76. 